\author{
This electronic thesis or dissertation has been \\ downloaded from the King's Research Portal at \\ https://kclpure.kcl.ac.uk/portal/
}

\title{
Optimising Physiology Guided Management of Serial Coronary Artery Disease
}

\author{
Modi, Bhavik \\ Awarding institution: \\ King's College London
}

The copyright of this thesis rests with the author and no quotation from it or information derived from it may be published without proper acknowledgement.

\section{END USER LICENCE AGREEMENT}

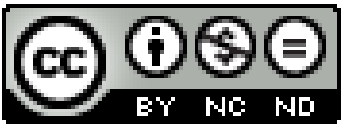

Unless another licence is stated on the immediately following page this work is licensed

under a Creative Commons Attribution-NonCommercial-NoDerivatives 4.0 International

licence. https://creativecommons.org/licenses/by-nc-nd/4.0/

You are free to copy, distribute and transmit the work

Under the following conditions:

- $\quad$ Attribution: You must attribute the work in the manner specified by the author (but not in any way that suggests that they endorse you or your use of the work).

- $\quad$ Non Commercial: You may not use this work for commercial purposes.

- $\quad$ No Derivative Works - You may not alter, transform, or build upon this work.

Any of these conditions can be waived if you receive permission from the author. Your fair dealings and other rights are in no way affected by the above.

Take down policy

If you believe that this document breaches copyright please contact librarypure@kcl.ac.uk providing details, and we will remove access to the work immediately and investigate your claim. 


\title{
Optimising Physiology-Guided Management of Serial Coronary Artery Disease
}

\author{
Dr Bhavik N Modi \\ MA MBBS MRCP FHEA
}

SUBMITTED FOR THE DEGREE OF DOCTOR OF PHILOSOPHY

\author{
Cardiovascular Division \\ King's College London \\ BHF Centre of Research Excellence \\ The Rayne Institute \\ St Thomas' Hospital \\ London, SE1 7EH \\ Supervisors: \\ Prof Divaka Perera \\ Prof Phil Chowienczyk
}




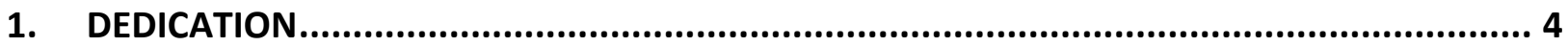

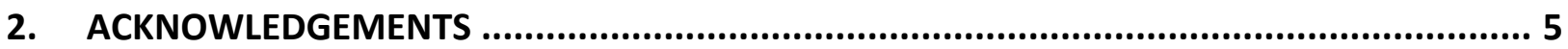

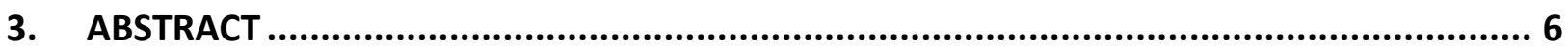

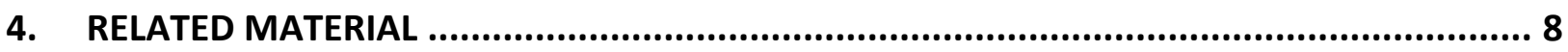

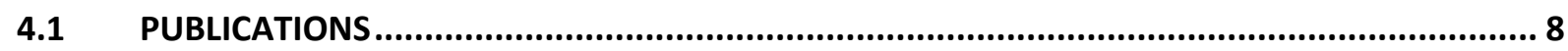

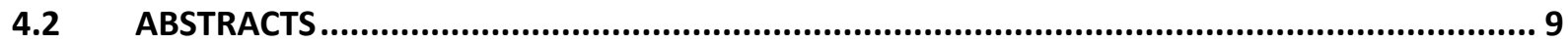

4.3 AWARDS ................................................................................................... 10

5. BACKGROUND........................................................................................................11

5.1 ASSESSING STENOSIS SEVERITY DURING ANGIOGRAPHY .............................................. 13

5.1.1 Fractional Flow Reserve, FFR............................................................................. 14

5.1.2 Resting Indices of Stenosis Severity: Resting PdPa \& iFR …………………………….... 14

5.1.3 Pressure-Flow indices of Stenosis Severity: CFR \& HSR …………................................. 15

5.2 THE PROBLEM OF SERIAL CORONARY ARTERY DISEASE.................................................. 16

5.2.1 Factors Influencing The Pressure Drop Across A Stenosis............................................... 16

5.2.2 The unique physiology of serial stenoses..................................................................... 18

5.2.3 Theoretical solutions to overcome the FFR interplay of serial stenoses........................... 19

5.3 CURRENT METHODS IN PHYSIOLOGICAL MANAGEMENT OF SERIAL AND DIFFUSE

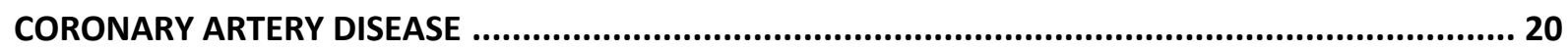

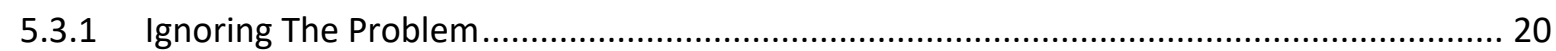

5.3.2 The 'Educated Guess' ........................................................................................ 21

5.3.3 FFR Pullback Manouvre............................................................................................ 21

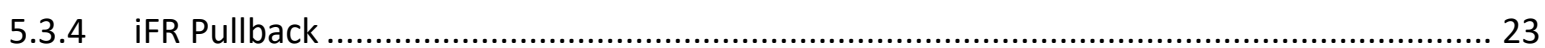

5.4 PHYSIOLOGY GUIDED MANAGEMENT OF LEFT MAIN CORONARY ARTERY DISEASE: AN

IMPORTANT EXAMPLE OF SERIAL STENOSIS INTERPLAY ........................................................ 24

5.4.1 Evidence for FFR Assessment of the LMCA ................................................................ 24

5.4.2 Theoretical Challenges to Physiological Assessment of the LMCA ……………………... 26

5.4.3 Best Practice in Left Main Physiological Recordings ....................................................... 28

5.4.4 Imaging LMCA disease as a surrogate of physiology? .................................................... 30

5.4.5 Algorithm For Physiological Assessment of LMCA Disease Incorporating the Disease Free

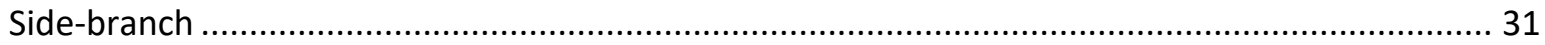

5.5 NON-INVASIVE FFR BY COMPUTED TOMOGRAPHY (FFR CT $)$................................................. 32

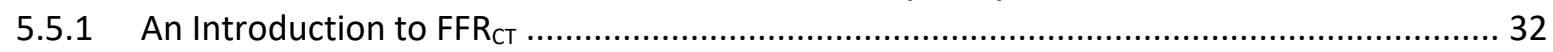

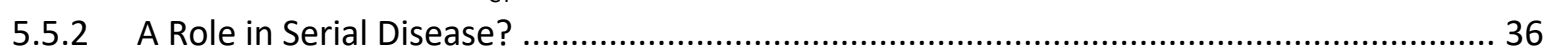

5.6 AIMS \& OBJECTIVES..................................................................................................... 37

6. EXPERIMENTAL CHAPTER: IN VITRO ASSESSMENT OF SERIAL CORONARY ARTERY

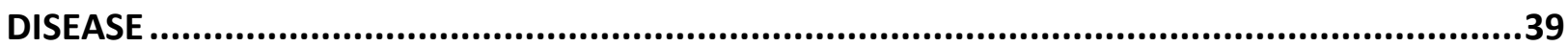

7. EXPERIMENTAL CHAPTER: PHYSIOLOGICAL CHARACTERIZATION OF SERIAL CORONARY ARTERY DISEASE: A COMPARISON OF CURRNETLY AVAILABLE PRESSURE AND RESISTANCEBASED INDICES AND A NOVEL MATHEMATICAL SOLUTION ....................................................58

8. EXPERIMENTAL CHAPTER: NON-INVASIVE COMPUTED TOMOGRAPHY (CT) IN SERIAL CORONARY ARTERY DISEASE

8.1 Predicting the Physiological Effect of Revascularisation in Serially Diseased Coronary

Arteries: Clinical Validation of a Novel CT Coronary Angiography-Based Technique ...................... 79

8.2 Estimating Hyperaemic Stenosis Resistance by Cardiac CT ........................................... 93

9. EXPERIMENTAL CHAPTER: CLINICAL UTILITY STUDY ……............................................100 


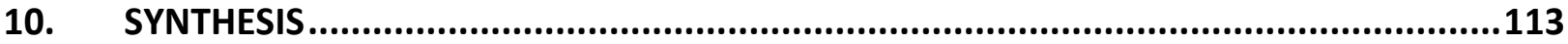

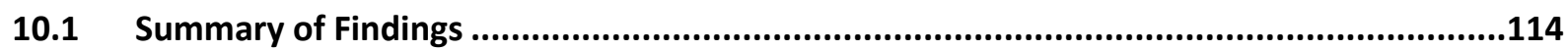

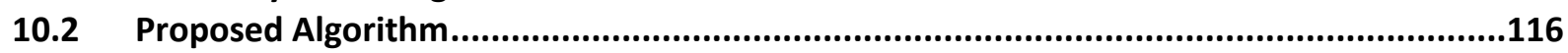

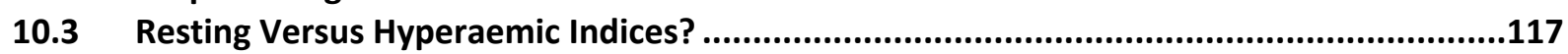

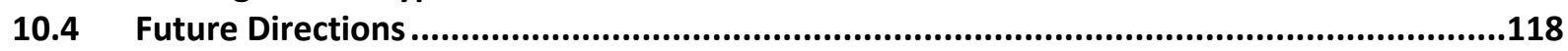

10.5 Limitations of No ‘Gold Standard' For Invasive Physiological Assessment .......................121

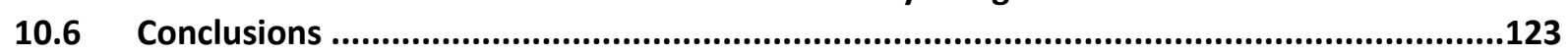

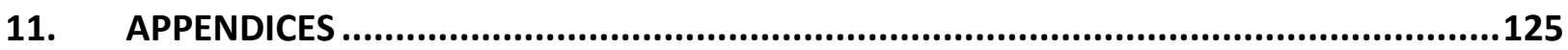

APPENDIX A: REFINING THE ASSESSMENT OF STENOSIS SEVERITY IN CLINICAL PRACTICE......126 APPENDIX B: ETHICS APPROVAL, CONSENT FORM AND PATIENT INFORMATION SHEETS ......158

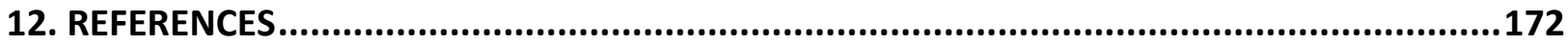




\section{DEDICATION}

First and foremost, I would like to dedicate this thesis to my wife Anika for her unwavering love, support, patience and positivity.

And then of course I dedicate this work to my parents: without you none of this would have been possible. I am forever grateful for your ongoing love, guidance and encouragement at every stage of my life, education and career. I hope I can do the same for my children. 


\section{ACKNOWLEDGEMENTS}

Over the last 3 years, my time at The Rayne Institute, St Thomas' Hospital, has given me much enjoyment and enabled me to mature as a researcher, clinician and as a person. I owe several people a great deal of thanks but a few people deserve special mention.

Professor Divaka Perera has not only been a mentor and brilliant supervisor, but an inspiration. Whilst working closely with him, I have grown to admire his enthusiasm, skill, drive and clarity of thought; whilst still being incredibly supportive, kind, thoughtful and thoroughly good company. My most enjoyable moments of these last few years have come during the numerous formal supervisions and impromptu 'catch-ups' with him. These moments have been key to the success of this work and have provided me with a great deal of guidance and motivation. I am privileged to have worked closely with him and I have no doubt he will continue to be hugely successful. I hope to continue working with him and maintaining our friendship.

I would also like to thank Professor Chowienczyk, Dr Rajani and Professor Marber. On countless occasions, just a few minutes of talking to Professor Chowienczyk and Professor Marber about a problem would yield a simple yet elegant solution: their brilliance I will always admire. When it came to the imaging branch of this work - Dr Rajani's knowledge, enthusiasm and support was incredible. Thank you to the three of you for all your expertise and guidance.

I am also grateful to the other fellows with whom I have spent these last 3 years. Thank you for the support, intellectual discussion and the good times. In particular I want to thank our research physiologist, Howie, who supports us all in acquiring and analysing physiological data.

Lastly, thank you to the patients who took part. Enrolling in research when you are undoubtedly worried about your own health is not easy and I am truly indebted to you all. 


\section{ABSTRACT}

\section{Introduction:}

Physiology-guided coronary revascularisation is associated with improved outcomes however it is unclear if physiological indices can reliably assess individual lesions in the commonlyencountered scenario of serial/diffuse coronary artery disease.

\section{Methods:}

3-D printed serial disease phantoms were assessed within an in vitro model of circulation. FFR of a lesion was predicted from the size of step-up on pressure-wire pullback in the presence of serial lesions $\left(\mathrm{FFR}_{\mathrm{app}}\right)$ and compared to phantoms with no accompanying lesion (FFR $\mathrm{Frue}_{\text {tre }}$. A mathematical model to minimise error in predicting FFR $_{\text {true }}\left(\mathrm{FFR}_{\text {pred }}\right)$ was developed in 32 phantoms and validated in another 20 phantoms. In 54 patients with serial disease, resting and hyperaemic pressure-wire pullbacks were performed, with FFR, iFR, Pd/Pa of each stenosis

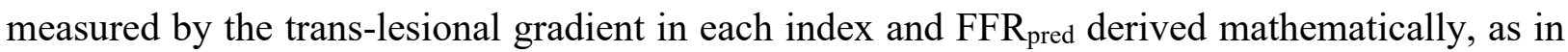
the in vitro study. These indices were compared to the 'true' trans-lesional gradient of each index following stenosis isolation by PCI of one of the stenoses or using a disease-free sidebranch. In a subset of 27 patients, Doppler average peak flow velocity (APV) was also measured before and after stenosis isolation, to calculate $\mathrm{hSR}(\mathrm{hSR}=\Delta \mathrm{P} / \mathrm{APV}$, where $\Delta \mathrm{P}=$ trans-lesional pressure gradient). In a further subset of 24 patients, prospective CT coronary angiography was performed prior to stenosis isolation by PCI to assess the value of a novel non-invasive $\mathrm{FFR}_{\mathrm{CT}}$-derived PCI

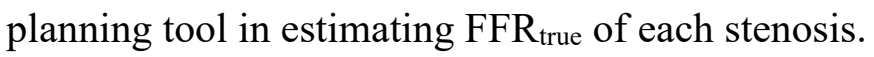

\section{Results:}

Both in vitro and in the clinical cohort, individual stenoses are underestimated and misclassified in serial disease, proportional to total FFR. Mean errors for FFR, iFR and resting $\mathrm{Pd} / \mathrm{Pa}$ were 
$33.3 \%, 19.8 \%$ and $23.4 \%$ respectively, and $14.3 \%$ for $\mathrm{FFR}_{\text {pred }}(\mathrm{P}<0.001)$. Stenosis misclassification rates based on $0.80,0.89$ and 0.91 thresholds were $17 \%, 24 \%$ and $20 \%$ for FFR, iFR and Pd/Pa respectively. With FFR pred only $11 \%$ of stenoses were misclassified. Apparent and true hSR correlated strongly $(\mathrm{R}=0.87, \mathrm{P}<0.001)$, with $7 \%$ of stenoses misclassified, thus suggesting that serial stenoses largely behave as resistors in series (providing further validation of the principles upon which our mathematical solution was based). In the subset of patients that had a prospective CT scan, applying a novel interactive PCI planning tool to $\mathrm{FFR}_{\mathrm{CT}}$ outputs results in significant improvements in the estimation of stenosis severity with the absolute value of underestimation falling to $0.01+/-0.05$ ( $7 \%$ error).

\section{Conclusion:}

Physiological assessment of stenoses in the presence of serial CAD is prone to significant underestimation, proportional to the cumulative burden of disease within the vessel. All invasive pressure-derived physiological indices, resting and hyperaemic, are prone to significant error and stenosis misclassification when used conventionally. Doppler-based resistance indices are less prone to this error but utilisation is limited by difficulties in obtaining Doppler traces. The finding that serial stenoses generally behave as resistors in series supports the use of an FFR correction equation, derived using our 3D-printed in vitro study, that does not require measurement of coronary occlusive pressure. Applying this correction equation to routine pressure wire pullback measurements significantly reduces error in estimating true stenosis significance and has significant clinical utility. In addition, we demonstrate and validate a noninvasive FFR $_{\mathrm{CT}}$-derived PCI planning tool that aids more accurate prediction of true stenosis significance in serial CAD compared to contemporary invasive and non-invasive methods. The next step is to establish multi-centre clinical utility and outcome studies of the improved physiological methods established in this thesis. 


\section{RELATED MATERIAL}

\section{Relevant to degree period Oct 2015 - Sep 2018}

\subsection{PUBLICATIONS}

1. Rajani R, Modi B, Ntalas I, Curzen N. Non-invasive Fractional Flow Reserve using Computed Tomographic angiography: where are we now and where are we going? Heart. 30 May 2017. doi: 10.1136/heartjnl-2016-311029

2. Modi BN, Van de Hoef TP, Piek JJ, Perera D. Physiological Assessment of Left Main Coronary Artery Disease. EuroIntervention. 2017 Jun 13. pii: EIJ-D-17-00135. doi: 10.4244/EIJ-D-17-00135.

3. Rahman H, Modi B, Ellis H, Arri S, Perera D. Lead to the Culprit. Circulation August 2017. Circulation. 2017;136(9):877-879. doi:10.1161/circulationaha.117.029802.

4. Modi BN, Rahman H, Sherif SA, Ellis H, Eruslanova K, Chiribiri A, Perera D. Is heart rate response a reliable marker of adenosine-induced coronary hyperaemia? Int J Cardiovasc Imaging 2018. doi:10.1007/s10554-018-1309

5. Modi BN, De Silva K, Rajani R, Curzen N, Perera D. Physiology-Guided Management of Serial Coronary Artery Disease. JAMA Cardiology. 2018;:1-3

6. Claridge S, Briceno N, Chen Z, De Silva K, Modi B, Jackson T, Behar J, Niederer S, Rinaldi CA, Perera D. Changes in contractility determine coronary haemodynamics in dyssynchronous left ventricular heart failure, not vice versa. IJC Heart \& Vasculature 2018.19IS-:8-13.

7. Modi BN, Chattersingh A, Eruslanova K, Ryan M, Ellis H, Gaddum N, Chowienczyk P, Perera D. Optimal Application of Fractional Flow Reserve to Assess Serial Coronary Artery Disease: 3-D Printed Experimental Study with Clinical Validation. Accepted for publication in the Journal of the American Heart Association (JAHA) 2018.

DOI:10.1161/JAHA.118.010279 
8. Modi BN, Rahman H, Arri S, Ellis H, Mills MT, Willias R, Asrress K, Clapp B, Redwood S, Perera D. Resting Coronary Flow Varies With Normal Cardiac Catheter Laboratory Stimuli. Accepted for publication in Cardiovascular Revascularisation Medicine. 2018.

9. Modi BN, Rahman H, Kaier TE, Ryan M, Williams R, Briceno N, Ellis H, Pavlidis A, Redwood S, Clapp B, Perera D. Revisiting the Optimal FFR and iFR Thresholds for Detecting Significant Coronary Artery Disease. Circulation: Cardiovascular Interventions 2018;11:e07041. DOI: 10.1161/CIRCINTERVENTIONS.118.007041

10. Modi BN, Rahman H, Ryan M, Ellis H, Pavlidis A, Redwood S, Clapp B, Chowienczyk P, Perera D. Physiological Characterization of Serial Coronary Artery Disease: A Comparison of Currently Available Pressure and Resistance-based Indices and a Novel Mathematical Solution. Submitted to the European Heart Journal.

11. Modi BN, Sankaran S, Kim HJ, Ellis H, Rogers C, Taylor CA, Rajani R, Perera D. Predicting the Physiological Effect of Revascularization in Serially Diseased Coronary Arteries: Clinical Validation of a Novel CT Coronary Angiography-Based Technique.

Circulation: Cardiovascular Interventions. 2019;12:e007577.

DOI:10.1161/CIRCINTERVENTIONS.118.007577

\subsection{ABSTRACTS}

1. How Physiological is Adenosine-Induced Hyperaemia? Insights From Exercise in the Catheterisation Laboratory. Matthew Ryan, Bhavik Modi, Satpal Arri, Kaleab Asrress, Matthew Lumley, Rupert Williams, Howard Ellis, Brian Clapp, Michael Marber, Simon Redwood, Divaka Perera. Presented at American Heart Association Congress, New Orleans 2016. Circulation. November 2016;134:A19777

2. Optimising physiological assessment of serial coronary artery lesions using an in vitro model of tandem stenoses, B. Modi, A. Chattersingh, M. Ryan, H. Ellis, J. Lee, N. Gaddum, P. Chowienczyk, D. Perera. EHJ, Vol 38, suppl_1, Aug 2017 
3. Optimising Physiological Assessment of Serial Coronary Artery Lesions Using an in vitro 3-D Printed Model of Tandem Stenoses. Bhavik N Modi, Anjalee Chattersingh, Kseniia Eruslanova, Matthew Ryan, Howard Ellis, Jack Lee, Phil Chowiencyzk, Divaka Perera. Circulation. 2017;136:A17271

4. Optimising physiology guided management of serial coronary artery disease. Bhavik Modi British Cardiovascular Intervention Society, Young Investigator Award Shortlisted Presentations, Royal College of Physicians of London, November 30 2017Heart (British Cardiac Society) 104(Suppl 1):A2-A3. Jan 2018. DOI 10.1136/heartjnl-2018-BCIS.3

5. Instantaneous Wave-Free Ratio/Fractional Flow Reserve Discordance: Is The 'WaveFree Period' Truly 'Wave-Free'? TCT-151. 2018;72(13):B64-B65.

doi:10.1016/j.jacc.2018.08.1260. Presented at TCT, September 2018. San Diego, USA

6. Clinical Utility of a Novel Mathematical Solution for Assessment of Individual Lesion Contribution in Serial Coronary Artery Disease. TCT-466. 2018;72(13):B187. doi:10.1016/j.jacc.2018.08.1637. Presented at TCT, September 2018. San Diego, USA

\subsection{AWARDS}

2015-2018: Clinical Research Training Fellowship (British Heart Foundation FS/15/78/31678). Awarded $£ 211,153$ for 'Fractional flow reserve in serial and diffuse coronary artery disease'

2016: Finalist, Young Investigator of the year, EuroPCR congress, Paris.

2017: Winner, Annual Research Day Competition, British Cardiovascular Intervention Society, Royal College of Physicians, London

2018: Finalist, Young Investigator of the year, British Cardiovascular Intervention Society, ACI Congress, London 


\section{BACKGROUND}

Published in JAMA Cardiology, Heart and Eurointervention

\section{Acknowledgements}

I would like to pay particular gratitude to the following people for their help with this section:

Dr Matthew Lumley (Previous BHF Clinical Research Fellow)

Dr Kalpa De Silva (Previous BHF Clinical Research Fellow)

Prof Phil Chowienczyk (Secondary Supervisor)

Prof Divaka Perera (Primary Supervisor) 
There is growing evidence that the greatest benefit of revascularisation for coronary artery disease $(\mathrm{CAD})$ is derived from targeting myocardial ischaemia. Traditionally, functional assessment to identify patients with CAD was based on non-invasive tests prior to angiography ${ }^{1}$. This has subsequently evolved to identifying vessels with functionally significant disease at the time of angiography, such as with Fractional Flow Reserve (FFR) and Instantaneous Wave-Free Ratio (iFR) $)^{2-4}$.

$\mathrm{CAD}$ is the result of atherosclerosis, which is systemic in nature, and therefore an element of serial/diffuse disease is common, with estimates suggesting a prevalence of $25-40 \%$ in all angiograms ${ }^{5,6}$. There is often a need to identify the physiological significance of individual lesions when deciding on a revascularisation strategy, but this is potentially challenging because of physiological interplay between serial stenoses that alters the apparent significance of each lesion.

The background to this thesis describes the basis of ischaemia-guided revascularisation and the tools with which we assess the physiological significance of stenoses. I will describe the physiology of serial stenoses and the problems posed when judging the physiological significance of individual stenoses. I will go on to discuss current and emerging techniques that may be used to assess the functional significance of individual lesions when planning revascularisation strategies. 


\subsection{ASSESSING STENOSIS SEVERITY DURING ANGIOGRAPHY}

Traditionally, the extent of coronary artery disease (CAD) has been assessed using the invasive coronary angiogram. However, there is growing evidence that the benefit of revascularisation, above and beyond optimal medical therapy, is derived from targeting myocardial ischaemia ${ }^{7}$ Traditionally, functional assessment to identify patients with CAD was based on non-invasive tests prior to angiography ${ }^{1}$. This subsequently evolved to identifying vessels with functionally significant disease at the time of diagnostic angiography using invasive flow-based indices such as Coronary Flow Reserve (CFR) and Invasive Pressure-Flow based resistance indices, such as Hyperaemic Stenosis Resistance (hSR) and Basal Stenosis Resistance (BSR). The most recent iterations of ischaemia testing on a per-vessel basis have come in the form of pressure-derived indices of ischaemia and coronary flow-impairment, with tools such as Fractional Flow Reserve (FFR) and Instantaneous Wave-Free Ratio (iFR) $)^{2-4}$ (See figure 5.1).

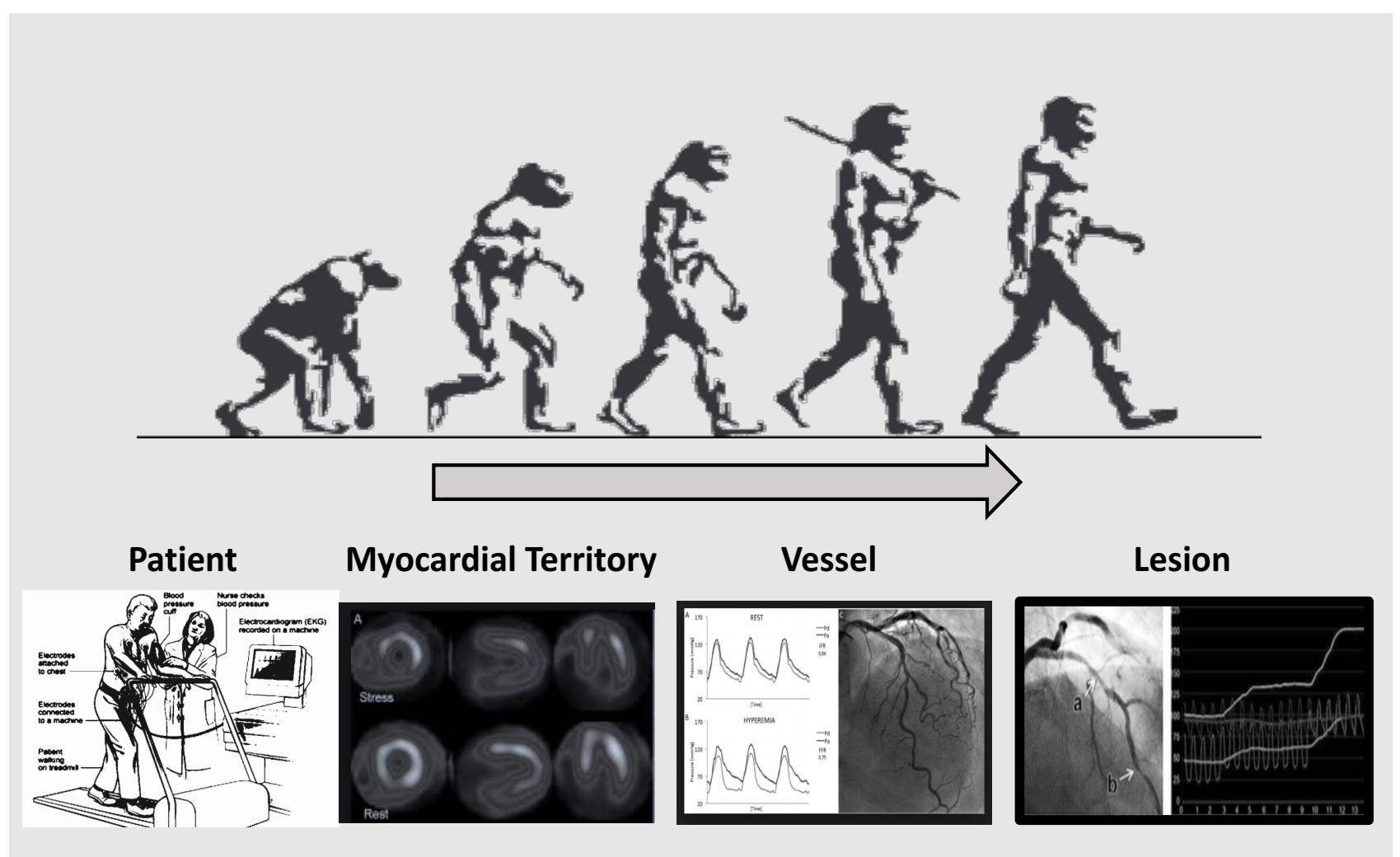

Figure 5.1: An illustration of the evolution of ischaemia testing with a question remaining about its evolution to lesion-specific assessment, particularly in the presence of serial disease 


\subsubsection{Fractional Flow Reserve, FFR}

The pressure drop in a vessel (ratio of distal coronary to aortic pressure, $\mathrm{Pd} / \mathrm{Pa}$ ) during maximal adenosine-induced hyperaemia, when the reduction in flow in the distal vessel is assumed proportional to the change in pressure across it, is referred to as Fractional Flow Reserve (FFR) and is the most widely used pressure index of vessel ischaemia. FFR was initially validated over two decades ago against non-invasive surrogate markers of ischaemia in the setting of stable angina ${ }^{8}$. Since then, there has been increasing evidence demonstrating that visual assessment of angiographic stenosis severity is prone to significant error and does not reliably correlate to underlying functional significance, leading to a misclassification of stenoses in around a third of lesions $^{1,9}$. As a consequence, Ischaemia-guided revascularisation with FFR appears to confer significant clinical and prognostic benefit over management based on angiography alone, particularly for stable angin ${ }^{2,4,10-12}$, but also the non-culprit NSTEMI settings ${ }^{13}$.

\subsubsection{Resting Indices of Stenosis Severity: Resting PdPa \& iFR}

\section{Resting Pd/Pa Ratio}

The ratio of whole cycle distal coronary pressure to aortic pressure at rest $(\mathrm{Pd} / \mathrm{Pa})$ was originally used by Gruentzig to rationalise balloon angioplasty decisions ${ }^{14}$. Although clinical outcome data is absent, one study found accuracy of $>80 \%$ when compared against $\mathrm{FFR}^{15}$ and recent subgroup analyses of the VERIFY and CONTRAST studies suggested resting Pd/Pa and iFR correlate almost perfectly ${ }^{16,17}$. With more and more data emerging to show near perfect agreement of iFR and $\mathrm{PdPa}^{17}$, there is drive to suggest using it in a similar fashion to iFR with a threshold of $\mathrm{PdPa} \leq 0.91$ suggested to correlate excellently with $\mathrm{IFR} \leq 0.89^{17}$.

$i F R$

iFR is defined as average $\mathrm{P}_{d} / \mathrm{P}_{\mathrm{a}}$ during the latter $75 \%$ of diastole (minus the last $5 \mathrm{~ms}$ ), when resistance is purported to be constant and pressure can be assumed proportional to flow, without the need to modulate resistance with adenosine-induced hyperaemia ${ }^{7}$. iFR-guided management 
has recently been shown to have comparable investigator determined revascularisation rates to FFR, in a relatively low-risk group of patients, with data awaited on more complex subsets ${ }^{11,12}$.

\section{New Resting Indices of Stenosis Severity}

Recently, resting pressure-derived indexes such as resting full-cycle ratio (RFR) and diastolic pressure ratio $(\mathrm{dPR})$ have been introduced to assess the functional significance of epicardial coronary stenosis. Evidence is accumulating to suggest all resting indices are equivalent (iFR, $\mathrm{RFR}, \mathrm{dPR}$ and $\mathrm{Pd} / \mathrm{Pa}$ ) with a similar diagnostic accurancy ${ }^{18}{ }^{19}$. Infact it has been suggested that all these indices could well be grouped under the title: 'Non-Hyperaemic Pressure Ratios (NHPR)'.

\subsubsection{Pressure-Flow indices of Stenosis Severity: CFR \& HSR}

\section{CFR}

Coronary Flow Reserve (CFR) was one of the first measures of stenosis severity. CFR is defined as the ratio of baseline to hyperaemic flow in a coronary artery. CFR takes into account both the epicardial and microvascular resistance. An impaired CFR has been shown to be correlated with worse prognosis and a state of 'low flow ischaemia'20.

Measuring CFR requires us to measure intracoronary flow either by Doppler-tipped guidewires or by thermodilution techniques. Given CFR cannot differentiate between epicardial and microvascular coronary resistance, it is not a stenosis specific marker and cannot therefore be used to identify the functional significance of individual stenoses in serial disease. Dopplerbased measurements of flow, originally utilized to measure CFR, can however be used to measure resistance.

\section{Resistance Indices}

Once flow is measured reliably, establishing the resistance of an individual stenosis becomes possible. The 'resistance' of an individual lesion should theoretically be an individual property 
of a stenosis and independent of pressure and flow conditions within a simple vessel. The resistance of an individual stenosis, by analogy with Ohm's Law is given by the change in Pressure across it $(\Delta \mathrm{P})$ divided by the Flow Velocity through the vessel $(\mathrm{Q})$. This ratio in hyperaemic conditions is termed the 'Hyperaemic Stenosis Resistance' (hSR). The hSR of a stenosis has been shown to be more accurate than FFR and CFR at detecting reversible perfusion defects seen using non-invasive modalities ${ }^{21}$, has been shown to be better than its equivalent measured at rest ('Basal Stenosis Resistance', BSR) 22 and is often considered by some to represent the 'gold standard' for ischaemia resulting from an individual stenosis.

The resistance (hSR) of a lesion should theoretically be an independent property of a stenosis, independent of flow conditions and disease elsewhere within a vessel: much like a resistor within an electrical circuit. Whether the hSR of a stenosis does indeed behave in this fashion is yet to be established in the form of data. If it does, it may be an ideal tool with which to assess the true significance of individual lesions in the presence of serial coronary artery disease.

\subsection{THE PROBLEM OF SERIAL CORONARY ARTERY DISEASE}

As described in section 5.1, serial/diffuse disease is common, with estimates suggesting a prevalence of $25-40 \%$ in all patients ${ }^{5,6}$. In the presence of serial disease, that an interventional cardiologist would consider treating separately, there is the need to identify the physiological significance of individual lesions. This is a challenge and an unmet clinical need is because of physiological interplay that alters the apparent functional severity of each lesion leading to potential error.

\subsubsection{Factors Influencing the Pressure Drop Across A Stenosis}

To understand serial stenosis interplay, we must first understand the factors that lead to pressure changes across a lesion $(\Delta \mathrm{P}) . \Delta \mathrm{P}$ is determined by several factors that are governed by principles 
enshrined in the Bernoulli equation and Poiseuille's Law. As summarised in figure 5.2, $\Delta \mathrm{P}$ across a stenosis, as demonstrated in seminal canine experiments ${ }^{23}$, can be summarised as $\Delta \mathrm{P}=$ $\mathrm{fQ}+\mathrm{sQ} \mathrm{Q}^{2}$, where $\Delta \mathrm{P}=$ change in pressure, $\mathrm{f}=$ the frictional coefficient of a lesion, $\mathrm{s}=$ the separation coefficient of a lesion and $\mathrm{Q}=$ Blood Flow through the stenosis. This relationship, including both the frictional and separation coefficients, embodies the Bernoulli principle and Poiseuille's Law and is determined largely by specific lesion geometry. Aside from how the specific shape of a stenosis affects these coefficients, it is worth considering the other important factors:

- $\quad$ Luminal Narrowing: This contributes to both frictional (f) and separation coefficient (s) of a lesion. According to Poiseuille's law, $\Delta \mathrm{P}$ across a stenosis is the product of volumetric flow rate and viscous resistance $(R)$, with the latter defined as $R=8 \eta L / \pi r^{4}$, where $\eta$ is the viscosity of the fluid, $L$ is the length of the vessel, and $r$ is the radius of the vessel ${ }^{24}$. Although blood flow in smaller vessels may depart from this relationship, for most epicardial vessels under a physiological range of pressures, resistance and hence $\Delta \mathrm{P}$, varies inversely with the fourth power of vessel radius.

- $\quad$ Lesion Length: This contributes linearly to viscous resistance (by Poiseulle's Law) and to the viscous coefficient of the equation in figure 5.2. Although the impact on $\Delta \mathrm{P}$ may not be as profound as the degree of stenosis, lesion length is important, especially in long diffusely diseased segments.

- $\quad$ Flow Conditions: In fluid dynamics, laminar flow is "orderly" and turbulent flow is "random". Within a pipe, flow is laminar, however in the presence of a stenosis, flow separation increases as eddy currents begin to form and flow becomes non-laminar beyond a certain threshold (the 'Reynolds Number') with subsequently increased flow separation ${ }^{25}$. Such changes in flow conditions form the basis of the flow separation coefficient in figure 5.2.

- $\quad$ Flow Velocity: This factor is influenced by changes in downstream resistance. We know from work by Uren et al that as a stenosis is removed, resistance falls and hyperaemic flow increases $^{26}$ and as inlet flow velocity changes for any remaining stenosis, so will $\Delta \mathrm{P}$ across it. 


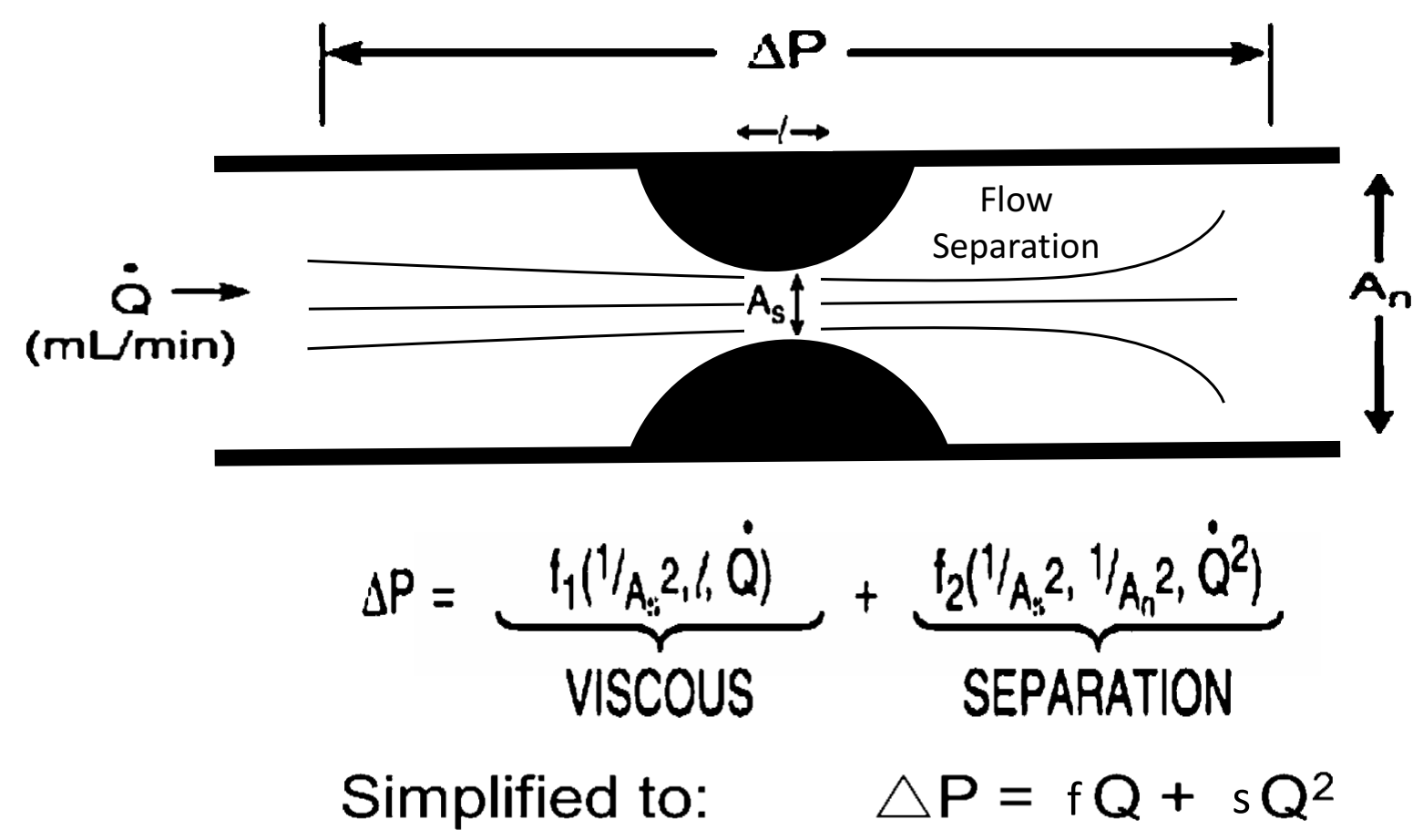

Figure 5.2: Factors Influencing the Pressure Gradient Across a Stenosis. Pressure gradients across a stenosis can be described by a relationship from the energy losses by viscous friction and flow separation ${ }^{23}$, which takes the form $\Delta \mathrm{P}=\mathrm{fQ}+\mathrm{sQ}^{2}$, where $\Delta \mathrm{P}=$ change in pressure, $\mathrm{f}=$ frictional coefficient of a lesion (influenced by lesion length and diameter stenosis), $\mathrm{s}=$ separation coefficient of a lesion (influenced by diameter stenosis and the laminar flow separation) and $\mathrm{Q}=$ flow

\subsubsection{The unique physiology of serial stenoses}

Based on these principles described in the previous section, any significant accompanying disease will, in turn, increase total resistance, regardless of whether that disease is upstream or downstream of the stenosis in question. Therefore, it follows that the significance of stenosis may be artificially underestimated by pressure-based indices (Figure 5.3). 

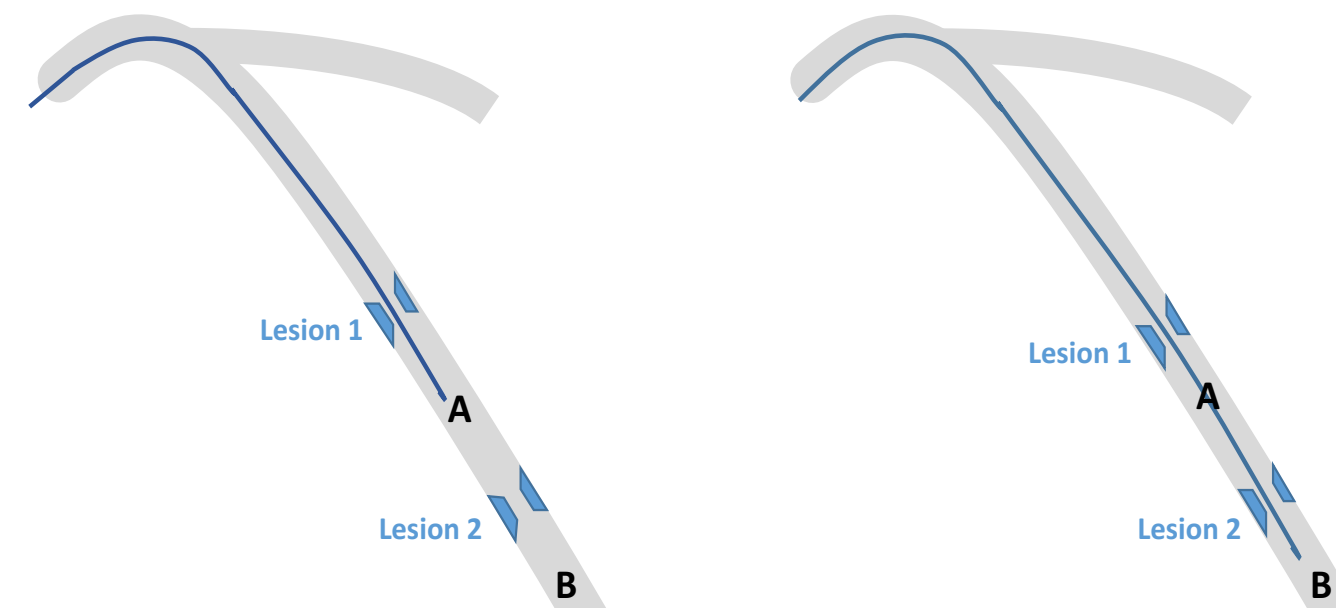

Pressure Wire Placed Between Lesions

$\triangle \mathrm{P}$ of lesion 1 is likely to be underestimated

Pressure Wire Placed Between and then Distal to Both Lesions

$\triangle P$ of lesion 2 likely to be underestimated

The smaller the distance between lesions, the greater the potential for flow turbulence and further error

Figure 5.3: Serial Stenosis Interplay. Diagrammatic representation of how serial stenosis interplay can result in erroneous results from a pressure wire. Both lesions contribute to total vessel resistance. In absence of either lesion, flow is greater and therefore $\Delta \mathrm{P}$ across the remaining lesion is likely to be underestimated.

The presence of stenoses elsewhere, particularly if closer together, can also alter flow conditions significantly such that laminar flow becomes more turbulent with subsequent energy loss (and therefore pressure). The degree to which altered flow conditions influence $\Delta \mathrm{P}$ depends on the individual frictional (f) and separation (s) coefficients of the stenoses within the serially diseased vessel (Figure 5.2). In a serially diseased vessel, $\Delta \mathrm{P}$ and thus the individual contribution of an individual stenosis to overall FFR, are therefore likely to be determined by not just $\Delta \mathrm{P}=\mathrm{fQ}+\mathrm{sQ}^{2}$ but also total vessel resistance and the alteration of these laminar fluid dynamics.

\subsubsection{Theoretical solutions to overcome the FFR interplay of serial stenoses}

Various theoretical solutions have been examined to define an algorithm for determining the individual contribution of each stenosis to total vessel FFR ('FFR ${ }_{\text {true }}$, were each stenosis present in isolation). Such a solution was determined in vitro and subsequently tested in canines by De 
Bruyne et al to show that $\mathrm{FFR}_{\text {predicted }}$ from the theoretical equation made some improvements in identifying FFR $_{\text {true }}{ }^{27}$. This method required knowledge of coronary occlusive wedge pressure between stenoses, whereby:

$$
\text { predicted FFR }=(\mathbf{P d}-[(\mathbf{P m} / \mathbf{P a}) \times \mathbf{P w}]) /([\mathbf{P a}-\mathbf{P m}]+[\mathbf{P d}-\mathbf{P w}])
$$

Where: $\mathrm{Pm}$ is the pressure between the 2 stenoses, $\mathrm{Pd}$ is the most distal pressure, $\mathrm{Pw}$ is the wedge pressure (all measured during maxi- mum hyperaemia)

This solution was subsequently validated in a small clinical study by Pijls et al, where a pressure wire was used as a standard angioplasty wire in the assessment of 32 tandem lesions, enabling a wedge pressure measurement during ballooning of each lesion. They found improved accuracy in identifying the attributable FFR true of individual lesions ${ }^{28}$.

This technique, whilst well validated, is difficult to use in clinical practice and considered impractical and cumbersome ${ }^{29}$ because it involves ballooning arterial segments to measure a wedge pressure ${ }^{30}$, without having first determined an appropriate revascularisation strategy.

\subsection{CURRENT METHODS IN PHYSIOLOGICAL MANAGEMENT OF SERIAL AND DIFFUSE CORONARY ARTERY DISEASE}

\subsubsection{Ignoring the Problem}

The presence of serial stenosis interplay is often ignored, with measurement of intracoronary pressure between, and distal to, sequential lesions being used to erroneously estimate what each lesion contributes to total FFR, with significant theoretical limitations (Figure 5.3). Measuring intracoronary pressure between lesions (without ballooning to measure wedge pressure) disregards the fact that total vessel resistance is greater in the presence of an accompanying stenosis (with subsequent underestimation of pressure gradients). Furthermore, the presence of 
an accompanying lesion, particularly up to a certain distance apart, also impacts flow conditions and turbulence within the vessel (contributing to coefficient 's' in Figure 5.2).

\subsubsection{The 'Educated Guess'}

Another commonly adopted, but imperfect solution, is to make a visual 'educated guess' regarding which segment is most significant, in the presence of a vessel with FFR $\leq 0.80$, and treating this first. Following initial PCI, the FFR is repeated, with further PCI performed if FFR $\leq 0.8$. This is based on an interpretation of data by Pijls et al, who, in a 750-patient registry, showed that FFR after stenting was a significant predictor of events at 6 months. The study showed that patients with post-PCI FFR $>0.95$ had an event rate of $4.9 \%$; whereas in the group with post-PCI FFR of $0.80-0.90$, event rate was $29.5 \%(p=0.001)^{31}$. Whilst these data support ensuring the best possible physiological result, they do not tell us how to achieve this with serial disease. By attempting to identify the lesion that has the "most functional significance", there is a possibility that an accompanying lesion is under-estimated and therefore the wrong revascularisation strategy is chosen, for example if a Left Main Coronary Artery (LMCA) stenosis is mistakenly underestimated. A 'lesion-specific' physiological strategy, that counteracts serial stenosis interplay, would therefore be theoretically superior to a strategy that combines whole vessel FFR with an angiographic visual 'guess' of the contribution of individual segments.

\subsubsection{FFR Pullback Manouvre}

Pulling back a pressure wire under fluoroscopy is increasingly performed to assess for increments in coronary pressure $(\Delta \mathrm{P})$, with the assumption that $\Delta \mathrm{P}$ is indicative of lesion significance. This method, illustrated in figure 5.4, was examined by two observational studies $^{32,33}$, in which PCI strategy was guided by stenting the stenosis with the greatest $\Delta \mathrm{P} . \mathrm{Kim}$ et al found that in 131 stable patients with total vessel $\mathrm{FFR} \leq 0.80$, PCI was safely deferred in 
$61.1 \%$ of lesions based on FFR after initial targeted PCI with no clinical events related to deferral. Park et al studied 52 patients (104 lesions) and found the practice of treating the lesion with greatest ' $\Delta \mathrm{P}$ ', re-measuring FFR and carrying out further PCI until FFR $>0.8$ is also associated with good clinical outcomes ${ }^{32}$.
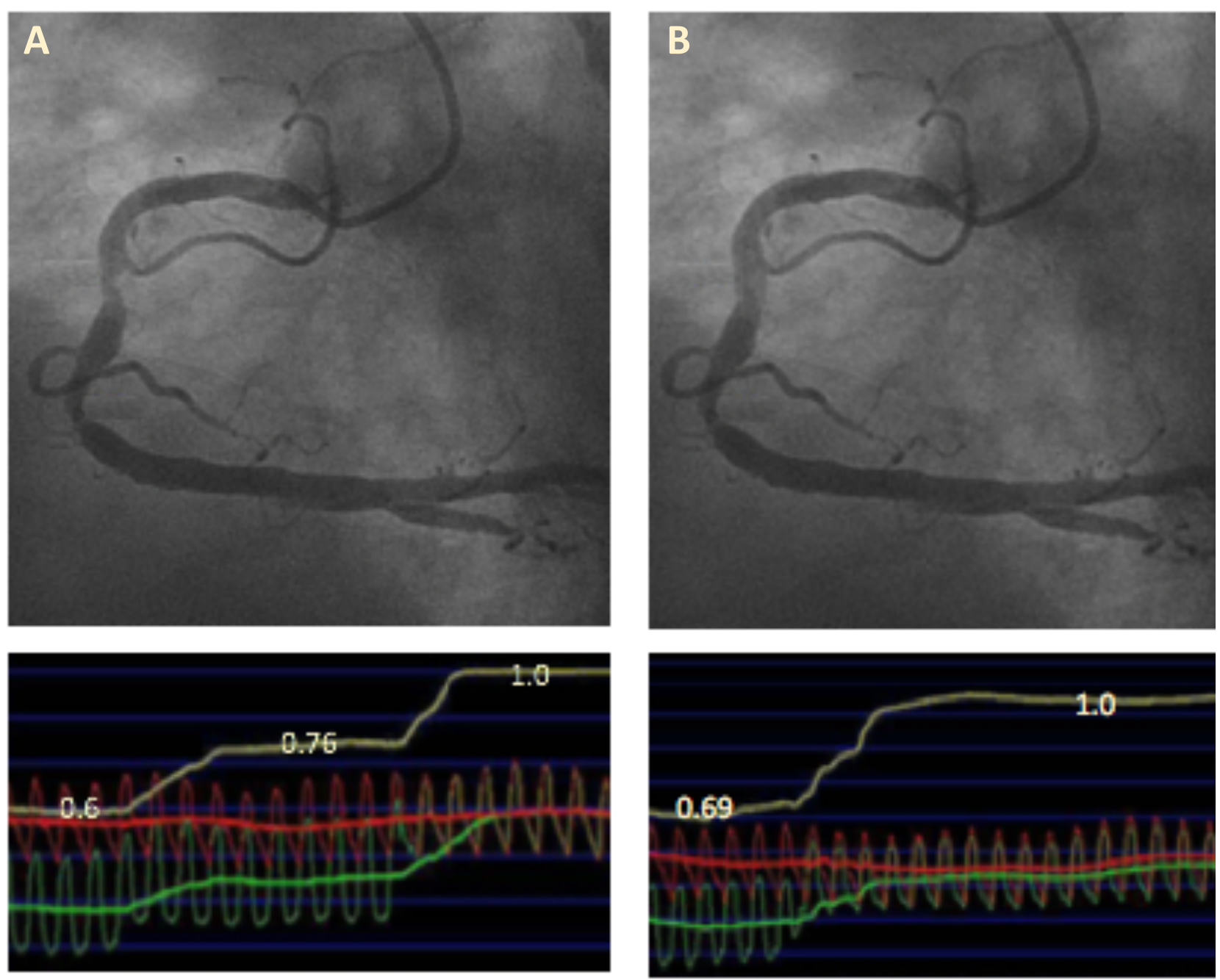

Figure 5.4: The Pressure-Wire Pullback Manoeuvre. Representation of manual pullback method in a Right Coronary Artery with the FFR change seen on the console at each point. The largest pressure gradient $(\Delta \mathrm{P})$ corresponds to proximal lesion on image A with an 'apparent' $\Delta \mathrm{FFR}$ attributable to the distal lesion of 0.16 . Upon treating the proximal lesion (image B), a repeat pullback reveals the $\Delta \mathrm{P}$ of the distal lesion was originally underestimated (the 'true' $\Delta \mathrm{FFR}$ attributable to the lesion being 0.31).

The method of treating the greatest $\Delta \mathrm{P}$ following manual pullback does however have potential pitfalls:

- $\quad$ Although underestimation is more common, rarely overestimation is possible: this has been observed in several studies showing ' $\triangle F F R_{\text {apparent }}$ ' of lesions is on rare occasions greater 
than the ' $\Delta F F R_{\text {true }}{ }^{27,30,32,33}$. A potential reason for this is that increased flow turbulence in serial disease may be more influential than the increased vessel resistance that usually causes underestimation. Overestimation means stenting the largest ' $\Delta \mathrm{P}$ ' could result in stenting functionally non-significant stenoses.

- $\quad$ Treating the greatest ' $\Delta \mathrm{P}$ ' following manual pullback has potential for operator error. For example, there is natural tendency to pause pullback for fluoroscopic guidance, half-way through identifying a gradient. This can result in artefactual plateaus with misinterpretation of one pressure gradient as two. Additionally, operators may only pull back through segments they believe visually significant ${ }^{1,9}$.

Without the ability to accurately assess the true physiological contribition of each lesion at the outset, it may be that after treating the lesion contributing the greatest ' $\Delta \mathrm{P}$ ', a physiologically significant lesion will still remain.

\subsection{4 iFR Pullback}

As described earlier, iFR is a resting pressure-wire based index of coronary blood flow impairment that doesn't require the need to modulate resistance with adenosine-induced hyperaemia ${ }^{7}$ with iFR-guided revascularisation recently shown to have comparable outcomes to FFR, in a relatively low-risk group of patients ${ }^{11,12}$.

It has been suggested that serial stenosis interplay and alteration in flow conditions may be amplified in hyperaemic conditions. This is based on an interpretation of canine experiments by Gould et al that demonstrated the $\Delta \mathrm{P}=\mathrm{fQ}+\mathrm{sQ}^{2}$ relationship. These experiments showed that for a fixed lesion, when flow transitioned from rest to hyperaemic flow, the pressure-velocity gradient increased ${ }^{23,34}$. Proponents of iFR therefore argue that serial stenosis interplay is potentially less at rest, with iFR in particular having added spatial resolution advantages over $\mathrm{Pd} / \mathrm{Pa}$, since it isn't calculated from pressures averaged over several cardiac cycles and diastolic 
resistance may be lower than whole cycle resistance. On this basis, iFR has been used to study serial disease in a small cohort of 32 coronary arteries ${ }^{35}$. In this study, pressure wire pullback at rest was used to demonstrate how iFR changed along each artery; this was then used to virtually remove the accompanying lesion, with the residual 'expected iFR' correlating well with 'observed iFR' after PCI (incidental resting $\mathrm{Pd} / \mathrm{Pa}$ was not assessed in this study). This led to the development of the 'iFR Scout' software (Volcano, Phillips Inc), based on the manual pullback maneuver performed for FFR (Figure 5.4), and aims to predict the residual iFR were one lesion

removed and presumes that the change in iFR across a stenosis remains unchanged regardless of co-existent disease. A subsequent multi-centre utility study has shown the clinical utility of this software ${ }^{36}$ but the comparative value of iFR both on clinical outcomes and versus other physiological indices in serial disease is yet unknown. The value of iFR pullback has also not yet been validated in large-scale studies of a more severe spectrum of serial disease when stenosis interplay is likely to be more significant.

This thesis includes a study that systematically tests the hypothesis that serial stenosis interplay is lessened at rest. As such evidence supporting or refuting resting indices grows, there will be an opportunity to establish for certain whether resting indices are more accurate in serial/diffuse disease. In the meantime, $\mathrm{iFR}$ in serial disease has the potential to provide a solution to overcome some of the difficulties in assessing the relative lesion significance in serially diseased vessels; we eagerly await further data, including our own, to support this notion.

\subsection{PHYSIOLOGY GUIDED MANAGEMENT OF LEFT MAIN CORONARY ARTERY DISEASE: AN IMPORTANT EXAMPLE OF SERIAL STENOSIS INTERPLAY}

\subsubsection{Evidence for FFR Assessment of the LMCA}

The seminal validation study of FFR for the identification of myocardial ischaemia was performed in stable patients against a combination of non-invasive ischaemia tests, but only enrolled two patients with LMCA disease ${ }^{8}$. Subsequently, evidence has grown from trials 
demonstrating improved outcomes by FFR-guided-PCI versus angiography alone: again these have all excluded LMCA disease ${ }^{2,10,4}$. The reasons excluding LMCA stenoses is unclear but is almost certainly contributed to by the complexities of physiologically assessing LMCA stenoses when present alongside serial stenoses in downstream daughter vessels.

Despite this evidence continues to mount to suggest that physicians are poor at identifying physiologically significant lesions by angiography alone ${ }^{1,37}$. A recent study of over 4000 coronary lesions with both angiography and FFR, found that coronary angiography alone misestimates physiological severity when using a FFR threshold of 0.8 in over a third of angiographically intermediate stenoses ${ }^{1}$. This observational study included 152 patients with LMCA and found visual-functional mismatch to be just as common, with a particularly high lesion underestimation. Hamilos et al reported the largest observational study on the utility of FFR in LMCA lesion assessment ${ }^{38}$ : patients with angiographically equivocal LMCA disease who underwent adjunctive pressure wire assessment were enrolled. Patients with FFR $>0.80$ were managed with medical therapy and those with a FFR $\leq 0.80$ underwent CABG. They found equivalent clinical outcomes in both groups, suggesting an LMCA FFR threshold of 0.80 may be used to defer revascularisation. A recent meta-analysis of observational data (525 patients from 6 studies) drew similar conclusions ${ }^{39}$.

Whilst significant observational data suggests FFR-guided revascularisation should be performed for the LMCA, several criticisms can be made of current observational data. The studies didn't use a uniform FFR threshold, hampering extrapolation to contemporary practice. In addition, observational data is prone to the pitfalls of non-randomized data, including selection bias, which cannot be overcome by meta-analyses. One of the arguments against the use of FFR to guide LMCA revascularisation is that the risk of plaque rupture events may not be related to the haemodynamic significance of a stenosis but is instead determined by underlying plaque composition. In other words, deferring revascularisation of the LMCA on the basis of a FFR $>0.80$ may leave a group of patients at risk of myocardial infarction. Although there has 
been no signal of excess MI/death in the vast majority of FFR trials, the recently presented FUTURE trial (NCT01881555) did include LMCA disease in 11\% of patients, with a signal for excess mortality in the FFR-guided arm (although it is only speculation to suggest the events came from deferred LMCA stenoses). Whilst we await robust randomized data on FFR in LMCA disease, the FUTURE trial data should make us wary of the potential consequences of LMCA plaque rupture compared to a more distal plaque rupture event, and the observational FFR data of LMCA revascularisation to date are likely underpowered to detect this.

\subsubsection{Theoretical Challenges to Physiological Assessment of the LMCA}

Coronary blood flow is related to the size of the viable myocardial bed subtended ${ }^{40}$. FFR takes account of the myocardial mass perfused as it is the ratio of blood flow to a given mass of tissue in the presence of a stenosis $\left(\mathrm{Q}_{\mathrm{s}}\right)$ to blood flow to the same mass of tissue if the vessel were unobstructed $\left(\mathrm{Q}_{\mathrm{N}}\right)$ : whereby $\mathrm{FFR}_{\mathrm{myo}}=\mathrm{Qs}_{\mathrm{s}} \mathrm{Q}_{\mathrm{N}}$. The LMCA territory constitutes the vascular beds of the left anterior descending (LAD) and left circumflex (LCx) arteries ${ }^{41}$ and, in the absence of obstructive disease in each branch, the ratio of flow in each is determined by the resistances of their respective vascular beds. As the minimal resistance in a vascular bed can be assumed to be independent of any upstream stenosis ${ }^{42}$, the FFR of a LMCA stenosis should be identical, whether measured in the LCx or LAD. However, in the presence of any downstream disease, FFR will be determined by the LMCA stenosis as well as disease in each limb. Given that atherosclerosis tends to affect the whole coronary tree, LMCA disease is usually accompanied by additional downstream disease in the LAD and/or $\mathrm{LCx}^{43}$. In such cases, the LMCA and distal stenosis should be considered to act as serial stenoses ${ }^{30,44}$.

As discussed, ischaemia assessment of individual lesions in the presence of serial stenoses is problematic because of the complex inter-dependence of each lesion that affects their relative severity, even when there is a disease-free sidebranch (e.g. LMCA stenosis co-existing with a stenosis in the mid-LAD but with a disease-free $\mathrm{LCx}$ ). The presence of disease distal from the 
LMCA is generally associated with underestimation of stenosis severity, and may lead to inadvertent deferral of LMCA revascularisation ${ }^{33}$. The potential influence of distal disease is frequently ignored when drawing conclusions on the functional significance of LMCA stenoses with significant consequences in this critical setting.

Whilst potential interaction is recognized, the practice of measuring LMCA FFR by placing the wire in the disease-free daughter vessel has been shown to be a reliable method in in vitro studies with only minimal interference from downstream disease in the diseased-branch ${ }^{44-46}$. Fearon et al went on to demonstrate this in their in vivo study by interrogating LMCA stenoses in 25 patients following PCI of the LAD+/-LCx. FFR was measured in the LAD and LCx before and after creation of downstream lesions by inflating balloons within newly-placed stents. They then compared 'true' FFR measured in the disease-free vessel and compared this with the 'apparent' FFR measured in the diseased-vessel. They found the true LMCA FFR was significantly lower than apparent FFR (0.81vs.0.83, $\mathrm{p}<0.001)$, although the authors interpreted this as clinically insignificant and at the upper-end of the natural test-retest variability of FFR. From this relatively small study, the authors concluded that in most cases when there is a disease-free branch of the LMCA, downstream disease within the other branch doesn't have a clinically significant impact on LMCA FFR with the pressure wire positioned in the disease-free vessel, unless disease within this branch is very severe (FFR $<0.45$ ), and that an FFR value of $>0.85$ in the disease-free sidebranch would mean the LMCA lesion can be safely assumed functionally non-significant ${ }^{44}$. Whilst this seems reassuring, there remains concern that any significant difference in the continuous variable of FFR may be associated with important outcome differences. Whilst these studies are small and prone to theoretical flaws, for the purpose of LMCA disease with serial disease in only 1 daughter vessel, this method is, for now, an acceptable solution for this unique case of serial stenosis interplay (illustrated in figure 5.5) 


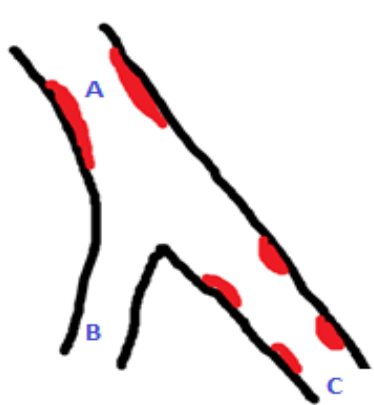

Left Main Stenosis (A) with Further Serial

Stenoses in LAD (C)

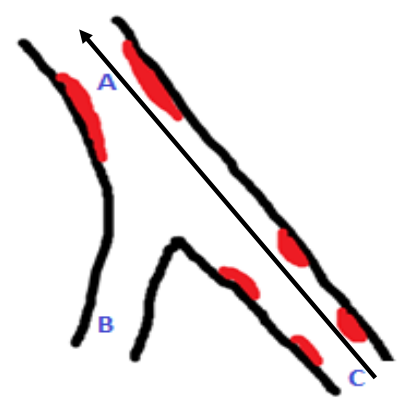

Pressure-Wire Pullback from Diseased LAD (C)

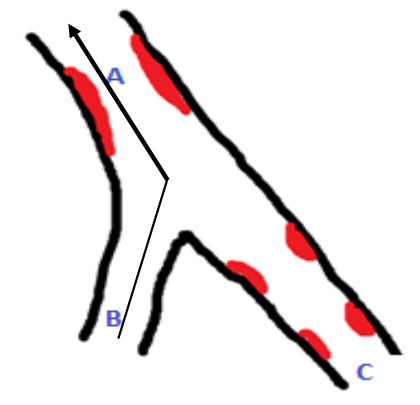

Pressure-Wire Pullback from Disease-Free

Circumflex (B)

Figure 5.5: Illustration of the disease-free sidebranch method to isolate the true significance of the Left Main stenosis when accompanied by serial disease in one downstream vessel (in this case the Left Anterior Descending, LAD)

\subsubsection{Best Practice in Left Main Physiological Recordings}

\section{General Technical Considerations}

Physiological assessment in general, mandates meticulous preparation with zeroing of aortic pressure, equalizing the guide catheter pressure with wire pressures before seating the guide catheter in the LMCA. However, since the guiding catheter could obstruct flow, particularly for ostial LMCA lesions, it should be disengaged once hyperaemia is induced, to reduce the effects of 'pressure-damping'. If this is proving difficult then the use of an additional wire within the other branch can be used to stabilize the distance from the catheter to the LMCA ostium.

For induction of hyperaemia, a continuous intravenous infusion is recommended, because when unseating the guide catheter, adequate administration of adenosine to distal microvasculature can be otherwise challenging. In addition, as shall be detailed later, the use of intravenous adenosine also enables an FFR pullback manouvre. Intravenous adenosine infusions, particularly when entering from the peripheral venous circulation, can produce several patterns of $\mathrm{Pd} / \mathrm{Pa}$ responses ${ }^{47}$. In order to overcome such variability, Johnson et al identified a stable period during adenosineinduced hyperaemia, called the "smart-minimum" (lowest '5 cardiac-cycle' average), that was found to be most reproducible ${ }^{47}$. Furthermore, it is important to remember that patients with 
LMCA disease often have contractile impairment which can result in elevated central venous and right atrial pressures. Since FFR assumes venous pressure is negligible, if measured venous pressures are significantly elevated, FFR values need to be interpreted cautiously regardless of the vessel being interrogated ${ }^{48}$.

\section{'Isolated' Left Main stenosis}

In the scenario of isolated LMCA disease (or 'LMCA equivalent' with contiguous downstream disease), there is no theoretical difference in measuring LMCA FFR when the wire is placed in either branch. FFR pullback manoeuvres should however be performed from both the distal LAD and LCx (see figure 5) because the physiological significance of downstream disease shouldn't be presumed from visual estimation alone ${ }^{1,9}$

\section{Left Main stenosis and epicardial disease in one downstream branch}

When concomitant to a LMCA stenosis, a significant stenosis is present in only one downstream branch, measuring the true FFR is still potentially confounded. As described earlier, the practice of measuring LMCA FFR by using a disease-free daughter vessel (figure 5) has been shown to be a relatively reliable with only minimal interference from downstream disease in the diseasedbranch (unless this is severe: $>90 \%$ diameter stenosis or FFR $<0.45$ ) $44-46$.

\section{Left main stenosis and disease in all downstream epicardial branches}

In the absence of a disease-free branch, serial lesion interplay becomes particularly challenging with a significant likelihood that distal stenoses can alter flow, and in turn the pressure gradient, across the $\mathrm{LMCA}^{30}$. At present, in patients having LMCA PCI, the practice of treating the greatest $\Delta$ FFR following manual FFR pullback appears to be the best available method and has been shown to be safe and reduce unnecessary revascularisation with acceptable outcomes at a mean followup period of 9 months ${ }^{33}$. We therefore recommend that for any LMCA stenosis, the pressure wire should be advanced distally in both epicardial branches to determine both FFR values. Following 
this, a fixed rate pressure wire pullback should be performed in both branches so as to allow estimation of physiological significance and length of all lesions. Recommendations following these pullback manoeuvres are summarised in Figure 5.6. Information from this physiological assessment should lead to all revascularisation strategies being considered, not just PCI; particularly in the context of Diabetes or high Syntax Score and with the help of a Heart Team.

\subsubsection{Imaging LMCA disease as a surrogate of physiology?}

\section{Intravascular Ultrasound (IVUS)}

IVUS-guided-PCI has been associated with better outcomes, due to improved vessel sizing for optimal stent deployment ${ }^{49}$. It is therefore attractive to also use intravascular imaging as a diagnostic tool if sufficient correlation to physiological measures can be found. As such, several studies have assessed the use of Minimum Luminal Area (MLA), derived from IVUS values, for inferring the functional significance of LMCA disease. Jasti et al were the first to show this correlation in a study of 55 patients $^{50}$. Perhaps due to the smaller perceived variation in LMCA length, diameter and eccentricity, there is better concordance between IVUS and FFR in the LMCA compared to other vessels ${ }^{51}$. Subsequently, other studies have demonstrated the relationship between the degree of MLA reduction and subsequent MACE rates, with the multicenter LITRO study showing that an IVUS-MLA of $6 \mathrm{~mm}^{2}$ could be used for safely deferring LMCA revascularisation, yielding acceptable long-term clinical results ${ }^{52}$. Despite such data, there remains significant variability regarding which IVUS cut-off values correlate best with FFR.

Apart from the impact of demographics on the normal reference range, this significant variation in IVUS MLA values can be explained physiologically, because the pressure drop across a narrowing is governed by several other factors in addition to the MLA (Figure 2). Nonetheless, the literature does suggest that using IVUS thresholds in the large range of $4.5-7.5 \mathrm{~mm}^{2}$ provides some improvement to angiography alone in determining the functional significance of a LMCA 
stenosis, provided a true LMCA cross-sectional cut is obtained ${ }^{53,54}$. It is important to keep in mind that the studies supporting using thresholds as low as $4.5 \mathrm{~mm}^{2}$ were conducted in populations with only Asian patients, who may have relatively small hearts, with the authors of the study recognizing that the global applicability of the results maybe limited ${ }^{53}$.

\section{Optical Coherence Tomography (OCT)}

OCT offers greater spatial resolution at the cost tissue penetration, and thus potentially clearer delineation of the intima-luminal limit, with data showing more accurate and reproducible assessment of lesion diameter when compared to IVUS using phantom stenosis models ${ }^{55}$. Whilst there is no data correlating OCT-derived LMCA luminal dimensions with FFR, there is suggestion from a study by Gonzalo et a ${ }^{56}$ that OCT is moderately efficacious versus IVUS in identifying hemodynamically severe coronary stenoses in smaller epicardial vessels with MLA $<3 \mathrm{~mm}^{2}$. However, as with other studies attempting to use intravascular imaging as a surrogate for physiology, they found specificity levels were too low to use as a substitute to FFR for functional assessment.

\subsubsection{Algorithm for Physiological Assessment of LMCA Disease Incorporating the Disease Free Side-branch}

Based on the evidence described in 5.5.3, when LMCA disease is present alongside downstream serial disease, the following algorithm can be utilised for physiological assessment in this unique case of serial disease (Figure 5.6). 

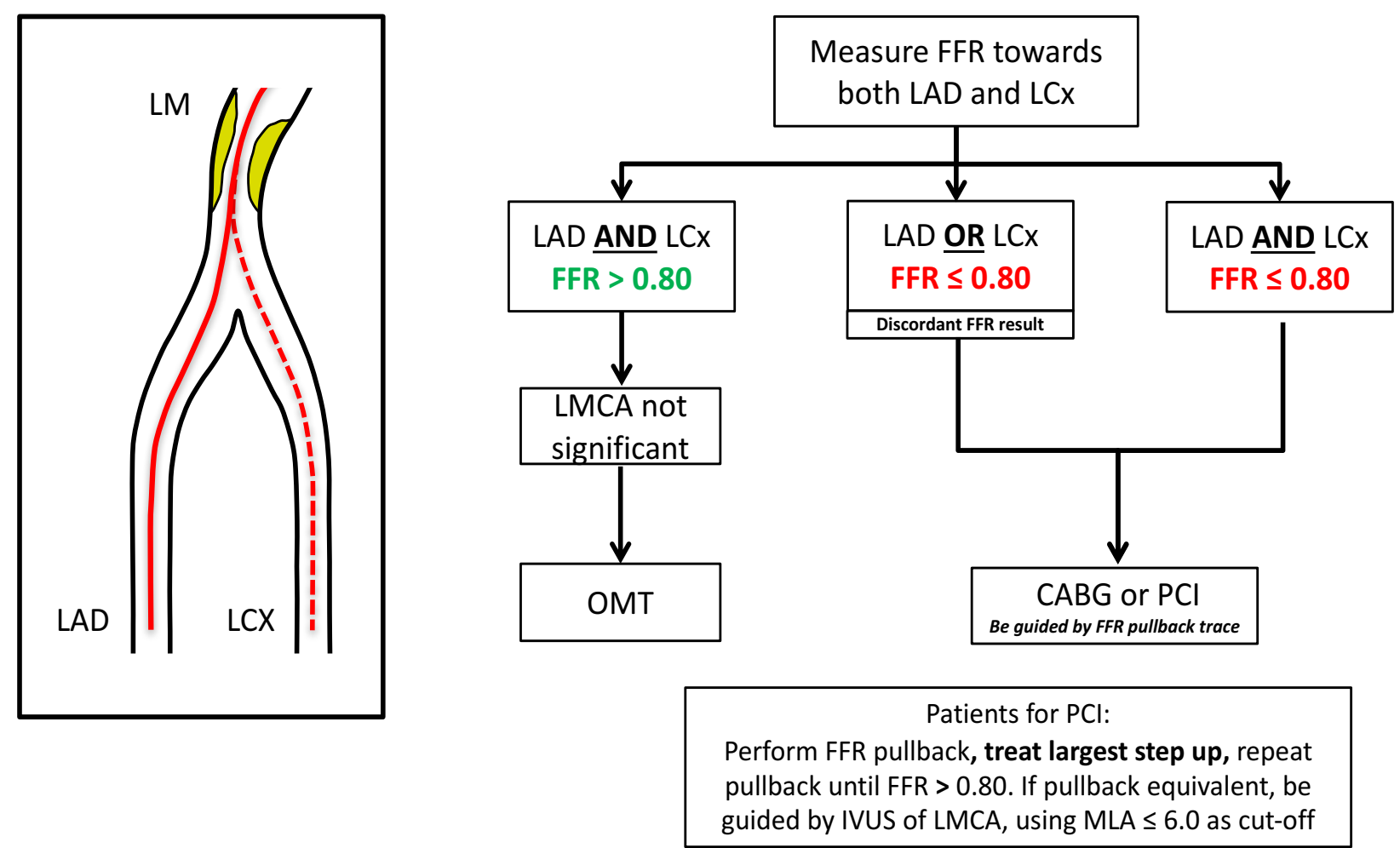

Figure 5.6: A suggested decision-making algorithm when confronted with LMCA disease, taking into account various combinations of downstream disease

As evident in this algorithm, there is still significant uncertainty in physiologically assessing LMCA disease when there is downstream serial disease. At present, the best available recommendation is to treat largest step-up and re-measure, despite the potential flaws of this method (illustrated in figure 5.3 and 5.4).

\subsection{NON-INVASIVE FFR BY COMPUTED TOMOGRAPHY (FFR 6 T)}

\subsubsection{An Introduction to FFR CT}

Coronary computed tomographic angiography (CTA) is now established as a clinically valuable non-invasive anatomical test for the detection and exclusion of significant coronary disease with a number of multicentre trials showing coronary CTA to be an ideal test for the exclusion and detection of coronary disease using invasive angiography as the reference ${ }^{57,58}$. Despite this, owing 
to its relatively low positive predictive value of $48 \%$ and inability to determine functional significance $^{58}$, its use has generally (but not exclusively ${ }^{59}$ ) been restricted to patients with chest pain at a low-intermediate risk of having coronary artery disease (CAD ${ }^{60}$.

For patients at higher risk and those found to have significant CAD during CTA, patients often proceed to invasive coronary angiography. For these patients undergoing invasive coronary angiography, it has been shown that over a half are found to have no significant disease ${ }^{61}$ and even once significant CAD is detected on invasive angiography, there is growing evidence that the benefit of revascularisation is only derived from targeting myocardial ischaemia ${ }^{7}$, with further evidence awaited from the ongoing ISCHEMIA trial (NCT01471522).

As already discussed in section 5.1, we are in an era of ischaemia guided revascularisation with FFR representing the gold standard of physiological assessment of CAD at the time of diagnostic angiography. In recent years, it has now become possible to estimate FFR from standard coronary CTA datasets. This technique has been developed by the company HeartFlow (Redwood City,

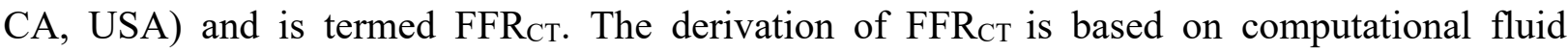
dynamics (CFD) which involves applied mathematics, physics and computational software to visualize how fluids interact with adjacent objects. Computational fluid dynamics relies on the Navier-Stokes equations which describe how the velocity, pressure, temperate and density of a moving fluid are related. The scientific principles of Computational Fluid Dynamics behind the calculation of FFR $\mathrm{C}_{\mathrm{CT}}$ and have been extensively published ${ }^{62,63}$ and are beyond the scope of the current manuscript. A step by step approach to the process is summarized in figure 5.7. 


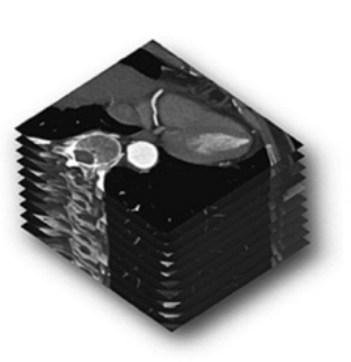

3-D FFR ${ }_{\text {CT }}$ solution

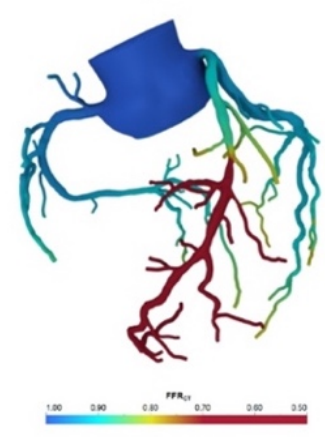

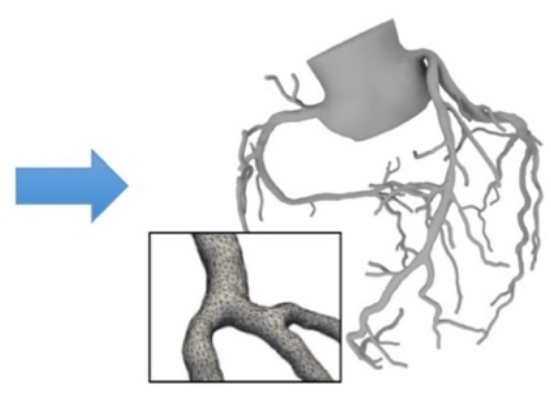

Computation of coronary flow

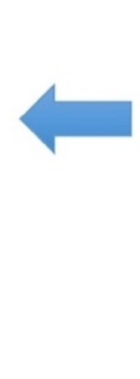

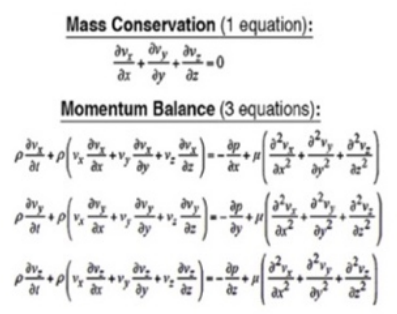
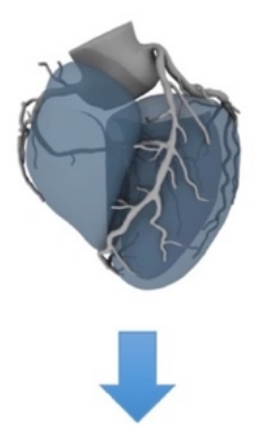

Modeling maximal hyperaemia

Figure 5.7: Method of FFR $\mathrm{CT}_{\mathrm{C}}$ Computation: 1. Coronary $\mathrm{CT}$ angiography images acquired using standard protocols. 2. Anatomical model enabling construction of a tetrahedral mesh resulting in millions of discrete points for computation of coronary pressure and flow, including branch points. 3. Physiological model of coronary circulation created with specified inflow and outflow boundary conditions with resting coronary flow is based on myocardial mass. 4. Modelling of maximal hyperaemia to reflect expected reduction in peripheral resistance resulting from adenosine administration. 5. Numerical solution of Navier-Stokes equations that govern the fluid dynamics of blood flow so that Fractional flow reserved is computed. 6. 3-D computation of $\mathrm{FFR}_{\mathrm{CT}}$ throughout the coronary artery tree under simulated maximal hyperaemia

\section{FFR $R_{C T}$ diagnostic accuracy}

Following on from the basic validation of the model ${ }^{64}$, a series of studies designed to test the diagnostic performance of FFR $_{\mathrm{CT}}$ against invasive FFR have been performed including the Diagnosis of Ischaemia-Causing Stenoses obtained via NoninvasivE Fractional FLOW Reserve (DISCOVER-FLOW), The Determination of Fractional Flow Reserve by Anatomic Computed Tomographic Angiography (DeFACTO) study and Analysis of Coronary Blood Flow Using CT Angiography: Next Steps (NXT Trial) .

The DISCOVER-FLOW Trial was a study of 103 patients with suspected or known CAD who underwent coronary CTA, ICA and FFR at 4 sites ${ }^{65}$. Of the 156 vessels for which invasive FFR 
was performed, an $\mathrm{FFR}_{\mathrm{CT}} \leq 0.8$ was shown to have an accuracy of $84.3 \%$, sensitivity of $87.9 \%$, specificity of $82.2 \%$, a positive predictive value of $73.9 \%$ and a negative predictive value of 92.2\% against an invasive FFR measurement of $\leq 0.8$. This compared favourably to simple coronary CTA assessment of the stenoses which had a significantly lower accuracy of $58.6 \%$ a positive predictive accuracy of $48.6 \%$. Following this, the DeFACTO study ${ }^{66}$ was designed to evaluate the diagnostic performance of $\mathrm{FFR}_{\mathrm{CT}}$ against invasive FFR in stable patients with suspected native coronary artery disease who were scheduled for invasive coronary angiography within a 60-day timeframe. Of the 252 patients in 5 countries, 137 (54.5\%) were found to have an abnormal FFR determined by ICA. The diagnostic accuracy of FFR $\mathrm{CT}_{\mathrm{T}}$ and coronary CTA was $73 \%$ (95\% CI 67\%-78\%), which did not meet the primary endpoint of $70 \%$ of the lower bound of the 95\% confidence interval. Despite this, $\mathrm{FFR}_{\mathrm{CT}}$ showed superior discrimination against a coronary stenosis severity of $\geq 50 \%$ (AUC 0.81 vs. 0.68 ). When vessels interrogated by FFR were compared against $\mathrm{FFR}_{\mathrm{CT}}$ and coronary $\mathrm{CTA}$ alone, $\mathrm{FFR}_{\mathrm{CT}}$ showed superior diagnostic ability with sensitivity of $80 \%$ and specificity of $61 \%$. The third major trial evaluating the diagnostic performance of FFR $_{\mathrm{CT}}$ was the NXT Trial ${ }^{67}$. This evaluated the diagnostic accuracy of FFR $\mathrm{CT}_{\mathrm{CT}}$ in 251 patients, each with at least one luminal stenosis on coronary CTA of between $30-90 \%$ in a vessel $\geq 2 \mathrm{~mm}$. The diagnostic accuracy, sensitivity, specificity, PPV and NPV for FFR $\mathrm{CT}_{\mathrm{C}}$ was $86 \%, 84 \%, 86 \%, 61 \%$ and $95 \%$ respectively. A notable difference from the DeFACTO trial was that the NXT study used an improved iteration of the $\mathrm{FFR}_{\mathrm{CT}}$ algorithms and the cardiac CT acquisition protocols and invasive FFR measurement protocols were standardized across all sites, factors which were believed to have adversely impacted on the results of the DeFACTO study.

\section{Clinical utility and patient outcomes from $F F R_{C T}$}

Although the DISOVER FLOW, DeFACTO and NXT Trials suggested that FFR $\mathrm{CT}_{\mathrm{C}}$ had the potential ability to enhance the diagnostic capabilities of coronary CTA, its use as a clinical tool had not been formally evaluated. Curzen et al. therefore elected to study whether or not the routine availability of FFR $\mathrm{CT}_{\mathrm{T}}$ would change clinical decision compared to the results from coronary CTA 
alone $^{68}$. In this study, 3 interventional cardiologists assessed the CTA report in order to reach a consensus management strategy for 200 consecutive patients who had undergone coronary CTA in the NXT trial. The cardiologists were then shown the FFR $\mathrm{CT}_{\mathrm{T}}$ data for each case and again produced a consensus management of which vessels and lesions were significant. The prescribed CTA-derived management strategy was changed in $36 \%$ of cases, with a reduction in PCI rates seen in $30 \%$, and a change in the PCI target vessel treated in $18 \%$. These results were very similar to those derived from the original RIPCORD study, examining invasive angiography and FFR 9 .

The largest $\mathrm{FFR}_{\mathrm{CT}}$ study to date is PLATFORM, a prospective cohort study of 585 patients ${ }^{69}$. It was designed to test whether the routine evaluation of patients with suspected CAD by coronary CTA/FFR ${ }_{\mathrm{CT}}$ as their default test, would result in lower rates of ICA showing no obstructive disease, without any consequence in terms of major adverse cardiac event rate compared to routine clinical care. The study participants were enrolled in two consecutive cohorts assigned to receive standard care testing or $\mathrm{CTA} / \mathrm{FFR}_{\mathrm{CT}}$ testing. In the invasive cohort, $100 \%$ of patients underwent invasive coronary angiography in the pre-specified routine care invasive arm, with $73.3 \%$ showing no significant obstructive disease. By contrast, only $39.4 \%$ of patients allocated to CTA/FFR $\mathrm{CT}_{\mathrm{C}}$ required invasive coronary angiography based upon this test, of whom only $31.6 \%$ showed no obstructive disease. PLATFORM therefore showed that coronary CTA/ FFR $\mathrm{CT}_{\mathrm{CT}}$ has the ability to reduce the number of stable patients who require invasive coronary angiography that shows no significant coronary disease at invasive angiography. The other important result from PLATFORM was that at 1 year no MACE events had occurred in the 117 patients whose coronary angiograms were deemed unnecessary based on reassuring coronary CTA/FFR $\mathrm{CT}_{\text {findings }}{ }^{70}$.

\subsubsection{A Role in Serial Disease?}

In cases where $\mathrm{CT}$ images are good, it may be possible to make a thorough assessment of individual stenosis geometry, and therefore an accurate estimation of the frictional and separation coefficient of a stenosis (Figure 5.2). Modelling flow and pressure changes from this precise anatomical knowledge lends itself to assessing true stenosis significance, independent of 
disease and flow conditions elsewhere in the vessel. As discussed, FFR $\mathrm{CT}_{\mathrm{T}}$ has largely been developed and validated in vessels with single/discrete stenoses, but these inherent advantages of $\mathrm{CT}$ and the underpinning $\mathrm{CFD}$ theory make $\mathrm{FFR}_{\mathrm{CT}}-$ related innovations potentially an ideal tool for unpicking serial stenosis physiology before a guidewire has entered the body.

A small sub-study of the NXT trial, looking at 18 vessels with serial stenoses compared translesional FFR CT $_{\text {gradients against trans-lesional FFR values after manual pullback }}{ }^{71}$. Whilst this showed a degree of correlation, the invasive FFR values, against which trans-lesional FFR $\mathrm{RT}_{\mathrm{CT}}$ values were compared, are themselves substantially flawed. A validation of trans-lesional FFR $\mathrm{CT}_{\mathrm{C}}$ values against the true invasive trans-lesional FFR values (with the lesion present in isolation) is yet to be done. Once this step is realised (within this thesis), the iterative and machine learning processes built into the underlying technology may make a future $\mathrm{FFR}_{\mathrm{CT}}$ solution to serial disease a possibility.

\subsection{AIMS \& OBJECTIVES}

The broad aim of this thesis is to understand and optimise how to physiologically assess the significance of individual stenoses in serial/diffuse coronary artery disease. In order to achieve this aim, this thesis will address the following objectives:

1) Improve our understanding of the nature of serial stenosis interplay and the factors that influence this using a 3-D printed model of serial CAD ('Error' when measuring a biological variable can be defined as an unbiased assessment of the difference against the normal biological variability of the index and therefore the existence of this potential error needs to be objectively assessed). Once established, I aim to develop and test an algorithm to correct for 
serial stenosis interplay when using the commonest invasive physiological tool of FFR (Experimental Chapter 6)

2) Compare various commonly used invasive physiological indices in assessing serial CAD and understand serial stenoses further using simultaneous measurements of Doppler flow and pressure. Following this we can truly understand the value of the correction equation created (Experimental Chapter 7)

3) Assess the value of both conventional FFR $\mathrm{CT}_{\mathrm{CT}}$ and a novel FFR $\mathrm{FT}_{\mathrm{CT}}$-derived PCI Planning tool in serial CAD. Estimating Fractional Flow Reserve from Cardiac CT (FFR $\mathrm{FT})$ is growing in popularity with growing evidence to support its use as an adjunct to conventional assessments. This thesis provides us with an opportunity to explore whether the underpinning CFD principles behind $\mathrm{FFR}_{\mathrm{CT}}$ can help overcome the complexities in assessing serial CAD (Experimental Chapter 8)

After addressing these 3 objectives in the respective experimental chapters, we will be in a position to begin assessing the clinical utility of any novel solutions developed (Experimental Chapter 9) and also suggest future directions of research (Synthesis, chapter 10) for this commonly-encountered, but as yet unresolved, clinical conundrum. 


\section{EXPERIMENTAL CHAPTER: IN VITRO ASSESSMENT OF SERIAL CORONARY ARTERY DISEASE}

Presented at ESC 2017 (Barcelona) and AHA 2017 (Anaheim)

Published in the Journal of the American Heart Association

\section{Acknowledgements}

I would like to pay particular gratitude to the following people for their help with this section:

Dr Nicholas Gaddum (Bioengineering Dept, KCL)

Dr Jack Lee (Bioengineering Dept, KCL)

Dr Matthew Ryan (BHF Clinical Research Fellow)

Ms. Anjalee Chattersingh (MSc Student)

Dr Kseniia Erusalnova (MSc Student)

Prof Phil Chowienczyk (Secondary Supervisor)

Prof Divaka Perera (Primary Supervisor) 


\section{Introduction}

Growing evidence supports ischaemia-guided revascularisation for coronary artery disease $(\mathrm{CAD})^{7}$. Ischaemia assessment has now evolved from assessment of myocardial territories using non-invasive imaging to being able to assess physiological disease in specific vessels, at the time of diagnostic angiography. This involves calculating the ratio of distal coronary pressure to aortic pressure at maximal hyperaemia (Fractional Flow Reserve, FFR $)^{8}$, at rest during the entire cardiac cycle $(\mathrm{Pd} / \mathrm{Pa})^{72}$ or at rest during a defined-phase of diastole (Instantaneous Wave-Free Ratio, iFR $)^{73}$. Physiology-guided revascularisation appears to confer significant clinical and prognostic benefit over management based on angiographic appearance alone $e^{4,10,74}$.

Whilst these pressure-derived indices are well established and validated for vessels with single lesions, given the systemic nature of atherosclerosis, coronary stenoses are often found in tandem with other focal or diffusely diseased segments within the same vessel. In such instances, the decision to revascularise and the mode of revascularisation chosen rely on identifying the true individual contribution of lesions and not just the cumulative impact of disease in the entire vessel. However, each diseased segment affects the fluid dynamics of the other, which makes it difficult to apply conventional physiological techniques to identify the true significance of a lesion within a serially diseased vessel ${ }^{27,75}$. Knowing the true functional significance of each stenosis enables the appropriate stenosis to be targeted and helps ensure the correct revascularisation strategy is chosen, whether this is PCI, CABG or medical therapy.

Theoretical solutions have been developed that involve complex formulae ${ }^{28}$ using measurements of intracoronary pressure at various points in the artery and determination of coronary wedge pressure, which in turn is acquired during transient balloon occlusion of the vesse ${ }^{27,30}$. The rationale for incorporating a wedge pressure measurement was to standardise and account for variability in collateral flow across a stenosis ${ }^{30}$. A major drawback of this technique, which has limited the adoption of these formulae in clinical practice, is that in order to obtain a wedge 
pressure measurement, it is necessary to balloon occlude the coronary artery, which carries a risk of dissection. This could have clinical sequelae if angiographically undetectable at the time, or if detected, may mandate stenting regardless of the results of physiological assessment. Other solutions to this problem have relied on the identification of a large disease-free side branch ${ }^{44,46}$ to separate the true significance of a proximal stenosis, although this scenario is only applicable to Left Main Coronary Artery (LMCA) disease. Furthermore, the relative accuracy of hyperaemic versus resting indices, such as $\mathrm{iFR}^{35}$, in the context of serial disease is unknown.

The aim of this study was firstly to develop an in vitro model based on 3D-printed configurations of tandem disease that is a realistic representation of pulsatile coronary circulations in hyperaemic conditions. Using these results, we aimed to characterise the factors that influence serial stenosis haemodynamic interplay and incorporate them in theoretical models to improve prediction of the true FFR of individual stenoses, without the need for wedge pressure measurements or angiographic guesses of stenosis and vessel diameters. We then sought to test such a solution within a clinical cohort of patents with serial CAD.

\section{Methods}

\section{Creating a Model of Tandem Coronary Artery Disease}

To model tandem disease and accurately control for variable stenosis geometry and the many factors that affect the pressure drop across a stenosis (figure 6.1), phantom tubes were created by 3-D printing (Objet 500 Connex3'TM, $600 \times 600 \times 1600 d p i)$ from the biocompatible 'TangoPlus FullCure PolyJet ${ }^{\circledR}$ ' photopolymer material (Objet ltd, Israel) that has been shown to adequately model arterial compliance ${ }^{76}$ (see figure 6.1). The tubes created were $150 \mathrm{~mm}$ long, with an internal diameter of $5 \mathrm{~mm}$ and wall thickness of $2 \mathrm{~mm}$ to simulate the dimensions and compliance of a typical Left Anterior Descending (LAD) artery. 


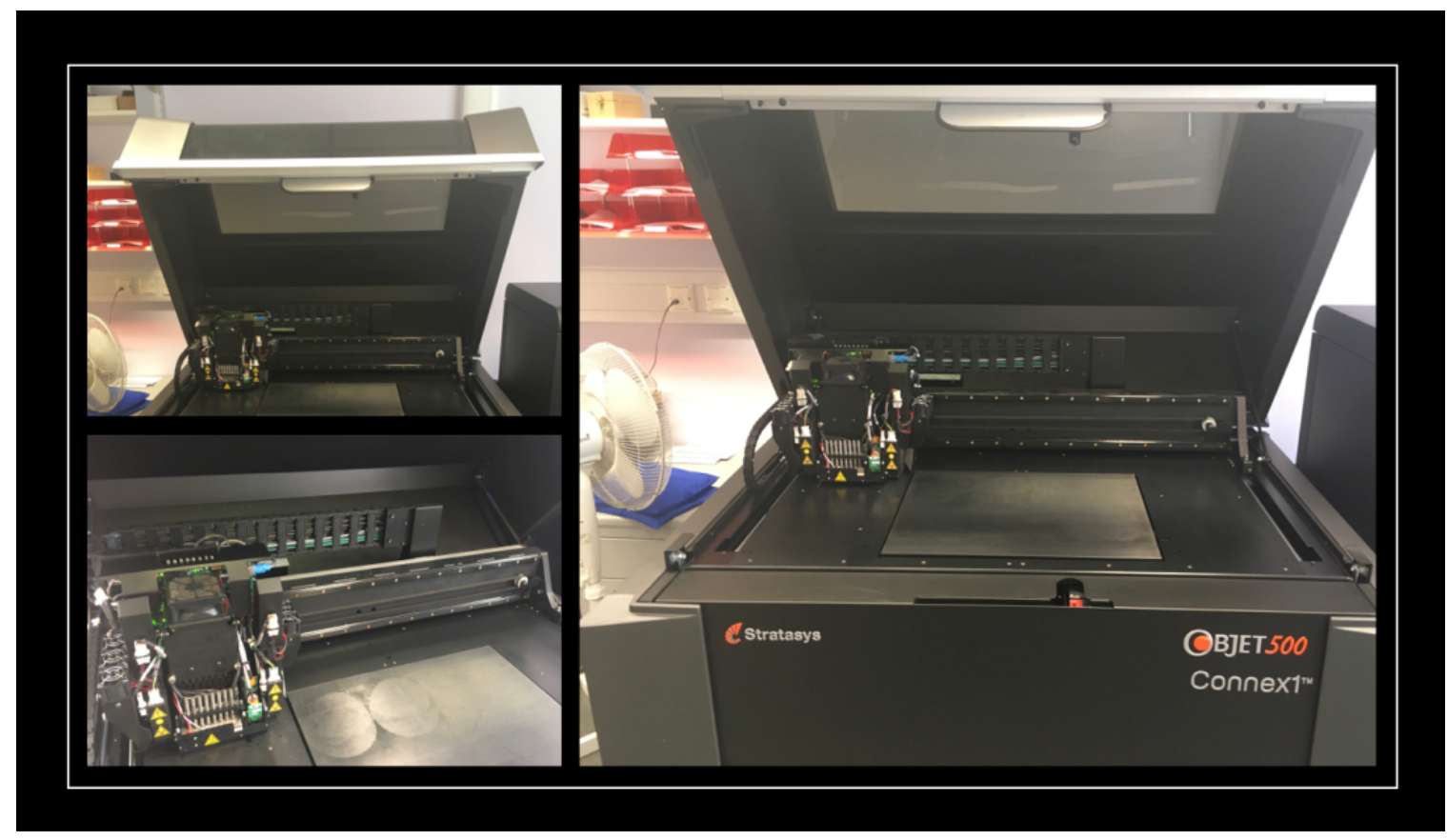

A

Marking to show the position and percentage severity of stenosis

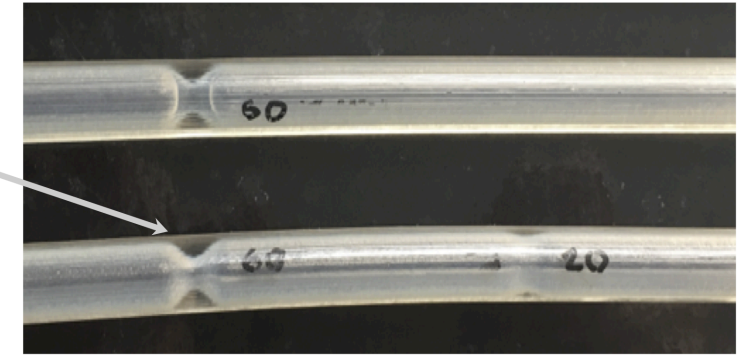

B
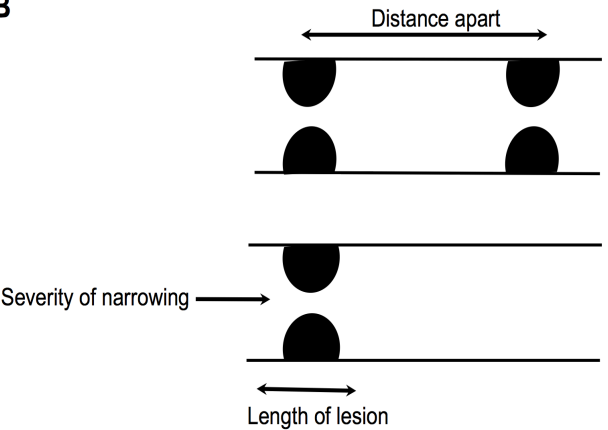

Figure 6.1: Top: Photograph of Objet 500 3-D printer used for creation of coronary phantoms. Bottom Part A: Photographs of a tube modelling two serial lesions and a tube with the corresponding single stenosis; number representing \% diameter stenosis. Part B: Schematic representation of these stenosis, showing the variables that were adjusted by 3 -D printing

A continuous flow model of coronary circulation was created to facilitate accurate measurement of pressure changes (figure 6.2). Continuous inlet pressure was created by using an electricallydriven water pump and capacitance chamber that connected to an aorta modelled from Polyjet tubing, with aortic pressure kept fixed. Polyjet tubing, modelling the LMCA, then branched off 
the aorta with the 3-D printed phantom attached, ensuring any connections themselves did not pose additional resistance (figure 6.2). A fixed length low compliance silicon tubing was added downstream of the phantom tube and calibrated to create similar resistance to the coronary microcirculation during adenosine induced hyperaemia. Distilled water was used as the fluid for all experiments, in line with previous in vitro models of coronary circulation ${ }^{41,77}$ that presume Newtonian conditions are maintained within coronary arteries under physiological conditions ${ }^{78}$. The use of 3D-printing enabled all stenoses to have a fixed geometry to reduce variability in other factors influencing the pressure drop across a stenosis ${ }^{23}$.

\section{Measurement of FFR}

Using this set up, a pressure wire (Phillips Volcano ${ }^{\mathrm{TM}}$ ) was normalised to aortic pressure and advanced distal to the serial lesions. FFR was calculated as the ratio of distal coronary pressure to aortic pressure, with conditions presumed equivalent to hyperaemia given the continuous flow setup with fixed and minimal distal resistance. The pressure-wire was pulled back through the lesions at $1 \mathrm{~mm} / \mathrm{s}$ using a modified pullback device (Philips Volcano ${ }^{\mathrm{TM}} \mathrm{R} 100$ ) to generate FFR pullback curves with all experiments repeated in triplicate; mean values from these are quoted throughout (figure 6.2). Each 3D-printed configuration of tandem disease (varying in length of stenoses, separation and severity) was then replaced with the corresponding 3D-printed tube containing the isolated stenosis to simulate treatment with coronary angioplasty (PCI) (figure 6.2). 


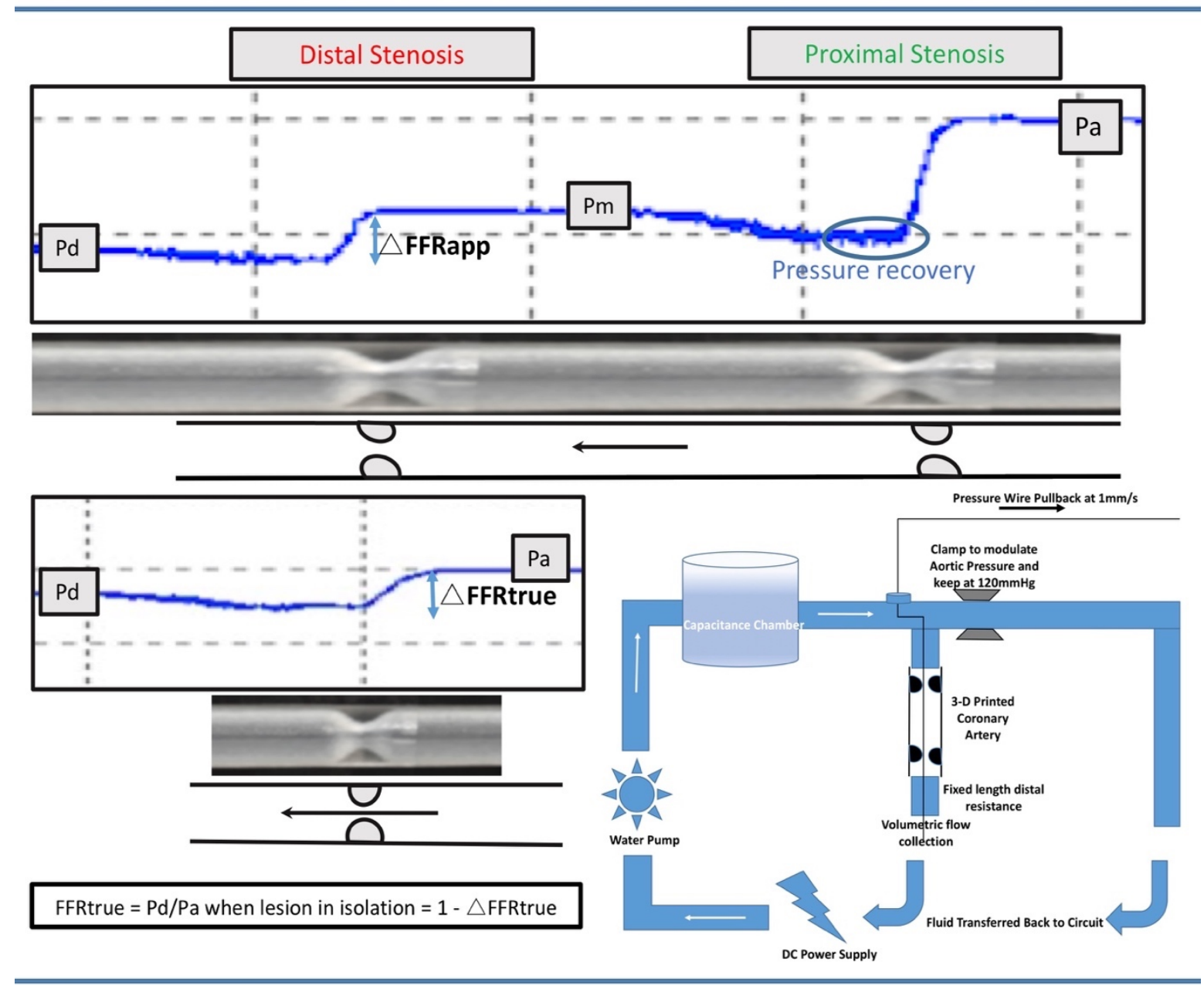

Figure 6.2: How 3-D printing Was Used to Model Pressure-Wire Measurements in Serial Disease. Top: Photographs of a 3D printed tube modelling tandem lesions with a pressure wire pullback demonstrating distal coronary pressure $(\mathrm{Pd})$, pressure between lesions $(\mathrm{Pm})$ and aortic pressure $(\mathrm{Pa}) . \triangle \mathrm{FFR}_{\text {app }}$ represents the apparent pressure gradient across a stenosis when in the presence of another. Also demonstrated is the phenomenon of pressure recovery after a tight stenosis - showing further evidence of our model replicating in vivo physiology. Bottom Left: $3 \mathrm{D}$ printed tube with the corresponding single stenosis in isolation. $\Delta \mathrm{FFR}_{\text {true }}$ represents the true pressure gradient across a stenosis. Bottom Right: Diagrammatic Representation of Continuous

Flow Phantom model used to model serial stenosis haemodynamic interplay.

\section{Validation of in vitro model of Serial CAD within Coronary Circulation}

To ensure our wet laboratory model of coronary circulation was a valid representation of in vivo pulsatile coronary physiology, we carried out the following tests of the model before commencing the experiments:

\section{Test-Retest Variability}

The first test to ensure clinical validity is to ensure there is high test-retest reliability, as seen with in vivo FFR measurements ${ }^{79}$. Triplicate pressure gradient measurements were made for 
each stenosis created, allowing calculation of intra-class correlation to ensure high test-retest reliability. The results of this test revealed a strong intra-class correlation of $0.973(0.95-0.98)$ for average measures, suggesting good test-retest reliability.

\section{Compliance Testing}

To ensure the 3-D printed tubes could represent coronary vessels, they have to show similar compliance to in vivo coronary arteries. To test this, we created pressure-volume loops in a blank tube made of the same material (see figure 6.3). The results of this test revealed hysteresis that compared favourably to published data on coronary artery compliance ${ }^{76}$.

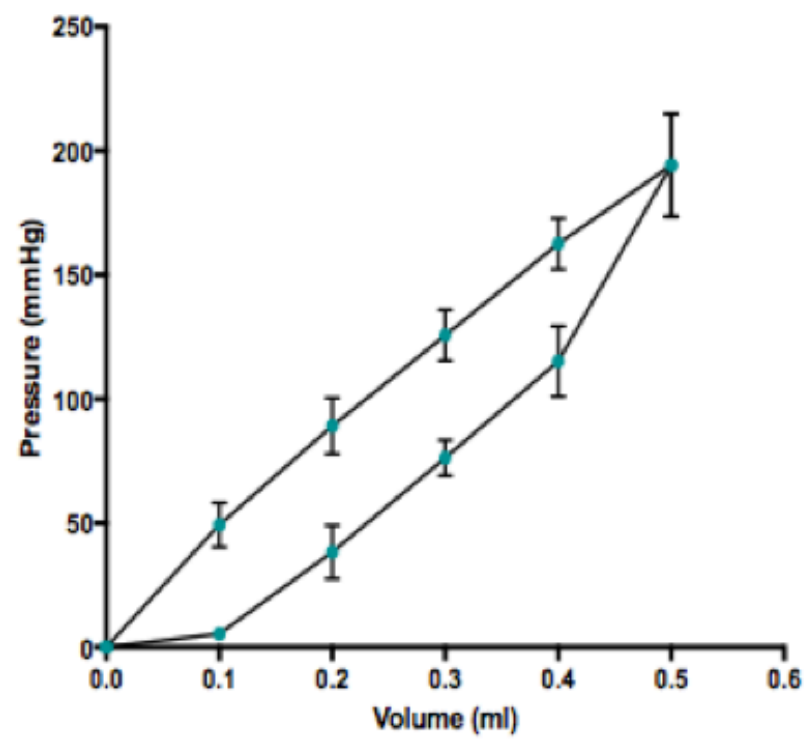

Figure 6.3: Graph to demonstrate the pressure-volume loop created during compliance testing of the coronary phantom material. Data represent mean \pm standard deviation.

\section{Comparison of Continuous Flow versus Pulsatile Flow Model}

Prior to my work within our wet laboratory, a pulsatile in silico model was created and validated in a separate study to assess the contribution of the ventricle to peripheral arterial pressure ${ }^{80}$. In our study, measurements were made using a continuous flow model (figure 6.2) as they were easier to obtain. Before doing so, however, we made a comparison of pressure measurements across a range of stenoses from our continuous flow model against measurements from this previously validated pulsatile flow mode ${ }^{80}$ (Figure 6.4), with results demonstrating a strong correlation. 


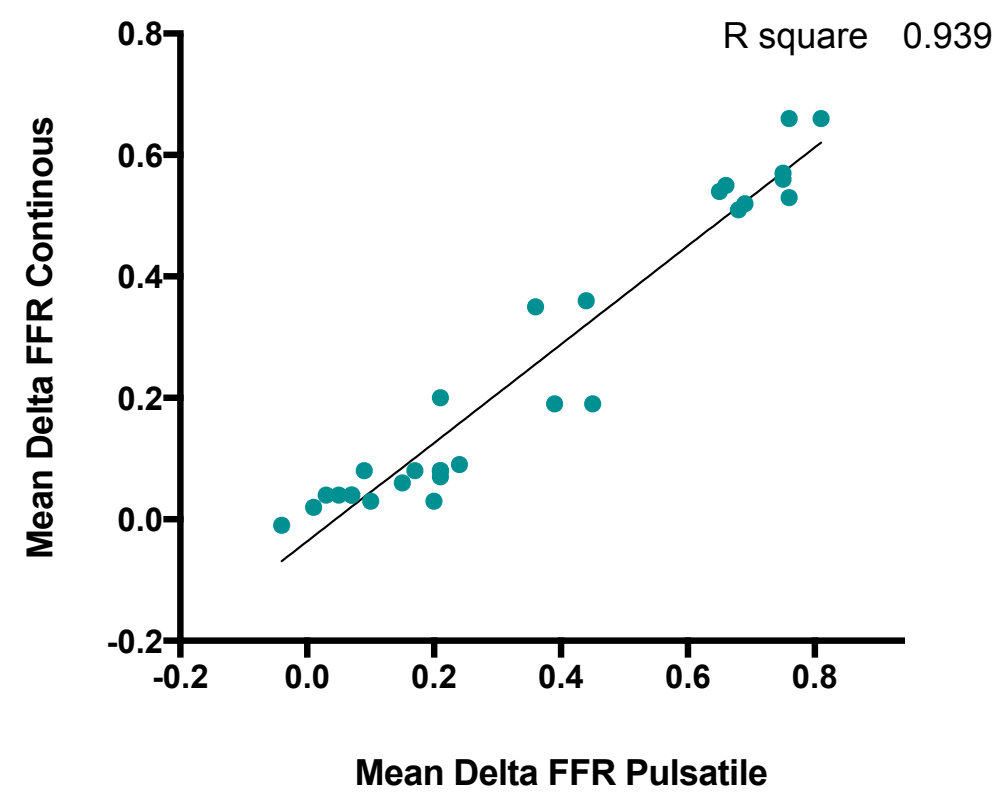

Figure 6.4: Scatterplot with Linear Regression Analysis demonstrating a strong correlation between $\triangle$ FFR values obtained in the pulsatile flow model and the continuous flow model $(\mathrm{P}<$ $0.001 ; n=29)$.

Assessing Effect of Stenosis Severity (Luminal Narrowing) on Trans-stenotic Pressure Drop The effect of luminal area reduction on the pressure drop across each stenosis was assessed and compared with previously reported coronary physiological data of pressure drops across stenoses in hyperaemic conditions ${ }^{23}$ (Figure 6.5). This revealed an exponential relationship between luminal narrowing and the pressure drop across a stenosis: similar to the relationship that has previously been demonstrated in vivo ${ }^{23,34}$.

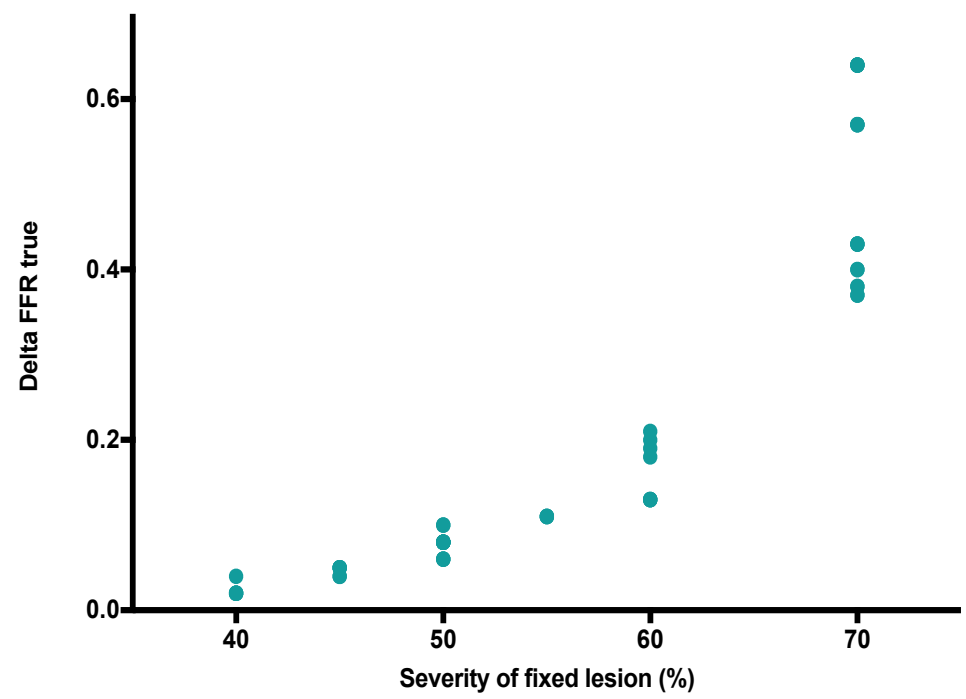

Figure 6.5: Scatterplot of the effect of lesion severity on $\Delta$ FFRtrue (mean values). 
Using pressure gradients as an analogue to blood flow impairment is only possible if the relationship between pressure and flow across a stenosis is similarly curvi-linear to that seen in vivo. Seminal experiments by Gould et $\mathrm{a}^{23}$ in canines showed pressure gradients and flow velocity are related non-linearly (figure 6.6, part A). In order to show our model replicated this important physiological trait, we sought to measure flow rate impairment in a cohort of 3-D printed serial stenosis phantoms. We did this by measuring volumetric flow rate of water at the distal end of phantoms with stenoses and subtracting from flow rate at the distal end of a phantom with no stenosis. By plotting flow rate impairment (analogous to flow velocity) against pressure gradient, we were able to demonstrate that the curvilinear relationship between pressure and flow is replicated (Figure 6.6 Part B).

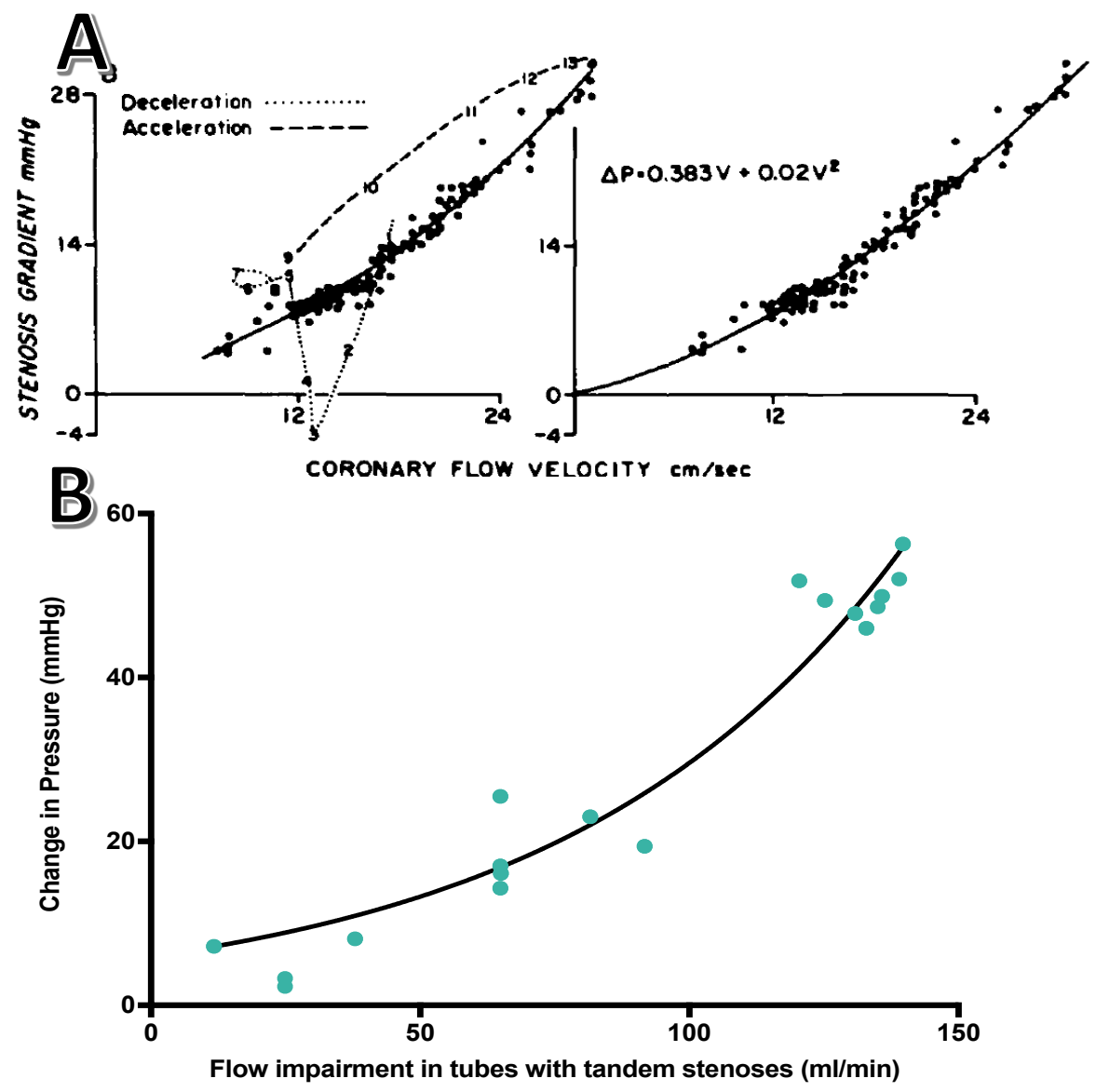

Figure 6.6: Part A: Representation of relationship between the stenosis gradient and coronary flow velocity from the Gould et al experiments of pressure-flow characteristics of coronary 
stenoses in un-sedated dogs at rest and during coronary vasodilatation $\left.{ }^{23}\right)$. Part B: Scatterplot of the change in pressure across each validation phantom versus the flow impairment it caused.

Curvilinear trendline shown to similarity with seminal experiments by Gould et al (part A).

\section{Pressure Recovery}

Blood in a coronary artery behaves as a Newtonian fluid, much like water, as already discussed ${ }^{81}$. Further evidence of this was incidentally found by discovering the effect of pressure recovery (due to the Bernoulli Effect) just distal to severe stenoses, demonstrated in example pullback trace in figure 6.2). This phenomenon often exists in vivo, just distal to severe concentric stenoses (e.g. Severe Aortic Stenosis). Where any pressure recovery was observed (Figure 6.2), $\mathrm{P}_{\mathrm{d}}$ immediately proximal to the distal lesion, prior to pressure recovery phase, was taken as the inter-stenotic pressure to calculate $\Delta \mathrm{FFR}_{\text {app }}$ and $\Delta \mathrm{FFR}$ true.

\section{Limitations of the in vitro Coronary Circulation Model}

In an idealised model, we would also want to include phantoms that modelled bifurcations, collateral circulation, variable myocardial mass etc. These are complexities to be added in later iterations once we establish which factors are most important in serial stenosis interplay.

\section{Measuring FFRapp and FFRtrue using the In Vitro Model}

For a given stenosis, the apparent $\mathrm{Pd} / \mathrm{Pa}$ gradient $(\Delta \mathrm{FFR}$ app $)$ is the trans-stenotic pressure gradient across a given lesion when the measurement was made in the presence of both lesions, whereas the actual trans-stenotic pressure gradient $(\Delta \mathrm{FFR}$ true $)$ is when that stenosis is present in isolation, with the accompanying stenosis removed (figure 6.2). The extent of serial stenosis haemodynamic interplay was assessed by examining the magnitude of difference between $\Delta \mathrm{FFR}_{\mathrm{app}}$ and $\Delta \mathrm{FFR}_{\text {true, }}$ with various combinations of serial disease. The impact of the following on this difference were assessed:

i) Total FFR (a function of distal pressure, Pd)

ii) Average severity ( $\%$ diameter reduction) of both stenoses 
iii) Length of stenoses (both physical length from 3D-printed geometries and physiological length from pullback traces performed at $1 \mathrm{~mm} / \mathrm{s}$ )

iv) Separation distance between the serial stenoses (in $\mathrm{mm}$ )

v) Position of the stenosis in question (i.e. proximal or distal to the accompanying stenosis).

The existence of pressure recovery was only seen in severe stenoses ( $>70 \%$ diameter stenosis) and therefore the impact of the magnitude of pressure recovery (as we do when assessing valvular stenoses with echocardiography) was not assessed.

\section{Statistical Analysis}

Data were analysed using SPSS Statistics Version 22 and Graph Pad Prism Version 7.0a for Mac OS X. Data are expressed as mean \pm SD unless stated otherwise. Paired t tests were used to compare continuous variables (the effect on the degree of stenosis underestimation) after normality of data was visually assessed using histograms and Q-Q plots. For all statistical analysis, $\mathrm{P}<0.05$ is considered statistically significant. An empirical correction equation was developed using stepwise simple linear regression modelling. Details of the theoretical correction equation are stated in the appendix to this chapter. The incremental value of the correction equation compared to $\Delta \mathrm{FFR}_{\mathrm{app}}$ from a conventional pressure-wire pullback was determined by the proportion of cases where an incorrectly classified lesion (according to FFR threshold of 0.8 ) was correctly reclassified.

\section{Results}

\section{In Vitro Model Validation}

Measurements of trans-lesional pressure drop in the continuous flow model showed high testretest reliability (intra-class correlation of three measurements for each lesion $0.97, \mathrm{P}<0.05$ ). The model demonstrated a non-linear relationship between stenosis severity (quantified by diameter stenosis) and pressure gradient. In addition, The compliance and hysteresis loops generated by 
varying pressure and flow were similar to those reported in vivo ${ }^{82}$. The model also showed strong statistical agreeability $(\mathrm{r}=0.94, \mathrm{p}<0.05)$ with measurements made in the previously validated pulsatile model ${ }^{80}$. Furthermore, using volumetric flow-rate measurements distal to the 3 -D printed phantoms, we were able to replicate accepted pressure drop vs. flow velocity relationships ${ }^{23}$. Validation data is summarised in the supplementary appendix. In addition, as described in Figure 6.2 , the concentric stenosis geometry meant that for severe lesions the phenomenon of "pressure recovery' was often observed due to the Bernoulli effect. Where any pressure recovery was observed, $\mathrm{P}_{\mathrm{d}}$ immediately proximal to the distal lesion, prior to pressure recovery phase, was taken as the inter-stenotic pressure to calculate $\Delta \mathrm{FFR}_{\text {app }}$ and $\Delta \mathrm{FFR}_{\text {true. }}$

Overview of Results

In total, 52 combinations of tandem lesions were analysed in vitro with average stenosis diameter stenosis of $55 \%$ and overall vessel FFR of $0.66+/-0.23$. There was a significant difference between $\Delta \mathrm{FFR}_{\mathrm{app}}$ and $\Delta \mathrm{FFR}$ true, with the true contribution of a given lesion being underestimated in the presence of an additional lesion $85 \%$ of cases. The overall difference between the $\mathrm{FFR}_{\text {app }}$ and $\mathrm{FFR}_{\text {true }}$ was $17.1 \%$ as a proportion of $\Delta \mathrm{FFR}$ true (absolute difference $0.036+/-0.048)$ with increasing lesion underestimation and variance between $F_{F R}$ app and FFR true $_{\text {tro }}$ with increasing total burden of disease (represented by Total FFR), see figure 6.7. The degree of this underestimation was similar $(\mathrm{P}>0.05)$, regardless of whether the distal stenosis or proximal stenosis was removed.

The 3D-printed model also enabled assessment of other factors on the extent of serial stenosis interplay including Lesion length, distance between lesions and physiological length. Results showed that none of these other factors correlated significantly with the extent of serial stenosis underestimation $(\mathrm{P}>0.05)$. Furthermore we found no relationship between the finding of pressure-recovery and the extent of serial stenosis interplay. 


\section{Developing Algorithms to Predict FFR true}

Our study showed total FFR and the magnitude of the pressure drop $(\Delta P)$ to be the strongest determinants of error in determining the physiological significance of each lesion. Based on these findings, two solutions were created to predict the true FFR of each stenosis; one empiricallyderived from regression modelling of the results from this study and the second from mathematical modelling of $\Delta P$ and $P d$ from theoretical principles.

\section{Empirical Regression Model}

Based on the correlations observed in our study, multiple linear regression was performed to predict $F_{\text {true }}$ (i.e. residual FFR were the stenosis in question present in isolation) from $\Delta F \mathrm{FR}_{\text {app }}$ following a fixed-rate pullback manoeuvre, using total vessel FFR as an input. This equation was created in a randomly selected derivation cohort of 32 and is summarised as follows:

$$
\text { FFRpredicted }=0.9-\left(\Delta \mathrm{FFR}_{a p p}-\mathrm{TotalFFR} * 0.11\right)
$$

Whilst such a regression model is constrained by the parameters used in this experiment, we were able to perform proof of concept validation in the remaining 20 tandemly diseased tubes. We found the initial discrepancy versus $\Delta \mathrm{FFR}_{\text {true }}$ was $17.1 \%(0.036 \pm 0.048)$ and improved significantly to $2.1 \%(0.005 \pm 0.04)$ with this equation, albeit still with a large degree of variance (Figure 6.7$)$.

\section{Theoretical Model}

The relationship between pressure and flow is curvilinear, but it has been established that most physiological measurements during hyperaemia will fall within a relatively linear part of the curve. Our theoretical model of FFR estimation was therefore based on the assumptions of a linear pressure-flow relationship. In this condition, the haemodynamic equivalent of Ohm's law is applicable, whereby the individual resistances of stenoses and the distal circulation stay fixed regardless of other stenoses being removed. Under these conditions, the theoretical FFR can be derived, without the need for a wedge pressure if we assume the variability of collateral flow across intermediate stenoses is minimal (see Supplementary Appendix for full details): 


$$
F F R_{\text {predicted }}=1-\frac{\Delta P}{P_{d}+\Delta P}
$$

In this equation, $\Delta \mathrm{P}$ refers to the pressure drop across a lesion in the presence of serial disease and $\mathrm{P}_{\mathrm{d}}$ refers distal pressure. This relationship applies for both proximal or distal lesions and depends neither on the perfusion pressure $\left(P_{\mathrm{a}}\right)$, nor the distal resistance $\mathrm{R}$, and is solely a function of the measured pressure values. Using this equation, evaluation against the $\mathrm{n}=20 \mathrm{in}$ vitro validation cohort yielded a markedly improved discrepancy of only $0.6 \%(0.006 \pm 0.02)$ against $\Delta \mathrm{FFR}_{\text {true }}$ measurements (Figure 6.7).
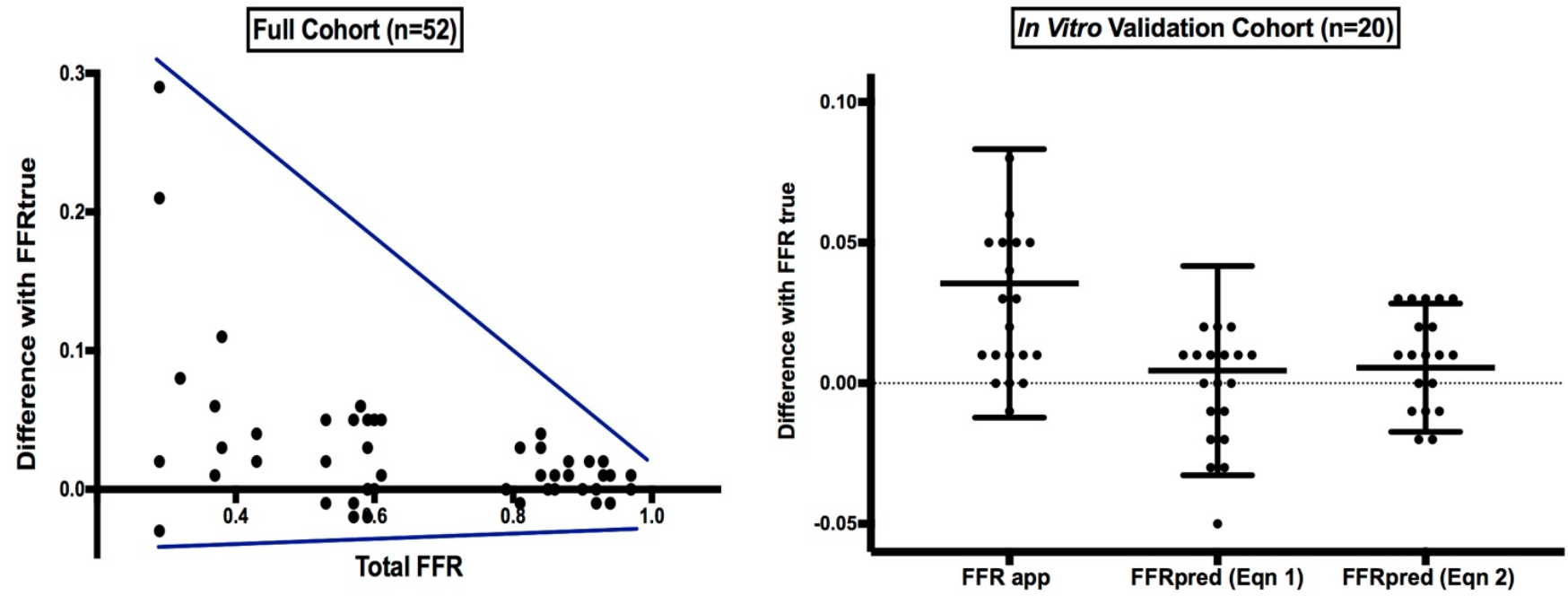

Figure 6.7: Results Demonstrating the Error in Assessing Pressure Gradients in Serial Disease and How This Can Be Corrected by Applying Correction Equations Generated From In Vitro Study. Left: In the full cohort of 52 tandemly diseased phantoms (with corresponding tube of stenoses in isolation), results demonstrated a significant $(\mathrm{P}<0.001)$ underestimation of FFR true (the more the data-point is above the $\mathrm{x}$-axis, the greater the underestimation of a stenosis). The difference between $\Delta F F R_{a p p}$ and $\Delta F F R_{\text {true }}$ and the variance of this difference was greater with increasing burden of total disease in the vessel (lower total FFR). The blue guidance lines represent the trend in increasing data heteroscedasticity with lower total FFR. Right: In a randomly-selected validation cohort of 20 phantoms, the error in estimating FFR true was significantly reduced with the statistical regression equation (Eqn 1) with the variance and difference even further reduced using the theoretical correction equation (Eqn 2), based on knowledge of $\mathrm{Pd}$ and $\Delta \mathrm{P}$ across the stenosis. 


\section{Discussion}

The main findings of this study are: 1) A physiologically representative model of serial CAD can be created in vitro using a continuous flow model based on 3-D printing. 2) Within our model, we have demonstrated that stenosis underestimation commonly occurs when physiologically assessing serial CAD, with discrepancy proportional to cumulative disease burden. 3) Using a theoretical solution derived from our in vitro experiments, we have created and validated an equation to predict to functional significance of lesions, using readily available data from routine pressure-wire pullback measurements.

The unique benefits of precisely controlling lesion geometry with 3D-printing have enabled us to overcome many limitations of previous work in the field, where stenoses were created with external ties and clamps ${ }^{27,77}$. This has enabled us to show that the factors that most influence the error when predicting individual lesion significance in serial disease are total vessel FFR, average $\%$ diameter stenosis of the tandem stenoses and $\Delta \mathrm{P}$ across the lesion is question. Other factors such as lesion separation and lesion length appear less important, with a statistically nonsignificant relationship with lesion underestimation. These findings are unsurprising as the $\%$ luminal narrowing caused by a stenosis contributes to both the linear and square coefficients within the equation that relates stenosis geometry to the pressure drop across it and as stated by Poiseuille's law, the resistance to flow is related to the $4^{\text {th }}$ power of radius of the vessel ${ }^{24}$.

This study represents a significant advance in evaluating tandem stenoses. For the first time we have been able to objectively evaluate the nature of serial stenosis interplay using novel 3Dprinting of coronary artery disease. We have subsequently generated a practical and easy-to-use correction equation, not requiring the measurement of wedge pressure between lesions, that is solely based on routine pressure wire pullback measurements and shown a significant improvement in the prediction of the true physiological significance of lesions. An important 
next step would be to test this solution within a clinical cohort of vessels with serial coronary artery disease. The only previous study with clinical validation of an in vitro solution to use with FFR was in a small cohort of 32 patients but with the requirement of measuring wedge pressure between stenoses ${ }^{27,30}$. Importantly, our solution would not need wedge pressure measurements and so requires no ballooning or the need to mandate starting PCI before the optimum revascularisation strategy is chosen.

In summary, in this in vitro study, we have shown that stenosis underestimation is significant in serial CAD, regardless of whether a distal stenosis is accompanied by a more proximal stenosis or vice versa. The factors that most influence the extent of stenosis underestimation are stenosis severity (luminal narrowing), the change in pressure across a stenosis during pressure wire pullback $(\Delta \mathrm{P})$ and Total vessel FFR (a measure of cumulative disease within the vessel and distal coronary pressure, Pd). From these findings, we have developed a mathematical correction model that significantly improves the prediction of true lesion significance in serial CAD. The next steps will be to carry out clinical validation against contemporary conventional techniques within the Catheter Laboratory such as FFR pullback and as iFR pullback ${ }^{35}$ methods (chapter 7). Subsequently the clinical applicability of this solution would need testing within a clinical study (chapter 9).

\section{Limitations}

- Tandem stenoses within humans are rarely found in otherwise smooth vessels, as has been modelled within our 3-D printed phantoms. The reality is that between stenoses, diffuse atheroma acts as a continuous mild stenosis and may hamper its performance in a clinical cohort.

- An ideal model for predicting $\Delta F F R_{\text {true }}$ would be based on the varying curvilinear relationships between pressure and flow; but this would not be possible from pressure measurements alone. We acknowledge this may be one of the reasons there is still some residual error when using a correction equation that is based on a linear pressure-flow 
relationship in physiological conditions. By doing so, however, we have been able to establish a simple model utilising pressure-wire data, without the need for simultaneous flow or wedge pressure measurement, that represents a significant improvement to current methods as evident in validation cohort of 3-D printed phantoms.

- The use of resting physiological indices, particular iFR, has been growing for the assessment of serial disease, with proponents suggesting that without hyperaemia, there is less serial stenosis haemodynamic interplay. Before universal adoption, the FFR correction model would require an assessment of its performance against other physiological indices commonly used in serial disease, such as iFR.

\section{Conclusions}

This study demonstrates that estimation of the true stenosis significance in serial disease is prone to significant underestimation using FFR, regardless of whether is there is accompanying distal or proximal disease. 3D-printed modelling of tandem disease has enabled us to generate and test a mathematical equation to improve estimation of the true physiological impact of each stenosis, using readily available measurements from a routine pressure wire study. 


\section{Supplement to Chapter 6: Theoretical Model Derivation}

Consider the pressure and resistance distribution in a vessel with tandem lesions pre and postPCI (Figure below). For illustration it is assumed that the distal lesion $\left(\mathrm{R}_{2}\right)$ is treated in this derivation, however, the outcome is identical if the proximal lesion in removed instead.

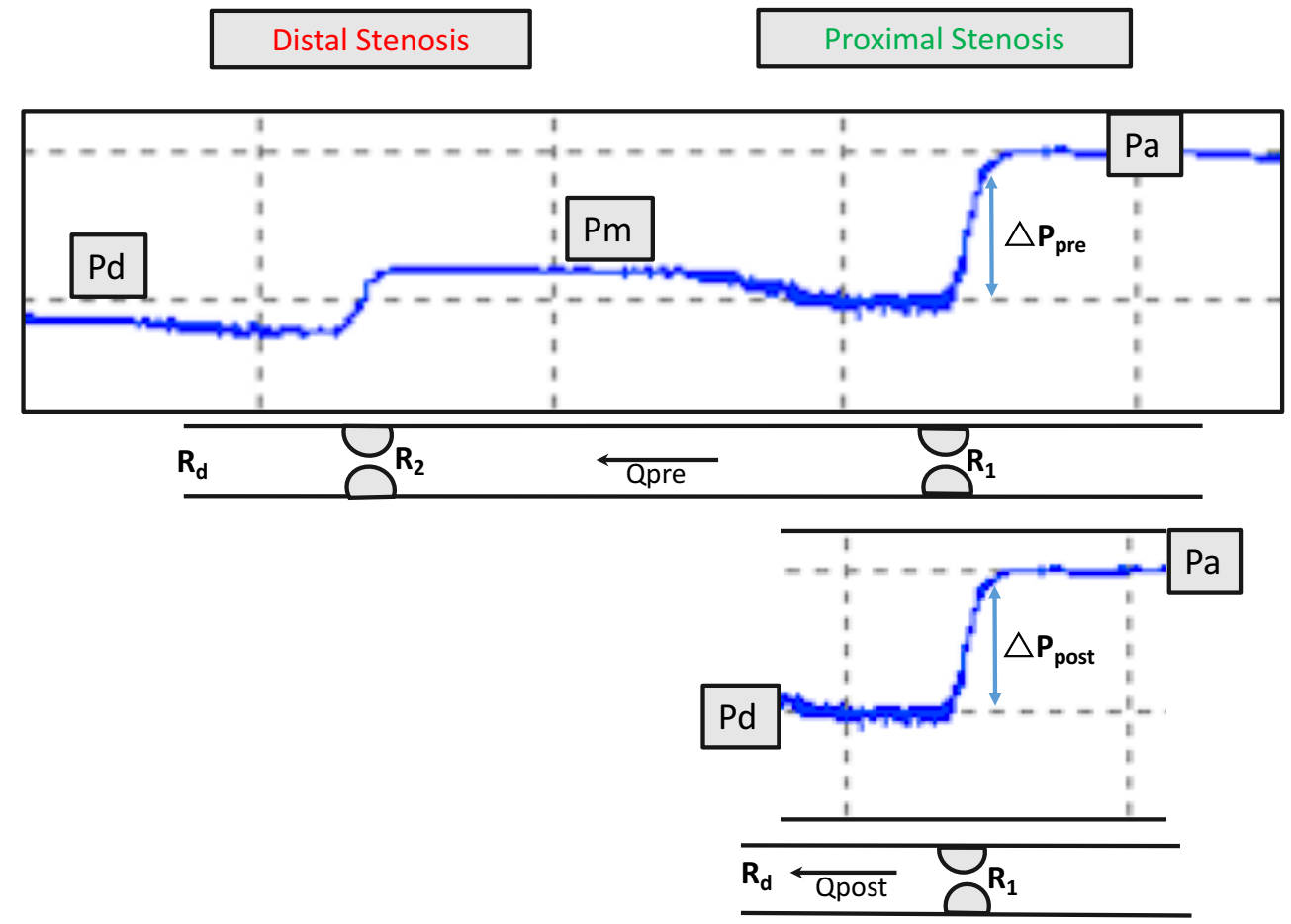

Since it is assumed that the resistance of the untreated lesion remains unchanged (i.e. $R_{1}$ and $R_{2}$ are independent) and flow remains constant throughout the vessel (i.e. no side-braches between the lesions), Ohm's law applied to pre- and post-PCI setting leads to:

$$
Q_{\text {pre }}=\frac{\Delta P_{\text {pre }}}{R_{1}} \quad \text { and } \quad Q_{\text {post }}=\frac{\Delta P_{\text {post }}}{R_{1}}
$$

which can be combined to give

$$
\frac{Q_{\text {post }}}{Q_{\text {pre }}}=\frac{\Delta P_{\text {post }}}{\Delta P_{\text {pre }}}
$$

(1)

In addition, similar expressions can be written for the distal resistance $\mathrm{R}_{\mathrm{d}}$ (which is assumed to be fixed):

$$
\begin{gathered}
Q_{\text {pre }}=\frac{P_{d}}{R_{d}} \\
Q_{\text {post }}=\frac{P_{a}-\Delta P_{\text {post }}}{R_{d}}
\end{gathered}
$$

which combine to give

$$
\frac{Q_{\text {post }}}{Q_{\text {pre }}}=\frac{P_{a}-\Delta P_{\text {post }}}{P_{d}}
$$


Equations (1) and (2) can be used together to eliminate flows

$$
\frac{\Delta P_{\text {post }}}{\Delta P_{\text {pre }}}=\frac{P_{a}-\Delta P_{\text {post }}}{P_{d}}
$$

Rearranging for $\Delta P_{\text {post }}$ yields the following expression

$$
\Delta P_{\text {post }}=\frac{\Delta P_{\text {pre }} P_{a}}{P_{d}+\Delta P_{\text {pre }}}
$$

Now, using the definition of post-PCI FFR

$$
F F R_{\text {model }}=\frac{P_{a}-\Delta P_{\text {post }}}{P_{a}}=1-\frac{\Delta P_{\text {post }}}{P_{a}}=1-\frac{\Delta P_{\text {pre }}}{P_{d}+\Delta P_{\text {pre }}}
$$

where equation (3) has been used in the last step. 


\title{
7. EXPERIMENTAL CHAPTER: COMPARISON OF FRACTIONAL FLOW RESERVE, INSTANTANEOUS WAVE FREE RATIO AND A NOVEL TECHNIQUE FOR ASSESSING CORONARY ARTERIES WITH SERIAL LESIONS
}

\author{
Presented at BCIS ACI 2018 (London) \\ Submitted to the European Heart Journal (EHJ)
}

\begin{abstract}
Acknowledgements
I would like to pay particular gratitude to the following people for their help with this section:

Dr Brian Clapp (Cardiology Dept, St Thomas' Hospital) Dr Antonis Pavlidis (Cardiology Dept, St Thomas' Hospital) Prof Simon Redwood (Cardiology Dept, St Thomas' Hospital)

Dr Haseeb Rahman (BHF Clinical Research Fellow)

Dr Matthew Ryan (BHF Clinical Research Fellow)

Ms. Lorna Simpson (Scheduling, Cardiology Dept, St Thomas' Hospital) Ms. Jean Lusengo (Scheduling, Cardiology Dept, St Thomas' Hospital)

Catheter Laboratory Staff at St Thomas' Hospital Prof Divaka Perera (Primary Supervisor)
\end{abstract}




\section{Abstract}

Background: Physiological indices such as fractional flow reserve (FFR), instantaneous wavefree ratio (iFR) and resting ratio of distal coronary to aortic pressure $(\mathrm{Pd} / \mathrm{Pa})$ are increasingly used to guide revascularisation in the catheter laboratory. Physiologically characterizing individual stenoses in serial coronary artery disease is however prone to error and an unmet need in the era of ischaemia-guided revascularisation. This study aimed to compare the accuracy of commonly used pressure-based indices (during hyperaemia: FFR; at rest: iFR and $\mathrm{Pd} / \mathrm{Pa}$ ) and a combined Doppler and pressure-derived resistance index (Hyperaemic Stenosis Resistance, hSR) in serial disease. Using these results, we aimed to develop and test a correction equation that prospectively enables more accurate physiological prediction (FFRpred) of the significance of serial stenoses. Methods: 54 patients with angiographic serial coronary stenoses were enrolled. Resting and hyperaemic coronary pressure was measured distal and proximal to each stenosis, with "apparent" FFR, iFR, Pd/Pa, and FFRpred derived for each stenosis. The "true" stenosis significance by each index was assessed following PCI of the accompanying stenosis or a large disease-free daughter branch (e.g. in the case of serial disease involving the Left Main and LAD arteries but with a disease-free Circumflex artery) . In 27 patients, Doppler Flow Velocity (APV), was also measured to calculate $\mathrm{hSR}(\mathrm{hSR}=\Delta \mathrm{P} / \mathrm{APV}$, where $\Delta \mathrm{P}=$ trans-lesional pressure gradient). Results: Mean errors for FFR, iFR and $\mathrm{Pd} / \mathrm{Pa}$, as a proportion of true measurements for each index, were $33.3 \%, 19.8 \%$ and $23.4 \%$, with underestimation inversely proportional to total vessel FFR $(\mathrm{R}=0.5, \mathrm{P}<0.001)$. This corresponded to stenosis misclassification rates of $16.7 \%, 24 \%$ and $20 \%$ for FFR, iFR and $\mathrm{Pd} / \mathrm{Pa}$ on the basis of the respective $0.80,0.89$ and 0.91 thresholds. Using FFRpred reduced the error to $14.3 \%$ with only $11.1 \%$ of stenoses misclassified. Apparent hSR correlated strongly with true hSR $(\mathrm{R}=0.87$, $\mathrm{P}<0.001$ ), with only $7.4 \%$ of stenoses misclassified. Conclusion: Assessing stenosis severity based on trans-lesional pressure gradients alone is prone to significant underestimation in serial disease, for both hyperaemic and resting pressure-based indices. The Doppler-based resistance index hSR is less prone to error but utilization is limited by difficulties obtaining Doppler traces. 
Using an FFR correction equation can reduce error to a similar extent and can be used with routine hyperaemic pressure wire measurements.

\section{Introduction}

The functional significance of coronary artery disease (CAD) is frequently assessed at the time of diagnostic angiography with pressure based indices such as fractional flow reserve (FFR) and instantaneous wave-free ratio (iFR), with good evidence that management based on these physiological measurements is superior to a strategy based on angiography alone $\mathrm{e}^{3,4,12,74,83,84}$. The data supporting physiology-guided revascularisation are largely derived from studies that only included vessels with single discrete lesions. The reality, particularly within elderly and diabetic populations, is that serial and diffuse CAD is common ${ }^{5,85,86}$. Serial CAD is important and likely to affect physiological assessment of an individual stenosis: any additional resistance proximal or distal to a stenosis will affect the trans-lesional pressure gradient at any point, with the problem further compounded by complex fluid dynamics. ${ }^{30,86}$.

The techniques most commonly used to overcome this problem is that of the FFR or iFR pullback (iFR Scout ${ }^{\mathrm{TM}}$, Philips Volcano) ${ }^{35}$. Both pressure-wire pullback techniques assume that the pressure gradient across a lesion is unaffected by the presence of the accompanying disease, with the proponents of the resting index iFR suggesting that serial stenosis interplay is less during resting conditions. Whilst these techniques are widely used and have been shown to better identify functional significance than the visual appraisal on an angiogram ${ }^{33}$, the possibility still exists that important stenoses will be misclassified with incorrect revascularisation strategies being embarked upon without knowing the true eventual significance of individual stenoses $^{86}$.

Pressure and flow-based indices of the resistance of individual stenoses are theoretically independent of a coexistent disease, with serial stenoses behaving like resistors in series within a 
circuit. Hyperaemic Stenosis Resistance (hSR) has been shown to be more accurate than single modality indices at detecting reversible perfusion defects seen using non-invasive modalities ${ }^{21}$ and has been previously been proposed not only as the best stenosis-specific physiological index $^{22}$, but also as an independent reference-standard to assess the accuracy of physiological indices of stenosis significance ${ }^{87}$. Resistance indices such as hSR, despite their inherent strengths, have never been assessed in serial CAD, with widespread utilization and further studies likely to be limited by the technical challenges of routine measurements of Doppler flow velocity.

This study aimed to assess the accuracy of indices based on trans-lesional pressure gradient alone at predicting the true significance of individual stenoses, in the presence of serial disease. The diagnostic accuracy of these indices was also compared to those of the resistance index $\mathrm{hSR}$, with the aim of testing the hypothesis that serial stenoses can behave as resistors in series. Based on this, we also aim to derive, validate and test the value of a correction equation to predict true FFR (FFRpred) from routine pressure-wire pullback.

\section{Methods}

\section{Study Population}

Patients undergoing elective angiography+/-PCI for stable angina were enrolled. Patients were chosen based on having 2 stenoses ( $>30 \%$ diameter stenosis by quantitative coronary angiography, QCA) separated by a normal segment of at least $10 \mathrm{~mm}$, where the operator would consider treating the stenoses separately. Exclusion criteria were: patients with previous coronary artery bypass grafting, recent acute coronary syndrome ( $<4$ weeks $)$ and significant valvular disease. All patients were enrolled at Guys and St Thomas' NHS Foundation Trust. All patients provided written informed consent and ethical approval was obtained from the UK Health Research Authority and local Research Ethics Committee (Ref:15/LO/2011). 
Conventional coronary angiography was performed via the right radial artery or right femoral artery. All patients received 1-2mg of diazepam before local anaesthetic was administered and arterial puncture took place. Those patients who had radial access received $0.5-1 \mathrm{mg}$ of Isosorbide dinitrate into the radial artery before cardiac catheters were advanced. All patients received prior loading with two antiplatelet agents and intra-arterial heparin to maintain an activated clotting time of greater than $250 \mathrm{~s}$ seconds. Intracoronary isosorbide dinitrate $(500 \mathrm{mcg})$ was administered prior to all pressure wire recordings. All research recordings were made via guiding catheters. In the large majority of cases $6 \mathrm{~F}$ catheters were used, however in some patients in whom Left Main stem revascularisation was planned, $6 \mathrm{~F}$ catheters were upgraded.

\section{Pressure Wire Pullback Measurements}

Guidewire pressure sensors were normalized in the coronary ostia before advancement into the distal epicardial vessels. Distal wire position was documented fluoroscopically such that subsequent pullbacks and measurements were made from the same position. For the majority of cases, a fixed rate pressure wire pullback was performed from the distal to the proximal vessel during IV adenosine-induced steady state hyperaemia $(140 \mathrm{mcg} / \mathrm{kg} / \mathrm{minute}$, onset of hyperaemia adjudged using invasive pressure waveform changes ${ }^{88}$ ) using a sterile modified IVUS pullback device (Philips Volcano R100) to pull back the pressure wire at a fixed speed of $1 \mathrm{~mm} / \mathrm{s}$. Hemodynamic parameters and distal $\mathrm{Pd} / \mathrm{Pa}$ were allowed to normalise before the vessel was rewired and pullback repeated in basal conditions. The change in iFR and FFR across each stenosis within the serially diseased vessel was termed $\Delta$ iFRapp and $\Delta$ FFRapp respectively. $\Delta \mathrm{PdPa}$ was also calculated post hoc from the resting pullback.

\section{Options for Induction of Hyperaemia}

For the majority of cases, regardless of whether additional Doppler flow measurements were made that needed intracoronary (IC) adenosine, IV adenosine was used for induction of hyperaemia and hyperaemic pressure-wire pullbacks. IV adenosine-induced hyperaemia was 
adjudged to be adequately reached based on distal pressure waveform changes we have demonstrated in our study 'Is Heart Rate Response a Reliable Marker of Adenosine-Induced Coronary Hyperaemia?' (See Appendix Chapter A). In this study we demonstrated that the best determinant of hyperaemia came from having 2 out of 3 of: 1) Ventriculisation of the distal pressure waveform, 2) Disappearance of distal dicrotic pressure notch, 3) Separation of mean aortic and distal pressures.

Intracoronary adenosine was used in all cases where simultaneous pressure-Doppler flow measurements were made. This was to enable Doppler signals to be optimised at each measurement point (keeping an adenosine infusion running for this period of time would be uncomfortable for the patient). Use of intracoronary adenosine in these cases enabled us to compare FFR values from intravenous and intracoronary adenosine. In keeping with established literature ${ }^{89,90}$, we demonstrated in 19 cases that FFR values obtained are not significantly different by paired analysis and correlated very well $(\mathrm{R}=0.97)$, see figure 7.1 . Based on these findings, intracoronary measurements of FFR through a vessel were considered reliable for cases where intravenous pressure-wire pullback measurements were not made.

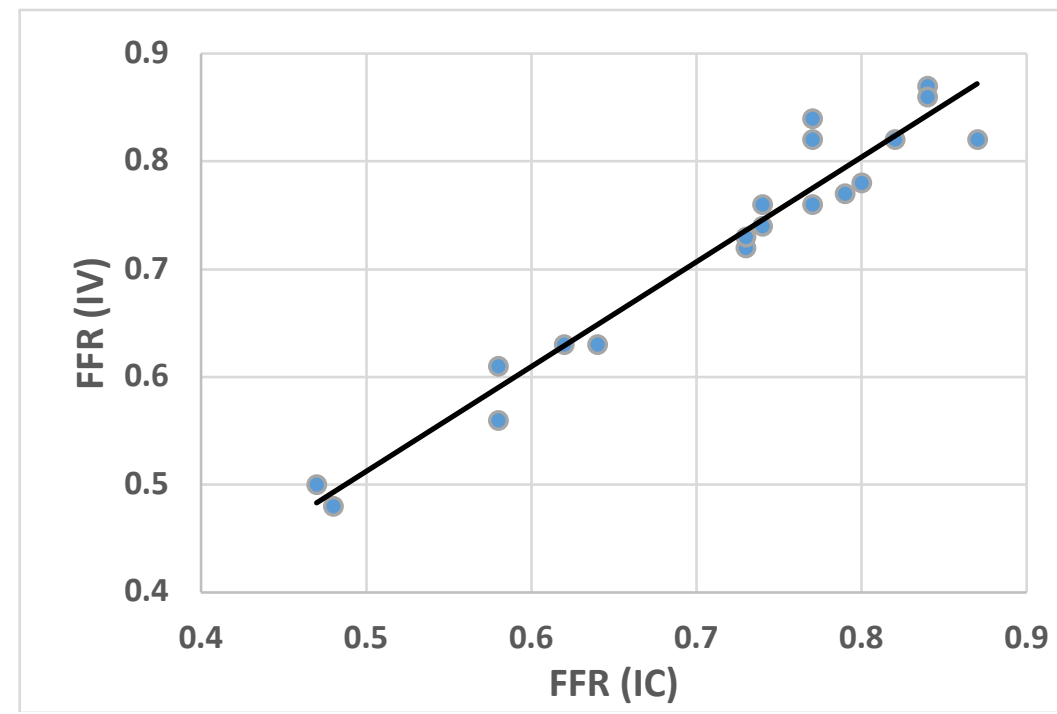

Figure 7.1 Correlation between mean FFR after intravenous (FFR $\left.{ }_{\text {iv }}\right)$ and intracoronary $\left(\right.$ FFR $\left._{\text {ic }}\right)$ administration of adenosine. $\mathrm{R}=0.97$. 


\section{Pressure/Doppler Measurements}

Following pressure wire measurements, operators judged whether pre and post-isolation measurements with a 0.014 " dual pressure-Doppler sensor guide wire (ComboWire, Volcano Philips) were safe (see figure 7.2). This wire is 0.014 " in diameter and is $185 \mathrm{~cm}$ in working length (figure 6.10) and comes with an offset and non-offset choice, where the pressure transducer and flow velocity sensor are $0 \mathrm{~cm}$ and $1.5 \mathrm{~cm}$ apart respectively. In the included studies, the softer tipped non-offset wire was used, unless the serial stenoses being studied were $\leq 1.5 \mathrm{~cm}$ apart. Before use the modular plug (for pressure) and the pin plug (for flow velocity) are connected to the pimmette of the ComboMap console (model 6800), which enables simultaneous recording and display of multiple signals.
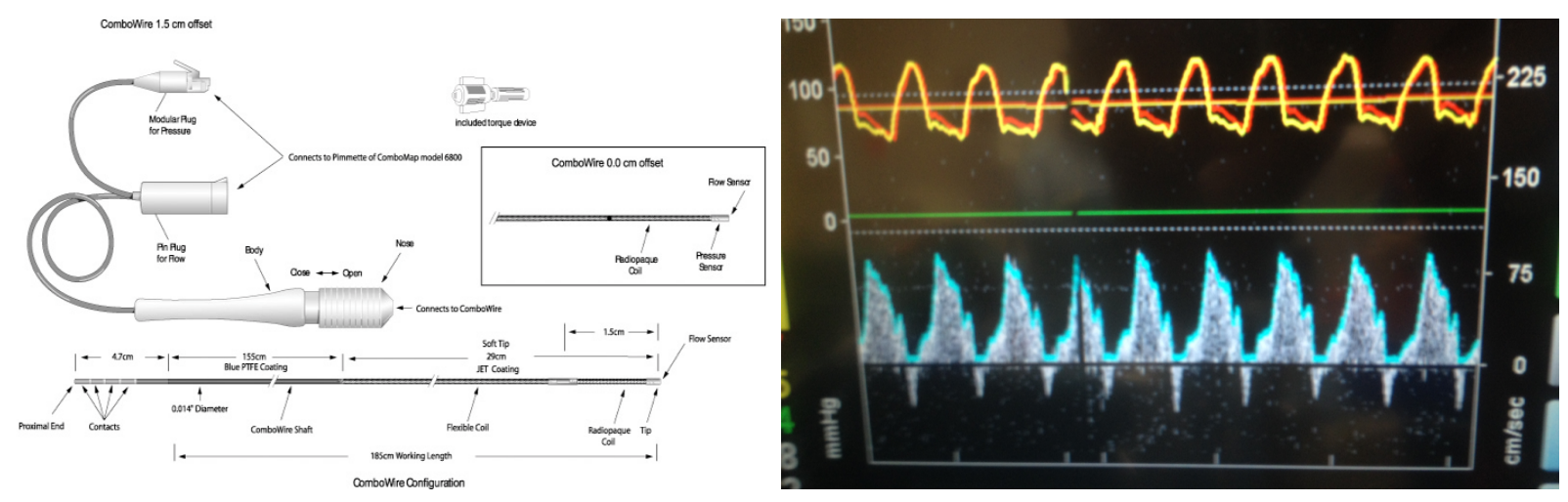

Figure 7.2: ComboWire XT technical drawing with Screen display of on ComboMap Console (Yellow trace $=$ distal coronary pressure (from the ComboWire); Red trace $=$ aortic pressure (from fluid filled catheter); Grey-scale area = Doppler flow velocity signal; Blue Envelope = Instantaneous peak velocity envelope)

Before insertion of the Combowire, the fluid-filled pressure transducer was positioned to 0mmHg and the ComboWire pressure was zeroed. The tip of the ComboWire was advanced so it just protruded out of the guide catheter and the two pressure signals were compared. If there were any differences the ComboWire pressure was normalized to the fluid-filled catheter signal. The tip of the wire was then negotiated to the distal epicardial vessel, occasionally using a standard 'workhorse' guidewire for guidance. Using fine rotational movements, the ComboWire was manipulated to obtain an optimal flow velocity signal (recognized from the shape of the 
Doppler envelope and the Doppler sound emitted from the ComboMap console). This is challenging and involves a steep learning curve for the interventional cardiologist and therefore only experienced consultant operators carried out these measurements.

Triplicate measurements of pressure and flow (average peak Doppler velocity, APV, cm/s) were taken, both at rest and with intracoronary adenosine $(100 \mathrm{mcg}$ for left coronary artery; $60 \mathrm{mcg}$ for right coronary artery), beyond the distal stenosis, at the midpoint between both stenoses and before the proximal stenosis with the Doppler signal carefully optimized at each stage. This enabled us to generate trans-lesional values for resistance (hyperaemic stenosis resistance, hSR $=(\mathrm{Pa}-\mathrm{Pd}) / \mathrm{APV}$ during hyperaemia $\left.{ }^{91}\right)$. Cases where clear Doppler traces could not be obtained were excluded from analysis.

\section{Identifying 'True’ Values of Stenosis Physiological Significance}

To compare the apparent physiological measurements with true measurements, one of two methods were used. Where possible, the pressure wire pullback and pressure-Doppler measurements were repeated following PCI of one of the 2 stenoses (the treated lesion was chosen by the operator using standard practice). In cases where serial stenoses straddled a large disease free sidebranch and where PCI was not planned (eg. left main and LAD disease where the patient was referred for CABG), the disease-free vessel was assessed to identify the true $\mathrm{Pd} / \mathrm{Pa}, \mathrm{iFR}, \mathrm{FFR}$ and $\mathrm{hSR}$. While there are some theoretical issues with this method based on different volumes of subtended myocardium, previous data has shown it to be a reliable method of assessing LMCA $\triangle \mathrm{FFR}_{\text {true }}$ provided the total FFR of the serially diseased vessel is not $\leq 0.45^{44}$. Figure 7.3 summarises the protocol. 


\section{$\underline{\text { Standard Pressure Wire Pullbacks ( } \mathrm{n}=54)}$}

Resting and Hyperemic (IV Adenosine) Pullback 'Apparent' $\triangle \mathrm{iFR}$ and $\triangle \mathrm{PdPa}$ calculated from resting pullback Apparent' $\triangle F F R$ calculated from FFR pullback
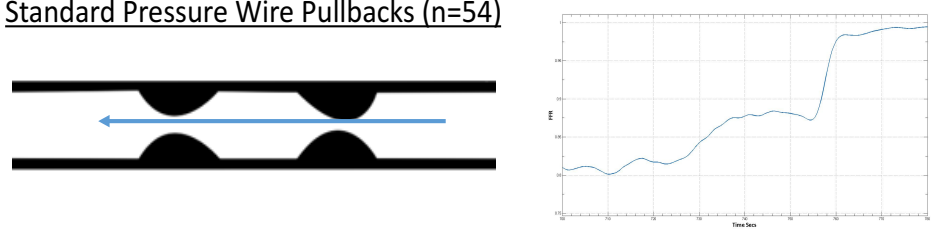

In subset ( $n=34)$ : Additional Pressure-Doppler Measurements (7 excluded)
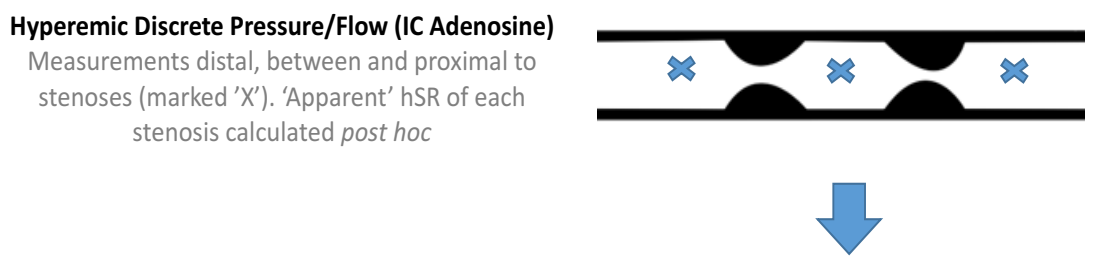

Resting and Hyperemic Pressure Wire Pullback Measurements repeated following stenosis isolaton to get 'True' iFR/PdPa/FFR values ( $n=54$ ) In $n=27$ subset: Additional Doppler Measurements following stenosis isolation to obtain 'True' $h S R$ values

Figure 7.3: Summary of catheter laboratory protocol

Testing the in vitro Derived Algorithm to Predict FFR true (FFR pred)

As described in chapter 6 , we derived the following equation to predict the true $\Delta \mathrm{FFR}$ of a stenosis:

$$
\text { FFRpred }=1-\frac{\Delta P}{P_{d}+\Delta P}
$$

In this equation, $\Delta \mathrm{P}$ refers to the pressure drop across a lesion in the presence of serial disease and $\mathrm{P}_{\mathrm{d}}$ refers to distal coronary pressure. This relationship applies for both proximal or distal lesions and depends neither on the perfusion pressure $\left(P_{\mathrm{a}}\right)$, nor the distal resistance $\mathrm{R}$, and is solely a function of the measured pressure values.

The main assumptions underpinning this equation (i.e. the applicability of the hemodynamic equivalent of Ohm's Law and that the variability of collateral flow across intermediate stenoses 
is minimal) were tested in this catheter laboratory study using measurements of total vessel and trans-lesional hSR values, as described earlier in this methods section. This clinical study also enabled validation of the equation in a clinical cohort of serial CAD and comparison with conventional resting and hyperaemic pressure-wire pullback methods of assessing serial stenoses. In the appendix relevant to chapter 7, I run through a clinical example of the equation to demonstrate how it can be of significant clinical utility.

\section{Statistical Analysis}

Data analysis was performed using SPSS Version 23 (IBM Corp., Armonk, New York). Normality was visually assessed (using histograms and Q-Q plots). Continuous data are expressed as mean +/- standard deviation and compared using paired t-tests. A two-tailed test of significance was performed for all analyses with $\mathrm{P}<0.05$ considered statistically significant. Correlations were assessed using Pearson's R with correlation coefficients displayed as R values.

\section{Results}

85 patients with serial disease were recruited into the study with 54 having successful pressurewire measurements before and after stenosis isolation. In 27 patients, combined pressure and Doppler flow measurements were possible for determination of hSR before and after lesion isolation. 7 combined pressure-Doppler cases were excluded from analysis as clear Doppler traces could not be obtained. Total vessel FFR of the 54 vessels studied was $0.72+/-0.10$. Baseline demographics and procedural details of all vessels studied is summarized in Table 7.1. There were no statistically significant differences in baseline characteristics between the whole cohort of 54 patients and the 27 patients that also had Doppler measurements. 


\begin{tabular}{|l|c|}
\hline Age, Years & $63+/-11$ \\
\hline Male & $47(87 \%)$ \\
\hline Hypertension & $36(67 \%)$ \\
\hline Diabetes & $18(33 \%)$ \\
\hline Smoker & $13(24 \%)$ \\
\hline Hyperlipidaemia & $45(83 \%)$ \\
\hline Previous PCI & $24(44 \%)$ \\
\hline Mean Lesion Severity (by QCA) & $57.9+/-11.1 \%$ \\
\hline Mean Lesion Length, mm & $10.0+/-5.8$ \\
\hline Method of Lesion Isolation & PCI $36 / 54(67 \%)$ \\
\hline Mean Lesion Separation, mm & $15.9+/-7.4$ \\
\hline & Disease Free Side-branch $18 / 54(33 \%)$ \\
\hline
\end{tabular}

Table 7.1: Summary of patient demographics

\section{Pressure-Wired Based Indices in Serial Disease}

In the 54 patients in whom the change in FFR (i.e. $\Delta \mathrm{P}$ ) was compared before and after isolation with one of the two methods, significant underestimation was observed, regardless of whether the proximal or distal lesion was considered, with a mean difference between FFRapp and FFRtrue of $0.045+/-0.05$. This corresponded to a mean error, as a proportion of the true hyperaemic $\Delta \mathrm{P}$ across of the lesion of $33.3 \%$. The extent of lesion underestimation was found to be inversely proportional to total vessel FFR: the lower the total vessel FFR, the greater the error $(\mathrm{R}=0.50, \mathrm{P}<0.001)$. 
In 50 patients, resting pressure wire pullback were also performed for calculation of the change in resting $\mathrm{Pd} / \mathrm{Pa}$ and $\mathrm{iFR}$ across each lesion before and after lesion isolation. Again, there was significant underestimation with a mean difference between apparent and true values of $0.016+/-$ 0.04 and $0.011+/-0.03$ for $\mathrm{iFR}$ and resting $\mathrm{Pd} / \mathrm{Pa}$ respectively. This corresponded to a mean error, as a proportion of the true change in index across a lesion, of $19.8 \%$ and $23.4 \%$ for iFR and resting $\mathrm{Pd} / \mathrm{Pa}$ respectively. Using FFRpred, the absolute value of underestimation fell to $0.02+/-0.04$, which corresponded to a mean proportional error of $14.3 \%$ (See figure 7.4 ).

\section{Resistance Indices in Serial Disease}

In a subset of 27 patients, apparent and true trans-lesional hSR was calculated before and after lesion isolation. In order to assess whether serial stenosis stenoses behave like resistors in series, we tested whether the sum of translesional hSR values equals the total hSR in the vessel (Figure 7.5) and found the sum of the hSR values was not significantly different from total vessel hSR $(\mathrm{P}=0.07)$.

Trans-lesional hSR values when a stenosis was present alongside another stenosis (hSRapparent) was found to correlate strongly with the hSR of the stenosis in isolation (hSRtrue), $\mathrm{P}<0.001$, $\mathrm{R}=0.87$, Figure 7.6. Mean absolute error for $\mathrm{hSR}$ was $0.19+/-0.3$, which corresponded a mean relative error of $26.4 \%$, as a proportion of true hSR. 


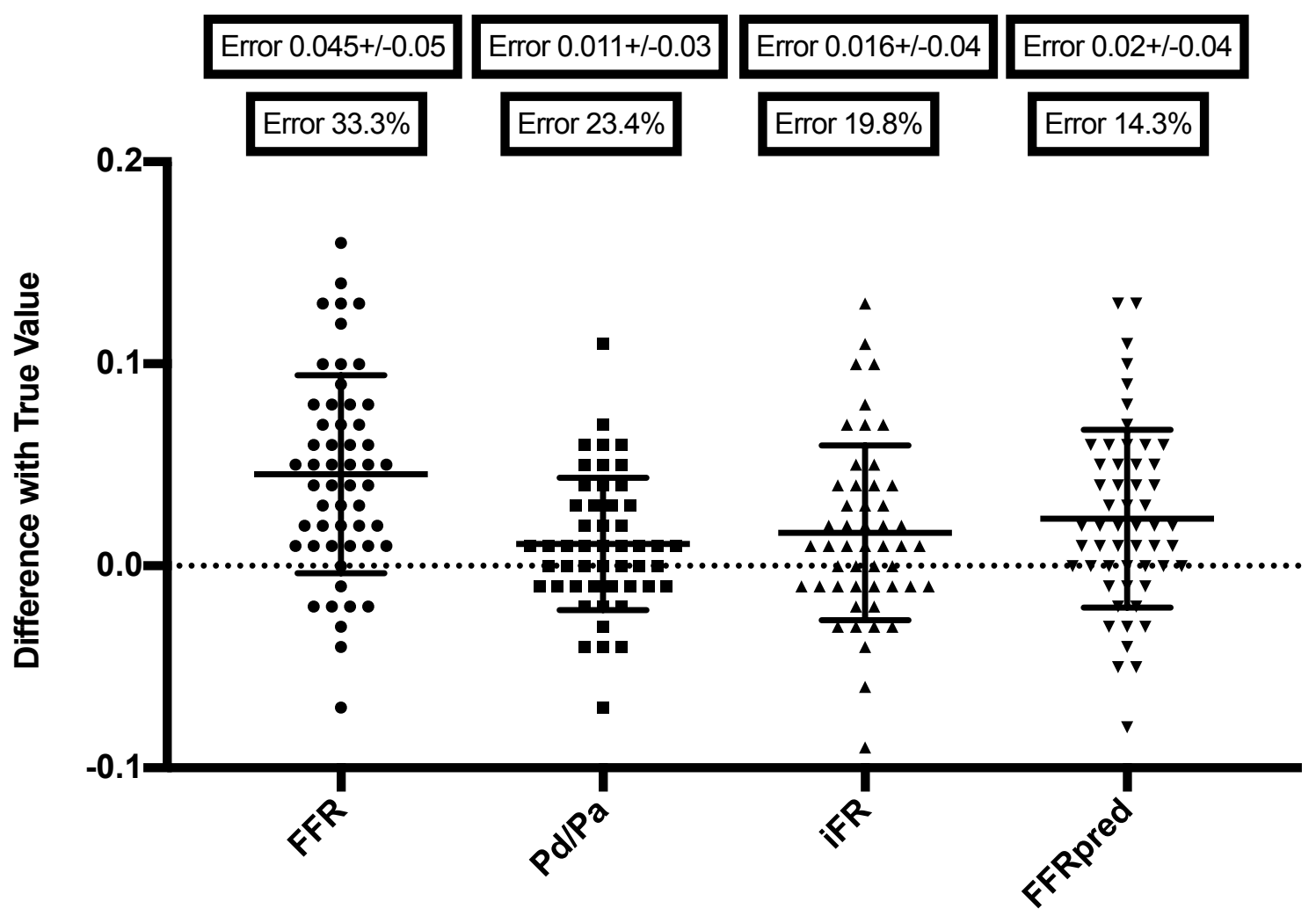

Figure 7.4: Collimated scatter plot demonstrating the error in estimating true stenosis severity (data points above zero line = lesion underestimation) when using FFR, resting PdPa, iFR and FFRpred respectively. Errors shown in absolute terms for each index $+/$ - standard deviation and also as a percentage of the true change in that index.

Using cut-off values for FFR of 0.8 , iFR of $0.89, \mathrm{PdPa}$ of 0.91 and hSR of 0.8 to identify functionally significant stenoses, we found $16.7 \%, 24 \%, 20 \%$ and $7.4 \%$ misclassification rates for lesion attributable FFR, iFR, PdPa and hSR respectively (Table 7.2).

\begin{tabular}{|c|c|c|c|c|c|}
\hline & $\begin{array}{c}\text { iFR (Using } \\
\text { iFR } \leq \mathbf{0 . 8 9})\end{array}$ & $\begin{array}{c}\text { PdPa (Using } \\
\mathbf{P d P a} \leq \mathbf{0 . 9 1 )}\end{array}$ & $\begin{array}{c}\text { FFR (Using } \\
\text { FFR } \leq \mathbf{0 . 8 0})\end{array}$ & $\begin{array}{c}\text { FFRpred } \\
\text { (Using } \\
\text { FFR } \leq \mathbf{0 . 8} \text { ) }\end{array}$ & $\begin{array}{c}\text { hSR } \\
\text { (Using } \\
\text { hSR>0.8) }\end{array}$ \\
\hline $\begin{array}{c}\text { Number of lesions } \\
\text { Misclassified }\end{array}$ & $12 / 50$ & $10 / 50$ & $9 / 54$ & $6 / 54$ & $2 / 27$ \\
\hline$\%$ Misclassification & $24 \%$ & $20 \%$ & $17 \%$ & $11 \%$ & $7 \%$ \\
\hline
\end{tabular}

Table 7.2: Table showing rates of stenosis misclassification in serial disease when using FFR, iFR, PdPa and hSR. 


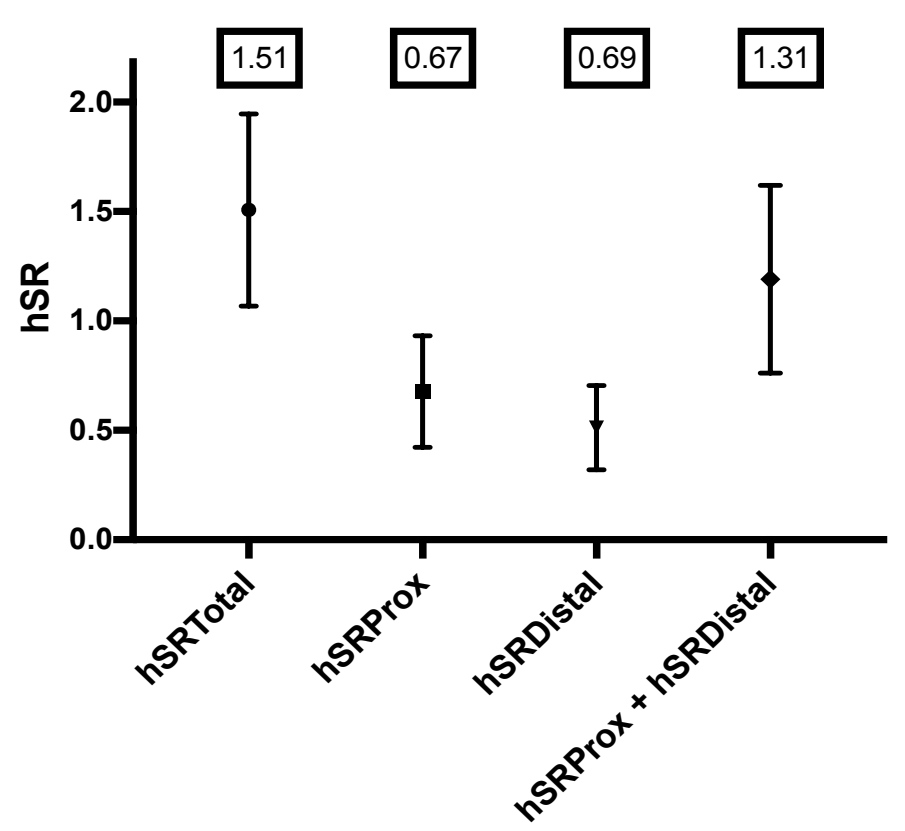

Figure 7.5: Chart plotting hSR of proximal stenosis, distal stenosis, sum of both and total vessel hSR. Values given as mean $+/$ - standard deviation. The mean sum of the hSR for both stenoses was not significantly different from total $\mathrm{hSR}(\mathrm{P}=0.07)$

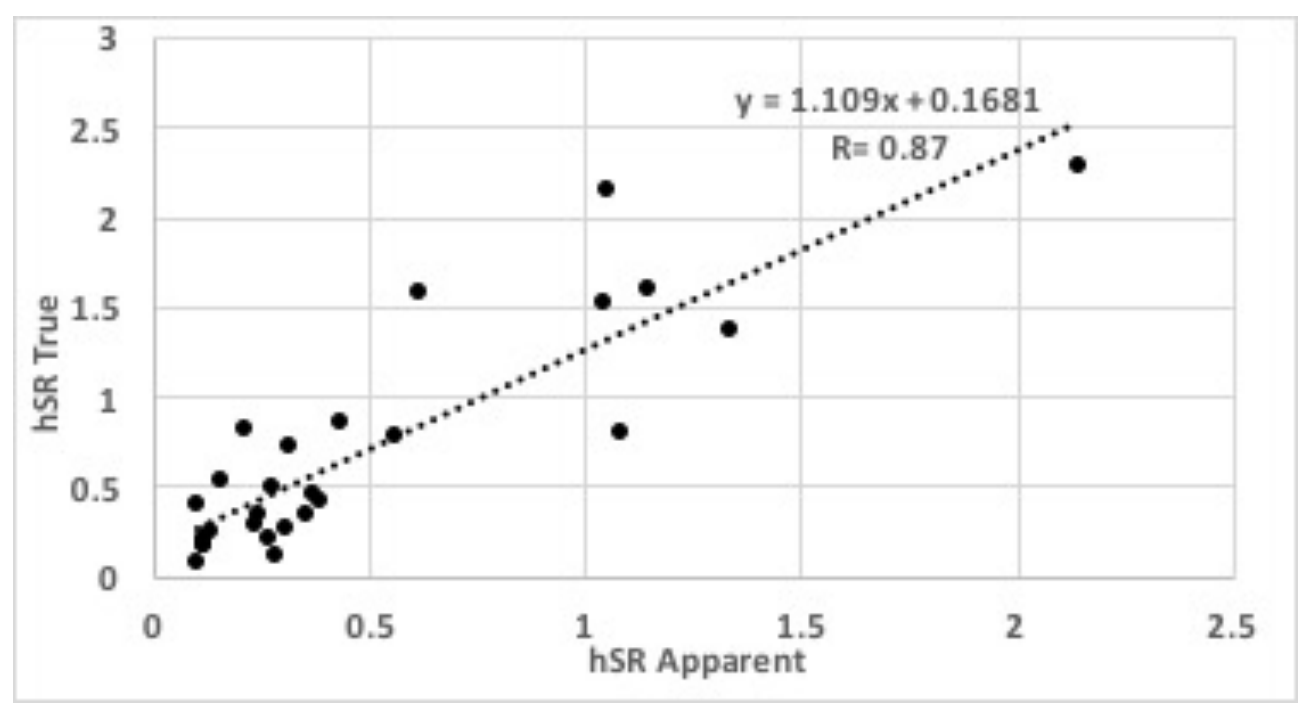

Figure 7.6: Scatterplot showing relationship between hSR of stenosis present in isolation (hSRtrue) and hSR of a stenosis when present serially alongside another (hSRapparent).

\section{Discussion}

The main findings of this study are:

i) FFR underestimates stenosis significance in serial disease, with the discrepancy amplified in vessels with a lower total vessel FFR. 
ii) Resting indices, such as iFR, also result in significant lesion underestimation. Whilst absolute errors are smaller, the error as a proportion of true change in iFR is still marked iii) All pressure-wire based indices are prone to significant error in serial disease with at least 1 in 6 stenoses being misclassified, regardless of the index used

iv) Combined pressure-flow based resistance indices appear less prone to this error, with a significant reduction in misclassified stenoses.

v) An algorithm to predict true lesion-attributable FFR in serial disease (FFRpred) can be created as we have shown that stenoses in serial disease can be assumed to behave as resistors in series. Using FFRpred significantly reduces error and misclassifications resulting from all pressure-wire based indices in serial disease.

Despite the prevalence of serial CAD and the growing utilisation of physiology-guided revascularisation, our study is the first to compare the accuracy of different physiological indices in serially diseased vessels, the first to test the value of pressure-flow based resistance indices in serial CAD and the first to validate and test the value of a correction equation to use with routine hyperaemic pressure-wire pullback measurements.

\section{Existing Data of Pressure-Derived Indices in Serial CAD}

De Bruyne et al have previously demonstrated that whilst FFR is a valid ratio for determining the hemodynamic consequences of the total burden of coronary disease within a vessel, the simple ratio cannot be applied to predict the FFR of individual stenoses if present in series, with significant errors demonstrated in their seminal studies ${ }^{27,30}$. Whilst their data suggested the proximal stenosis is more prone to error in the presence of a distal stenosis than vice versa, our data suggested there is no significant difference. De Bruyne et al subsequently derived a complex formula requiring measurement of coronary occlusive wedge pressure. Whilst wellvalidated, the method is rarely used and considered impractical ${ }^{29}$.

In the presence of no definitive solution to using FFR in serial stenoses, an increasingly used 
physiological method in serial disease is the iFR pressure pullback method with proponents of resting indices arguing that stenosis interaction is enhanced during hyperaemia and likely minimized at rest. To validate this method, Nijjer et al looked at 32 vessels with serial lesions to show a mean difference between iFR apparent (described as ' $\Delta \mathrm{iFRexp}$ ' in their study) and iFRtrue (described as ' $\triangle \mathrm{iFRobs'}$ ' in their study) of $0.016+/-0.004$. Whilst Nijjer et al state this error is small, we demonstrate a similar absolute error in our study $(0.016+/-0.04)$, but that this still corresponds to a nearly $20 \%$ error with $24 \%$ of stenoses misclassified. Given the smaller range of resting $\Delta \mathrm{P}$ vales, a 0.016 unit change has potentially a much larger relative effect on resting indices. Overall, our data suggests that all pressure derived indices are prone to significant error in serial CAD. The resting indices of $\mathrm{Pd} / \mathrm{Pa}$ and $\mathrm{iFR}$ both appear to perform better in terms of relative error but given the lower spatial resolution of these resting indices, they appear to result in similarly marked degrees of stenosis misclassification.

\section{Resistance Indices in Serial CAD}

Our data suggests that the trans-lesional resistance of a stenosis can be considered to be largely independent of disease elsewhere within the vessel, with apparent and true trans-lesional hSR correlating strongly (figure 7.6). Whilst the correlation is strong and the sum of hSR values does not appear to be statistically different from the total vessel hSR, the results do not appear strong enough to support the notion that hSR is a gold standard physiological test in serial CAD. This may be a reflection of the fact that there is some side-branching and collateral loss of flow that limits the ability of serial stenoses to behave perfectly as resistors would in an electrical circuit. Nonetheless, hSR does appear to be more robust than pressure-derived indices in predicting the true physiological significance of individual lesions, with only $7.4 \%$ of stenoses misclassified in the presence of accompanying serial disease, when compared to the true hSR of a stenosis with the lesion in isolation (table 7.2). Universal utilisation of such indices are however limited by challenges of obtaining clear intracoronary Doppler traces.

The relative independence of trans-lesional hSR values that we have shown in this study lends 
support to the methodology we have used to derive a correction equation to use with FFR pullback methods. This validity of the equation to correct for the errors from hyperaemic pressure wire pullback is further strengthened by showing that by using this equation, the relative error in estimating true stenosis FFR values is significantly reduced, with a marked decrease in the number of misclassified stenoses.

\section{Clinical Implications and Future Direction}

Our work suggests identifying the individual physiological significance of stenosis is prone to error, particular when the total ischaemic burden in a vessel is high (i.e. low FFR or iFR). Operators should be wary of this when carrying out FFR and iFR pullbacks and be aware that both hyperaemic and resting indices (including iFRScout ${ }^{\mathrm{TM}}$ ) are prone to significant error with stenoses often being wrongly misclassified in the presence of serial stenoses. Pressure-flow based resistance indices are less prone to this error, but are still prone to a degree of error with universal utilization limited by difficulties in obtaining intracoronary Doppler traces.

An easy to use correction equation to use with pressure-derived indices, not requiring measurement of intracoronary wedge pressure, has been derived and validated in this study. This solution is based solely on routine pressure wire measurements of $\Delta \mathrm{P}$ and total vessel FFR and significantly reduces error, although not to the same extent as for the 3-D printed in vitro phantoms (chapter 6). The true clinical utility of this solution now needs assessing in a prospectively planned multi-centre clinical utility study.

\section{Limitations}

Identifying a reference standard, against which all methods of physiologically assessing serial CAD, is a challenge. In this study we used the reference standard of the change in index across a stenosis when present in isolation, with lesions isolated by PCI or disease free sidebranch. Whilst these methods are established, errors are still possible: the post-PCI effects on microvascular tone are not yet established with conflicting views ${ }^{92,93}$ and the disease-free side- 
branch method is prone to small errors particular with severe disease in the main serially diseased vessel ${ }^{44}$.

This is a relatively small study, albeit the largest to examine the various existing and novel strategies in the physiological assessment of serial CAD. A larger study in a broader range of serial and diffuse coronary artery disease, together with an assessment of differing clinical practices when using each index, would be an important next step in truly understanding the value of different indices and novel solutions.

The results of the correction equation are not as impressive as in the 3-D phantom cohort (chapter 6). This may be a result of the fact that in real-life clinical cases of serial disease, stenoses have more complexed geometry with diseased side-branches between stenoses. Nonetheless, the clinical validation of this pragmatic novel solution does show a significant improvement to the performance of contemporary indices (resting and hyperemic).

\section{Conclusions}

Pressure-derived indices, both resting and hyperaemic, significantly underestimate stenosis significance in serial disease, with discrepancy proportional to cumulative disease burden. Pressure-flow based resistance indices are less prone to this error, but universal utilization is limited by difficulties in obtaining intracoronary Doppler traces. Based on these findings we describe a correction equation, to use with routine hyperaemic pressure-wire measurements, that reduces the error of pressure-derived indices to a similar extent and should be considered in routine clinical cases of serial coronary artery disease. 


\section{Supplement to Chapter 7: Clinical Case Demonstrating Correction Model}

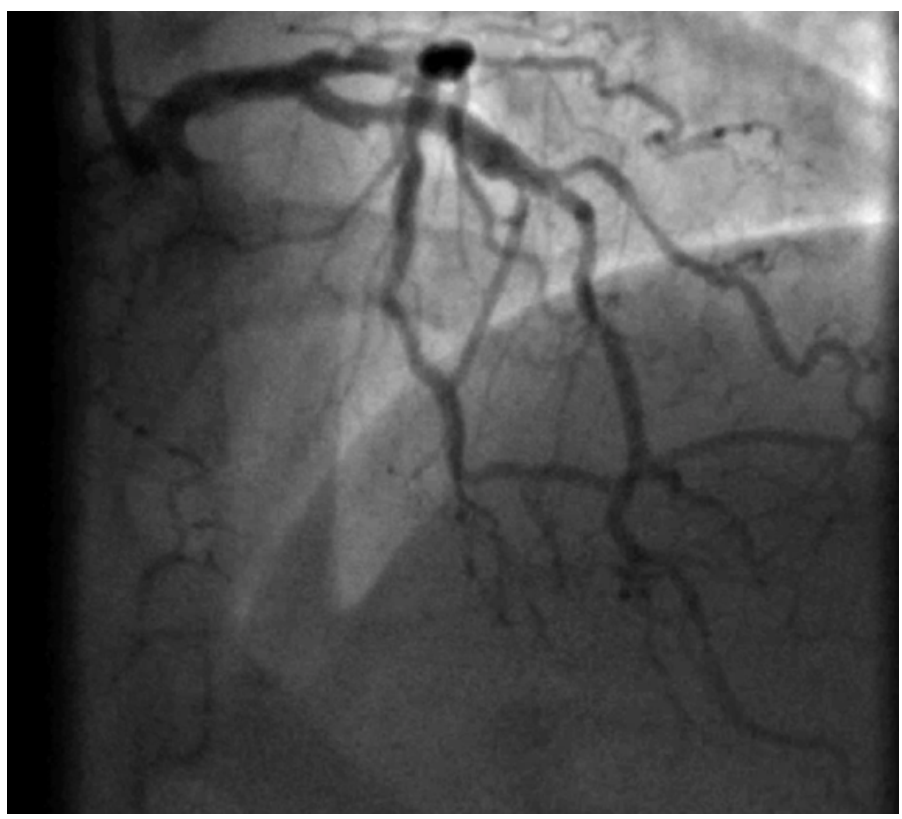

LAD demonstrating serial lesions.

Question about what the residual FFR would be due to distal lesion once proximal LAD lesion treated

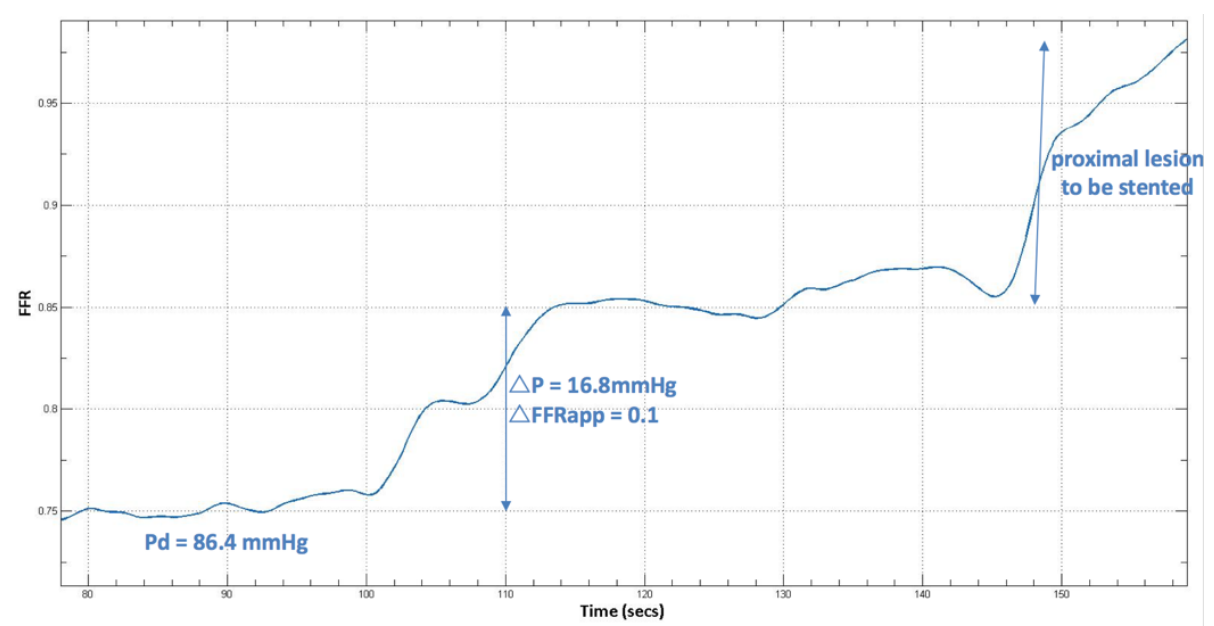

distal FFR of 0.75

(distal Pd of $86.4 \mathrm{mmHg}$ ) hyperaemic pressure-wire pullback demonstrating apparent $\Delta \mathrm{P}$ and apparent $\triangle \mathrm{FFR}$

without model, it would be estimated that residual FFR from distal stenosis only would be 0.90

$$
F F R_{\text {model }}=1-\frac{\Delta P_{\text {pre }}}{P_{d}+\Delta P_{\text {pre }}}=1-\frac{16.8}{103.2}=0.84
$$

with equation, residual FFR following PCI of proximal lesion estimated to be 0.84 


\title{
8. EXPERIMENTAL CHAPTER: NON-INVASIVE COMPUTED TOMOGRAPHY (CT) IN SERIAL CORONARY ARTERY DISEASE
}

\author{
Chapter 8.1 Published in Circulation: Cardiovascular Interventions
}

\section{Acknowledgements}

I would like to pay particular gratitude to the following people for their help with this section:

Dr Ronak Rajani (Cardiology Dept, St Thomas' Hospital)

Dr Rebecca Preston (Radiology Dept, St Thomas' Hospital)

Mr. Paul Woodburn (CT Dept, St Thomas' Hospital)

Dr Campbell Rogers (Heartflow Inc, Redwood City, CA, USA)

Dr Charley Taylor (Heartflow Inc, Redwood City, CA, USA)

Ms. Hyun Jin Kim (Heartflow Inc, Redwood City, CA, USA)

Mr. Sethuran Sankaran (Heartflow Inc, Redwood City, CA, USA)

Prof Divaka Perera (Primary Supervisor) 
As discussed in Chapter 5.5, CT enables us to make a thorough assessment of individual stenosis geometry, and therefore an accurate estimation of the frictional and separation coefficient of a stenosis (Figure 5.2). In addition, CT enables the computation and modelling of blood flow through diseased coronary arteries (Computational Fluid Dynamics, CFD). These inherent advantages of CT and the underpinning CFD theory make FFR $_{\mathrm{CT}}$-derived solution potentially ideal for unpicking serial stenosis physiology before a guidewire has entered the body.

In this chapter we therefore aim to address 3 issues:

i) Can conventional FFR $\mathrm{FT}_{\mathrm{C}}$ outputs (usually presented as a colour-contour map of FFR change down a vessel) reliably estimate true individual stenosis significance in serially diseased vessels?

ii) Based on the core principles of $\mathrm{FFR}_{\mathrm{CT}}, \mathrm{CFD}$ and coronary haemodynamics, we aim to develop and validate a novel non-invasive $\mathrm{FFR}_{\mathrm{CT}}$-derived PCI planning tool, with the aim of comparing the accuracy to estimations made from conventional FFR pullback and conventional $\mathrm{FFR}_{\mathrm{CT}}$ outputs.

iii) We have shown in vivo in Chapter 7 that serial stenoses can be considered to behave as resistors in series, with trans-lesional hSR values being largely unchanged by disease elsewhere. We therefore aimed to use the underlying principles behind $\mathrm{FFR}_{\mathrm{CT}}$ to develop and validate a CT estimation of hSR which we present in chapter 8.2. 


\title{
8.1 Predicting the Physiological Effect of Revascularisation in Serially Diseased Coronary Arteries: Clinical Validation of a Novel CT Coronary Angiography-Based Technique
}

\begin{abstract}
Background: Fractional Flow Reserve (FFR) is commonly used to assess the functional significance of Coronary Artery Disease (CAD) but is theoretically limited in evaluating individual stenoses in serially diseased vessels. We sought to characterise the accuracy of assessing individual stenoses in serial disease using invasive FFR pullback and the non-invasive equivalent, $\mathrm{FFR}_{\mathrm{CT}}$. We subsequently describe and test the accuracy of a novel non-invasive

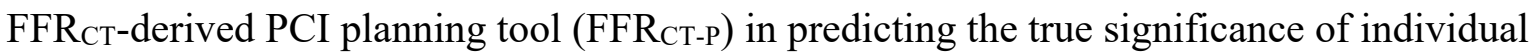
stenoses. Methods and Results: Patients with angiographic serial CAD scheduled for PCI were enrolled and underwent prospective coronary $\mathrm{CT}$ angiography with conventional $\mathrm{FFR}_{\mathrm{CT}}$ derived post hoc for each vessel and stenosis $\left(\mathrm{FFR}_{\mathrm{CT}}\right)$. Before $\mathrm{PCI}$, invasive hyperemic pressure-wire pullback was performed to derive the apparent FFR contribution of each stenosis (FFR pullback). The true FFR attributable to individual lesions ( FFR $\left._{\text {true }}\right)$ was then measured following PCI of one of the lesions. The predictive accuracy of FFR pullback, FFR CT $_{\text {and the novel technique }}$
\end{abstract}

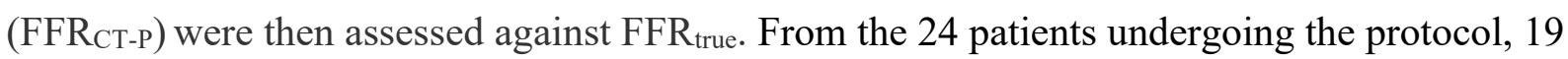
vessels had post hoc FFR $_{\mathrm{CT}}$ and FFR $\mathrm{F}_{\mathrm{CT}-\mathrm{P}}$ calculation. When assessing the distal effect of all lesions, FFR $\mathrm{CT}_{\mathrm{T}}$ correlated moderately well with invasive FFR $(\mathrm{R}=0.71, \mathrm{p}<0.001)$. For lesionspecific assessment, there was significant underestimation of FFR true $_{\text {using }}$ FFR $_{\text {pullback }}$ (mean discrepancy $0.06 \pm 0.05, \mathrm{p}<0.001$, representing a $42 \%$ error) and conventional trans-lesional FFR $_{\mathrm{CT}}(0.05 \pm 0.06, \mathrm{p}<0.001,37 \%$ error $)$. Using FFR $_{\mathrm{CT}-\mathrm{P}}$, stenosis underestimation was significantly reduced to a $7 \%$ error $(0.01 \pm 0.05, \mathrm{p}<0.001)$. Conclusions: FFR pullback and conventional $\mathrm{FFR}_{\mathrm{CT}}$ significantly underestimate true stenosis contribution in serial CAD. A novel non-invasive $\mathrm{FFR}_{\mathrm{CT}}$-based PCI planner tool more accurately predicts the true FFR contribution of each stenosis in serial CAD. 


\section{Introduction}

Coronary computed tomographic angiography (CTA) is now established as a non-invasive test for the detection of significant coronary artery disease $(\mathrm{CAD})^{57,58}$. Owing to a relatively low positive predictive value and inability to determine functional significance ${ }^{58}$, CTA has generally been used in patients at a low to intermediate risk with a view to ruling out significant $\mathrm{CAD}^{60}$. When coronary atheroma is detected, there is growing evidence that the benefit of revascularisation is only derived from targeting myocardial ischaemia ${ }^{7}$. Ischaemia-guided revascularisation has evolved to identifying vessels with functionally significant disease at the time of angiography using indices such as Fractional Flow Reserve (FFR) ${ }^{4,8,10}$. In recent years it has become possible to estimate FFR from standard coronary CTA datasets with Fractional Flow Reserve by Computed Tomography, FFR $\mathrm{CT}_{\text {. }}$ Based on computational fluid dynamics (CFD), FFR $\mathrm{CT}_{\mathrm{C}}$, has been shown to increase the positive predictive value of coronary CTA and provide clinicians with a non-invasive test to assess coronary anatomy and physiology $65,67,69$.

The studies validating both invasive FFR and FFR $\mathrm{CT}_{\mathrm{T}}$ have largely been in vessels with single epicardial stenoses ${ }^{67,94}$. In reality, CAD is often diffuse in nature with serial stenoses across the length of a vessel. Assessment of the contribution of each stenosis is challenging because physiological interplay affects the FFR attributable to each stenosis ${ }^{30,95}$.Coronary CTA enables detailed visualization of lesion geometry and a potential to evaluate the true pressure drop across a stenosis by modelling the hemodynamic interplay between serial stenoses ${ }^{95}$. FFR $\mathrm{R}_{\mathrm{CT}}$ therefore offers the potential to predict the functional significance of individual stenoses within a serially/diffusely diseased vessel, and therefore to predict the residual functional disease burden following PCI.

In this study, we describe a novel non-invasive FFR $_{\mathrm{CT}}$-derived PCI planning tool (FFR $\mathrm{FT}_{\mathrm{C}-\mathrm{P}}$ ), which models physiological interplay between stenoses. This tool, developed by HeartFlow Inc., is based on a reduced order model informed by three-dimensional simulations of blood flow to estimate 
post-PCI pressure loss. In this study we aimed to: 1) characterise the accuracy of estimating individual stenosis significance in serially diseased vessels using invasive FFR pullback and conventional FFR $\mathrm{CT}_{\mathrm{T}}$ outputs (usually presented as a colour contour map of FFR change down a vessel), and 2) validate the novel non-invasive FFR $_{\mathrm{CT}}$-derived PCI planning tool in a clinical cohort of patients with serial disease, with the aim of comparing the accuracy to estimations made from conventional FFR pullback and FFR $\mathrm{CT}_{\text {. }}$

\section{Methods}

\section{Study Population}

All patients consented had presented with symptoms of stable angina and had $\geq 2$ stenoses on an invasive coronary angiogram ( $>30 \%$ diameter stenosis by QCA). Patients were only eligible if the stenoses were separated by a normal segment of at least $10 \mathrm{~mm}$, if it were felt feasible to treat each lesion independently by PCI. Exclusion criteria were age $<18$ years, pregnancy, eGFR $<30$, previous coronary artery bypass grafting and contraindications to FFR assessment or CTA. Cardiac catheterisation was performed within 4 weeks of the prior CTA scan. The study protocol was approved by the UK Health Research Authority and local research ethics committee $(15 / \mathrm{LO} / 2011)$.

\section{CT Protocol}

All patients underwent coronary CT angiography using a 2 x 192-slice dual source CT scanner (Somatom Force, Siemens Medical Solutions, Forchheim), Germany. Eight hundred micrograms of sublingual glyceryl trinitrate was administered to all patients along with intravenous metoprolol to achieve a heart rate of $<65$ beats/min in sinus rhythm and $<100$ beats/min in atrial fibrillation. A total of $85 \mathrm{ml}$ intravenous contrast (Omnipaque, GE Healthcare, Princeton, NJ, USA) was injected at $5.5 \mathrm{ml} / \mathrm{s}$ ) by a power injector into the antecubital vein. Descending aorta contrast triggered, prospective electrocardiogram (ECG) gated scanning with adaptive padding was then performed in a single breath-hold technique after a 10-12 second delay. The scanning 
parameters included a heart rate dependent pitch of $0.2-0.45$ and a gantry rotation time of $250 \mathrm{~ms}$. The tube voltage was selected semi-automatically and automated exposure control was used for the tube current. Image slices were reconstructed using a medium sharp kernel (Bv40), using model-based iterative reconstruction strength level 3 (ADMIRE; Siemens Medical Solutions, Forchheim, Germany).

\section{FFR $_{C T}$ Protocol}

FFR $_{\mathrm{CT}}$ analysis was performed by HeartFlow, who were blinded to the invasive angiographic and physiological data. FFR $\mathrm{CT}_{\mathrm{T}}$ data were derived post hoc from the CTA dataset using methodology that has been previously described ${ }^{62,67}$. In summary, FFR ${ }_{\mathrm{CT}}$ technology involves extraction of a patient-specific geometrical model of the coronary arteries from CTA data.

Subsequently, this is combined with population-derived physiological models and computational fluid dynamics techniques to solve the governing equations of blood flow velocity and pressure under simulated hyperaemic conditions ${ }^{62}$. FFR Ст physiological models are based on three core scientific principles: an allometric scaling law relating coronary flow to myocardial mass and vessel lumen volume, the principle of flow regulation of vessel size, and an assumption of the predictable reduction of microvascular resistance with maximal hyperaemia ${ }^{96}$.

In this study, HeartFlow FFR $\mathrm{CT}_{\mathrm{T}} \mathrm{v} 2.7$ was used. This software runs on the Amazon Web Services (AWS) cloud and combines deep-learning artificial intelligence methods ${ }^{97}$ to aid in identifying the lumen boundary, physiological models that incorporate vessel lumen volume, myocardial mass data and hybrid 3D-1D computational fluid dynamics methods ${ }^{96}$. Normal HeartFlow

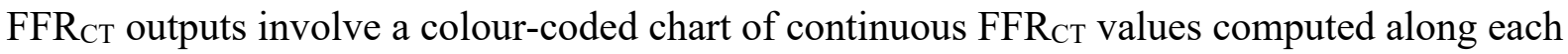
vessel. The apparent change in these discrete values across each stenosis within the serially diseased vessel were used to define the attributable $\mathrm{FFR}_{\mathrm{CT}}$ for each stenosis within the serially diseased vessel, using conventional $\mathrm{FFR}_{\mathrm{CT}}$ methods without the planning tool. 
To enable the fast recalculation of FFR $\mathrm{CT}_{\mathrm{C}}$ for different planning configurations of PCI, an accelerated method for updating FFR $\mathrm{CT}_{\mathrm{T}}$ was used (based on a reduced order model derived from computational fluid dynamics simulations). Without compromising on the accuracy of FFR $\mathrm{CT}_{\text {, }}$ this approach is able to update $\mathrm{FFR}_{\mathrm{CT}}$ solutions in real-time in response to changes in lumen geometry. Initially, idealized vessel lumen radii are calculated by first evaluating \% diameter stenosis and subsequently calculating the radius at which there would be no lumen narrowing (i.e. to achieve a \% stenosis of zero). Following this, a patient-specific idealized model is constructed using the idealized lumen radii. Finally, a CFD-derived reduced order model is used to calculate the updated FFR $\mathrm{CT}_{\mathrm{T}}$ values.

This method involves CFD-derived reduced order model based on a flow-dependent resistance model for the epicardial arteries, $R=R_{\text {in }}+R_{s l} Q$, where $R_{\text {in }}$ and $R_{s l}$ are the intercept and slope of the resistance-flow relationship, $Q$ is the flowrate and $R=\frac{\Delta P}{Q}$ is the resistance posed by the epicardial vessel to blood flow. Since these quantities also depend on the epicardial geometry, two different flowrates are used to calculate $R_{i n}$ and $R_{s l}$. In each of the original and idealized geometries, two different boundary conditions, the first being hyperaemia as described in the previous section and the second being a $40 \%$ lower resistance, are applied. If the flowrates achieved are $Q$ and $Q^{*}$, and the corresponding resistances calculated from CFD simulations are $R$ and $R^{*}$ then:

$$
R_{\text {in }}=\frac{\mathrm{R} * \mathrm{Q}-\mathrm{Q} * \mathrm{R}}{Q-Q *} \quad \text { and } \quad R_{s l}=\frac{\mathrm{R} *-\mathrm{R}}{Q *-Q}
$$

If the flow difference achieved, $Q^{*}-Q$, is too low, then the intercept and slope are replaced with a 1D CFD model parameterized by lumen area (A), viscosity $(\mu)$, density $(\rho)$, as

$$
\begin{aligned}
& R_{\text {in }}=\frac{8 \mu \mathrm{L} \pi}{\mathrm{A}^{2}} \\
& \text { and } \\
& R_{s l}=\frac{\rho \mathrm{LdA}}{\mathrm{A}^{3} \mathrm{dz}}
\end{aligned}
$$


These resistances are applied on the updated lumen geometry (where lumen radii in the stented region are replaced by their idealized values), to solve for flowrates, pressures and FFR $\mathrm{R}_{\mathrm{C}}$. For the purposes of this study, FFR $\mathrm{CT}_{\text {-P }}$ of each stenosis was derived following the retrospective application of these calculations to CTA images, blinded to invasive FFR values but with knowledge of stent size and position.

\section{Cardiac Catheterization Protocol}

Angiography was performed via the right radial artery using 6Fr catheters. Prior to catheterization, all patients received 300mg of aspirin, 600mg Clopidogrel and benzodiazepine sedation before local anaesthetic was administered. Following arterial puncture, patients received $500-1000 \mathrm{mcg}$ isosorbide dinitrate into the radial artery before cardiac catheters were advanced and a further intracoronary bolus before acquisition of standard diagnostic images.

\section{Invasive Pressure-Wire Data Protocol}

Before insertion, the pressure-wire was zeroed outside of the body, against a fluid-filled guiding catheter pressure-transducer positioned at the level of the right atrium. After insertion, the pressure sensor was normalised to aortic pressure at the coronary ostium, with the guide disengaged where necessary. The pressure wire tip was delivered to the distal part of the serially-diseased vessel, with wire position documented fluoroscopically. An IV adenosine infusion was administered at a dose $140 \mathrm{mcg} / \mathrm{kg} / \mathrm{min}$ via an antecubital vein. FFR was defined as the lowest $\mathrm{Pd} / \mathrm{Pa}$ ratio averaged over 5 cardiac cycles following the onset of adenosine ${ }^{47}$. Following documentation of FFR, the pressure-wire was pulled back to the guide catheter at a fixed speed during continuous adenosine infusion.

The 'apparent' change in hyperaemic pressure gradient across each stenosis following pressurewire pullback within the serially diseased vessel was noted with the attributable FFR termed 'FFR pullback' PCI was then performed, with the choice of stenosis treated at the discretion of the 
operator using their conventional practice. Following re-calibration, pressure-wire pullback was then repeated. The subsequent 'true' change in hyperaemic pressure gradient across the remaining stenosis in isolation was noted with the stenosis-attributable FFR termed 'FFR ${ }_{\text {true }}$ ' (i.e. FFR in the vessel following PCI of the accompanying serial stenosis). The difference between FFR true and FFR $_{\text {pullback }}$ was indicative of the degree of stenosis underestimation in serial CAD. The relationship between the extent of stenosis underestimation and 'Total Vessel FFR' (the cumulative FFR in the vessel before PCI when both stenoses were present) was also assessed for each invasive and non-invasive index.

\section{Statistical Analysis}

SPSS Version 23 (IBM Corp., Armonk, New York) and Prism Graphpad 5.0 (GraphPad Software inc. CA) were used for all analyses. Normality was assessed using a visual assessment of histograms and Q-Q plots. Continuous data are expressed as mean \pm standard deviation and compared using paired t-tests. A two-tailed test of significance was performed for all analyses with $\mathrm{p}<0.05$ being considered statistically significant. Correlations were assessed using Pearson's correlation coefficients $(\mathrm{R})$.

\section{Results}

27 patients had prospective CT coronary angiography that demonstrated serial CAD in 30 vessels. Of these, 5 patients (6 vessels) were excluded as satisfactory catheter laboratory data could not be obtained (reasons were: stenosis deemed too severe to make pressure-wire measurements, vessel tortuosity making it difficult to pass pressure-wire and an adverse reaction to adenosine). Of the remaining 24 vessels, 19 (79\%) had satisfactory computation of FFR $\mathrm{CT}_{\mathrm{C}}$ along the vessel (3 patients were excluded due to proximal stented segments and 2 owing to CTA motion artefacts that precluded post hoc $\mathrm{FFR}_{\mathrm{CT}}$ computation).

Patients included in the final analysis had a median age of 64.7 years ( $89 \%$ male). Table 1 summarizes the patient demographics and details of the serially diseased vessels. FFR of these 
vessels was $0.67 \pm 0.16$. Total vessel $\mathrm{FFR}_{\mathrm{CT}}$ of these vessels was $0.68 \pm 0.18$. The Invasive total vessel FFR showed good agreement with total vessel FFR $_{\mathrm{CT}}$, in line with previous studies ${ }^{67,69}$ (Pearson's $\mathrm{R}=0.71)$.

With FFR pullback, significant underestimation of lesion severity occurred, regardless of whether the proximal or distal lesion was considered, with a mean difference between FFR $_{\text {pullback }}$ and FFR $_{\text {true }}$ of $0.06 \pm 0.05(\mathrm{p}<0.001)$. This corresponded to a mean error, as a proportion of the true

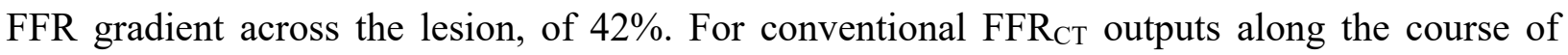
vessels, similar significant underestimation of lesion severity occurred, with a mean difference between $\mathrm{FFR}_{\mathrm{CT}}$ and $\mathrm{FFR}_{\text {true }}$ of $0.05 \pm 0.06$ ( $\mathrm{p}<0.001$ ), giving a mean error of $37 \%$.

\begin{tabular}{|l|c|}
\hline Age, Years & $64.7 \pm 12.0$ \\
\hline Male & $17(89 \%)$ \\
\hline Diabetes & $12(63 \%)$ \\
\hline Smoker & $6(32 \%)$ \\
\hline Hyperlipidaemia & $5(26 \%)$ \\
\hline Previous PCI & $15(79 \%)$ \\
\hline Lesion Severity (by QCA) & $6(32 \%)$ \\
\hline Lesion Length, mm & $58.9 \%$ \\
\hline Lesion Separation, mm & 11.3 \\
\hline
\end{tabular}

Table 8.1 Summary of patient demographics and Lesion Characteristics. Data presented as mean values \pm standard deviation with percentage prevalence where relevant. $\mathrm{PCI}=$ Percutaneous Coronary Intervention; QCA; Quantified Coronary Angiography; mm = millimetres 
Estimating True Stenosis Significance with Interactive Planner Tool

Using the newly developed interactive FFR ${ }_{\mathrm{CT}}$ PCI planning tool, we were able to estimate the FFR in the vessel (FFR $\mathrm{FT}_{\mathrm{P}-\mathrm{P}}$ ) following optimal PCI of one of the two serial stenoses (example case shown in Figure 8.1 below).

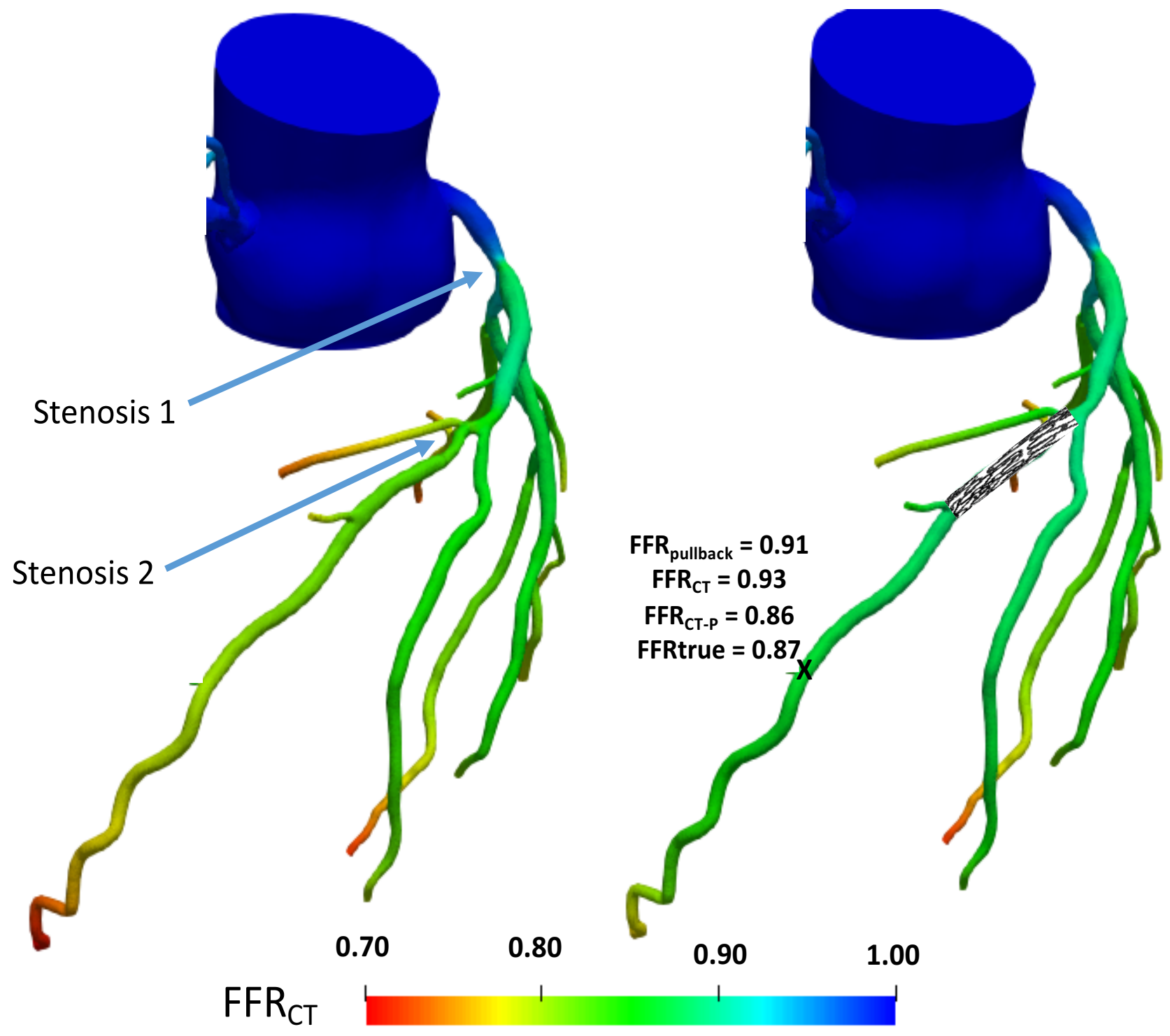

Figure 8.1: Example case showing colour contour map of FFR change in a serially diseased vessel with improvement in estimation of true stenosis significance with interactive planner Tool

Left: Example of conventional FFR $\mathrm{CT}_{\mathrm{T}}$ output for a serially diseased LAD (serial stenoses labelled) prior to simulated PCI. The FFR $\mathrm{RT}_{\mathrm{CT}}$ value between and distal to stenoses is suggested to be 0.93 and 0.80 respectively. Right: Results of true measured FFR at point ' $X$ ' after PCI of stenosis 2, comparing favourably with FFR $\mathrm{CT}_{\mathrm{P}-\mathrm{P}}$, following application of novel planner tool. Using conventional FFR $\mathrm{CT}_{\mathrm{T}}$ and $\mathrm{FFR}_{\text {pullback }}$ the underestimation would have been more significant (0.93 and 0.91 respectively) 


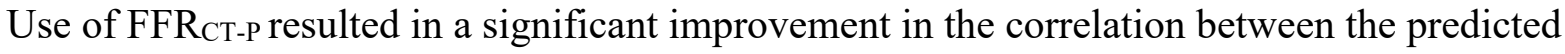
$\mathrm{FFR}_{\mathrm{CT}}$ and $\mathrm{FFR}$ true, following PCI of one of the two serial stenoses $(\mathrm{R}=0.44$ with conventional $\mathrm{FFR}_{\mathrm{CT}}$, improving significantly to $\mathrm{R}=0.75$ with $\mathrm{FFR}_{\mathrm{CT}-\mathrm{P}}$; see Figure 8.2 ). Whilst there was good

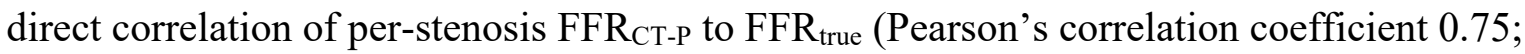
$p=0.001$; figure 8.2), in a few cases there was significant over/under-estimation, particularly when the baseline total vessel FFR was $<0.7)$.
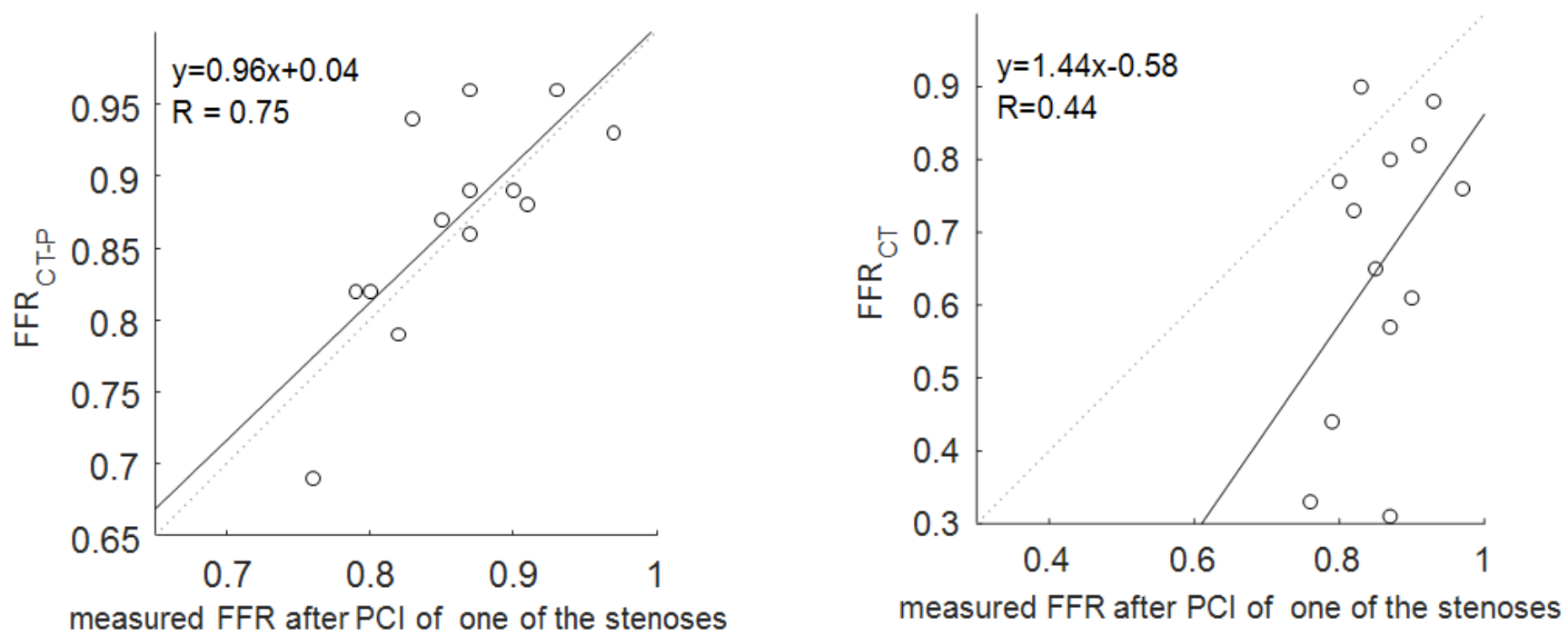

Figure 8.2: Scatterplots demonstrating how planner tool improves estimation of FFRtrue Legend: Scatterplots demonstrating improved estimation of FFRtrue across all patients with FFR $_{\text {CT-P }}(p=0.001)$. Left: Comparison of FFR ${ }_{\text {CT-P }}$ and measured FFR post PCI. Right:

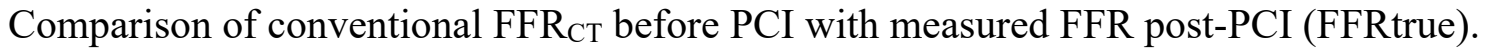

By applying the interactive planner tool on a geometry matching the stent location in the subsequent PCI, the absolute value of underestimation fell to $0.01 \pm 0.05$, which corresponded to a mean proportional error of 7\% (Figure 8.3). 


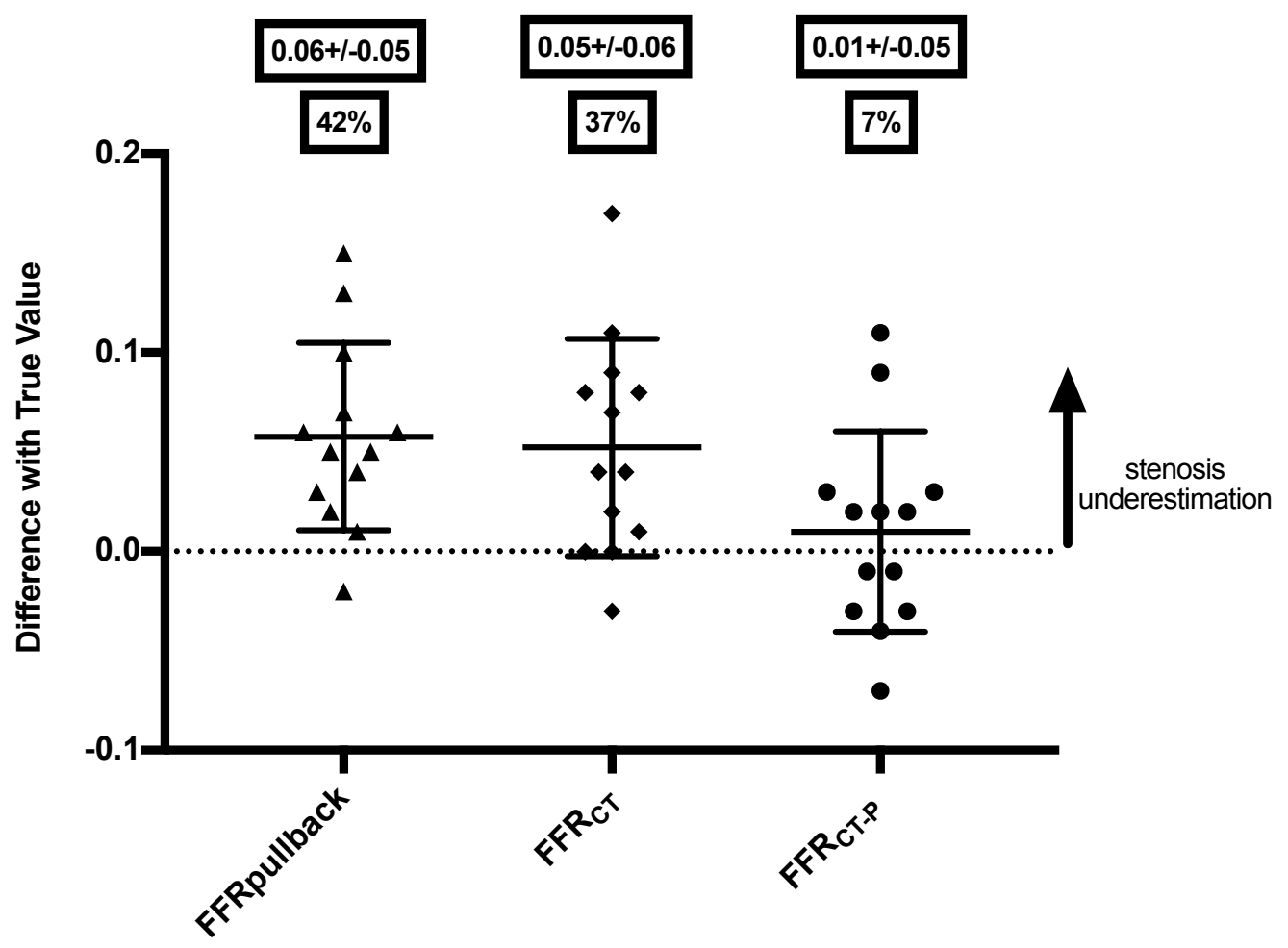

Figure 8.3: Categorical scatter plot comparing errors in estimating true stenosis significance

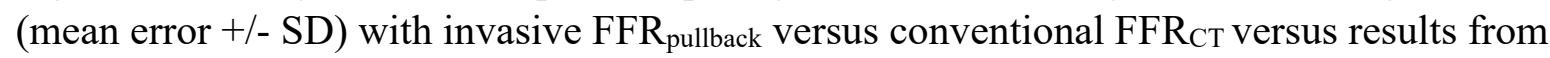

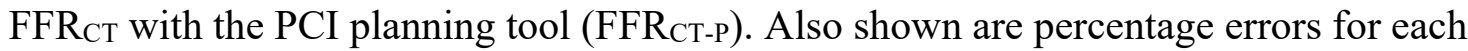
physiological tool as a proportion of the true FFR change, derived following isolation of a stenosis by PCI of an accompanying tandem stenosis.

\section{Discussion}

In this study, we have shown that (i) using an unadjusted trans-lesional gradient from conventional FFR $_{\mathrm{CT}}$ outputs leads to significant underestimation of stenosis significance in serially diseased vessels, similar to invasive FFR pullback methods and (ii) applying a novel interactive PCI planning tool (FFR CT-P), , results in a significant improvement in the accuracy of predicting the residual 'true' FFR contribution of stenoses following treatment of accompanying serial stenoses. To our knowledge, this is the first description and validation of this novel non-invasive method to predict the haemodynamic outcome of a PCI procedure.

Serial and diffuse coronary artery disease is common, particularly within ageing and diabetic populations, with estimates of $25-40 \%$ prevalence in all patients presenting for angiography ${ }^{5,6}$. 
There are however several recognised limitations of physiological assessment of serial coronary artery disease. A simple explanation for this is that a stenosis provides resistance to blood flow, manifesting as a post-stenotic drop in pressure that is magnified in hyperaemic conditions to give the true FFR when present in isolation. When another stenosis or diffusely-diseased segment, maximal hyperaemia (and therefore measurement of the 'true FFR' resulting from the stenoses) is not possible due to the additional resistance to flow, with the situation further complicated by flow turbulence when stenoses are particularly severe, close together and non-concentric ${ }^{29,95}$. The techniques previously described to overcome these limitations have been complex; requiring measurement of coronary wedge pressure ${ }^{30}$ or the presence of a disease-free daughter branch when serial disease involves the Left Main Coronary Artery ${ }^{44}$. While it has been suggested that resting indices (eg iFR pullback) might result in smaller absolute errors than FFR in this setting ${ }^{35}$, this is likely to be a reflection of the different operating range of resting indices, which are also prone to significant error.

At present, conventional $\mathrm{FFR}_{\mathrm{CT}}$ outputs are presented as colour coded maps of coronary arteries showing how FFR $\mathrm{CT}_{\mathrm{T}}$ values change down the vessel. These colour-coded gradients of FFR $\mathrm{CT}_{\mathrm{C}}$ are designed to mirror the changes in measured FFR one would expect with a hyperaemic pressurewire pullback within the catheter laboratory. In keeping with this, our results show that unadjusted trans-lesional gradients from these conventional FFR $_{\mathrm{CT}}$ outputs show a similarly significant degree of stenosis underestimation in serially diseased vessels, as is the case with invasive FFR pullback. Given that the physiological interplay largely depends on lesion geometry, which in turn

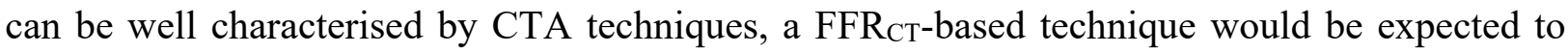
have greater potential at predicting the true physiological contribution of an individual stenosis, with the added appeal of being a non-invasive technique. Based on this, the $\mathrm{FFR}_{\mathrm{CT}}$-derived PCI planning tool has been developed to allow real-time recalculation of residual FFR after simulating PCI, with the operator able to choose simulated stent position and length. 
Despite the large and significant reduction in mean error using the novel FFR $\mathrm{CT}_{\mathrm{C}}$ method FFR $_{\mathrm{CT}}$ $\mathrm{P})$, the variance of the error due to serial disease is still appreciable ( $\mathrm{SD}+/-0.05)$, particularly when the cumulative burden of disease in the vessel is high (i.e. total FFR low). These findings are consistent with previous meta-analyses of FFR $\mathrm{CT}_{\mathrm{C}}$ diagnostic performance that have shown reduced accuracy with more severe stenoses ${ }^{98}$. This may be as a result of limited CT visualisation of stenosis geometry when stenoses are severe but also variability in microvascular function which is difficult to predict from a non-invasive functional-anatomical test. In addition, the geometry of the untreated lesion can change post-PCI due to a higher operating pressure and can lead to unaccounted changes in FFR $\mathrm{CT}_{\mathrm{T}}$. There are several additional issues when using $\mathrm{FFR}_{\mathrm{CT}}$ in planning intervention that may limit the utility of such a non-invasive tool: Firstly, there is a need to have an appropriate CT scan within a few weeks of the planned PCI. Secondly, not all prospectively acquired CT scans are of adequate quality for FFR $\mathrm{CT}_{\mathrm{CT}}$ computation. Within our study, around 20\% of cases could not have post hoc $\mathrm{FFR}_{\mathrm{CT}}$ computation due to issues such as phase misalignment. This was a rate similar to the NXT trial where $13 \%$ of CT scans were unsuitable for analysis owing to 'inadequate image quality' issues such as phase misalignment, stent-related artefacts and blooming $^{67}$.

Despite these factors, in patients that are able to have an adequate quality prospective CT coronary angiogram prior to planned PCI, FFR $\mathrm{CT}_{\mathrm{P}-\mathrm{P}}$ still represents a significant improvement to current methods of physiologically assessing serial CAD. Furthermore, it has the potential to complement contemporary methods of planning PCI, particularly in patients with serial/diffuse CAD, without requiring the invasive positioning of a coronary guidewire. Further multi-centre data will be needed to evaluate this tool and its impact of clinical decision-making.

\section{Limitations}

The study is limited by its relatively small sample size although it is the first study to provide validation of FFR $\mathrm{CT}_{\mathrm{C}}$ and the novel interactive planner tool in serial lesions. In addition, FFR $\mathrm{R}_{\mathrm{CT}}$ has not been validated for patients with previous coronary artery bypass, previous stenting within the 
same vessel or significantly abnormal left ventricular function, and therefore by extension we excluded these patients and we cannot extrapolate the results from the FFR $\mathrm{R}_{\mathrm{CT}} \mathrm{PCI}$ planning tool to those patient groups.

\section{Conclusion}

FFR $_{\mathrm{CT}}$ provides a comparable estimation of FFR values obtained from routine invasive pressurewire pullback along a vessel and is prone to a similar degree of physiological underestimation in serial coronary artery disease. In this study we provide validation for a novel non-invasive FFR CT- $^{-}$ derived PCI planning tool (FFR $\left.\mathrm{FT}_{\mathrm{C}-\mathrm{P}}\right)$ and show it to significantly reduce the error of contemporary methods in estimating true stenosis significance within serially diseased vessels following PCI of an accompanying stenosis. 


\subsection{Estimating Hyperaemic Stenosis Resistance by Cardiac CT}

\section{Introduction}

As described in earlier sections, Hyperaemic Stenosis Resistance, hSR, is an invasive index calculated by measurement of both intra-coronary pressure and Doppler flow velocity and so overcomes many of the limitations of flow-only and pressure-only based indices ${ }^{21,42,99}$. It has previously been shown to provide a more accurate assessment of ischaemia compared to other invasive physiological indices ${ }^{21}$ and has previously been used as an invasive reference standard to compare invasive pressure-derived physiological indices ${ }^{87}$. In addition, our data suggests that the trans-lesional resistance of a stenosis can be considered to be largely independent of flow conditions and resistance elsewhere within the vessel. The data suggests that hSR does appear to be more robust than the pressure-derived indices in predicting the true physiological significance of individual lesions in serial disease, with apparent and true trans-lesional hSR correlating strongly.

Despite these strengths of Doppler-derived resistance indices, both on a trans-lesional and total vessel level, universal utilization of such indices are limited by challenges of obtaining clear intracoronary Doppler traces. With advances in CT and Computational Fluid Dynamics, it is possible that hSR values can be derived using computed blood flow and pressure values. We sought to compare how total vessel and trans-lesional hSR values compare with CT-computed hSR values to see if we can validate an easier non-invasive method for assessing this valuable index.

\section{Methods}

The study population, CT protocol and Cardiac Catheterization protocols used were identical to those described in section 8.1. The cases selected for this study needed to have had invasive hSR

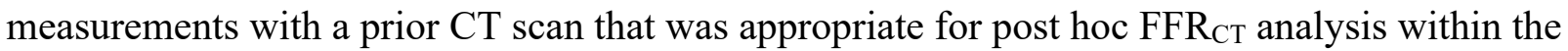


vessel of interest. From these cases, invasive hSR and CT derived hSR values were assessed, as described below.

\section{Invasive Pressure-Doppler Data Protocol}

Following diagnostic angiography, operators judged the safety of pre and post-isolation measurements with a 0.014 " dual pressure-Doppler sensor guide wire (ComboWire, Volcano Philips). In these cases, the guide wire was manoeuvred to the distal epicardial vessel following initial calibration to aortic pressure. Triplicate measurements of pressure and flow (average peak Doppler velocity, APV, $\mathrm{cm} / \mathrm{s}$ ) were taken, both at rest and with intracoronary adenosine (100mcg for left coronary artery; $60 \mathrm{mcg}$ for right coronary artery), beyond the distal stenosis, at the midpoint between both stenoses and before the proximal stenosis with the Doppler signal envelope carefully optimized at each stage. This enabled calculation of trans-lesional values for resistance (hyperaemic stenosis resistance, $\mathrm{hSR}=(\mathrm{Pa}-\mathrm{Pd}) / \mathrm{APV}$ during hyperaemia $\left.{ }^{91}\right)$. Cases where clear Doppler traces could not be obtained were excluded from analysis.

\section{CT derived $h S R$}

For each patient, in section 8.1, a three-dimensional anatomic model of the aorta and epicardial coronary arteries was generated from coronary CTA images. This model was used to create physiological models based on three core principles: an allometric scaling law relating coronary flow to myocardial mass and vessel lumen volume, the principle of flow regulation of vessel size, and an assumption of the predictable reduction of microvascular resistance with maximal hyperaemia ${ }^{96}$.

Anatomical models were generated with Tetrahedral finite element volumetric meshes generated for these models. Boundary conditions that represent the rest of the cardiovascular system were applied to prepare the models for the computational fluid dynamics simulations. Finite element methods were used to solve the three-dimensional equations governing the dynamics of blood which consist of the momentum balance and mass conservation equations. Simulations focused 
on hyperaemic conditions where the shear rate is high and the blood behaves like a Newtonian fluid: blood was therefore assumed to be an incompressible Newtonian fluid. At measured vessel locations, cross-sectional areas normal to the flow direction were generated and flow rates and pressure values were computed by integrating velocity and pressure components over the cross-sectional areas. hSR was derived using men velocity values instead of APV due to the difficulty of reproducing Doppler velocity measurement. These mean velocity values were computed by dividing integrated flow rates by the cross-sectional areas on the CT-derived anatomical models.

\section{Results}

10 patients met the inclusion criteria for this study. These vessels containing serial CAD within one vessel and had both a prior CT Coronary Angiogram (of sufficient quality for post hoc FFR $_{\mathrm{CT}}$ and non-invasive hSR estimation) and also successful invasive measurements of total vessel and trans-lesional hSR (using a combined Doppler and pressure guidewire). An example case is shown in figure 8.4 .

Mean total vessel FFR was $0.63+/-0.15$ and mean total vessel FFR CT $_{\text {was }} 0.63+/-0.23$. Mean invasive hSR of the 10 serially diseased vessels was $2.67 \mathrm{~mm}+/-2.21 \mathrm{mmHg} . \mathrm{s} / \mathrm{cm}$. Mean hSR as calculated using computed velocity values was $3.19+/-2.65 \mathrm{mmHg} . \mathrm{s} / \mathrm{cm}$. Total non-invasive hSR as calculated using the mean velocity method correlated moderately with invasive hSR $(\mathrm{R}=$ 0.67), See Figure 8.5. 


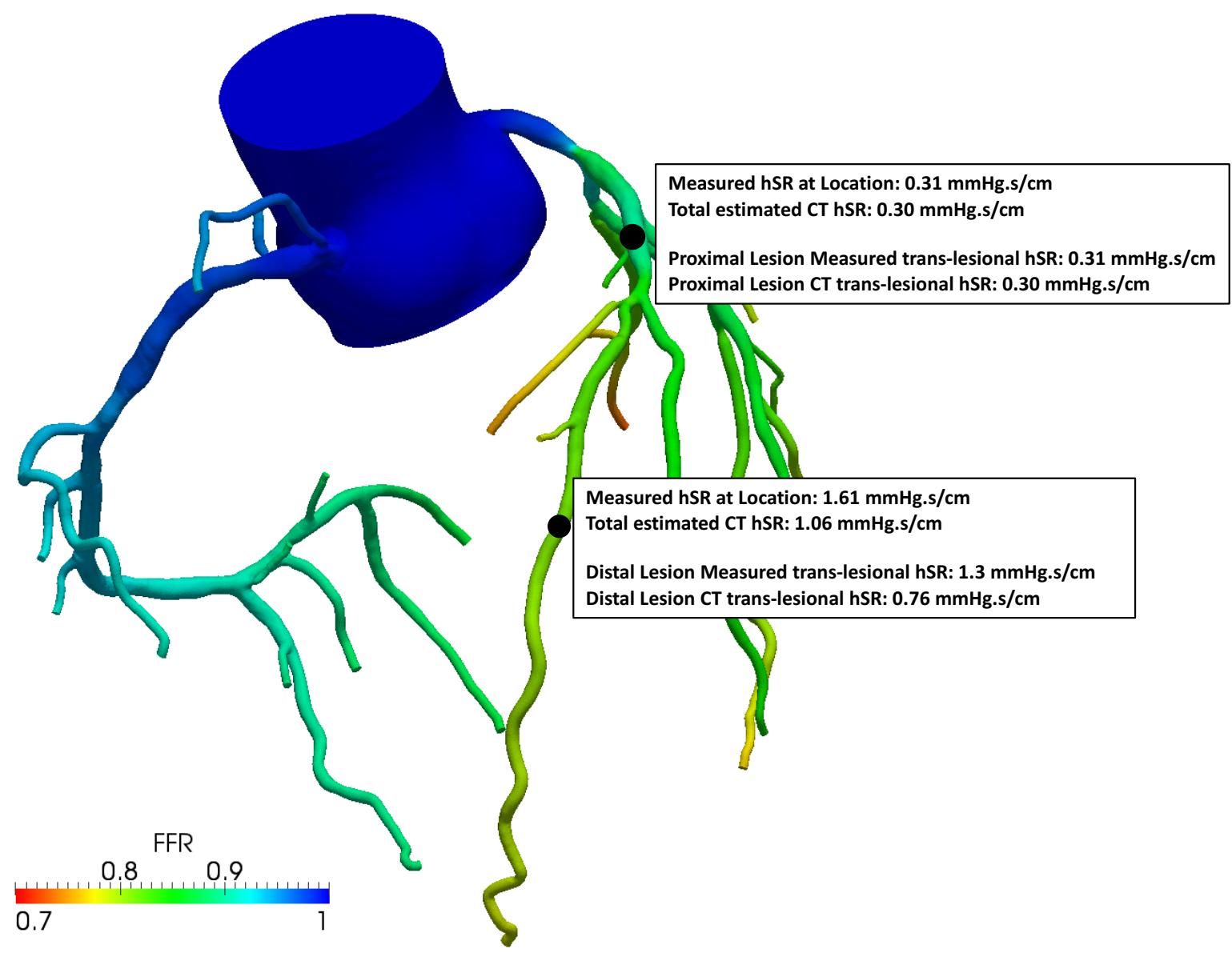

Figure 8.4: Colour-coded FFR $\mathrm{CT}_{\mathrm{T}}$ output showing how trans-lesional CT-hSR values were calculated for each lesion (marked by black spots) and how they were compared to measured invasive hSR values for an example case of a serially diseased LAD artery.

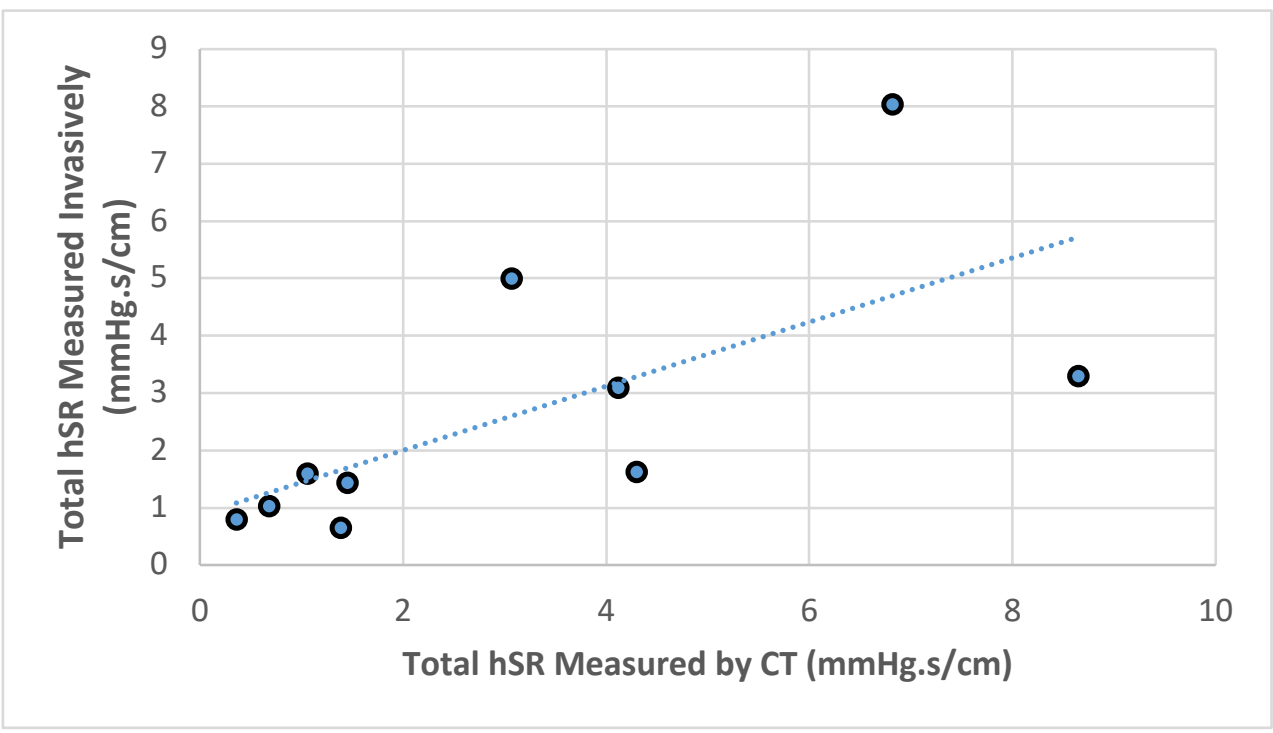

Figure 8.5: Scatter plot showing the correlation of measured invasive total vessel hSR with noninvasive $\mathrm{CT}$ derived $\mathrm{hSR}$ using a mean velocity method $(\mathrm{R}=0.67)$ 
Mean trans-lesional hSR derived invasively was $1.19+/-1.40$. Mean trans-lesional hSR derived non-invasively estimated using CT was $1.59+/-1.51$. Although Trans-lesional hSR values derived invasively showed a trend to correlate with non-invasively estimated hSR, the correlation was poor $(\mathrm{R}=0.28)$, see figure 8.6.

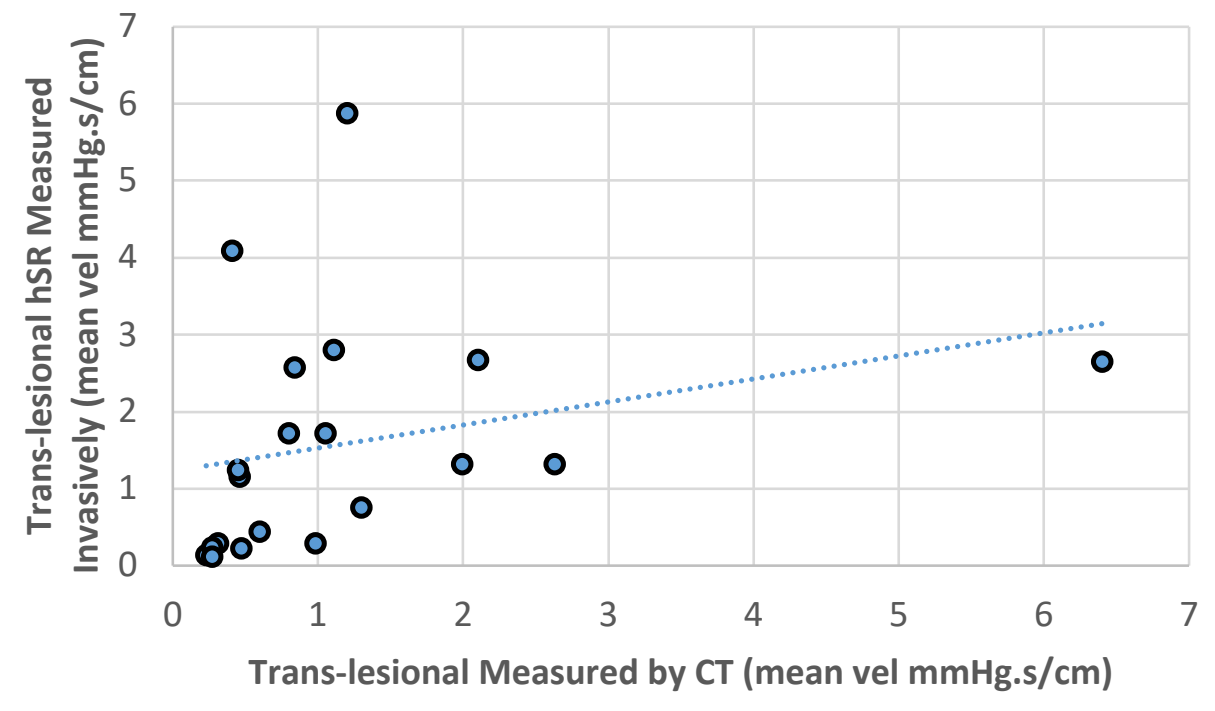

Figure 8.6: Scatter plot showing the correlation trans-lesional hSR measured invasively versus the trans-lesional hSR measured using the novel non-invasive method based on CT-derived mean coronary flow velocity $(\mathrm{R}=0.28)$

\section{Discussion}

In this study we have shown that a non-invasive CT-derived estimation of total vessel hSR correlates moderately with hSR measured invasively using a combined pressure-Doppler guidewire. However, trans-lesional hSR values estimated non-invasively shows weak correlation with hSR measured invasively.

The differences between CT-derived estimation of total vessel hSR and invasively measured hSR can be due to several reasons:

i) There are challenges in identification of true lumen and stenosis geometry as with any CT estimation coronary physiology. 
ii) When computing total vessel hSR, mean velocity values were used instead of Average Peak Velocity (APV) due to the difficulty of reproducing Doppler velocity measurement. Doppler-measured APV values are dependent on the sample volume collected by the transducer and on the location of the transducer: the velocity magnitude can vary significantly from zero near the vessel walls to a maximum velocity value, often near the centre of the lumen. The measurement location can therefore yield very variable results near bifurcation regions or in the regions where the velocity profile shape was not fully developed. In these regions, a small discrepancy in the measurement location can generate big differences between measured and estimated velocity magnitudes.

iii) We computed CT hSR from a mean velocity value by dividing the flow rate by the cross-sectional area with potentially big differences between the measured and estimated hSR values. Differences in APV can therefore be further magnified when computing trans-lesional hSR values as there can also be errors in estimation of cross-sectional area.

The error in estimating trans-lesional values of hSR appears even greater than when assessing hSR on a total vessel basis. This may be a result of variability in effect of computed flow into side branches between lesions and limitations in imaging of diffuse stenoses, particularly those stenoses with complex calcific geometry. This could be avoided by having excellent image quality of the acquired images, avoiding bifurcation regions as reference locations for calculating trans-lesional values, and limiting the measurements to main vessels only.

In summary, when attempting to compare CT-derived trans-lesional hSR values with invasively measurements of trans-lesional hSR values using Doppler, there appears to be a promisingly moderate correlation on a total vessel basis but the correlation of trans-lesional hSR values is far weaker. The errors and poor correlation may arise because of inaccuracies in imaging stenoses, estimating flow velocities and because small side-branching between lesions is difficult to image 
and subsequently compute blood flow for (particularly if uncertainty exists about velocity values near bifurcation regions). As imaging technology and computation improves, improvements can be made by aiming to compute local velocity values nearer precise measurement locations, with the smallest of differences potentially significant. Additionally, improvements can potentially be made by running CFD simulations using pulsatile analyses in order to collect peak velocity values per cardiac cycle to better replicate measured values.

\section{Conclusions}

There is a moderate correlation of total hSR estimated non-invasively to that measured invasively, with a potential to make iterative improvements in the future to this potentially promising index of physiological significance, at least on a per-vessel basis. Currently for serial stenoses, the use of FFR $\mathrm{CT}_{\mathrm{T}}$ and CFD should be restricted to the findings in chapter 8.1 as noninvasive CT estimations of trans-lesional hSR are currently very unreliable and need further development. 


\title{
9. EXPERIMENTAL CHAPTER: CLINICAL UTILITY STUDY
}

\author{
Presented at TCT 2018 (San Diego) \\ In preparation for submission to JACC: Cardiovascular Interventions
}

\section{Acknowledgements}

I would like to pay particular gratitude to the following people for their help with this section:

Prof Divaka Perera (Primary Supervisor)

Dr Ozan Demir (Cardiology SpR)

Dr Kalpa De Silva (Consultant Cardiologist)

Dr Niket Patel (Consultant Cardiologist)

Dr Navin Chandra (Consultant Cardiologist)

Dr Nilesh Pareek (Post CCT Clinical and Research Fellow)

Prof Antonio Colombo (Head of visiting consultants and fellows, Milan) 


\section{Clinical Utility Study Rationale}

A major development from this thesis is that of predicting true stenosis significance by applying a correction equation to a fixed-rate hyperaemic pressure-wire pullback. Our data, described in chapter 7 , suggests this correction equation results in a vast improvement, both in terms of the FFR error across each stenosis and the rate of stenosis misclassification, with results significantly better than other pressure-wire based methods currently used in clinical practice.

Such a solution, to use with routine pressure-wire pullback measurements, is only valuable if it changes clinical decision making and is of clinical utility. The feasibility of an outcome-based trial is questionable as low event rates for stable coronary artery disease mean such a trial would need many thousands of patients to be recruited. Several other measures of clinical utility can however be assessed. They include assessing inter- and intra-observer variability of decision making with and without the novel solution and also assessing the impact this has on resource utilization (revascularisation modality, stent length, stent number). Such a measure of clinical utility can be demonstrated using relatively few patients, as demonstrated by the iFR pullback registry study (utilising data from the multi-national iFR GRADIENT registry) ${ }^{36}$. Accordingly, we have been able to assess the clinical utility of a correction equation together with a smoothed FFR pullback trace. This was done within a randomly selected cohort of 18 serially-diseased vessels. The results of this study are presented in the form of a short stand-alone manuscript (yet to be submitted) in this section.

\section{Introduction}

Physiology-guided revascularisation is growing in popularity, with evidence accumulating over the last decade to show improved clinical outcomes in patients with epicardial coronary artery disease when revascularisation is guided by hyperaemic or resting pressure-derived physiological indices ${ }^{4,10,84,100}$. However, it is unclear whether pressure-derived indices, such as Fractional Flow 
Reserve (FFR) and Instantaneous Wave-Free Ratio (iFR), can reliably assess individual lesions in serial coronary artery disease (CAD) due to the hemodynamic interplay between stenoses ${ }^{86}$.

Pressure-derived physiological indices are all recognised to be prone to haemodynamic interplay between serial stenoses ${ }^{86}$, however, proponents of resting indices have suggested hyperaemic indices, such as FFR, are more prone ${ }^{35}$. This potential error when using FFR in serial CAD led the pioneers of FFR to develop a theoretical solution that involved complex formulae to determine the 'true' FFR contribution of each stenosis by using measurements of intracoronary pressure at various points in the artery following measurement of coronary occlusive pressure after transient balloon occlusion ${ }^{27,28,30}$. The need for transient balloon occlusion prior to committing to a PCI strategy has meant the adoption of these formulae is limited in clinical practice.

Due to this unmet need, we have previously developed and validated a correction equation to use with routine FFR pullback measurements, without the need to measure coronary occlusive pressure $^{101}$ and have shown a significant reduction in error when estimating the true FFR attributable to a stenosis compared to conventional resting and hyperaemic physiological methods ${ }^{102}$. Whilst these in vitro and clinical validation studies have demonstrated a reduction in error and stenosis misclassification when using the novel correction equation, a level of uncertainty remains regarding the clinical utility of such a solution. In this study we therefore assess how the availability of a smoothed FFR pullback trace, together with corrected pressure gradients for each stenosis, alters PCI strategy in real-world clinical scenarios of serial CAD.

\section{Methods}

\section{Study Population}

10 separate attending/consultant operators, working at several European sites, were asked to submit their strategies after presentation of different physiological information for a selection of 
18 elective patients presenting for PCI. In all these cases, at least one vessel had serial CAD, as defined by 2 stenoses of at least 30\% diameter stenosis separated by at least $10 \mathrm{~mm}$, where the operator would consider treating them separately by PCI

\section{Catheter Laboratory Protocol}

All patients included in the cohort of serial CAD had coronary angiography performed via the right radial or femoral artery using a standard Judkins technique. Patients received prior loading with 300mg aspirin and 600mg clopidogrel and intra-arterial heparin to maintain an activated clotting time greater than 250 seconds. Intra-arterial isosorbide dinitrate $(500 \mathrm{mcg})$ was given as standard in all radial-access procedures and intra-coronary isosorbide dinitrate $(500 \mathrm{mcg})$ was administered prior to all pressure wire recordings.

\section{Pressure Wire Pullback Measurements}

Guidewire pressure sensors were normalized in the aorta before being advanced into the distal vessel, with the distal wire position documented fluoroscopically. A resting ratio of distal coronary to aortic pressure $(\mathrm{Pd} / \mathrm{Pa})$ was recorded at this point, following which IV adenosine was commenced to ensure steady state hyperaemia $(140 \mathrm{mcg} / \mathrm{kg} / \mathrm{minute}$, onset of hyperaemia confirmed using invasive pressure waveform changes ${ }^{88}$ ).

Following this, a conventional 'FFR pullback' maneuver was performed, whereby the pressure wire was pulled back at a fixed rate from distal to proximal through the serially diseased vessel. The change in the hyperaemic ratio of $\mathrm{Pd} / \mathrm{Pa}$ across each stenosis within the serially diseased vessel enabled estimation of the attributable FFR of each stenosis (FFR app $)$. Following these measurements, operators were free to manage the patient in keeping with their conventional practice (heart team discussion, PCI using angiographic $+/$ - physiological information or medical management). 
Creating Corrected Smoothed FFR Pullback Information for 'FFRpred'

Raw pressure pullback data (mean Pa and mean Pd during hyperaemia), as presented on standard pressure-wire consoles, were plotted using Matlab software (Mathworks Inc.). In order to assure the quality of the trace, artefactual data points giving non-physiologically high or low values were filtered out and then the trace was plotted using the Smoothing Spline algorithm, which gave a polynomial fit output according to an adjustable smoothing parameter. The output was then assessed for goodness-of-fit. See figure 9.1 for example of a typical FFR pullback output.

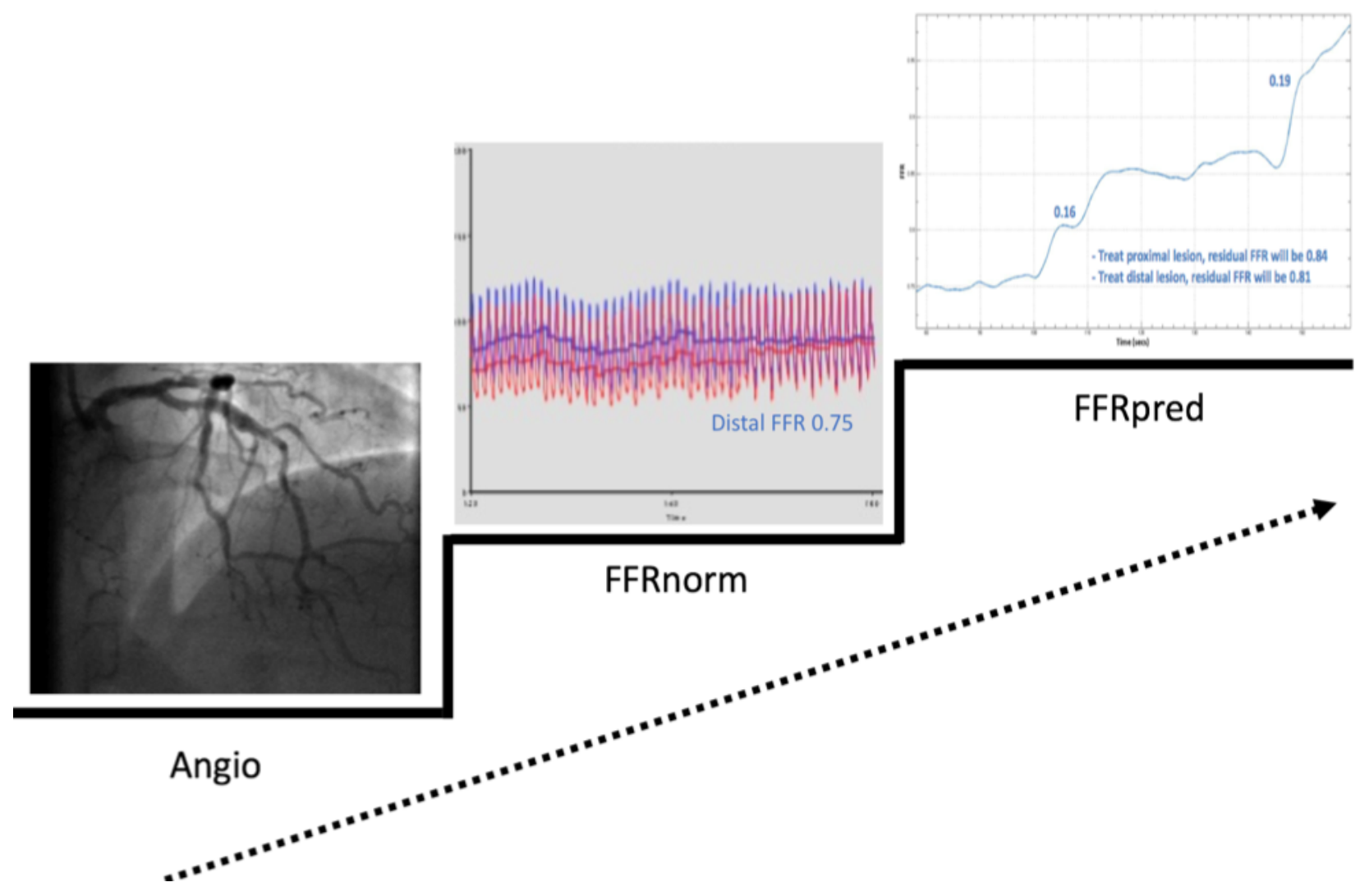

Figure 9.1: Summary of Increasing Information Shown to Operators. 'Angio': Operators asked to submit answers to various questions about the case following information of how the patient presented together with angiographic information only. 'FFRnorm': Operators asked to submit answers following presentation with FFR information as per their conventional practice (FFR in the vessel distal +/- conventional beat-beat FFR pullback data. 'FFRpred':

Overlying this smoothed FFR pullback curve, we presented the operator with the corrected contributions of each stenosis to total vessel FFR (FFRpred). These corrected contributions were derived using our recently developed mathematical solution for serial stenosis interplay, which involves input variables that are confined to data derived solely from pressure-wire pullback ${ }^{103}$. 
This simple and easy-to-use solution assumes a linear relationship between pressure and flow across a stenosis so that the hemodynamic equivalent of Ohm's law can apply, whereby the individual resistance of stenoses and the distal circulation stay fixed regardless of other stenoses being removed. Under these conditions, we have shown that the theoretical FFR can be reliably derived in all configurations of tandem CAD without the need for coronary occlusive pressure, if the variability of collateral flow across intermediate stenoses is assumed to be minimal ${ }^{102}$.

\section{Post Hoc Analysis of Decision Making}

Following the catheter laboratory procedure, 10 operators were shown the following information in sequence for each case (Figure 9.1):

1) 'Angio': Operators were asked to submit answers to various questions about the case following information of how the patient presented together with angiographic information only.

2) 'FFRnorm': Operators were asked to submit answers following presentation with FFR information as per their conventional practice. This usually involved presentation of the FFR in the vessel distal to the serial stenoses or a presentation of the beat-beat FFR pullback data

3) 'FFRpred': Operators were then asked to submit answers after being presented with a smoothed FFR pullback trace with corrected contributions of each stenosis to the total vessel FFR (FFRpred).

With this information, operators were asked to assess whether either of the 2 stenoses were significant or not ('yes', 'no', 'unsure') and also which of the 2 stenoses were more significant (proximal stenosis, 1, or distal stenosis, 2). Operators were then asked about revascularisation strategy with each tier of information (Medical Therapy, CABG or PCI). If PCI was planned, operators were asked about the total stent length and number of stents that would be implanted. 
Statistical analysis was performed using IBM SPSS 24.0 ${ }^{\circledR}$ for Apple Macintosh ${ }^{\circledR}$ (SPSS, Inc., Chicago, IL, USA). Continuous data are presented as mean \pm SD. Categorical data are presented as numbers and percentages and compared using the chi-square test. In order to determine agreement in lesion assessment between observers (inter-observer variability), Fleiss Kappa coefficients were calculated. This coefficient describes the degree of agreement between the ten observers' decisions over that which would be expected by chance. A value of 0 denoted random agreement whilst a coefficient of 1 demonstrated perfect agreement in all cases.

\section{Results}

Eighteen serially diseased vessels were studied, of which 11 were the LAD, 6 involved the LMCA and 1 was the RCA. $78 \%$ (14) of patients were male with a mean age of $64.7 \pm 10.7$ years. The average QCA diameter stenosis was $64.6 \pm 11.2 \%$ and $60.6 \pm 8.9 \%$, for proximal and distal stenoses respectively. Mean distal cumulative FFR of the vessels was $0.72 \pm 0.10 .10$ attending/consultant operators had their decision-making reviewed when presented with different physiological information. All operators worked in high volume tertiary PCI centers and routinely performed FFR measurements for intermediate stenosis assessment as per international guidelines ${ }^{104} .4$ operators worked in London (United Kingdom) and 6 in Milan (Italy).

\section{Operators decision on treatment strategy}

Using angiographic information alone (Angio), operators concluded that 5\% of vessels should receive OMT, $73 \% \mathrm{PCI}$ and $22 \%$ should be treated with CABG. With conventional pressure-wire methods (FFRnorm) these proportions changed to $15 \%, 68 \%$ and $17 \%$ respectively. When presented with the predicted FFR of each stenosis superimposed on a smoothed pullback trace (FFRpred), 17\%, 68\% and 15\% were planned for OMT, PCI and CABG respectively. The agreement about treatment strategy between operators was found to be $0.39,0.64$ and 0.77 with Angio, FFRnorm and FFRpred respectively $(\mathrm{p}<0.001)$. 

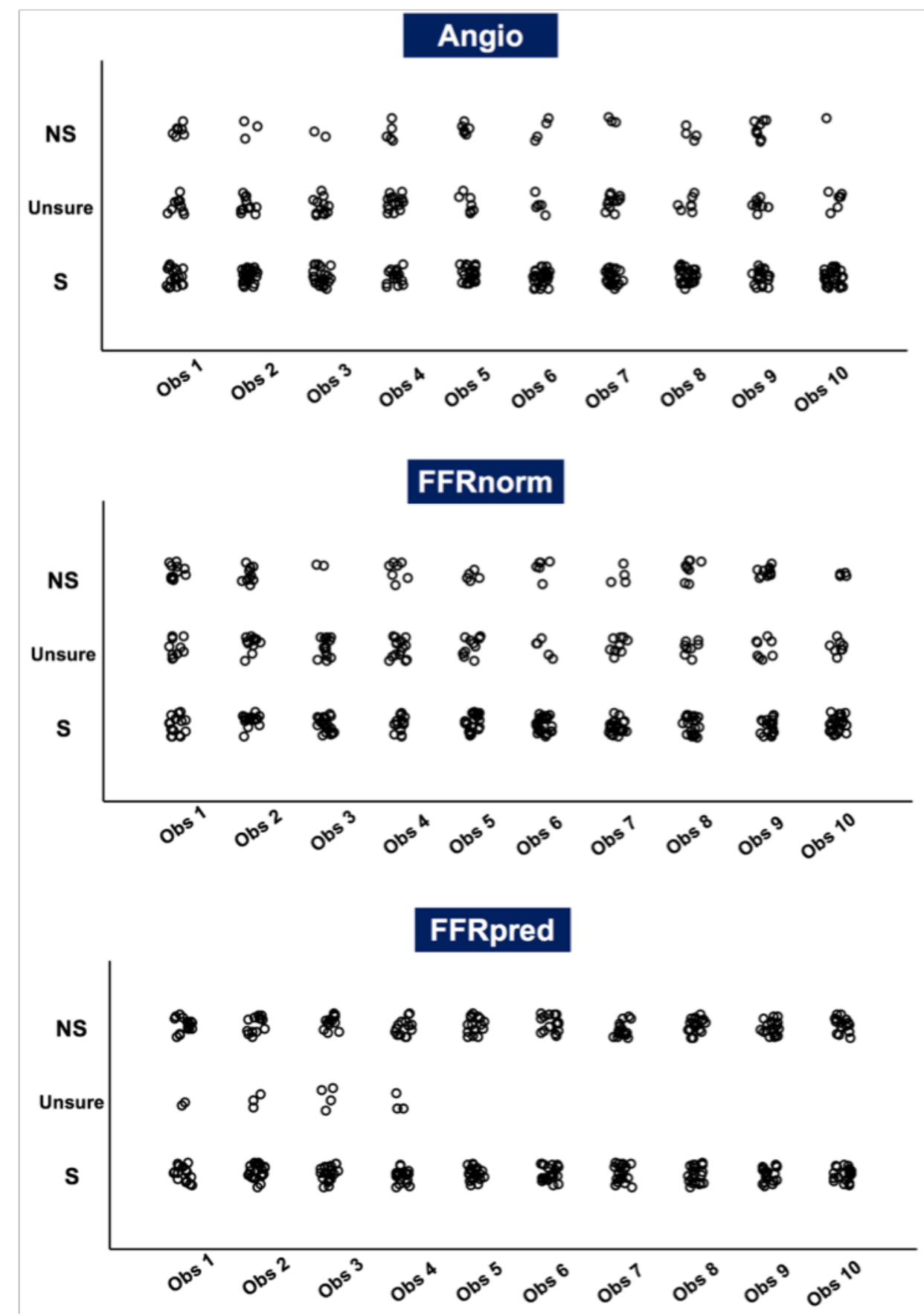

Figure 9.2: Categorized scatter plot demonstrating how certain $(\mathrm{S}=$ significant, Unsure or NS $=$ not significant) each observer felt about the significance of each stenosis when presented with angiographic information only (Angio), conventional FFR measurements (FFRnorm) or predicted FFR of each stenosis superimposed on a smoothed pullback trace (FFRpred).

\section{Operators decision on lesion significance}

When asked about whether a lesion was significant, 26\% were unsure using 'Angio' alone. Supplementing observers with FFRnorm did not significantly affect their uncertainty (28\%). However, when presented with FFRpred, operator uncertainty about the significance of a lesion fell to $3 \%$ of all lesions ( $<<0.001)$, figure 9.2 . 
When asked about which of the two serial stenoses (1, proximal stenosis or 2, distal stenosis) was most significant, the interobserver variability was significantly reduced when presented with FFRpred (k 0.93) versus FFRnorm (k 0.36) or Angio (k 0.35), figure 9.3 and Table 9.1.

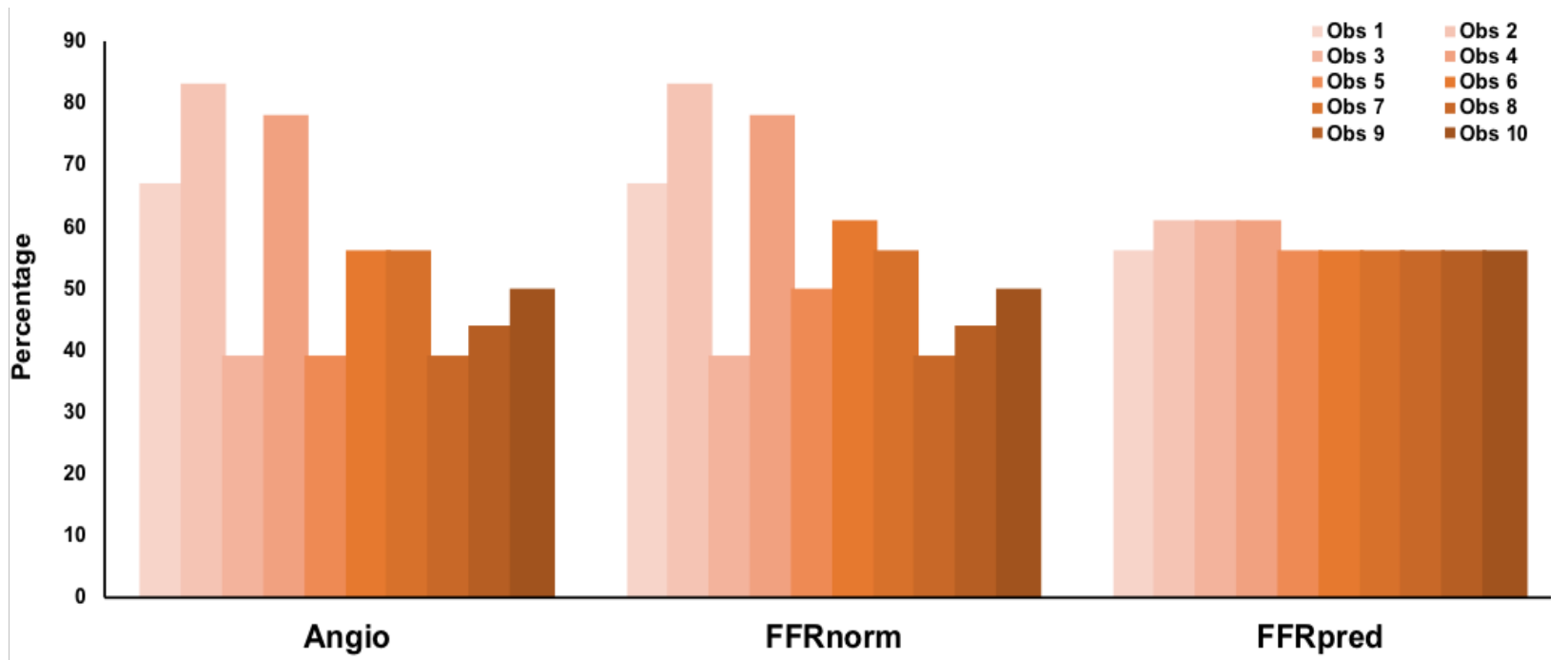

Figure 9.3: Bar charts demonstrating agreement between operators when asked whether lesion 1 is most significant

\begin{tabular}{|c|c|c|c|}
\hline & Angio & FFRnorm & FFRpred \\
\hline $\begin{array}{c}\text { Kappa Value } \\
(\boldsymbol{k})\end{array}$ & $\mathbf{0 . 3 4 5}$ & $\mathbf{0 . 3 5 6}$ & $\mathbf{0 . 9 3 2}$ \\
& $(95 \%$ CI 0.276 to 0.414$)$ & $(95 \%$ CI 0.288 to & (95\% CI 0.863 to 1.00$)$ \\
\hline P Value & $<0.001$ & $0.425)$ & $<0.001$ \\
\hline
\end{tabular}

Table 9.1. Table demonstrating decreasing inter-observer variability in decision on which lesion was most significant, when presented with FFRpred. P values of $<0.05$ used to denote that agreement is significantly different from what would be achieved by chance.

Operators decision on number and length of stents

When operators were asked about the number of stents required, the average stent number per patient was significantly lower at $1.3 \pm 0.5$ stents with FFRpred compared to "Angio" alone $(1.49 \pm 0.57, \mathrm{p}<0.001)$ and FFRnorm $(1.50 \pm 0.57, \mathrm{p}<0.001)$. Moreover, the interobserver 
variability between observers' choices on number of stents to use was significantly reduced when presented with FFRpred (k 0.55) versus FFRnorm (k 0.26) or Angio (k 0.08), $\mathrm{p}<0.001$ (figure 9.4).

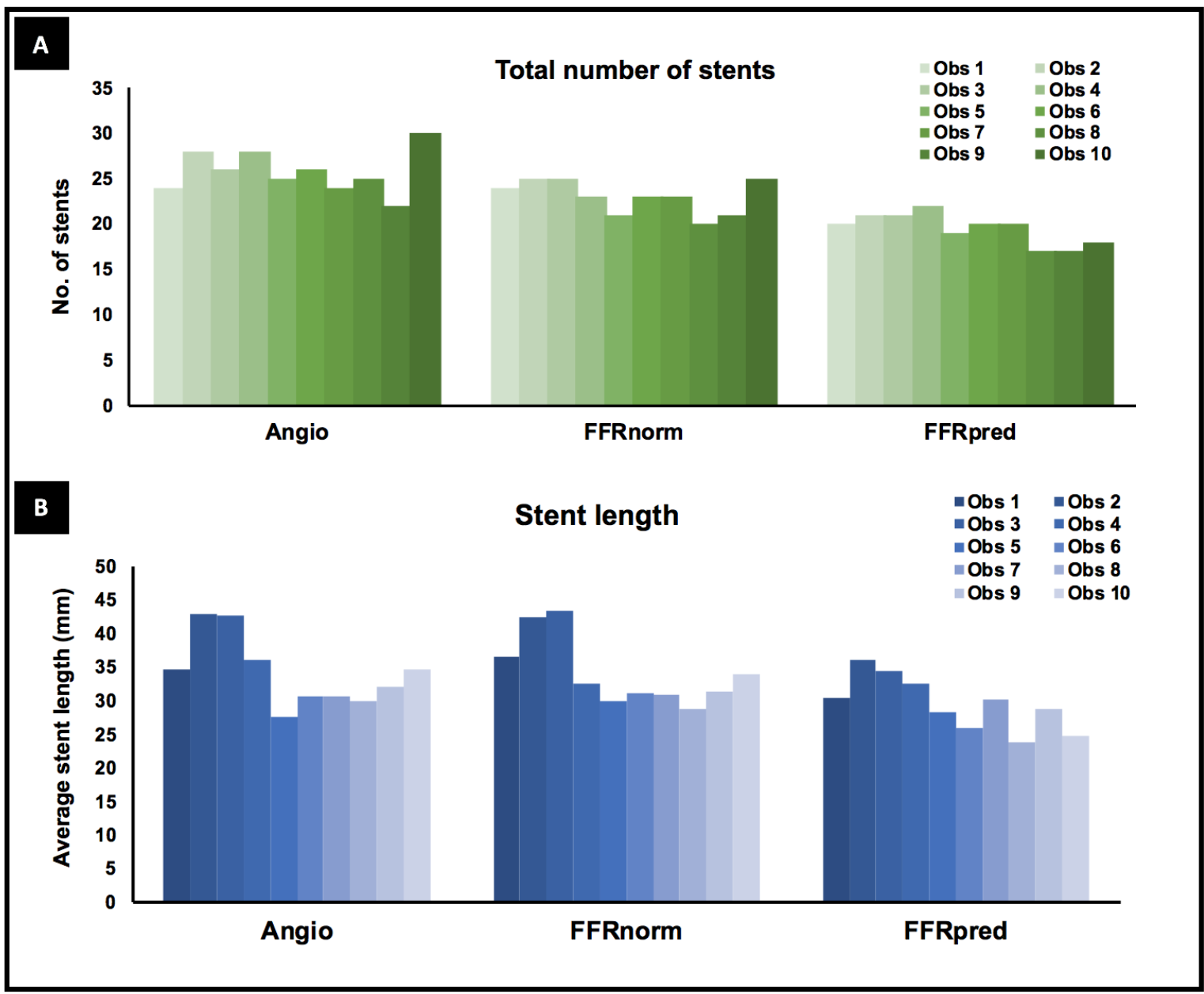

Figure 9.4: (A) Bar Charts demonstrating decreased number of stents with FFRpred compared to FFRnorm or Angio alone. (B) Bar Charts demonstrating total stent length with FFRpred compared to FFRnorm or Angio alone.

When operators where asked about the total length of stent required for treatment of underlying CAD, there was a significant difference between groups. On average, observers opted for shorter total stent length with FFRpred $(29.5 \pm 15.2 \mathrm{~mm})$ compared to FFRnorm $(34.1 \pm 14.4 \mathrm{~mm}, \mathrm{p}<0.001)$ and "Angio" alone (34.6 $\pm 14.3, \mathrm{p}=0.036)$, figure 9.4. Moreover, the interobserver variability was significantly reduced when presented with FFRpred (k 0.93) versus FFRnorm (k 0.85) or Angio (k 0.83), $\mathrm{p}<0.001$. 
Overall, compared to Angio, FFRpred changed decision-making in $68 \%$ of vessels; with a reduction in stent length from $34.6 \pm 14.2 \mathrm{~mm}$ to $29.5 \pm 15.2 \mathrm{~mm}, \mathrm{p}=0.036$. In comparison to conventional 'FFRnorm'-based decision-making, FFRpred changed decision making in $60 \%$ of cases.

\section{Discussion}

In this study we have demonstrated the clinical utility of a smoothed FFR pullback trace, together with corrected pressure gradients for each stenosis and shown how this novel method can alter PCI strategy in real-world clinical scenarios of serial CAD. The main findings of our study to support clinical utility are:

1) FFRpred increased the agreement between operators for treatment strategy in cases of serial coronary artery disease and reduced heterogeneity in decision making

2) FFRpred had a significant impact on the adjudication of stenosis severity, with operators feeling more certain about whether a stenosis required revascularisation and also which stenosis is more significant

3) FFRpred resulted in significantly less stent implantation with a significant reduction in mean stent length and number

We have previously demonstrated that applying this correction equation (FFRpred) to routine FFR pullback data reduces the error in estimating the true FFR contribution of individual lesions and associated misclassification according to an FFR 0.80 threshold for revascularisation ${ }^{101}$. We have also shown a reduction in relative error and misclassification in comparison to resting physiological indices of $\mathrm{iFR}$ and resting $\mathrm{Pd} / \mathrm{Pa}^{102}$. Despite the demonstration of a reduction in error both within our in vitro model and in clinical cases of tandem disease, there remained some doubt regarding whether this would translate to impacting routine clinical decision making. Our study therefore makes a significant advance to previous work done, by showing significant clinical 
utility through reduced ambiguity and increased operator confidence across 10 separate attending operators that were previously naïve to the development of the novel correction method.

This novel solution requires smoothing of conventional FFR pullback data together with incorporation of the correction equation to allow the identification of the true FFR contribution of each stenosis before PCI (figure 9.1). Importantly, for clinical application in PCI planning, FFR pullback can be rapidly performed in nearly all Cardiac Catheter Laboratories and requires no additional hardware. This is important as an element of serial/diffuse CAD is thought to exist in over $25 \%$ of all $\mathrm{CAD}^{5,6}$, meaning the utilisation of such a novel solution should be widespread and easy to adopt. Our FFR pullback-based solution allows the pressure tracing itself to identify the lesions with the greatest influence upon flow impairment, without the need to adopt a new physiological index, use new hardware or measure coronary occlusive pressures. An update to existing pressure-wire consoles would be sufficient to incorporate this solution by displaying a smoothed $\mathrm{Pd} / \mathrm{Pa}$ pullback curve together with the true FFR contribution of a given stent location and the residual FFR in the vessel, were the rest of the disease left alone. Such an update has been trialled on the CoroFlow Coroventis platform. Example of clinical utilisation in a case of serial CAD is shown in figure 9.5 .

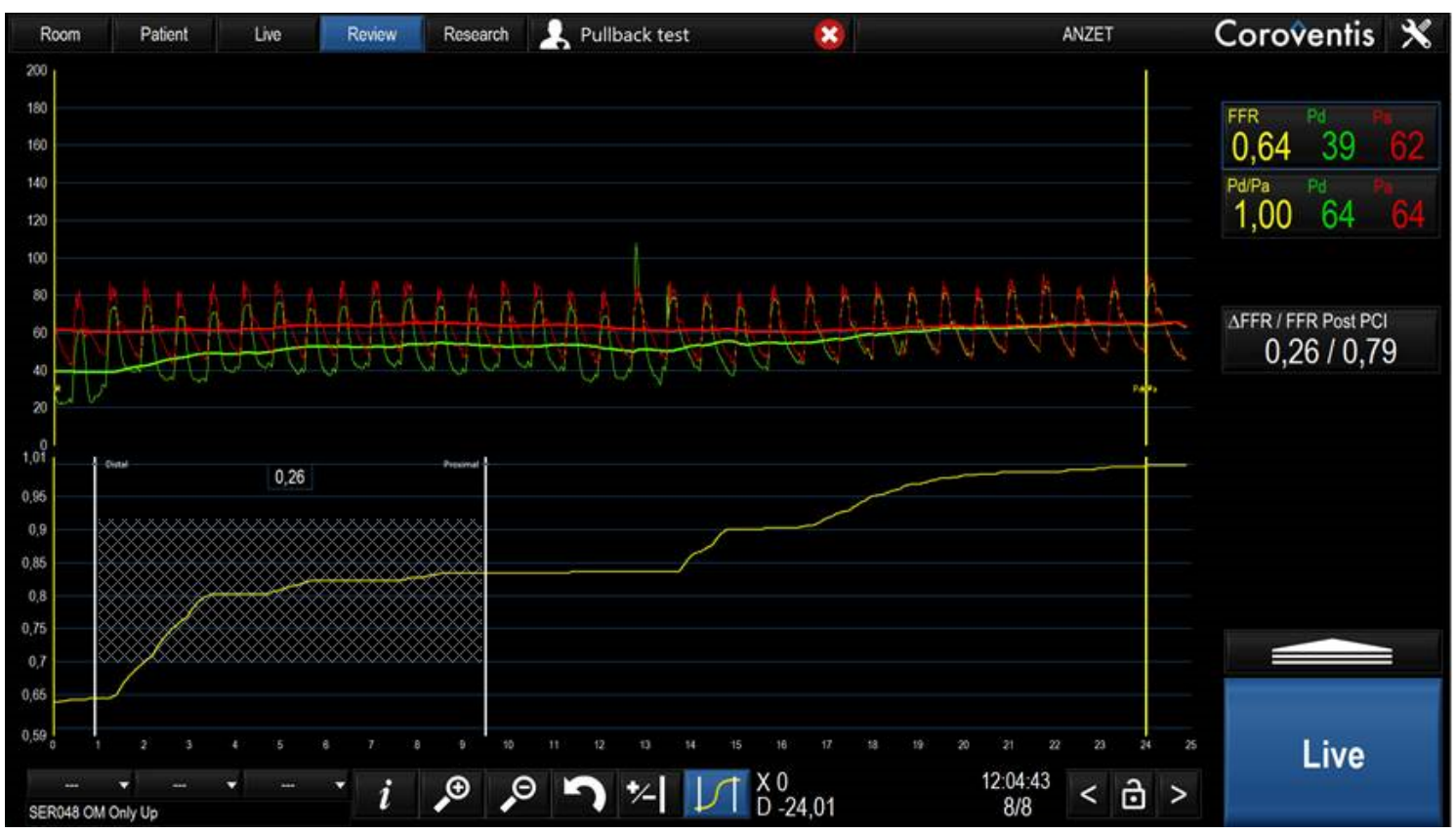


Figure 9.5: Example screenshot from FFR measurement system incorporating delta FFR assessment and calculation of estimated Post PCI FFR result based on localization of distal and proximal stent markers. (CoroFlow, Coroventis Research AB)

This is an important improvement in FFR-methodology, which is likely to enable wider and more accurate adoption of FFR for complex CAD with serial/diffuse disease. Whilst previous iterations of our work have demonstrated a reduction in error versus conventional indices (both resting and hyperaemic), our study goes on to describe significant clinical utility of an even greater magnitude than that described from the iFR gradient registry of iFR pullback in serial CAD ${ }^{105}$. The novel method now needs prospective assessment to see if it improves clinical outcomes and resource utilisation in a larger cohort of multi-centre patients.

\section{Conclusions}

In patients with $\mathrm{CAD}$, hemodynamic interplay between serial lesions is a source of error and stenosis misclassification. Our study shows the clinical utility of a novel method to correct routine FFR pullback outputs by demonstrating increased operator certainty regarding revascularisation and PCI strategy, together with a significant increase in operator agreement. 


\section{SYNTHESIS}

\section{Acknowledgements}

I would like to pay particular gratitude to the following people for their help with this section:

Prof Divaka Perera (Primary Supervisor)

Mr Johan Svanerud (Coroventis AB) 


\subsection{Summary of Findings}

In this thesis I have described the following principle findings:

i) Physiological assessment of serial CAD is prone to significant error, mostly in the form of lesion underestimation, regardless of whether there is a more distal or proximal accompanying stenosis. The extent of this underestimation is proportional to the cumulative burden of disease within the vessel (and therefore inversely proportional to total vessel FFR).

ii) Using an in vitro 3-D printed model of coronary circulation and serial disease, I have shown that total vessel FFR and the change in pressure across a stenosis are the factors which most strongly influence the degree of stenosis interplay during physiological assessment by FFR. Based on these findings, and assuming serial stenoses behave as resistors in series (an assumption we go on to validate in vivo), an easy-to-use mathematical correction equation was created, that significantly reduces this error.

iii) All invasive pressure-derived indices of stenosis severity are prone to significant error in serial CAD. Whilst we found the absolute error is smaller with resting indices such as iFR and resting $\mathrm{Pd} / \mathrm{Pa}$, the misclassification of stenoses due to serial disease interplay was similar, owing to the lower spatial resolution at which these resting indices operate.

iv) Using resistance measurements from simultaneous pressure and Doppler flow measurements, we have demonstrated that serial stenoses behave as resistors in series, with the haemodynamic equivalent of Ohm's Law applicable. Whilst the analogy is imperfect, we have demonstrated that Hyperaemic Stenosis Resistance (hSR) is less prone to the errors and stenosis misclassification seen with conventional pressure-based physiological indices. Widespread utilisation of hSR will however remain limited due to the complexities and variability in making intracoronary Doppler measurements. 
v) Our mathematical correction model, to use with routine hyperaemic pressure-wire pullback measurements, significantly reduces the error of pressure-wire based assessment in serial CAD, to a similar accuracy as the Doppler-derived hSR.

vi) We show that using this novel solution, together with a smoothed $\mathrm{Pd} / \mathrm{Pa}$ pullback trace during hyperaemia, results in increased operator certainty regarding revascularisation and PCI strategy, together with a significant increase in agreement between interventional cardiologists regarding the physiological assessment of individual stenoses. This solution is being incorporated onto a pressure-wire platform (CoroFlow, Coroventis AB) that will enable clinical implementation and further studies.

vii) FFR $_{\mathrm{CT}}$ provides a reliable estimation of the values that would be obtained from routine invasive pressure-wire pullback. In keeping with this, unadjusted trans-lesional $\mathrm{FFR}_{\mathrm{CT}}$ gradients are prone to a similar degree of significant serial stenosis underestimation. I subsequently provide validation for a novel non-invasive $\mathrm{FFR}_{\mathrm{CT}}$-derived PCI planning tool (FFR $\mathrm{FT}_{\mathrm{P}}$ ) and show it to significantly reduce the error of contemporary methods in estimating true stenosis significance within serially diseased vessels (assessed following PCI of the accompanying stenosis). CT/CFD derived estimations of hSR are however still too inaccurate in their present form to provide a reliable method of physiologically assessing serial stenoses. 


\subsection{Proposed Algorithm}

Based on the findings of our catheter laboratory study, the following algorithm (Figure 10.1) can be suggested as a potential interim solution to reduce the error in physiological assessment of serial CAD within the catheter laboratory.

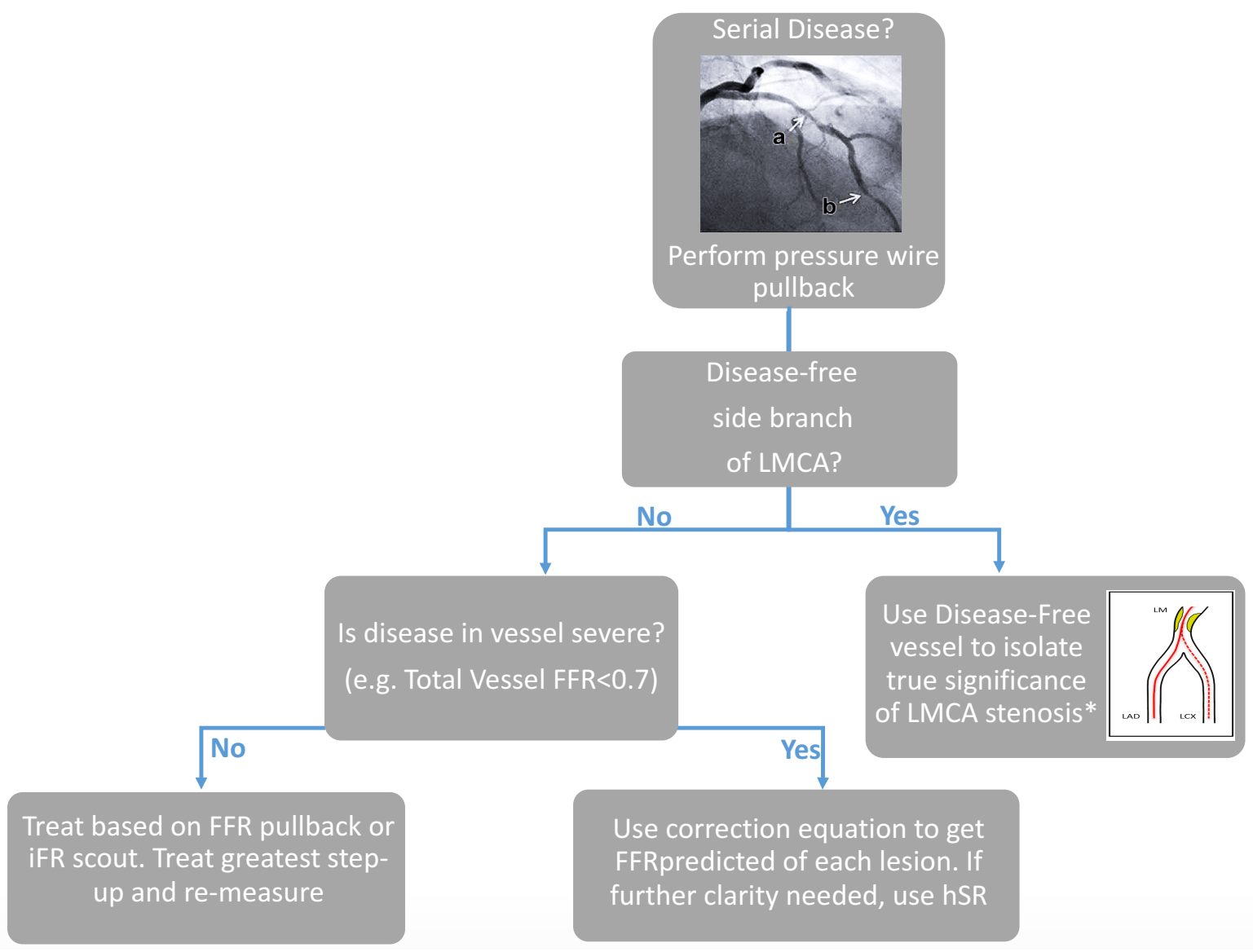

Figure 10.1: Algorithm summarising the findings of this thesis

*Denotes that this part of the recommended algorithm is based on work by Fearon et $\mathrm{al}^{44}$ and is only valid if total vessel FFR of serially diseased branch is $>0.45$

This proposed algorithm is, however, only really appropriate to an academic institute, such as our own, that has access to and experience in using the combined pressure-Doppler based guidewires. A truly clinically useful algorithm would be based on simply the use of either a pressure-based physiological index, such as that demonstrated with our theoretically-derived correction equation (FFRpred), or one that allows accurate non-invasive prediction of residual stenosis significance after PCI, such as that demonstrated in our validation of the encouraging FFR $_{\text {CT }}$ PCI Planning tool (FFR 
become established and fully implemented, the catheter laboratory algorithm described in figure 10.1 is a reasonable, albeit imperfect, strategy for now.

\subsection{Resting Versus Hyperaemic Indices?}

Despite the wealth of guidelines and evidence supporting FFR-guided revascularisation, less than $10 \%$ of eligible cases have FFR assessment ${ }^{106}$. This has led many to question whether the need for adenosine-induced hyperaemia is a hindrance, with resting invasive indices, particular iFR, growing in popularity.

Some have suggested the resting index of iFR is more cost-effective (often citing the saving on the cost of adenosine), results in shorter procedure times and is less uncomfortable for the patient ${ }^{107}$. Based on this, proponents of the resting physiological indices have just had to argue the case for 'non-inferiority' in outcomes versus FFR-guided revascularisation with some recent trial data for iFR-guided revascularisation supportive of this standpoint ${ }^{11,12}$. In parallel to the evidence supporting $\mathrm{iFR}$, data is growing to suggest that the resting ratio of $\mathrm{Pd} / \mathrm{Pa}$ is almost identical to $\mathrm{iFR}^{16,17}$ and therefore a $\mathrm{Pd} / \mathrm{Pa}$ revascularisation versus FFR guided revascularisation trial may also yield non-inferior results. This is despite ongoing debate regarding whether resting indices provide the same information about stenosis severity as FFR, with studies such as our own Thresholds study (see Appendix) suggesting there may be a difference between resting and hyperemic indices. Whether or not this is the case, it is clear that we are experiencing a rebirth of resting physiological indices and novel indices such as iFR are only driving the amount of physiologically-guided revascularisation we are performing, regardless of the modality.

One of the few areas where resting indices have claimed 'superiority' (not just 'non inferiority'), over hyperaemic assessment, is in the assessment of serial CAD. This has been predominately driven by small studies showing a relatively small difference between expected iFR and 
observed iFR from a straightforward iFR pullback manoeuvre ${ }^{35}$. Following this, in recent months a clinical utility study of iFR pullback has been published that lends support its clinical utility $^{36}$. Despite this, prior to this thesis, there still lacked evidence to support this claim of 'superiority' of resting indices versus hyperaemic resting indices in physiological assessment of serial CAD.

Our study represents the first study to compare how various invasive physiological indices compare against each other in the assessment of serial CAD. We show that despite a small reduction in the error with resting indices, the overall message is that all pressure-derived physiological invasive indices are prone to significant errors. Whilst there may be some theoretical reduction in serial stenosis interplay in the resting state because of intact autoregulatory mechanisms, this is counter-balanced by the fact that iFR and PdPa operate at a lower spatial resolution (each stenosis is associated with a smaller pressure gradient at rest) and therefore any errors are amplified.

\subsection{Future Directions}

When it comes to physiological assessment of serial CAD, there are several areas of development that will affect future directions when it comes to the clinical implementation of an optimal solution. These are areas I hope to continue being involved with whilst completing my clinical training:

i) Physiological-Anatomical Co-registration: Whichever physiological index is used, one of the major unmet needs is being able to marry the pullback trace to the angiogram. Recently, there has therefore been an increasing drive to co-register physiological data with anatomy, with the developers of iFR pullback (Philips Volcano) leading this field (see figure 10.2). 


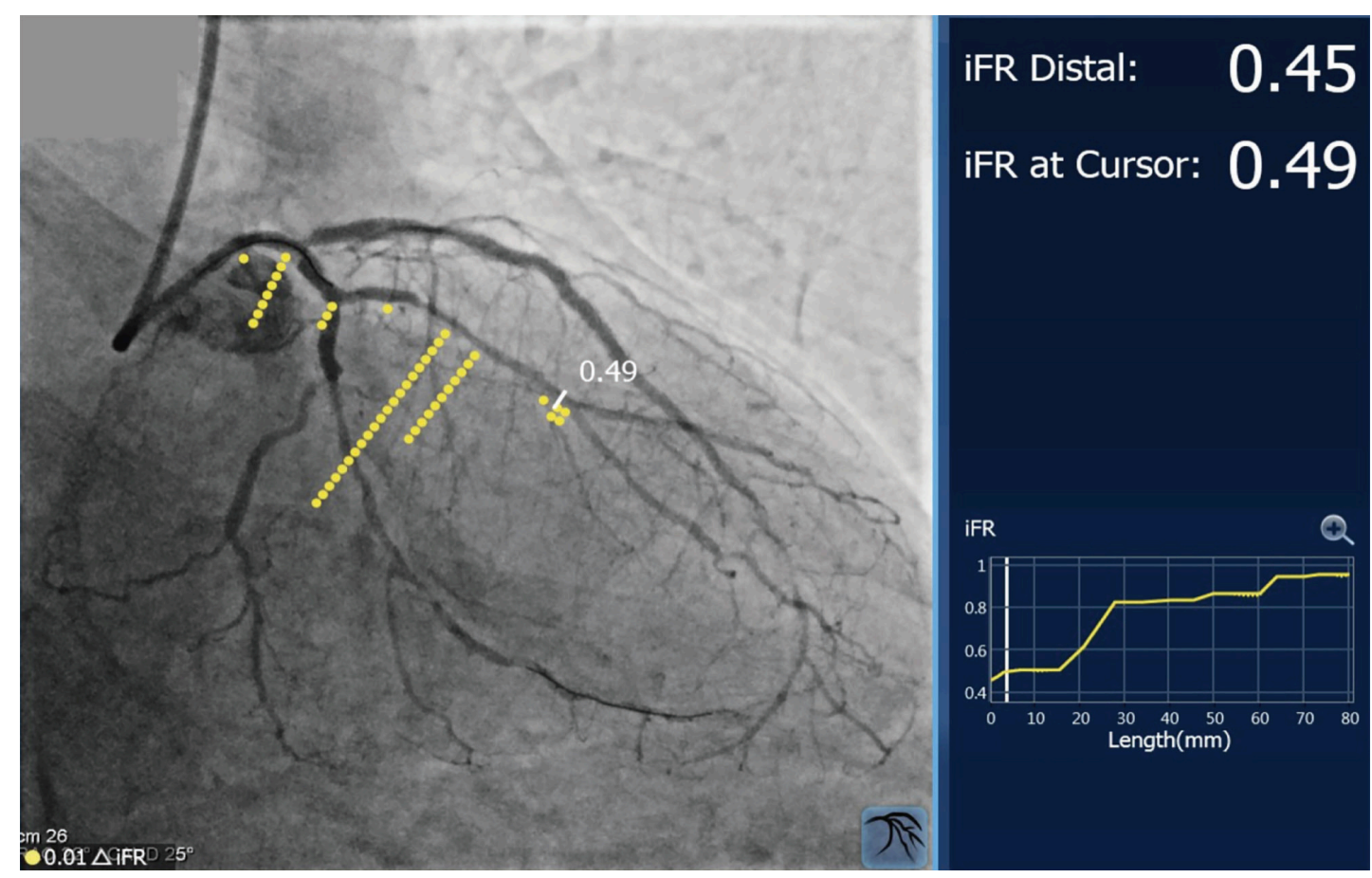

Figure 10.2: Example of physiological coregistration with the coronary angiogram. An instantaneous wave-free ratio (iFR) pullback was performed, and using a commercially available system (SyncVision, Philips), the pullback is coregistered on the coronary angiogram. Each yellow dot represents a change in iFR of 0.01 , with the graphic pullback depicted on the right of the figure. The distal iFR at the cursor point is 0.49 . The cursor can be moved to assess the iFR at any point along the pullback, allowing the operator to assess the drop in iFR across serial stenoses. (Taken from Modi et al, JAMA Card 201892)

Regardless of whether or not our novel solution (figure 9.5) grows in popularity, any physiological PCI planning tool will ultimately need to be incorporated into such a coregistration solution, such as the one being developed by Philips Volcano. One of the leading vendors of FFR (Abbott) has recently developed co-registration capabilities using Optical Coherence Tomography (OCT), rather than overlaying physiological information onto the angiogram. Adding intravascular imaging and 'true' physiological data to an angiogram would represent the ultimate solution and not only inform us whether to treat a stenosis but exactly how (stent length, diameter etc).

\section{ii) Computational Fluid Dynamics and Non-Invasive Estimations of FFR:}

Despite the growing evidence supporting physiology-guided management of stable CAD, it is used in only a fraction of eligible patients ${ }^{106}$. Some of the reasons cited include perceived 
risk/burden to the patient, increased procedural cost and increased procedural time. In addition, as described in chapter 8 , evidence suggests over $50 \%$ of patients referred for invasive coronary angiography have no significant disease ${ }^{61}$. As a result, there has been a quest for establishing non-invasive estimations of FFR either from upstream imaging modalities (e.g. FFR $\mathrm{FT}_{\mathrm{CT}}$ ) or recently in the form of deriving FFR exclusively from diagnostic angiography (e.g. Quantitative Flow Ratio, QFR 108 109).

These solutions, as described earlier, rely on principles of CFD being used in combination with knowledge of precise lesion geometry. Such methods may have an inherent benefit in the assessment of the true significance of individual stenoses within a serially diseased vessel, as

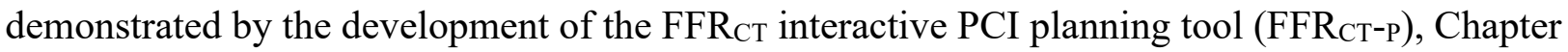
8.1. The results of our study suggest using methods, such as the $\mathrm{FFR}_{\mathrm{CT}-\mathrm{P}}$ tool, can aid more accurate prediction of true stenosis significance of individual stenosis in serial CAD compared to contemporary pressure-wire pullback and conventional FFR $\mathrm{FT}_{\mathrm{C}}$ outputs. Further studies are necessary in a larger number of patients to demonstrate the clinical utility of this non-invasive index. If clinical utility is demonstrated, this tool can be used in all patients to plan PCI and can even be displayed on monitors within catheter laboratories when choosing whether to stent a stenosis, stent position and stent length.

iii) More Stringent Thresholds to Define Ischaemia and Significant Stable CAD:

Growing evidence suggests the benefits of revascularisation seem to be greatest in those with a high burden of ischaemia ${ }^{7,110}$. At the same time, the last decade has seen mounting evidence to suggest medically managed stable CAD is associated with a good prognosis and more modest symptomatic benefit than originally envisaged. As a result, there is now a growing question about whether the physiological thresholds we use to guide revascularisation are suboptimal. We address this issue in appendix A: 'Revisiting the Optimal FFR and iFR Thresholds for Detecting Significant Coronary Artery Disease'. While the findings of this additional study don't change the findings of this thesis, it may mean fewer lesions (based on their 'true' FFR/iFR) may be 
appropriate for revascularisation. Rightly or wrongly, this may mean less of a tendency to revascularise stable $\mathrm{CAD}$, regardless of physiology.

iv) Physiological Assessment of LMCA Disease: As described in section 5.5, the issue of downstream serial disease if one of the major reasons why LMCA disease has been largely excluded from trials of physiology-guided revascularisation. The problem of stenosis underestimation with serial disease may mean misclassifying a potentially significant LMCA stenosis when it is actually significant.

Whilst the proposed algorithm described in section 5.6 is of value where there is a disease free daughter vessel, LMCA disease is commonly found with downstream serial disease in both main branches. The findings of this thesis will enable operators to feel more confident about assessing the true physiological significance of LMCA disease, regardless of whether there is accompanying disease present in downstream vessels. This will hopefully pave the way to doing a trial of physiology-guided revascularisation that finally includes LMCA disease in sufficient numbers to form a meaningful conclusion.

\subsection{Limitations of No 'Gold Standard' For Invasive Physiological Assessment}

Physiological assessment and ischaemia testing have long been plagued by the lack of a 'gold standard' against which to compare. FFR and hSR were validated against non-invasive tests ${ }^{8}$, iFR against $\mathrm{FFR}^{73}$ and now we have come full-circle with non-invasive tests being validated against FFR ${ }^{111}$. The lack of reference standard means the thresholds used for these indices is also debated (see our study in appendix A).

Establishing a gold standard of physiologically assessing the significance of an individual lesion is similarly challenging. In this thesis, I have shown within the wet laboratory in vitro experiments, that lesion isolation is possible by replacing a serially diseased 3D printed phantom 
with the corresponding discretely 3D printed phantom - providing us with a 'gold' reference standard against which to assess apparent FFR and corrected FFR. Using the results of this work we were also able to create a solution and establish some basic principles.

Comparing various physiological indices and the correction equation in the catheter laboratory against a 'reference standard' is however more of a challenge. The two methods used to isolate a lesion (both methods were used in chapter 7) are described below, together with their strengths and flaws:

i) Measurements of apparent and true lesion significance can be made within the serially diseased vessel before and after PCI of an accompanying stenosis. This method reflects the question many clinicians want answering: once I perform PCI of stenosis ' $\mathrm{X}$ ', what will be significance of stenosis ' $\mathrm{Y}$ '? Wherever possible, this method of stenosis isolation was used as the reference 'gold standard'. However, the validity of physiological indices and the dynamic state of microvasculature immediately post PCI is occasionally questioned with limited data to verify post PCI physiological measurements. The validity of post-PCI physiological measurements is certainly an area that needs further validation work in the future.

ii) In the case of a large serially diseased vessel with a disease free branch vessel between the serial stenoses, this disease-free branch can be used to isolate the true significance of the proximal stenosis. Whilst this method has theoretical flaws, based on the inconsistent volume of subtended myocardium, previous data suggests it is reliable method of assessing the $\Delta \mathrm{FFR}_{\text {true }}$ of the LMCA stenosis. This method was initially validated in animal studies ${ }^{41,45,46}$. Fearon et al then demonstrated this in a clinical study of 91 LMCA lesions following PCI of the LAD, LCx, or both. FFR was measured in the LAD and LCx before and after creation of downstream stenoses using balloons within newly placed stents and measuring FFR in the disease-free vessel. They then compared the true LMCA FFR measured in the disease-free vessel to the apparent FFR measured in the stenosed vessel and found the numerical difference was not deemed clinically 
significant. They concluded that in most cases, downstream disease does not significantly impact LMCA FFR when the pressure wire is positioned in the disease-free vessel and using a disease-free daughter vessel provides a fairly accurate assessment of the true significance of the proximal (LMCA) stenosis ${ }^{44}$.

Whilst both these methods of isolating the true significance of a stenosis in vivo have potential flaws, they both represent the best available reference standard against which to compare different physiological indices and novel solutions for serial CAD.

\subsection{Conclusions}

In this thesis, we have shown that physiological assessment of stenoses in the presence of serial CAD is prone to significant underestimation, proportional to the cumulative burden of disease within the vessel. All pressure-derived physiological indices, resting and hyperaemic, are prone to error and stenosis misclassification. Doppler-based resistance indices are less prone to this error but utilisation is limited by difficulties in obtaining Doppler traces. The finding that serial stenoses generally behave as resistors in series does however support the use an FFR correction equation, derived using our 3D-printed in vitro study, that does not require measurement of coronary occlusive pressure. Applying such a correction equation to routine pressure wire pullback measurements significantly reduces the error in estimating true stenosis significance and we have shown it to have significant clinical utility.

In this thesis I also describe the development and validation of a new non-invasive technique to optimise the physiological assessment of serial CAD. This non-invasive FFR $_{\mathrm{CT}}$-derived PCI planning tool can aid more accurate prediction of true stenosis significance in serial CAD, in comparison to contemporary invasive and non-invasive methods. 
The next step will be to establish multi-centre clinical utility studies of these novel invasive and non-invasive physiological methods. Eventually, the solutions will need to be co-registered with coronary anatomy and tested in the important scenario of LMCA disease, which is frequently found with downstream serial CAD. 


\section{APPENDICES}




\section{APPENDIX A: REFINING THE ASSESSMENT OF STENOSIS SEVERITY IN CLINICAL PRACTICE}

\section{Peer-Reviewed Publications Relating to This Section:}

1. Modi BN, Rahman H, Sherif SA, Ellis H, Eruslanova K, Chiribiri A, Perera D. Is heart rate response a reliable marker of adenosine-induced coronary hyperaemia? Int $J$ Cardiovasc Imaging (2018)2018. doi:10.1007/s10554-018-1309

2. Modi BN, Rahman H, Kaier TE, Ryan M, Williams R, Briceno N, Ellis H, Pavlidis A, Redwood S, Clapp B, Perera D. Revisiting the Optimal FFR and iFR Thresholds for Detecting Significant Coronary Artery Disease. Circulation: Cardiovascular Interventions 2018;11:e07041. DOI: 10.1161/CIRCINTERVENTIONS.118.007041 


\section{A1: COMPARING THE DIAGNOSTIC ACCURACY OF FFR AND IFR: IS IT TIME TO CHANGE TREATMENT THRESHOLDS?}

\section{Abstract}

Background: Fractional Flow Reserve (FFR) and Instantaneous Wave-Free Ratio (iFR) are the most widely used invasive tests to guide management of stable coronary artery disease (CAD). There has been a gradual 'upward creep' of revascularisation thresholds for both FFR and iFR, compared to initially established thresholds. The resulting increase in revascularisation is at odds with increasing evidence of the lack of prognostic benefit of revascularisation in stable CAD. Objectives: Using an independent invasive reference standard, this study aimed to a) define the optimal diagnostic thresholds for FFR and iFR and b) to compare the performance of iFR, FFR and resting $\mathrm{Pd} / \mathrm{Pa}$ at detecting functionally significant coronary disease. Methods: Distal coronary $(\mathrm{Pd})$ and aortic pressure $(\mathrm{Pa})$ were measured in 75 patients undergoing coronary angiography +/- PCI with resting Pd/Pa, iFR and FFR calculated as previously described. Doppler flow velocity was simultaneously measured and hyperaemic stenosis resistance calculated as $\mathrm{hSR}=\mathrm{Pa}-\mathrm{Pd} / \mathrm{APV}$ (where $\mathrm{APV}=$ average peak velocity). $\mathrm{hSR}>0.80 \mathrm{mmHg} . \mathrm{cm}^{-1} . \mathrm{s}$ was used as an invasive reference standard for stenosis significance. Results: An FFR threshold of 0.75 had the best diagnostic accuracy (84\%) whereas for iFR the optimum threshold was 0.86 (76\% accuracy). At these thresholds, the discordance in classification between indices was $11 \%$. The accuracy of contemporary thresholds ( 0.80 for FFR and 0.89 for iFR) was significantly lower $(78.7 \%$ and $65.3 \%$ respectively for FFR and iFR) with a $25 \%$ rate of discordance between indices. The optimal threshold for $\mathrm{Pd} / \mathrm{Pa}$ was 0.88 , which gave an accuracy of $77.3 \%$. When comparing the 3 indices at their optimal thresholds, FFR had the best diagnostic performance (area under the curve $0.91 \mathrm{FFR}$ vs. $0.79 \mathrm{iFR}$ and $0.77 \mathrm{Pd} / \mathrm{Pa}, \mathrm{p}=0.002$ ).

Conclusions: Contemporary FFR and iFR thresholds provide suboptimal diagnostic accuracy compared to a FFR threshold of 0.75 and an iFR threshold of 0.86 , which are also the thresholds in the original derivation studies. Whether the use of the more rigorous thresholds would result in selection of a population gaining greater symptom and prognostic benefit needs assessing in 
future trials of physiology-guided revascularisation.

\section{Condensed Abstract:}

In recent times, there is growing acknowledgement that revascularisation for stable CAD should be restricted to patients with functionally significant CAD. FFR and iFR, which have become cornerstones of determining functional significance, have seen an 'upward creep' in thresholds over the last 2 decades, with subsequently more revascularisation. In this study, we have determined the optimal threshold for each index, using an independent physiological reference standard, to show that contemporary thresholds may have suboptimal diagnostic accuracy to those originally described.

\section{Introduction}

The benefit of revascularisation in stable coronary artery disease (CAD) is a contentious issue, with mounting evidence suggesting that patients on optimal medical therapy alone have an excellent prognosis with significantly improved symptoms, as suggested by the COURAGE trial $^{112}$ and recently the Sham-controlled ORBITA trial ${ }^{113}$. On the other hand, we have evidence suggesting physiology-guided revascularisation, whether it be in the form of Fractional Flow Reserve (FFR) $)^{3,4,74}$ or Instantaneous Wave Free Ratio (iFR $)^{11,12}$, is associated with significantly improved symptoms and patient outcomes, compared to revascularisation based on angiographic appearances alone. The benefits of revascularisation seem to be greatest in those with a high burden of ischaemia ${ }^{7,110}$.

The initial derivation studies of FFR were performed against a combination of non-invasive tests including SPECT and stress echocardiography and showed an optimum threshold of $0.75^{8,74}$. Following this, subsequent evidence of improved clinical outcomes (driven by a reduction in repeat or urgent revascularisation) was demonstrated in the FAME and FAME 2 trials ${ }^{3,4}$, using the higher threshold of FFR $\leq 0.80$, to provide clinicians with the "safety net" of improved negative predictive value. FFR $\leq 0.80$ has gained such wide acceptance as the dichotomous 
threshold to detect significant coronary disease that many novel physiological indices, including some non-invasive measures ${ }^{111}$, have been validated against it. The invasive physiological index of iFR was also originally derived and validated against an FFR $\leq 0.80$ threshold. The ADVISE and CLARIFY studies suggested that iFR thresholds of 0.83 and 0.86 provided optimal agreement with the FFR threshold of 0.8. Subsequent studies demonstrated discordance of up to $40 \%$ between FFR and $\mathrm{iFR}^{16}$, and an iFR grey-zone of $0.86-0.93$ was suggested, whereby cases in this range subsequently have FFR-guided management, with an improvement in concordance with FFR ${ }^{114}$. A drive for a discrete threshold led to further analyses of discordance ${ }^{15,115}$ and eventual adoption of a higher iFR threshold of $\leq 0.89$. Management of stable CAD using FFR $\leq 0.80$ or $\mathrm{iFR} \leq 0.89$ has been shown to result in equivalent outcomes in recent trials in patients who generally have a good prognosis ${ }^{11,12}$. Whether the loss of specificity inherent in this 'upward creep' of the diagnostic FFR and iFR thresholds results in patients being inappropriately revascularised, in the absence of a substrate for ischaemia, remains unclear.

This 'upward creep' in thresholds has resulted in a 'physiological greyzone' between the FFR 0.75 and 0.8 thresholds. Recent data from the IRIS-FFR registry by Kang et al has suggested coronary revascularisation of greyzone-FFR cases is not associated with improved outcomes ${ }^{116}$. This 'greyzone' has also existed for iFR, $(0.86$ - 0.93) with a hybrid iFR-FFR guided strategy sometimes used to resolve the uncertainty ${ }^{114}$. However, recent randomized trials have used a single rule-in and rule-out iFR threshold of $0.89^{11,12}$.

This study aimed to assess the optimal diagnostic thresholds for these invasive physiological indices against an independent reference standard and in doing so, sought to also compare the diagnostic accuracy of these indices at both the contemporary and optimal thresholds. In the absence of a true ischaemic gold standard, several noninvasive imaging methods have previously been used to further evaluate invasive physiological methods, with the major hurdle being these methods only isolate ischaemia in a myocardial territory rather than a specific vessel. In this study we therefore used Hyperaemic Stenosis Resistance (hSR) as an invasive reference standard of physiological significance. hSR is an invasive index calculated by 
measurement of both intra-coronary pressure and Doppler flow velocity and so overcomes many of the limitations of flow-only and pressure-only based indices ${ }^{21,42,99}$. It is recognized as being more stenosis specific, having previously been used as an invasive reference standard to compare pressure-derived indices, for example in the CLARIFY study ${ }^{87}$. It is considered to be independent of resting or hyperaemic conditions within the vesse ${ }^{87}$.

\section{Methods}

\section{Study Population}

Patients who were scheduled to undergo coronary angiography with a view to proceeding to PCI for suspected or confirmed stable ischaemic heart disease were eligible for inclusion. Exclusion criteria were significant valvular heart disease, an unstable coronary presentation (myocardial in the prior 4 weeks or CCS IV angina), coronary disease that was not suitable for instrumentation (as below), previous $\mathrm{CABG}$ and contraindications to pharmacological hyperaemia with adenosine. Study protocols were approved by the UK national research ethics committee and all patients provided written consent.

\section{Cardiac Catheterization}

Dual antiplatelet therapy was initiated in all participants prior to the procedure. Procedures were performed via the right radial artery or right femoral artery using 5 or $6 \mathrm{Fr}$ guiding catheters. Bolus unfractionated heparin was administered to maintain an activated clotting time $>250$ seconds. Following diagnostic coronary angiography intracoronary nitroglycerin was administered and a 0.014" dual pressure-Doppler sensor guide wire (ComboWire, VolcanoPhilips, California) was calibrated to aortic pressure and the tip delivered to the distal epicardial vessel. Aortic pressure was measured via the fluid-filled guide catheter. The Doppler signal was optimized and recording commenced. Hyperaemia was achieved using an intravenous (IV) infusion (dose $140 \mathrm{mcg} / \mathrm{kg} / \mathrm{min}$ ) or intracoronary (IC) boluses (dose $60 \mathrm{mcg}$ repeated three times) 
89. After completion of the infusion or each intracoronary bolus, we waited for baseline hemodynamic parameters (Heart Rate, Blood Pressure and Doppler Flow velocity) to return to baseline before the next measurement. Offline calculation was subsequently performed of Resting Pd/Pa, FFR, iFR, and hSR using Cardiac Waves Software (Kings College London, London, United Kingdom). Coronary angioplasty was then performed if indicated.

\section{Data Recording and Analysis}

Pressure data: For Pd/Pa and iFR, the lowest 5 beat average obtained was recorded with all analyses performed in a fully automated manner without manual selection of data time points. iFR was calculated using the method descried by Sen et $a l^{73}$, using dedicated MATLAB software (CardiacWaves, King's College London, London, UK), with data showing good agreement with proprietary software ${ }^{19,117}$. For FFR, the value recorded during an IV adenosine infusion was the lower 5 beat average, as per the 'smart minimum' method ${ }^{47}$. Where IC adenosine was used, the lowest of 3 beat average reading was taken.

Pressure/Doppler data: Peak coronary flow velocity, distal coronary pressure (Pd) and central aortic pressure $(\mathrm{Pa})$ were ECG-gated and recorded continuously at a sampling frequency of $200 \mathrm{~Hz}$ and data exported into a custom-made StudyManager program (Academic Medical Center, University of Amsterdam, Netherlands). The raw data was transferred to optical media and hSR calculated using the dedicated MATLAB software (CardiacWaves, King's College London, London, UK). In order to account for beat-to-beat variability and reduce the effect of noise, signals were ensemble averaged over five consecutive cardiac cycles during stable hyperaemia. Premature ectopic beats and the beat preceding were excluded from analysis. hSR was calculated as $\mathrm{P}_{\mathrm{a}}-\mathrm{P}_{\mathrm{d}} / \mathrm{APV}$, with APV being average peak coronary flow velocity. Significant disease was defined by $\mathrm{hSR} \geq 0.80$.

\section{Statistical Analysis}


All data are expressed as medians [1st quartile; 3rd quartile] or means (standard deviation) for continuous variables (compared using a t-test or ANOVA for continuous normal distributed variables, and Kruskal-Wallis test if continuous non-normal distributed); categorical variables are expressed as absolute and relative frequencies (compared using a Pearson chi-square test). Hypothesis testing was two-tailed and $\mathrm{p}$ values $<0.05$ were considered statistically significant.

Diagnostic accuracy was quantified by the area under receiver-operating curve (AUC $(95 \%$ confidence interval) against $\mathrm{hSR} \geq 0.8$ ). Bootstrapping was used to calculate Confidence Intervals (CI), using 1,000 stratified bootstrap replicates, to compare the AUC between indices and calculate the classification function. Diagnostic accuracy was calculated as $\Sigma$ True positive $+\Sigma$ True negative / $\Sigma$ Total population for each threshold. Correlation was assessed with Pearsons's $\mathrm{R}$ and adjusted $\mathrm{R}^{2}$ by fitting a linear regression model. All statistical analyses were performed using R, version 3.4.3 GUI 1.70 (The R Foundation for Statistical Computing), using packages ggplot2, RMarkdown, pROC and the tidyverse.

\section{Results}

Following exclusion of 6 cases due to poor Doppler flow signals, 75 patients, were included in the study with baseline demographics summarized in Table 1 . Mean age was 62 with $56 \%$ male. One vessel was studied per patient, where FFR was $0.78+/-0.12$ and iFR was $0.85+/-0.10$.

The median (interquartile range) of FFR, $\mathrm{iFR}$ and $\mathrm{Pd} / \mathrm{Pa}$ in our study population was 0.8 (0.72$0.87), 0.89(0.82-0.92)$ and $0.92(0.88-0.94)$ respectively. $80 \%$ of cases included in the analysis were in the diagnostically challenging range between FFR $0.6-0.9$. Scatter plots of the correlation between FFR vs. iFR and PdPa vs. iFR are shown in Figure 1. The Pearson's correlation coefficients were 0.76 (FFR versus $\mathrm{iFR}$ ) and 0.94 (for $\mathrm{iFR}$ versus $\mathrm{Pd} / \mathrm{Pa}$ ). 


\begin{tabular}{|l|c|}
\hline Mean Age (years) [IQR] & $62[53 ; 70]$ \\
\hline Gender, n (\%): Male & $42(56 \%)$ \\
\hline Hypertension, n (\%) & $45(60 \%)$ \\
\hline Hyperlipidemia, n (\%) & $53(71 \%)$ \\
\hline Diabetes mellitus, n (\%) & $23(31 \%)$ \\
\hline Smoking, n (\%) & $20(27 \%)$ \\
\hline Previous PCI, n (\%) & $22(29 \%)$ \\
\hline Interrogated vessel, n (\%): & \\
\hline Left Anterior Descending, LAD & $63(84 \%)$ \\
\hline Circumflex, LCx & $3(4 \%)$ \\
\hline Right Coronary Artery, RCA & $8(11 \%)$ \\
\hline
\end{tabular}

Table: Summary of Patient Demographics 

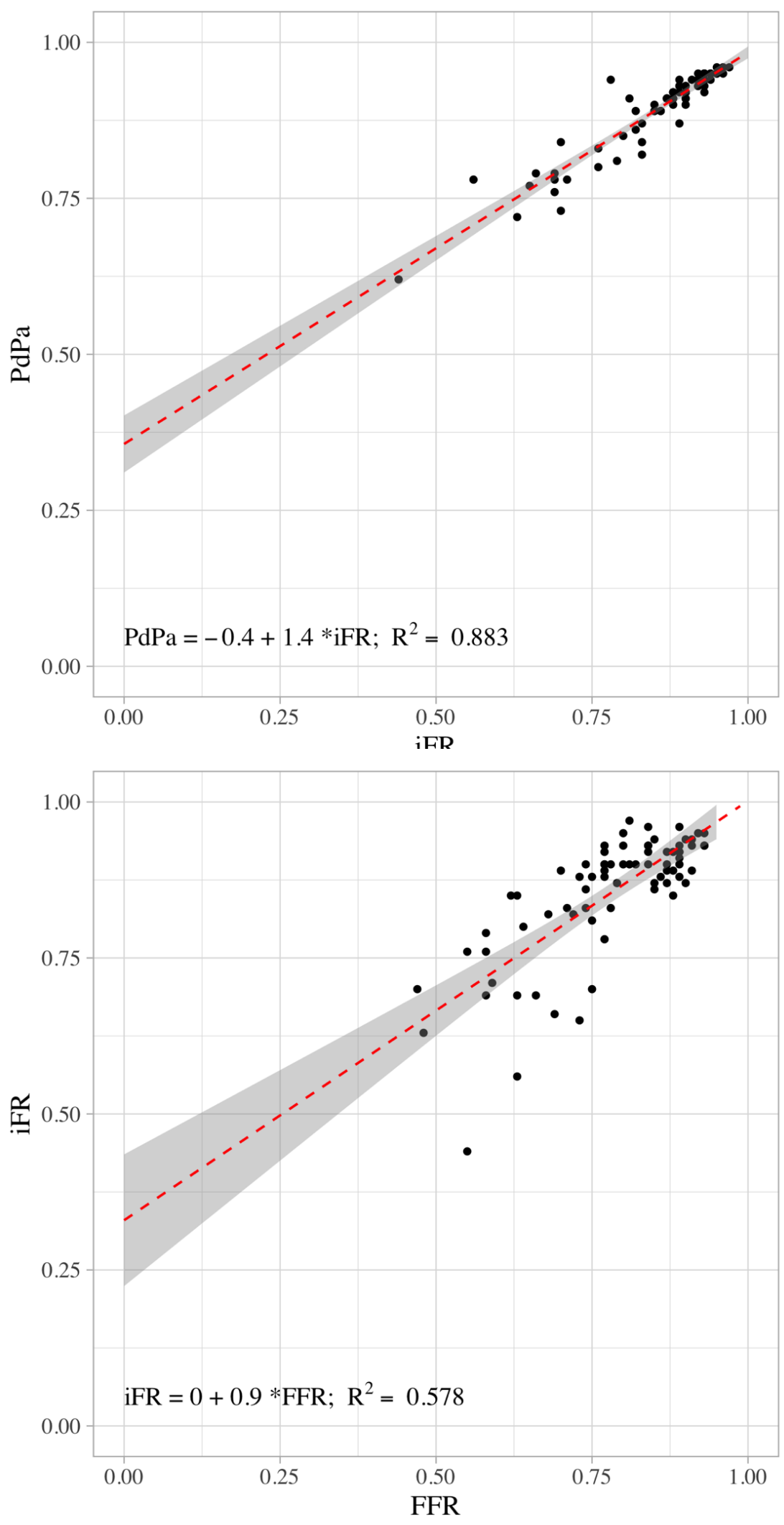

Figure 1: Scatterplots of PdPa versus iFR and FFR versus iFR. Top: Scatterplot of iFR versus $\mathrm{PdPa}$. Grey shaded area represents 95\% confidence interval of trend line. Pearson's R value 0.94, $\mathrm{R}^{2} 0.88(\mathrm{P}<0.05)$. Bottom: Scatterplot of FFR versus iFR. Grey shaded area represents $95 \%$ confidence interval of trend line. Pearson's $\mathrm{R}$ value $0.76, \mathrm{R}^{2} 0.58(\mathrm{P}<0.05)$. 
Receiver Operator Curves (ROC) for the performance of iFR, FFR and $\mathrm{Pd} / \mathrm{Pa}$ in detecting a hSR value $\geq 0.8$ are shown in Figure 2 . FFR had the best diagnostic accuracy ( $p=0.002$ for comparison of AUC of FFR versus $\mathrm{iFR}$ and $\mathrm{Pd} / \mathrm{Pa}$ ). The AUC to predict $\mathrm{hSR} \geq 0.8$ was 0.92 (95\% CI 0.84-0.87), 0.79 (95\% CI 0.66-0.90) and 0.78 (95\% CI $0.64-0.9)$ for FFR, iFR and Resting $\mathrm{Pd} / \mathrm{Pa}$ respectively.

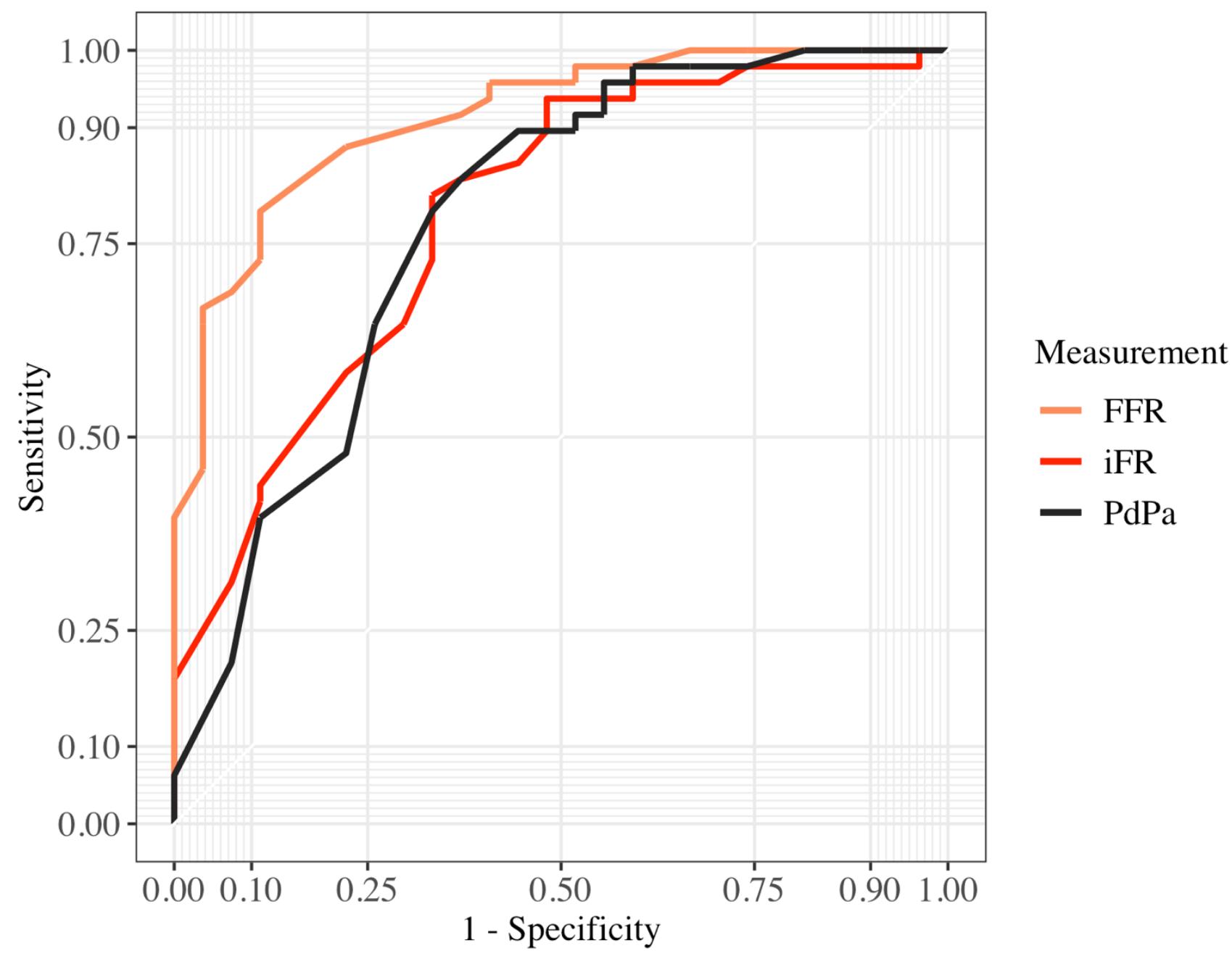

Figure 2: $\mathrm{ROC}$ curves for FFR, $\mathrm{iFR}$ and $\mathrm{PdPa}$ with comparison made against $\mathrm{hSR}>0.8$ to identify ischaemia. AUC for FFR 0.92 (0.85-0.97 95\% CI), iFR 0.79 (0.67-0.89 95\% CI) and $\mathrm{PdPa} 0.77$ (0.65-0.89 95\% CI). FFR AUC was significantly different $(\mathrm{P}=0.003)$ compared to iFR and PdPa AUCs. 

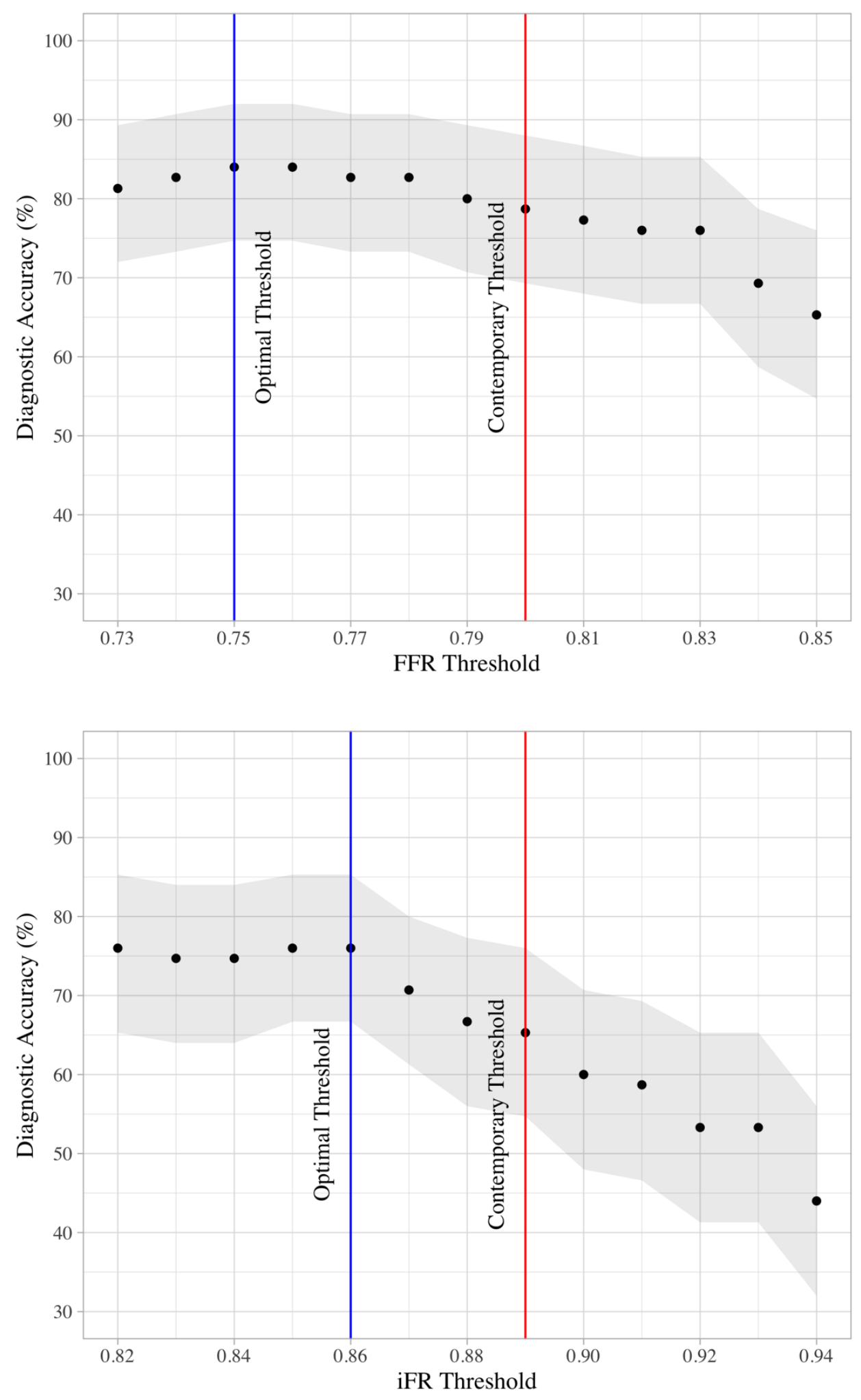

Figure 3: Charts Showing Diagnostic Accuracy of Varying FFR and iFR Thresholds. Graphs comparing diagnostic accuracy of varying thresholds of FFR (Top) and iFR (Bottom). Greyed area represents $95 \%$ confidence interval. Blue Line represents the optimal threshold identified in this study versus an invasive reference standard. Red line identifies the contemporary threshold used for each index in current clinical practice. 
Sensitivity, specificity, NPV, and PPV for the contemporary FFR threshold $(\leq 0.80)$ were $89.3 \%$, $73.5 \%, 92.5 \%$ and $65.7 \%$ respectively, with an overall diagnostic accuracy of $78.7 \%$. Peak diagnostic accuracy was found at FFR thresholds of 0.75 - 0.76 (diagnostic accuracy 84\%). Sensitivity, specificity, NPV, and PPV for the contemporary iFR threshold $(\leq 0.89)$ were $78.3 \%$, $58 \%, 82.9 \%$ and $51.2 \%$ respectively, with an overall diagnostic accuracy of $65.3 \%$. Peak diagnostic accuracy was found at iFR thresholds of $0.85-0.86$ (76\%). Peak diagnostic accuracy for resting $\mathrm{Pd} / \mathrm{Pa}$ was found at a threshold of 0.88 (55.6\% Sensitivity, $89.8 \%$ specificity, $78.7 \%$ NPV, 76\% PPV and an overall diagnostic accuracy of 77.3\%). A graphical representation of the diagnostic accuracy of different thresholds of FFR and iFR is shown in Figure 3.

Using contemporary thresholds, there was discordance between FFR and iFR in $25.3 \%$ of cases with an equal number of cases where FFR +ve / iFR - ve and iFR +ve / FFR - ve. There was no significant difference $(\mathrm{p}>0.05)$ in baseline characteristics or coronary flow reserve (CFR) between these discordant groups. When adopting the optimal iFR and FFR thresholds from this study, the discordance between FFR and iFR fell to 10.7\%.

\section{Discussion}

The main findings of this study are:

1) The optimal diagnostic thresholds for FFR and iFR were found to be 0.75 and 0.86 respectively, which are in keeping with the thresholds identified when each index was originally derived. These thresholds provide superior diagnostic accuracy than contemporary thresholds for FFR $(\leq 0.80)$ and iFR $(\leq 0.89)$.

2) The hyperaemic index FFR provides superior diagnostic accuracy to the resting indices, iFR and $\mathrm{Pd} / \mathrm{Pa}$, whilst the diagnostic accuracies of the latter are comparable.

Our results suggest that contemporary iFR (0.89) and FFR (0.80) thresholds provide suboptimal 
diagnostic performance compared to the original thresholds from derivation studies. With evidence to suggest the benefits of revascularizing stable CAD may not be as significant as originally anticipated and many questioning the role of PCI in stable CAD ${ }^{113}$, the 'safety net' of adopting higher thresholds with higher NPVs in exchange for lower overall diagnostic accuracy may be unjustified, particularly given the low event rate often seen in large trials of stable $\mathrm{CAD}^{3,4,11,12}$.

Adopting more rigorous thresholds means more targeted revascularisation of those patients with a greater burden of ischaemia: with significant evidence existing to suggest greater clinical benefit and the recent ACC/AHA Appropriate Use Criteria recognizing patients with a greater burden of ischaemia are higher risk and more likely to benefit from revascularisation ${ }^{104}$. The Hachamovitch et al cohort study of over 10,000 patients showed revascularisation compared with medical therapy had greater survival benefit (absolute and relative) in patients with moderate to large amounts of inducible ischaemia ${ }^{7}$ and this evidence was supported by the nuclear sub-study of COURAGE, demonstrating that patients with a $\geq 5 \%$ reduction of ischaemia on MPS had better outcomes from revascularisation compared to medical therapy ${ }^{118}$. In addition, in recent years, meta-analysis of FFR data has suggested lesions with lower FFR values receive larger absolute benefits from revascularisation ${ }^{119}$, with the analysis suggesting that an FFR as low as 0.67 provides optimal benefit for a composite of death, MI, and revascularisation. The definitive evidence that revascularizing patients with greater ischaemic burden yields greater patient benefit will come in the form of the ongoing ISCHAEMIA trial (NCT01471522). Until this trial reports, there exists substantial observational data to suggest patients with the greatest ischaemic burden are likely to experience most benefit from revascularisation and therefore the more rigorous iFR and FFR thresholds that this study supports, may well translate into improved clinical outcomes.

Whilst our study is not the first to demonstrate significant discordance between FFR and iFR, it is the first to do so for modern thresholds using an invasive reference standard for ischaemia resulting from epicardial CAD. Very few studies have correlated iFR and FFR versus an 
independent reference standard. Petraco et al used coronary flow reserve (CFR) as an independent reference to show that an iFR threshold of 0.9 had better diagnostic discrimination than an FFR of 0.8 (iFR AUC 0.82; FFR AUC 0.72; $<<0.001)^{120}$. Using CFR as a gold standard for invasive physiological measures of epicardial coronary disease may be significantly confounded by variability in microvascular function. Hwang et al have used PET Relative Flow Reserve to show no significant difference in AUC between iFR and FFR (iFR AUC 0.77; FFR AUC $0.83 ; \mathrm{P}=0.05$ ) but with FFR showing better discriminatory ability when compared to an iFR threshold of $0.9^{121}$, in keeping with the findings of our study. Sen et al previously also used hSR, to show similar diagnostic performance of iFR and FFR in 51 patients (iFR AUC 0.93; FFR AUC 0.96; $\mathrm{P}=0.48$ ), although this study included patients with a milder spectrum of disease; less than $2 / 3^{\text {rd }}$ were within the diagnostically challenging range $0.60-0.90^{87}$. In comparison to these studies we present the largest cohort $(n=75)$ in which an invasive reference standard for ischaemia has been applied ${ }^{21,42}$ with $80 \%$ of patients in our study within the diagnostically uncertain FFR range between 0.6 and 0.9 .

The implications of this work are wide-ranging, including the suggestion that clinicians should consider the original and more stringent thresholds in future studies of both FFR and iFR. This is further supported by the recent data by Kang et al suggesting PCI of greyzone-FFR cases do not derive net clinical benefit ${ }^{116}$. If future trials for physiology-guided revascularisation did use more stringent thresholds, this would mean revascularisation is reserved for a higher risk cohort which may yield greater benefit than the surprisingly modest outcomes and weak symptom benefit suggested by some recent trials of PCI for stable CAD ${ }^{112,113}$.

\section{Study Limitations}

We recognize there is currently no gold standard ischaemia test, and this is reflected by the fact previous studies have used PET, CFR and hSR as a reference standard, without consensus. We used hSR, as this the most theoretically robust invasive vessel-specific physiological test, which is known to have greater accuracy in detecting inducible ischaemia ${ }^{21}$ and has previously been used 
in derivation work of pressure-derived indices of stenosis severity ${ }^{87}$. However, hSR lack the prognostic evidence base that the pressure derived indices have and given the steep learning curve, its use is limited to the research arena.

We also recognize that this study has a relatively small sample size from a single centre. Whilst it is certainly the largest study of its kind, lend support to a large multi-centre randomized study, rather than being practice changing per se.

\section{Conclusion}

Modern FFR and iFR thresholds provide suboptimal diagnostic accuracy compared to a FFR threshold of 0.75 or an iFR threshold of 0.86 respectively, which are also the binary cut-offs from the original derivation studies. Fractional flow reserve had better diagnostic accuracy than either of the resting indices, $\mathrm{iFR}$ and $\mathrm{Pd} / \mathrm{Pa}$. When used to guide treatment, the less specific contemporary thresholds could lead to inappropriate treatment of vessels. Whether the use of the more rigorous thresholds would result in selection of a population gaining greater symptom and prognostic benefit needs to be assessed in future studies of physiology-guided revascularisation. 


\title{
A2: IS HEART RATE RESPONSE A RELIABLE MARKER OF ADENOSINE-INDUCED CORONARY HYPERAEMIA?
}

\begin{abstract}
Purpose: Growing evidence supports ischaemia-guided management of chest pain, with invasive and non-invasive tests reliant upon achieving adenosine-induced coronary hyperaemia (defined as increased blood flow to an organ's perfusion bed). In the non-invasive setting, surrogate markers of hyperaemia, such as increases in heart rate, are often used, despite not being formally validated. We tested whether heart rate and other non-invasive indices are reliable markers of coronary hyperaemia. Methods: The first part involved Doppler flow-based validation of the best pressure-wire markers of hyperaemia in 53 patients. Subsequently, using these validated pressurederived parameters, 265 pressure-wire traces were analysed to determine whether heart rate and other non-invasive parameters correlated with hyperaemia. Results: In the flow derivation cohort, the best determinant of hyperaemia came from having 2 out of 3 of: 1) Ventriculisation of the distal pressure waveform, 2) Disappearance of distal dicrotic pressure notch, 3) Separation of mean aortic and distal pressures. Within the 244 patients demonstrating hyperaemia, non-invasive markers of hyperaemia, such as change in heart rate $(p=0.77)$, blood pressure $(p=0.60)$ and ratepressure product $(\mathrm{p}=0.86)$, were poor correlates of coronary hyperaemia, with only $37.3 \%$ demonstrating $\mathrm{a} \geq 10 \%$ increase in heart rate that is commonly used to adjudge adenosine-induced hyperaemia in the non-invasive setting. Conclusions: We demonstrate, by correlation with Doppler-flow data, a validated method of identifying coronary hyperaemia within the catheter laboratory using the pressure-wire. We subsequently show that non-invasive parameters, such as heart rate change, are poor predictors of coronary hyperaemia during stress imaging protocols that rely upon achieving adenosine-induced hyperaemia.
\end{abstract}

\section{Introduction:}

A growing body of evidence supports ischaemia-guided revascularisation ${ }^{7}$. Surrogates of ischaemia can be assessed non-invasively or during diagnostic angiography, often relying on 
pharmacological induction of coronary hyperaemia ${ }^{122}$. The most widely used invasive measure is Fractional Flow Reserve (FFR) $)^{2,4,10}$, based on the measurement of distal coronary and aortic pressure during adenosine-induced hyperaemia. Intravenous adenosine is also used in stress perfusion Cardiac Magnetic Resonance Imaging (CMR). This test is increasingly used to detect and quantify ischaemia in patients with suspected coronary disease ${ }^{123}$ by demonstrating regional heterogeneity of coronary blood flow during hyperaemia. Intravenous (IV) adenosine, at a dose of $140 \mathrm{mcg} / \mathrm{kg} / \mathrm{min}$, has been shown to reliably induce near-maximal hyperaemia in most patients, with minimal side-effects ${ }^{124}$. The net effect of IV adenosine in humans is typically a mild reduction in arterial blood pressure associated with increases in heart rate (HR), with multiple mechanisms proposed ${ }^{124,125}$. Due to its non-selectivity, adenosine also activates other receptors $\left(\mathrm{A}_{1}, \mathrm{~A}_{2 \mathrm{~B}}\right.$ and $\left.\mathrm{A}_{3}\right)$, which can also result in cardiac conduction abnormalities, hypotension, flushing and bronchospasm ${ }^{126}$.

True hyperaemia is best assessed by showing increases in coronary blood flow measured invasively using Doppler or Thermodilution techniques, that are difficult to implement outside the research setting. Predicting when a patient is experiencing maximal hyperaemia within the catheter laboratory is therefore sometimes assessed by awaiting the onset of flushing, breathlessness and chest tightness symptoms. Additionally, non-invasive surrogates such as blood pressure drop, HR rise and changes in aortic and distal coronary pressure waveforms are relied upon to determine the onset of hyperaemia, although no reproducible and objective criteria have been identified.

Within the non-invasive CMR setting, where it is not possible to measure such invasive indices, subjective symptoms along with objective hemodynamic measures of increasing HR and falling systolic blood pressure (SBP) are used as surrogate markers of hyperaemia. $10 \%$ or 10 beats per minute increase in $\mathrm{HR}$, is commonly considered a marker of adequate hyperaemia within the imaging setting, its absence thought to imply inadequate hyperaemic stimulus. In these cases, higher adenosine doses are administered or the study is classified as equivocal ${ }^{127}$. A sub-analysis 
of the CE-MARC study suggests that inadequate hyperaemic response is considered a recognized cause of a false-negative CMR Perfusion $\operatorname{scan}^{128}$.

In this study, we tested the hypothesis that HR changes, and other surrogate non-invasive indices are reliable markers of coronary hyperaemia.

\section{Methods}

\section{Study population}

Our study population consisted of patients who presented to a single centre for coronary angiography $+/$ - proceeding to percutaneous intervention as appropriate. 306 consecutive patients undergoing FFR measurements between October 2013 and February 2017 were screened, where hyperaemia was induced by IV adenosine infusion. Between this period, 53 patients also had simultaneous pressure and Doppler measurements using a CombowireXT guidewire (Philips Volcano) as part of a number of studies utilizing detailed intracoronary physiological measurements in patients with ischaemic heart disease. All patients received an IV adenosine infusion dose of $140 \mathrm{mcg} / \mathrm{kg} / \mathrm{min}$ through an antecubital vein using a standardized infusion pump at a fixed distance from the patient, to minimize variability. For the purposes analysis, FFR was defined as the lowest $\mathrm{Pd} / \mathrm{Pa}$ ratio following the onset of adenosine, averaged over 5 cardiac cycles, also known as the 'smart minimum FFR'47. All participants gave written informed consent in accordance with the protocol approved by the local research ethics committee. The study protocol conformed to the ethical guidelines of the 1975 Declaration of Helsinki.

\section{Standardisation of Invasive Pressure Assessment of Hyperaemia}

Hyperaemia was assessed in the 53 patients using Doppler flow velocity measurements, by examining Coronary Flow Reserve (CFR), defined as the ratio of Average Peak Flow Velocity (APV) compared to baseline. Doppler measurements of coronary blood flow velocity have been shown to have inter- and intra-observer variability of approximately $10 \%{ }^{129}$. A pre-defined CFR threshold of 1.2 was therefore used to define hyperaemia (defined as an increase in blood flow to 
an organ's perfusion bed) to ensure that the increase in flow at hyperaemia is above the margin of measurement error commonly seen with CombowireXT Doppler flow measurements.

In this Doppler cohort, we assessed the diagnostic performance of three commonly-used invasive pressure-waveform parameters of hyperaemia, and combinations thereof by calculating their sensitivity, specificity and positive and negative predictive values. These are 1) Ventricularisation of distal pressure waveform (a presystolic deflection resembling an 'a wave', a slower upstroke of the waveform and a steeper down-stroke than that of aortic pressure ${ }^{130}$ ), 2)Separation of mean aortic and distal coronary pressure $(>10 \%$ difference in $(\mathrm{Pa}-\mathrm{Pd})$, over 5 consecutive heart beats, compared to the resting gradient) and 3)Disappearance of dicrotic notch from the distal arterial pressure trace (see Figure 1). In the absence of an established CFR cut-off for defining hyperaemia, the diagnostic performance of the pressure-based parameters at CFR of 1.2 were also compared to their performance at a higher CFR of 1.5.

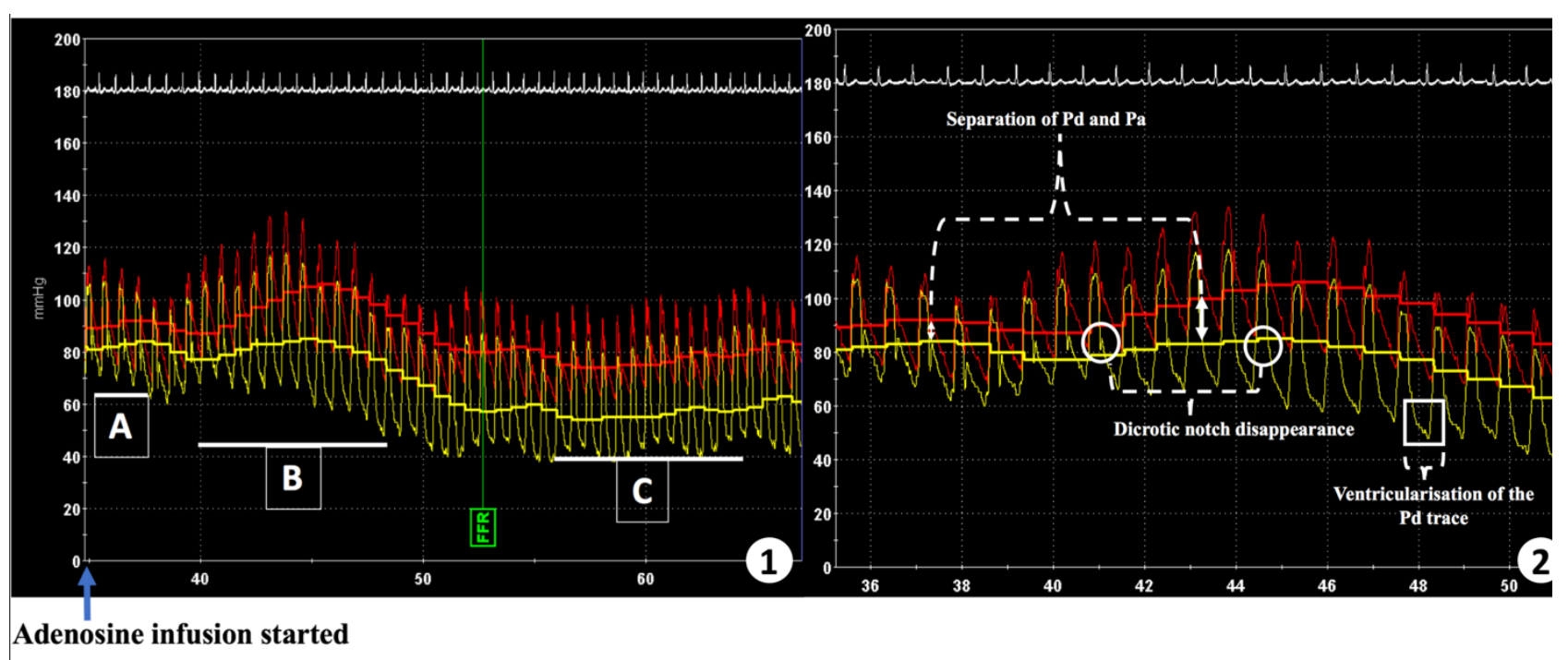

Figure 1 Pressure-bounded invasive parameters

a. The 3 invasive pressure-based parameters that were investigated and subsequently used to define hyperaemia during IV Adenosine infusion. Red trace $=\mathrm{Pa}$ (Aortic Wave) Yellow trace $=\mathrm{Pd}($ Distal Coronary Wave $)$ b. Magnification of the 3 invasive pressure-bounded parameters 


\section{Assessing Adenosine-Mediated Changes in Coronary Microvascular Resistance and Peripheral Vascular Resistance}

The flow cohort enabled characterization of coronary microvasculature resistance (MR) and peripheral vascular resistance (via the augmentation index, AIx) ${ }^{131}$, calculated over five consecutive beats at rest and hyperaemia. MR was defined as the distal pressure divided by APV. AIx; a measure of central aortic pressure-waveform enhancement by a reflected pulse wave, is calculated as the difference between this late systolic pressure $P_{2}$ and early systolic pressure $P_{1}$, as a percentage of pulse pressure, whereby $P_{l}$ was identified as the first peak on an aortic pressure wave (resulting from the ejection of blood from the heart) and $P_{2}$ identified as the second peak (resulting from reflection of blood due to constriction downstream in the peripheral vascular tree).

\section{Assessing Diagnostic Performance of Heart Rate and Other Non-Invasive Surrogate Markers of Hyperaemia}

Based on the diagnostic performance of the invasive pressure parameters (and combinations thereof) in the Doppler cohort, the study population $(n=265)$ were dichotomously classified as hyperaemic or non-hyperaemic. The predictive accuracy of commonly used non-invasive haemodynamic markers (HR, SBP, and rate pressure product [RPP, HRxSBP]) were analysed as a percentage change in each parameter in response to IV adenosine-induced hyperaemia.

\section{Statistical Analysis}

Continuous variables were assessed for normality and if found to be normally distributed, were expressed as mean \pm standard deviation. Categorical variables were expressed as counts and percentages. Differences in continuous variables were assessed by an independent Student's t-test, whilst differences in categorical variables were evaluated by Fisher's exact Chi-Squared test. Differences in continuous matched variables, such as HR at rest and at hyperaemia were assessed using a Paired T-test. The diagnostic accuracy of invasive pressure-waveform parameters at detecting a CFR $>1.2$ was classified in terms of specificity, sensitivity, positive predictive value (PPV) and negative predictive value (NPV). Changes in microvascular resistance and peripheral 
vascular resistance were correlated using Pearson's Rank Correlation, after testing for normality, and results reported a $\mathrm{R}^{2}$ values. Statistical analyses were performed using SPSS software (version 22.0; SPSS Inc., Chicago, Ill., USA).

\section{Results}

\section{Performance of Invasive Pressure-Indices of Hyperaemia}

In the 53 patients with simultaneous pressure and Doppler measurements, mean FFR was $0.84 \pm 0.1$. The individual pressure-waveform indices all had good sensitivity at detecting hyperaemia, defined using a CFR threshold of 1.2, but relatively low specificity. Disappearance of the dicrotic notch and ventricularisation of the distal pressure waveform were the two parameters achieving the best NPV and PPV (Figure 2 and Table I). The reduced diagnostic performance of $\mathrm{Pd}$ and $\mathrm{Pa}$ trace separation (Figure 2), can be explained by the fact that this parameter is difficult to detect in normal coronary arteries (FFR $>0.9)$. In contrast dicrotic notch disappearance and ventricularisation were detected easily, regardless of disease burden. Combining these indices improved specificity and hence the presence of at least $2 / 3$ pressure indices was chosen as the optimum criterion for detecting hyperaemia, forming the basis of detecting hyperaemia in the pressure cohort. 


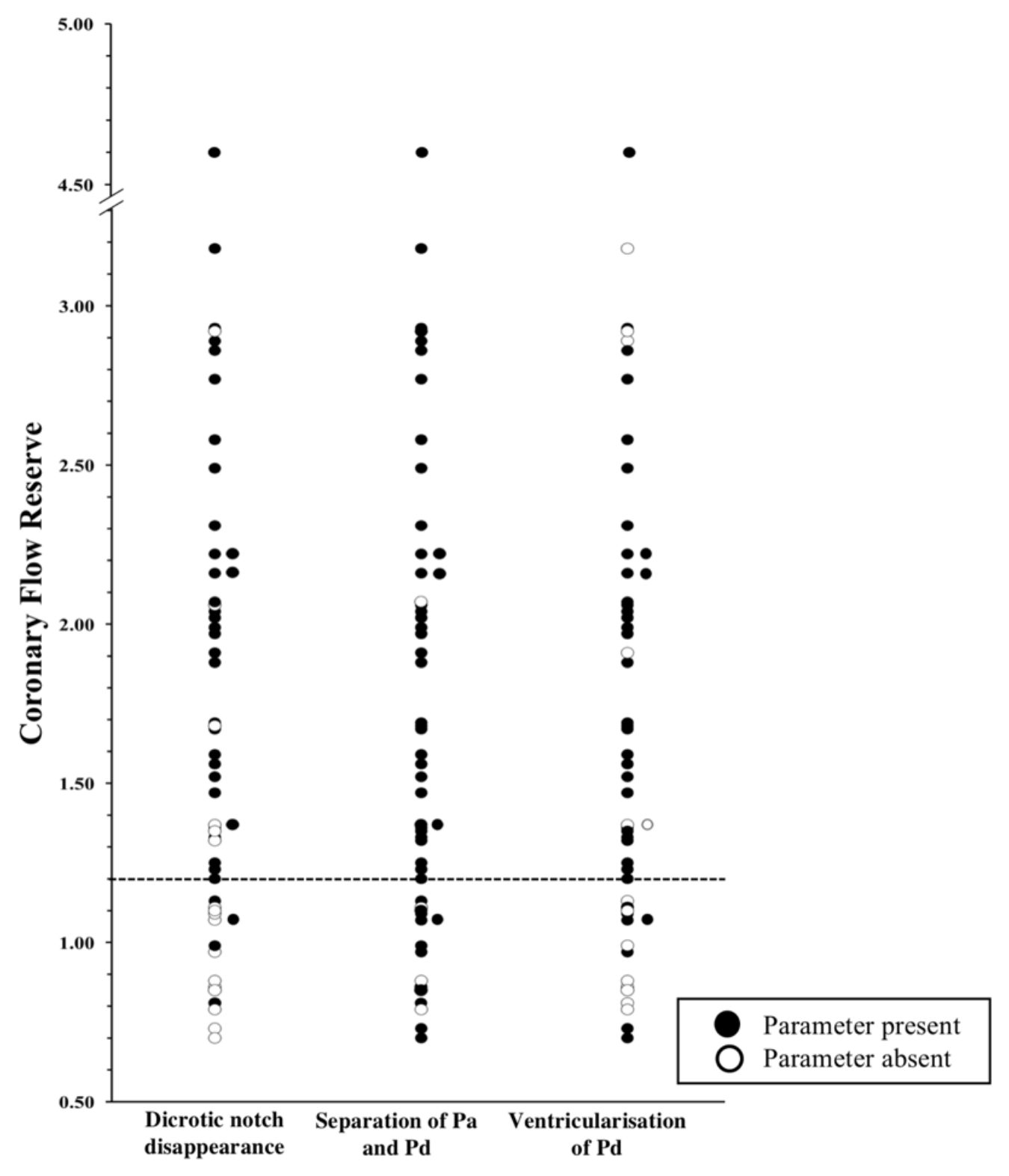

Three types of parameters on a pressure trace

Figure 2 Relationship between Coronary Flow Reserve (CFR) and the 3 invasive parameters of hyperaemia: Graph illustrating the presence and absence of pressure-based parameters of hyperaemia in 53 patients where simultaneous CFR value were measured. CFR cut-off of 1.2 used as a marker of definitive hyperaemia

When a higher CFR threshold of 1.5 was used, the diagnostic performance of the pressurebounded parameters was almost identical to a CFR of 1.2. However, by using a CFR threshold of 1.5, hyperaemic and non-hyperaemic rates were found to be clinically unrealistic at $53 \%$ and 47 $\%$ respectively. 


\begin{tabular}{lcccc}
\hline & Sens & Spec & NPV & PPV \\
\hline Dicrotic notch disappearance & 84.2 & 73.3 & 88.9 & 64.7 \\
\hline Separation of Pa and Pd & 97.4 & 20 & 75.5 & 75 \\
\hline Ventricularisation of Pd trace & 84.2 & 53.3 & 82.1 & 57.1 \\
\hline Dicrotic notch disappearance & 73.7 & 93.3 & 96.6 & 58.3 \\
+ Ventricularisation & & & & \\
\hline Dicrotic notch disappearance & & & & \\
\hline + Separation of Pa and Pd & 81.6 & 73.3 & 88.6 & 61.1 \\
\hline Separation of Pa and Pd & & & & \\
\hline \\
+ Ventricularisation
\end{tabular}

Table I Diagnostic performance of each invasive parameter, and combinations of 2, at a CFR threshold of 1.2 Within the validation cohort of patients with pressure and flow data, a CFR cut-off of 1.2 was used to calculate sensitivity (Sens), specificity (Spec), negative predictive (NPV) and positive predictive value (PPV) of the three pressure based parameters. Diagnostic performance was assessed both on their own and/or in different paired combinations 


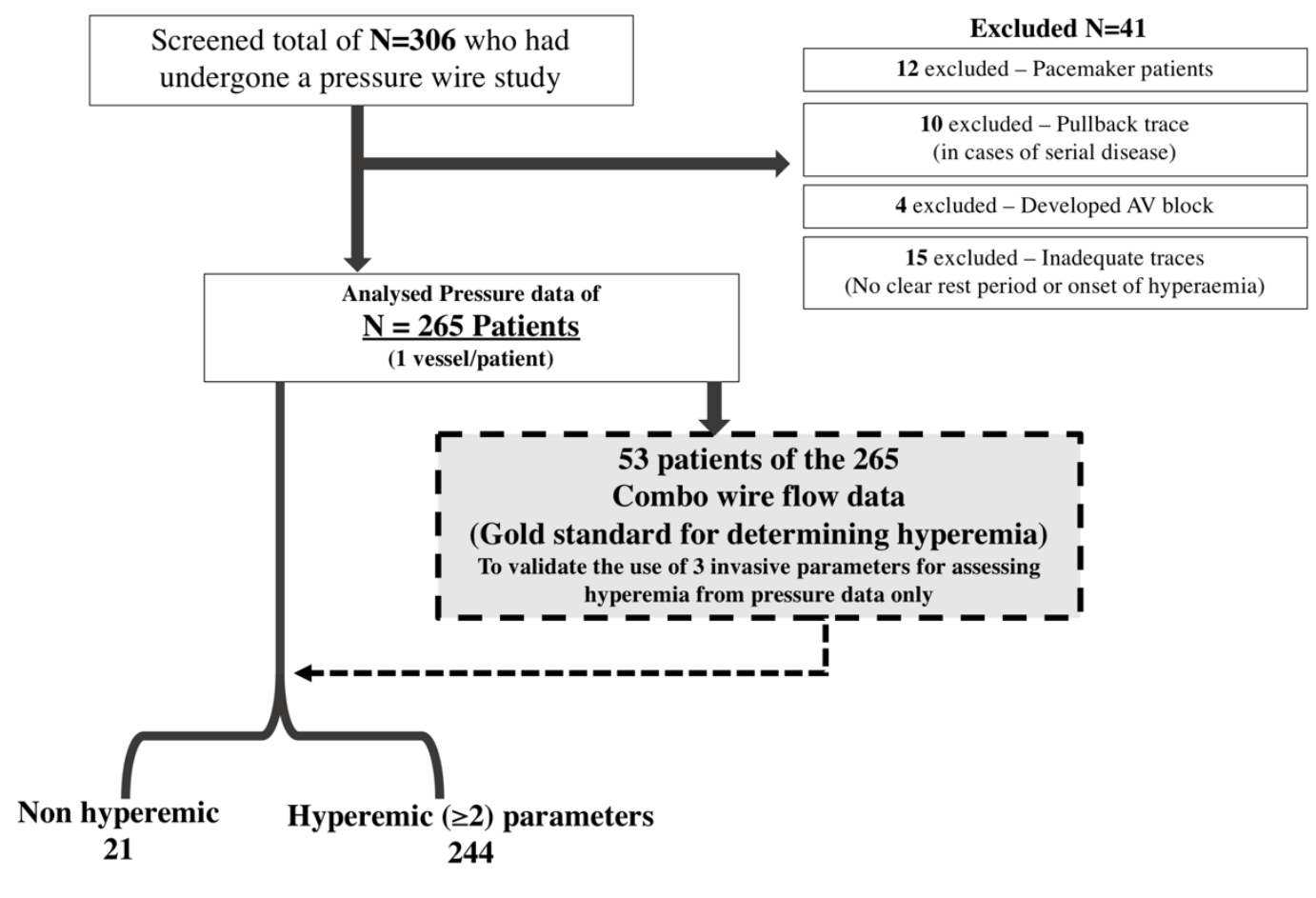

Figure 3 Flow chart: 265 patients with pressure data were analysed using the flow-validated pressure indices to determine hyperaemic or not. The predictive accuracy of commonly used non-invasive haemodynamic markers (heart rate [HR], systolic blood pressure [SBP] and rate pressure product [HR x SBP]) were analysed as \% change in each parameter in response to IV adenosine from rest to the onset of the lowest $\mathrm{Pd} / \mathrm{Pa}$ ratio

\section{Changes in Coronary Microvascular Resistance and Peripheral Vascular Resistance}

Using flow data from 53 patients, we found both the MR and AIx significantly dropped from rest to hyperaemia $(-37 \pm 29.76 \% \mathrm{p}<0.001$ and $-7.13 \pm 55.32 \%, \mathrm{p}=0.004)$. There was no correlation between HR change and AIx, $\mathrm{R}^{2}=0.031, \mathrm{p}=0.2$ (2-sided), or between changes in MR and AIx from rest, $\mathrm{R}^{2}=0.021, \mathrm{p}=0.3$.

\section{Dichotomization of patients to hyperaemic and non-hyperaemic:}

306 consecutive patients undergoing invasive FFR measurements between October 2013 and February 2017 were screened, where hyperaemia was induced by IV infusion of adenosine. 265 patients were analysed; 41 patients were excluded for reasons specified in the study flow chart 
(Figure 3). Based on our validated pressure-based criteria, 244 of the 265 patients were determined to have developed hyperaemia.

\section{Patient Characteristics}

The enrolled population of 265 patients was $65+/-11$ years old with $74 \%$ male. We assessed one vessel per patient and found the mean FFR, in cases where hyperaemia was adjudged to have been reached, was $0.81+/-0.09$. Although the proportion of patients with previous PCI was higher patients determined to have reached hyperaemia compared to those that did not, there were no other significant differences in patient characteristics between the hyperaemic and nonhyperaemic groups (Table II). 


\begin{tabular}{|c|c|c|c|c|c|}
\hline \multirow[b]{2}{*}{ Variables } & \multicolumn{2}{|c|}{ Hyperaemic } & \multicolumn{2}{|c|}{ Non-hyperaemic } & \multirow[t]{2}{*}{$P$ value } \\
\hline & $N=244$ & $\%$ & $\mathbf{N}=\mathbf{2 1}$ & $\%$ & \\
\hline Age & $65 \pm 10.8$ & & $\begin{array}{c}67 \pm \\
9.7\end{array}$ & & 0.63 \\
\hline $\operatorname{Sex}(M / F)$ & $181 / 63$ & $74.2 / 25.8$ & $16 / 5$ & $76.2 / 23.8$ & 0.54 \\
\hline Hypertension & 152 & 62.3 & 10 & 47.6 & 0.14 \\
\hline Hypercholesterolemia & 181 & 74.2 & 16 & 76.2 & 0.54 \\
\hline Diabetes Mellitus & 59 & 24.2 & 8 & 38.1 & 0.13 \\
\hline Smoker & 49 & 20.1 & 4 & 19 & 0.59 \\
\hline Patients with a history of MI & 61 & 25 & 3 & 14.3 & 0.21 \\
\hline Patients with a history of PCI & 90 & 36.9 & 3 & 14.3 & 0.03 \\
\hline Patients with a history of CABG & 10 & 4.1 & $\mathbf{0}$ & $\mathbf{0}$ & \\
\hline Indication for PCI: Stable & 221 & 90.6 & 20 & 95.2 & 0.41 \\
\hline Elective & 23 & 9.4 & 1 & 4.8 & 0.41 \\
\hline Indication for PCI: ACS & & & & & \\
\hline
\end{tabular}

Table II Demographics of patient population. Comparison of demographics in hyperaemic and non-hyperaemic groups. Abbreviations: M, male; F, female; MI, myocardial infarction; PCI, percutaneous coronary intervention; CABG, coronary artery bypass graft; ACS, acute coronary syndrome. ${ }^{*} \mathrm{P}$ value calculated by Independent Samples T Test for age variable and Chi-squared significance for remaining variables 


\section{Assessment of Heart Rate and Other Non-Invasive Surrogate Markers of Hyperaemia}

The percentage change in HR from rest did not differ significantly between hyperaemic and nonhyperaemic groups; $7.9 \pm 14.0$ and $7.0 \pm 16.3$ respectively $(\mathrm{P}=0.78)$. In addition, there was no significant difference in the proportion of patients exhibiting a $\geq 10 \%$ increase in HR between these groups; $37 \%$ vs. $34 \%$, p=0.10 (Figure 4). Similarly, when these non-invasive parameters were assessed in the 53 flow-data cohort, there was no significant difference in HR, RPP and SBP from rest between hyperaemic and non-hyperaemic patients, as defined by flow. Of the 38 hyperaemic individuals (as defined by CFR $\geq 1.2$ ), only $47 \%$ showed a $\geq 10 \%$ increase in $\mathrm{HR}$. Overall, assessment of the diagnostic performance of $\mathrm{HR}$ in the validation cohort revealed a sensitivity of $37.3 \%$, specificity of $81 \%$, PPV of $96 \%$ and NPV of $10 \%$. Similarly, when assessing HR in the flow-cohort, where hyperaemia was determined using direct Doppler measurements, HR showed a sensitivity of $47.4 \%$, specificity of $86.7 \%$, PPV of $90 \%$ and NPV of $39.4 \%$. In patients where hyperaemia was achieved, the lowest $\mathrm{Pd} / \mathrm{Pa}$ value occurred at $87.8 \pm 32.6$ seconds: significantly earlier than the mean time to peak HR (Figure 5). Other commonly used non-invasive surrogate markers, such as SBP and RPP, also did not vary significantly between hyperaemic and non-hyperaemic patients (Figure 6).
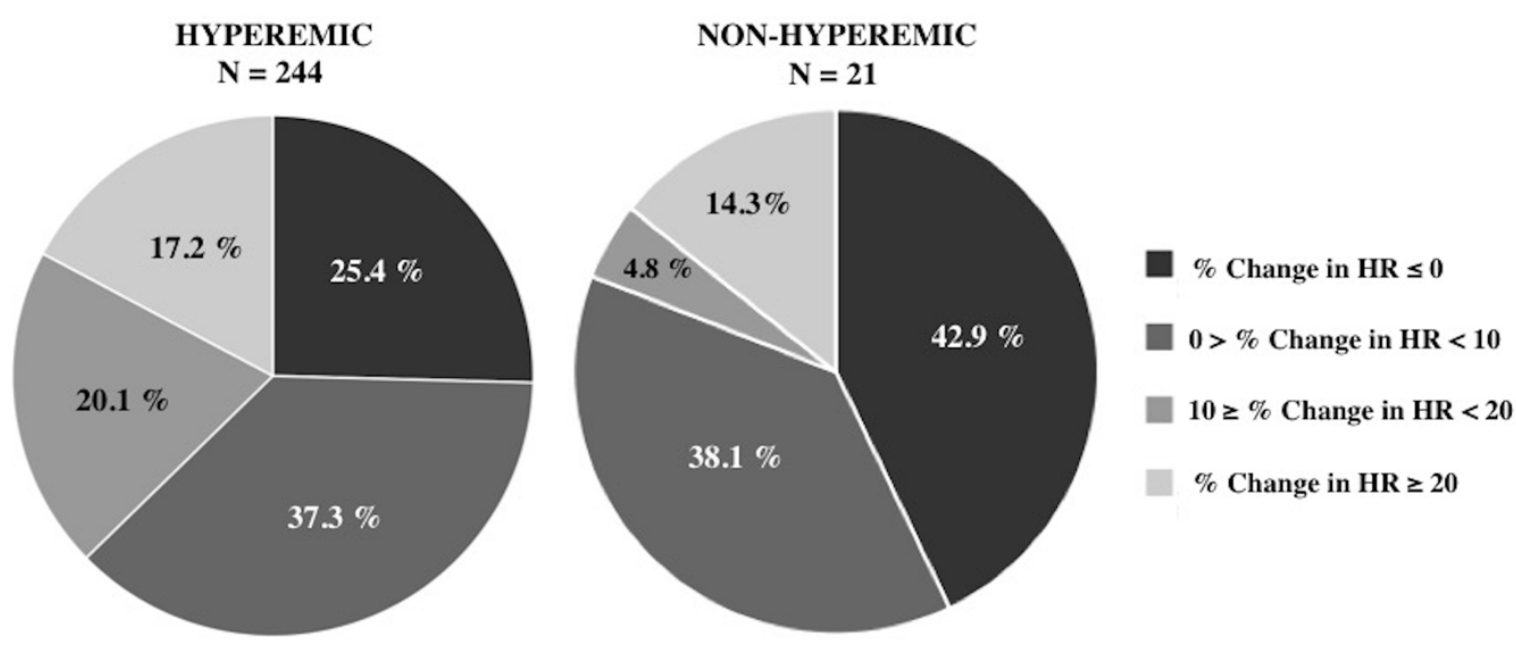

Figure 4 Heart rate variability. Pie chart representation of variability in percentage change in HR in patients deemed to be hyperaemic versus those that were not. 


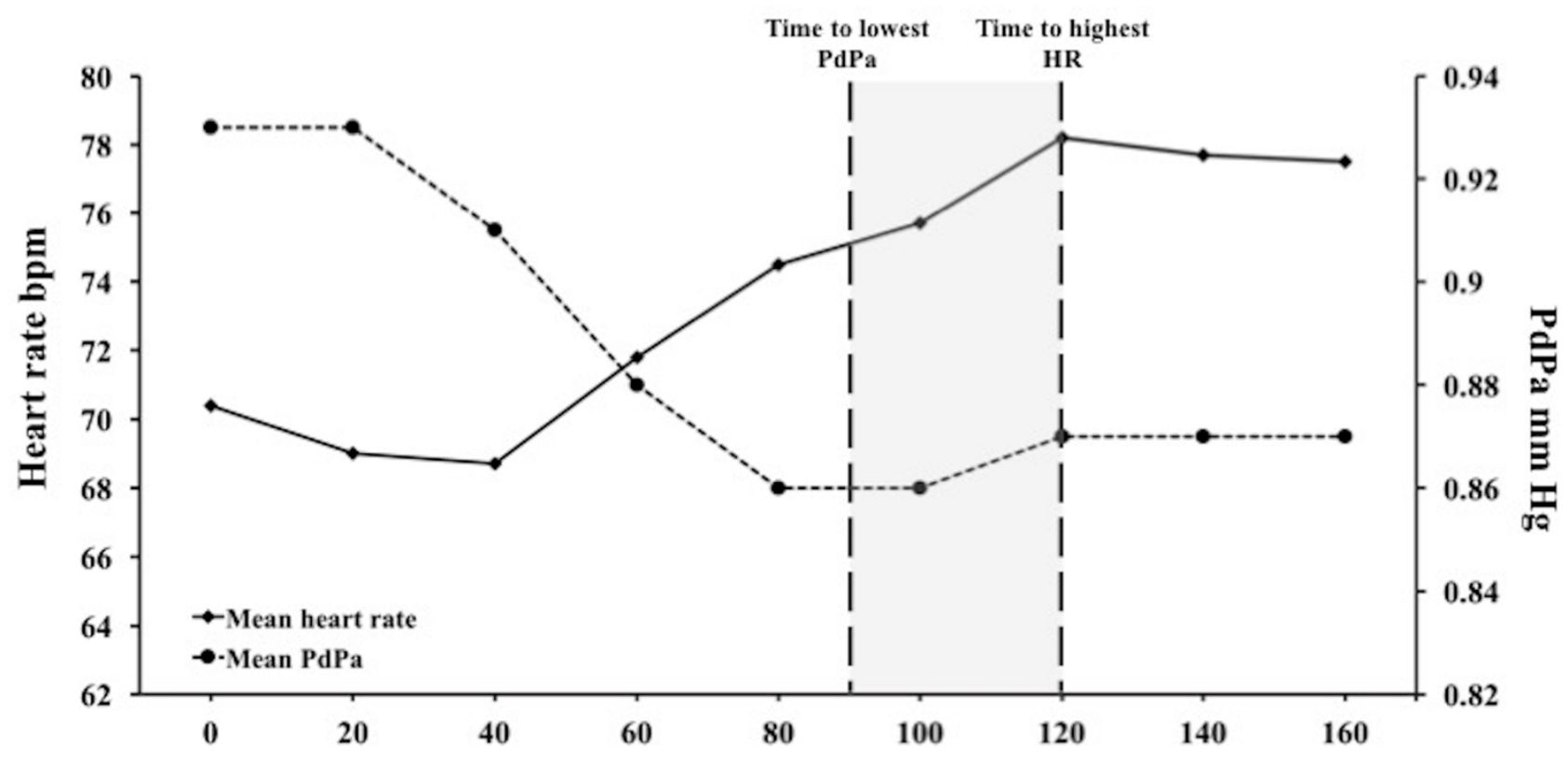

Time from start of IV adenosine infusion (sec)

Figure 5 Variability in Heart Rate and Pd/Pa over time. An illustration of how the mean HR and PdPa changed over the course of adenosine infusion in 244 hyperaemic patients
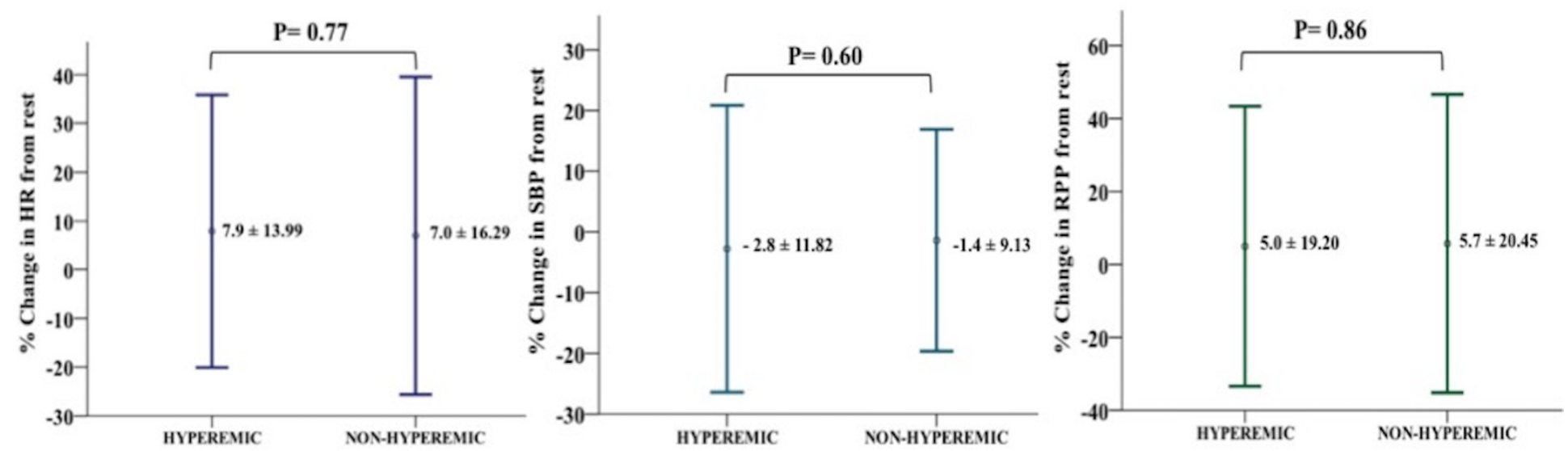

Figure 6 Variability of non-invasive surrogate markers of hyperaemia: A comparison of haemodynamic markers (RPP, HR and SBP) between patients achieving hyperaemia and those that did not. Values are quoted as means \pm standard deviation. 


\section{Discussion}

In this study, we have demonstrated that the tachycardia associated with IV adenosine infusion is an unreliable surrogate marker of maximal hyperaemia, with only $37.3 \%$ hyperaemic patients exhibiting a $10 \%$ increase in HR. This has important implications during non-invasive testing, such as perfusion CMR, when a $10 \%$ (or $10 \mathrm{bpm}$ ) increase in mean $\mathrm{HR}$ is often used as a marker of hyperaemia, although there is no consensus method recommended in the guidelines ${ }^{132,133}$.

The first part of this study demonstrated the best performance for assessing hyperaemia came from having 2/3 of: 1) Ventriculisation of distal pressure waveform, 2) disappearance of distal dicrotic pressure-waveform notch, 3) separation of aortic and distal mean pressures (see Figure 2 and Table I). Using $\geq 2 / 3$ of these parameters, we found $8 \%$ of patients did not exhibit a hyperaemic response. Other studies measuring coronary flow during IV adenosine administration found similar rates of submaximal blood flow ${ }^{124,134,135}$, however studies that adjudged hyperaemia by only demonstrating separation between $\mathrm{Pd}$ and $\mathrm{Pa}$ traces found higher rates of "non-hyperaemic response to IV adenosine' ${ }^{135}$. We demonstrated that this parameter by itself poorly discriminates true hyperaemia (Figure 2), unsurprising in cases of minimal stenoses, where appreciable drops in $\mathrm{Pd} / \mathrm{Pa}$, even during hyperaemia, may not $\operatorname{occur}^{72}$. A CFR of 1.5 was also assessed and whilst similar diagnostic performance was found, the resulting rate of hyperaemia was only $52.8 \%$ which does not reflect a realistic response to adenosine in the clinical setting, nor does it coincide with the rates of hyperaemia reported in previously. Whilst it may be surprising that in the derivation cohort of 53 patients, around half had CFR $<1.5$, this group of patients were pre-selected to consist of an intermediate coronary artery disease severity, whereby associated microvascular dysfunction may have contributed to overall reduced flow augmentation following adenosine administration. A CFR cut-off of 1.2 was therefore used to not only compensate for the classical $10-15 \%$ error of Doppler measurements, but also to yield clinically realistic PPV, NPV and hyperaemia rates, compatible with previous reports ${ }^{124}$. 
Nearly two thirds of patients with demonstrable hyperaemia did not have an accompanying $\geq 10 \%$ HR increase commonly used to infer hyperaemia non-invasively. Consequently, the NPV of HR was found to be poor. The poor performance of HR in the flow-defined group further supports its unreliability as a surrogate marker of hyperaemia. Had this arbiter been applied in the perfusion MRI setting, these patients would have been misclassified as "non-responders", potentially leading to unnecessarily higher doses of adenosine (with more unpleasant side-effects) without added diagnostic value or misclassifying negative perfusion scans as equivocal ${ }^{124,132,136}$. We have also shown that the time-course of HR change may not reflect the onset of maximal hyperaemia (Figure 5), with prolonged adenosine infusions subjecting patients to unpleasant symptoms unnecessarily. Interestingly, we found $12.3 \%$ of patients showed a phasic response to IV adenosine; whilst lower than the $39 \%$ previously quoted ${ }^{47}$, if first pass perfusion image acquisition occurs during the inter-hyperaemic window, the diagnostic value of the scan may be compromised, leading to further diagnostic inaccuracy when using IV adenosine in the noninvasive setting.

\section{Potential mechanisms underlying variation heart rate response to adenosine}

The ability of adenosine to induce tachycardia is ascribed to peripheral vasodilatation, with the assumption that HR change is a direct reflection of peripheral vasodilatation. In addition, both direct and reflex baroreceptor-mediated sympathetic activation are thought to play a role ${ }^{137,138}$. Our study has shown that there was no correlation between HR, MR and AIx, and therefore highlights the likelihood of other mechanisms, beyond peripheral vasodilatation, by which HR increases in response to adenosine; such as action on the sympathetic nervous system ${ }^{139}$. The lack of correlation between HR and peripheral vasodilatation could also be explained by variable peripheral vasodilatory responses and known variations within adenosine receptor signalling pathways across individuals ${ }^{139,140}$.

Even in the presence of a significant HR increase, mean peak HR and mean lowest $\mathrm{Pd} / \mathrm{Pa}$ occurred at different times (Figure 5). If peripheral vasodilation is the major determinant of HR increases, 
this response may be captured at a different time frame to that of maximal coronary vasodilatation and it could be inferred that complete saturation of the coronary and peripheral vascular beds may be occurring at separate times during adenosine infusion. This is supported by the fact that adenosine receptors have been found to vary in affinity for drugs across different vascular beds ${ }^{141,142}$.

If $\mathrm{HR}$ is an unreliable surrogate of hyperaemia then what other options exist in non-invasive settings, such as CMR? Our data suggests that SBP or RPP changes are similarly unreliable indices of hyperaemia (Figure 6). Another option is to focus on non-haemodynamic markers of hyperaemia such as splenic blood flow attenuation, which can be assessed during a single breathhold without the need for gadolinium and may be a more reliable marker of coronary hyperaemia compared to classical haemodynamic markers such as change in $\mathrm{HR}$ or $\mathrm{SBP}^{143}$. Another potential method of limiting the HR variability is to consider further investigating vasodilator agents with more selective A2A-receptor action within the non-invasive imaging setting, such as Regadenoson $^{144}$.

Our study has demonstrated that assessment of hyperaemia in the cardiac catheterisation laboratory should rely on a combination of several invasive pressure-waveform based indices. In the non-invasive CMR setting, we demonstrate that HR and other haemodynamic surrogates are unreliable markers of hyperaemia, owing perhaps to variability in dose responses in different vascular beds. Although our study did not specifically evaluate variability in symptoms during adenosine-induced hyperaemia, anecdotal variability, e.g. chest tightness, breathlessness and flushing, are also likely to be a result of variability in adenosine receptor responses in different vascular beds. Whilst we appreciate that symptomatic changes are important to assess, due to the retrospective nature of the study, we could not assess these in a standardised way. Perhaps assessment of adenosine-induced symptoms in combination with surrogate non-invasive indices would provide a better method for detecting hyperaemia in patients receiving IV adenosine until alternative indices are developed. 


\section{Study Limitations}

This is a retrospectively analysed, heterogeneous cohort of patients who may have had different levels of pre-medication (including sedation), beta-blockade, tobacco smoking and caffeine intake prior to the catheter laboratory visit, confounding their responses to adenosine. However, at our institute, patients are advised to abstain from caffeine and anti-anginal medication, especially betablockers, prior to catheter laboratory tests.

Whilst an increase in coronary blood flow as determined by Doppler (CFR) is the gold standard for assessing hyperaemia within the Catheter Laboratory, an absence of flow augmentation does not necessarily imply inadequate hyperaemic stimulus. This is partly because CFR is dependent on the both epicardial vessels and microvasculature, hence may be an imperfect measure hyperaemia, for example in the context of microvascular coronary disease where it may be impossible to distinguish inadequate hyperaemic stimulus from diminished responsiveness. In patients who truly are non-responsive to adenosine, a different stressor such as Dobutamine or physical exercise may be more appropriate.

\section{Conclusion}

An increase in HR has high PPV but poor NPV as a surrogate marker of coronary hyperaemia in response to IV adenosine. Even in patients who respond, the time to maximum HR is not always an indicator of maximal hyperaemia. In the cardiac catheterisation laboratory, hyperaemia is best adjudicated by assessing multiple coronary pressure waveform indices, including dicrotic notch disappearance, ventricularisation of the $\mathrm{Pd}$ trace and separation of $\mathrm{Pd}$ and $\mathrm{Pa}$ values, rather than reliance on one index alone. In the non-invasive setting, the high PPV of HR as a surrogate marker of hyperaemia can be helpful, when no other reliable physiologic parameters are available, but should be interpreted cautiously. Further research is needed to develop optimal methods for identifying coronary hyperaemia outside the catheter laboratory. 
APPENDIX B: ETHICS APPROVAL, CONSENT FORM AND PATIENT INFORMATION SHEETS 


\section{B1: ETHICS APPROVAL LETTERS}

\section{Initial Ethical Approval (2016)}

\section{WHS \\ Health Research Authority \\ London - London Bridge Research Ethics Committee \\ Skipton House \\ 80 London Road \\ London \\ SE1 6LH \\ Telephone: 02079722580}

14 January 2016

Dr Divaka Perera

Department of Cardiology, St Thomas' Hospital

London, SE1 7EH

Dear Dr Perera

Study title:

Optimising Management of Serial and Diffuse Coronary

Artery Disease

REC reference:

15/LO/2011

Protocol number:

IRAS project ID:

1

191294

Thank you for your letter of 5 January 2016, responding to the Committee's request for further information on the above research and submitting revised documentation.

The further information was considered in correspondence by a Sub-Committee of the REC. A list of the Sub-Committee members is attached.

We plan to publish your research summary wording for the above study on the HRA website, together with your contact details. Publication will be no earlier than three months from the date of this opinion letter. Should you wish to provide a substitute contact point, require further information, or wish to make a request to postpone publication, please contact the REC Manager, Miss Kirstie Shearman, nrescommittee.london-londonbridge@nhs.net.

\section{Confirmation of ethical opinion}

On behalf of the Committee, I am pleased to confirm a favourable ethical opinion for the above research on the basis described in the application form, protocol and supporting documentation as revised, subject to the conditions specified below.

\section{Conditions of the favourable opinion}

The REC favourable opinion is subject to the following conditions being met prior to the start of the study.

Management permission must be obtained from each host organisation prior to the start of the study at the site concerned. 
Management permission should be sought from all NHS organisations involved in the study in accordance with NHS research governance arrangements. Each NHS organisation must confirm through the signing of agreements and/or other documents that it has given permission for the research to proceed (except where explicitly specified otherwise).

Guidance on applying for NHS permission for research is available in the Integrated Research Application System, www.hra.nhs.uk or at http://www.rdforum.nhs.uk.

Where a NHS organisation's role in the study is limited to identifying and referring potential participants to research sites ("participant identification centre"), guidance should be sought from the $R \& D$ office on the information it requires to give permission for this activity.

For non-NHS sites, site management permission should be obtained in accordance with the procedures of the relevant host organisation.

Sponsors are not required to notify the Committee of management permissions from host organisations

\section{Registration of Clinical Trials}

All clinical trials (defined as the first four categories on the IRAS filter page) must be registered on a publically accessible database within 6 weeks of recruitment of the first participant (for medical device studies, within the timeline determined by the current registration and publication trees).

There is no requirement to separately notify the REC but you should do so at the earliest opportunity e.g. When submitting an amendment. We will audit the registration details as part of the annual progress reporting process.

To ensure transparency in research, we strongly recommend that all research is registered but for non-clinical trials this is not currently mandatory.

If a sponsor wishes to contest the need for registration they should contact Catherine Blewett (catherineblewett@nhs.net), the HRA does not, however, expect exceptions to be made. Guidance on where to register is provided within IRAS.

It is the responsibility of the sponsor to ensure that all the conditions are complied with before the start of the study or its initiation at a particular site (as applicable).

\section{Ethical review of research sites}

NHS sites

The favourable opinion applies to all NHS sites taking part in the study, subject to management permission being obtained from the NHS/HSC R\&D office prior to the start of the study (see "Conditions of the favourable opinion" below).

Non-NHS sites

Approved documents 
The final list of documents reviewed and approved by the Committee is as follows:

\begin{tabular}{|l|l|l|}
\hline Document & Version & Date \\
\hline Covering letter on headed paper [Covering Letter] & 1 & 05 January 2016 \\
\hline $\begin{array}{l}\text { Evidence of Sponsor insurance or indemnity (non NHS Sponsors } \\
\text { only) [KCL Indemnity] }\end{array}$ & 1 & 15 July 2015 \\
\hline GP/consultant information sheets or letters [GP Notification Letter] & 1 & 05 January 2016 \\
\hline Letter from funder [BHF CRTF AWARD BM 2015] & 1 & 05 January 2016 \\
\hline Letter from statistician [Statistics Power Calculation] & 1 & 05 January 2016 \\
\hline Other [12h Notice Writing] & 1 & 05 January 2016 \\
\hline Other [Patient Copy of Results in Writing] & 1 & 05 January 2016 \\
\hline Participant consent form [FFR Pullback 2015 Consent_form_V4] & 4 & 05 January 2016 \\
\hline Participant information sheet (PIS) [Pt_info_sheet_FFR Pullback v6] & 6 & 05 January 2016 \\
\hline REC Application Form [REC_Form_03112015] & & 03 November 2015 \\
\hline $\begin{array}{l}\text { Referee's report or other scientific critique report [Radiation Safety } \\
\text { Report] }\end{array}$ & 1 & 19 October 2015 \\
\hline Research protocol or project proposal [Protocol] & 5 & 30 October 2015 \\
\hline Summary CV for Chief Investigator (CI) [Perera Short CV] & 1 & 19 October 2015 \\
\hline Summary CV for student [Short CV Modi] & 1 & \\
\hline
\end{tabular}

\section{Statement of compliance}

The Committee is constituted in accordance with the Governance Arrangements for Research Ethics Committees and complies fully with the Standard Operating Procedures for Research Ethics Committees in the UK.

\section{After ethical review}

\section{$\underline{\text { Reporting requirements }}$}

The attached document "After ethical review - guidance for researchers" gives detailed guidance on reporting requirements for studies with a favourable opinion, including:

- Notifying substantial amendments

- Adding new sites and investigators

- Notification of serious breaches of the protocol

- Progress and safety reports

- Notifying the end of the study

The HRA website also provides guidance on these topics, which is updated in the light of changes in reporting requirements or procedures.

\section{User Feedback}

The Health Research Authority is continually striving to provide a high quality service to all applicants and sponsors. You are invited to give your view of the service you have received and the application procedure. If you wish to make your views known please use the feedback form 
available on the HRA website:

http://www.hra.nhs.uk/about-the-hra/governance/quality-assurance/

\section{HRA Training}

We are pleased to welcome researchers and R\&D staff at our training days - see details at http://www.hra.nhs.uk/hra-training/

15/LO/2011 Please quote this number on all correspondence

With the Committee's best wishes for the success of this project.

Yours sincerely

pp.

\section{Kohearman}

Professor David Bartlett

Chair

Email:nrescommittee.london-londonbridge@nhs.net

Enclosures: $\quad$ List of names and professions of members who were present at the meeting and those who submitted written comments

"After ethical review - guidance for researchers"

Copy to:

Mr Keith Brennan

Jennifer Boston, Guy's \& St Thomas' Foundation NHS Trust 


\section{London - London Bridge Research Ethics Committee}

Attendance at Sub-Committee of the REC meeting on 20 January 2016

\section{Committee Members:}

\begin{tabular}{|l|l|l|} 
Name & Profession & Present \\
\hline Professor David Bartlett (Chair) & Honorary Consultant & Yes \\
\hline Dr Ralph White & Pharmacist & Yes \\
\hline
\end{tabular}

Also in attendance:

Name

Position (or reason for attending)

Mr Ali Hussain

REC Assistant 


\title{
Substantial Amendment 1 (co-recruitment), 2017
}

\author{
NHS \\ Health Research Authority \\ London - London Bridge Research Ethics Committee \\ Skipton House \\ 80 London Road \\ London \\ SE1 6LH
}

Tel: 02071048208

\author{
Please note: This is the \\ favourable opinion of the REC \\ only and does not allow the \\ amendment to be implemented \\ at NHS sites in England until \\ the outcome of the HRA \\ assessment has been \\ confirmed.
}

02 May 2017

Dr Divaka Perera

The Rayne Institute, 4th Floor Lambeth Wing

St Thomas' Hospital

Westminster Bridge Road

SE1 7EH

Dear Dr Perera

Study title:

Optimising Management of Serial and Diffuse Coronary

REC reference: Artery Disease

Protocol number:

Amendment number:

Amendment date:

IRAS project ID:

15/LO/2011

1

1

15 February 2017

191294

The above amendment was reviewed by the Sub-Committee in correspondence.

\section{Ethical opinion}

The members of the Committee taking part in the review gave a favourable ethical opinion of the amendment on the basis described in the notice of amendment form and supporting documentation

The Sub-Committee reviewed this amendment and requested to see the IRAS Form for 15/LO/1589.

You provided various versions of $15 / \mathrm{LO} / 1589$ before and after it was amended. The SubCommittee also reviewed the IRAS Form for 15/LO/2011.

The Sub-Committee were subsequently satisfied with the amendment.

Approved documents

A Research Ethics Committee established by the Health Research Authority 
The documents reviewed and approved at the meeting were:

\begin{tabular}{|l|l|l|}
\hline Document & Version & Date \\
\hline Notice of Substantial Amendment (non-CTIMP) & 1 & 15 February 2017 \\
\hline Notice of Substantial Amendment (non-CTIMP) [15/LO/1589] & 1 & 15 February 2017 \\
\hline Other [REC Form 15/LO/2011] & & 03 November 2015 \\
\hline Other [FO of SA 1 - 15/LO/1589] & & 11 March 2017 \\
\hline Other [FO 15/LO/1589] & & 20 October 2015 \\
\hline Other [REC Form 15/LO/1589] & & 24 August 2015 \\
\hline
\end{tabular}

\section{Membership of the Committee}

The members of the Committee who took part in the review are listed on the attached sheet.

\section{Working with NHS Care Organisations}

Sponsors should ensure that they notify the R\&D office for the relevant NHS care organisation of this amendment in line with the terms detailed in the categorisation email issued by the lead nation for the study.

\section{Statement of compliance}

The Committee is constituted in accordance with the Governance Arrangements for Research Ethics Committees and complies fully with the Standard Operating Procedures for Research Ethics Committees in the UK.

We are pleased to welcome researchers and R \& D staff at our Research Ethics Committee members' training days - see details at http://www.hra.nhs.uk/hra-training/

\section{5/LO/2011: $\quad$ Please quote this number on all correspondence}

Yours sincerely

PP:

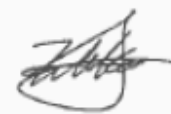

\section{Dr Michael Goggin}

Chair

E-mail: nrescommittee.london-londonbridge@nhs.net

Enclosures: $\quad$ List of names and professions of members who took part in the review

Copy to: $\quad$ Mrs Jen Boston, Guy's and St Thomas' NHS Foundation Trust Keith Brennan, King's College London 


\section{London - London Bridge Research Ethics Committee}

\section{Attendance at Sub-Committee of the REC meeting held in correspondence}

\section{Committee Members:}

\begin{tabular}{|l|l|l|l|}
\hline Name & Profession & Present & Notes \\
\hline Dr Frances Dockery & Consultant Physician & Yes & \\
\hline Dr Michael Goggin - Chair & Consultant Physician & Yes & \\
\hline
\end{tabular}

Also in attendance:

\begin{tabular}{|l|l|} 
Name & Position (or reason for attending) \\
\hline Ms Zuleka Alawi & REC Assistant \\
\hline
\end{tabular}




\section{Substantial Amendment 2 (FFR CT $_{\text {analysis), } 2018}$}

\section{NHS}

Health Research

Authority

London - London Bridge Research Ethics Committee

Skipton House

80 London Road

London

SE1 6LH

Tel: 02071048222

Fax: 02071048222

\section{Please note: This is the \\ favourable opinion of the REC \\ only and does not allow the \\ amendment to be implemented \\ at NHS sites in England until \\ the outcome of the HRA \\ assessment has been \\ confirmed.}

09 March 2018

Dr Bhavik Modi

15b Denholme Road

London

London

B77 4JP

Dear Dr Modi

Study title:

REC reference:

Optimising Management of Serial and Diffuse Coronary Artery Disease

Protocol number: 15/LO/2011

IRAS pi

12 February 2018

191294

The above amendment was reviewed by the Sub-Committee in correspondence.

\section{Ethical opinion}

The members of the Committee taking part in the review gave a favourable ethical opinion of the amendment on the basis described in the notice of amendment form and supporting documentation.

\section{Discussion}




\section{B2: CONSENT FORM \& PATIENT INFORMATION SHEETS}

\section{Guy's and St Thomas' W/ES \\ NHS Foundation Trust}

Participant Consent Form

REC Study Number:

\section{CONSENT FORM}

Version 5

Date 09.02.2018

Title of Project:

Optimising Management of Serial and Diffuse Coronary Artery Disease

Name of Researcher:

Name of Principal Investigator:
Dr. Bhavik Modi

Prof. Divaka Perera

\section{Please initial box}

1. I confirm that I have read and understand the Patient Information Sheet for ' Optimising Management of Serial and Diffuse Coronary Artery Disease' (Version 7 dated 09.02.2018) for the above study and have had the opportunity to ask questions.

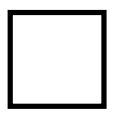

2. I understand that my participation is voluntary and that I am free to withdraw at any time, without giving any reason, and without my medical care or legal rights being affected.

3. I understand that sections of any of my medical notes may be looked at by responsible individuals from regulatory authorities where it is relevant to my taking part in research. I give permission for these individuals to have access to my records.

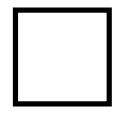

4. I agree to take part in the above study.

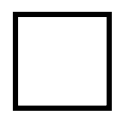

5 I agree to my General Practitioner being informed of my participation in this study

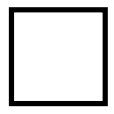

6. I agree that my CT data will be anonymised, sent to and analysed by a 3rd party company, based in the United States, for the purpose of this research study

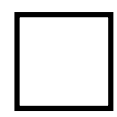

Name of Participant: Date: Signature:

Name of Person Consenting: Date: Signature: 


\section{Guy's and St Thomas' WHS \\ NHS Foundation Trust}

You are being invited to take part in a research study. Before you decide it is important for you to understand why the research is being done and what it will involve. Please take your time to read the following information carefully. Ask us if there is anything that is not clear or if you would like more information.

\section{'Optimising Management of Serial and Diffuse Coronary Artery Disease'} (REC Study No: 15/LO/2011)

\section{Why is study being carried out?}

Angina and Heart Attacks are caused by narrowings within blood vessels supplying the heart. Individual narrowings that need treatment are usually identified by making an educated guess of the effect that each will have on blood flow, based on the appearance of the artery on angiography (an invasive x-ray procedure which involves injecting dye into each heart artery). However, we can now accurately determine the effect of a narrowing on blood flow, using a measurement called Fractional Flow Reserve (FFR) during the angiography using a special angioplasty wire placed within the heart artery.

Treatment based on FFR yields better outcomes, but FFR is less useful in patients who have multiple narrowings in an artery - a common clinical problem. This study will investigate new techniques for this scenario of multiple narrowings. One of these techniques is a modification of the angiogram and angioplasty assessment that you have already been scheduled for and the other is a modification of the data from a Cardiac CT Scan, which you may already have had.

\section{Who is taking part in the study?}

We are inviting patients who have suspected coronary artery disease, who are awaiting coronary angiography and potential stenting at St Thomas' Hospital.

\section{Do I have to take part?}

No, it is entirely voluntary to take part in this study. If you do, you will be given this information sheet to keep and be asked to sign a consent form. You are still free to withdraw at any time and without giving a reason. A decision to withdraw at any time, or a decision not to take part, will not affect the standard of care you receive.

\section{Will I definitely be included in the study if I agree to take part?}

No. If the pattern of disease we find during your angiogram is not appropriate, you may not be included in the study even if you have agreed to take part.

\section{What is involved if I say "YES"?}

At present, as part of your normal care, you are scheduled to have a coronary angiogram with the possibility of pressure recordings made within narrowed arteries (using a special angioplasty wire) to help with choosing the best treatment option (options include stents, medicines, operation).

If you consent to the study and your angiogram shows an appropriate pattern of serial/diffuse coronary artery disease, you may have additional recordings made using a special angioplasty wire (in addition to the pressure recordings made within and narrowed heart arteries as part of 
your normal care). You may be asked to have a cardiac CT scan prior to having the angiogram (if not already performed as part of your normal care).

Participation is entirely voluntary and deciding not to participate will not affect the standard of care that you will receive.

\section{What are the potential risks and disadvantages of taking part?}

As part of your normal care, you will have a coronary angiogram and pressure recordings made with a special angioplasty wire during an infusion of a drug called adenosine. Adenosine is a very safe drug used to dilate the blood vessels of the heart but can occasionally produce very short-lived sensations of flushing and chest tightness. If you agree to being part of this study, we will use a modified angioplasty wire (already used in clinical practice for other purposes) to make additional recordings to normal care during which we will also be using adenosine. In taking these additional measurements, the catheter laboratory procedure will be lengthened by about 5 to 10 minutes with a small (no more than 1-2 minutes) increase in time exposed to the adenosine infusion but with no significant increase in the risk of the procedure.

The CT scan, if we decide we need one from you as part of the study and if you have not already had one, will last for approximately 30 minutes. CT Imaging is safe but does involve exposure to a small dose potentially harmful X-rays. Some patients may find the scanner uncomfortable and potentially claustrophobic.

As the catheter laboratory procedure will be lengthened by about 5 to 10 minutes compared to normal, there is a small additional radiation exposure. The total amount of radiation you will be exposed to will not exceed $71 \mathrm{mSv}$ (milliSievert). This corresponds to about 32 years of natural background radiation. Of this total amount of radiation the part that is extra to normal treatment could be up to $28.3 \mathrm{mSv}$, or roughly 13 years of natural background radiation. In most cases though, the dose that is extra to normal treatment will not exceed $1.3 \mathrm{mSv}$ - or 7 months of natural background radiation. All the doses here represent upper estimates, and the typical dose is anticipated to be approximately one third of these values. Background radiation is the amount of naturally occurring radiation in the environment coming from the earth itself and the suns rays

\section{What are the possible benefits of taking part?}

There are no direct benefits from taking part in this study over the usual standard clinical care of your condition. We will obtain very useful information from taking the extra measurements in the catheter laboratory using the modified specialist angioplasty wires and also the CT scan (should it be required and you have not already had one). This information we get might help improve the treatment of patients with coronary artery disease in the future. You will not be paid for participating in the trial.

\section{What if there is a problem?}

If you have a concern about any aspect of this study, you should ask to speak to the researchers who will do their best to answer your questions [Principal Investigator: Dr Divaka Perera, Divaka.Perera@kcl.ac.uk, 02071881048].

If you remain unhappy and wish to complain formally, you can do this through the Guy's and St Thomas' Patients Advice and Liaison Service (PALS) on 0207188 8801, pals@gstt.nhs.uk. The PALS team are based in the main entrance on the ground floor at St Thomas' Hospital and on the ground floor at Guy's Hospital in the Tower Wing. In the event that something does go wrong and you are harmed during the research you may have grounds for legal action for compensation against Guy's and St Thomas' NHS Foundation Trust and/or King's College London but you may have to pay your legal costs. The normal National Health Service complaints mechanisms will still be available to you (if appropriate).

\section{What will happen if I don't want to carry on with the study?}


You can withdraw from the study at any time without giving any reason. All identifiable data will be destroyed and only unidentifiable data may be retained.

\section{Will my taking part in this study be kept confidential?}

All the information that is collected about you during the course of this study will be kept strictly confidential. The anonymous data may be used in the future for further studies. The data collected from the CT Scan will be also anonymised and sent to a $3^{\text {rd }}$ party company (Heartflow) in the United States for analysis for the purpose of research.

\section{Contact numbers:}

- Dr Bhavik Modi, Research Registrar, Dept of Cardiology, St Thomas' Hospital, bhavikmodi@hotmail.com; +44 (0) 207188 3026; +44 7931400880

- Prof Divaka Perera, Consultant Cardiologist, St Thomas’ Hospital

- Prof Michael Marber, Dept of Cardiology, The Rayne Institute, St Thomas' Hospital, London SE1 7EH, Tel: +44(0)20-7188-1008, Fax +44(0)20-7188-097

This study is funded via a BHF Fellowship Grant and the study will go towards the work of a clinical PhD project. The study has been reviewed by The London Bridge Research Ethics Committee 


\section{REFERENCES}

1. Toth G, Hamilos M, Pyxaras S, et al. Evolving concepts of angiogram: fractional flow reserve discordances in 4000 coronary stenoses. Eur Heart J. 2014;35(40):2831-2838. doi:10.1093/eurheartj/ehu094.

2. Bech GJW, De Bruyne B, Pijls NHJ, et al. Fractional Flow Reserve to Determine the Appropriateness of Angioplasty in Moderate Coronary Stenosis : A Randomized Trial. Circulation. 2001;103(24):2928-2934. doi:10.1161/01.CIR.103.24.2928.

3. Tonino PAL, De Bruyne B, Pijls NHJ, et al. Fractional Flow Reserve versus Angiography for Guiding Percutaneous Coronary Intervention. http://dxdoiorg/101056/NEJMoa0807611. 2009;360(3):213-224. doi:10.1056/NEJMoa0807611.

4. De Bruyne B, Pijls NHJ, Kalesan B, et al. Fractional Flow Reserve-Guided PCI versus Medical Therapy in Stable Coronary Disease. New England Journal of Medicine. 2012;367(11):991-1001. doi:10.1056/NEJMoa1205361.

5. Dortimer AC, Shenoy PN, Shiroff RA, et al. Diffuse coronary artery disease in diabetic patients: fact or fiction? Circulation. 1978;57(1):133-136.

6. Di Sciascio G, Patti G, Nasso G, Manzoli A, D'Ambrosio A, Abbate A. Early and longterm results of stenting of diffuse coronary artery disease. AJC. 2000;86(11):1166-1170.

7. Hachamovitch R. Comparison of the Short-Term Survival Benefit Associated With Revascularization Compared With Medical Therapy in Patients With No Prior Coronary Artery Disease Undergoing Stress Myocardial Perfusion Single Photon Emission Computed Tomography. Circulation. 2003;107(23):2900-2907. doi:10.1161/01.CIR.0000072790.23090.41.

8. Pijls NHJ, De Bruyne B, Peels K, et al. Measurement of Fractional Flow Reserve to Assess the Functional Severity of Coronary-Artery Stenoses. New England Journal of Medicine. 1996;334(26):1703-1708. doi:10.1056/NEJM199606273342604.

9. Curzen N, Rana O, Nicholas Z, et al. Does routine pressure wire assessment influence management strategy at coronary angiography for diagnosis of chest pain?: the RIPCORD study. Circulation: Cardiovascular Interventions. 2014;7(2):248-255. doi:10.1161/CIRCINTERVENTIONS.113.000978.

10. Tonino PAL, De Bruyne B, Pijls NHJ, et al. Fractional Flow Reserve versus Angiography for Guiding Percutaneous Coronary Intervention. New England Journal of Medicine. 2009;360(3):213-224. doi:10.1056/NEJMoa0807611.

11. Davies JE, Sen S, Dehbi H-M, et al. Use of the Instantaneous Wave-free Ratio or Fractional Flow Reserve in PCI. N Engl J Med. March 2017:NEJMoa1700445. doi:10.1056/NEJMoa1700445.

12. Götberg M, Christiansen EH, Gudmundsdottir IJ, et al. Instantaneous Wave-free Ratio versus Fractional Flow Reserve to Guide PCI. $N$ Engl J Med. March 2017:NEJMoa1616540. doi:10.1056/NEJMoa1616540.

13. Layland J, Oldroyd KG, Curzen N, et al. Fractional flow reserve vs. angiography in guiding management to optimize outcomes in non-ST-segment elevation myocardial 
infarction: the British Heart Foundation FAMOUS-NSTEMI randomized trial. Eur Heart J. 2015;36(2):100-111. doi:10.1093/eurheartj/ehu338.

14. Anderson HV, Roubin GS, Leimgruber PP, et al. Measurement of transstenotic pressure gradient during percutaneous transluminal coronary angioplasty. Circulation. 1986;73(6):1223-1230.

15. Jeremias A, Maehara A, Généreux P, et al. Multicenter Core Laboratory Comparison of the Instantaneous Wave-Free Ratio and Resting $\mathrm{Pd} / \mathrm{Pa}$ With Fractional Flow Reserve. Journal of the American College of Cardiology. 2014;63(13):1253-1261. doi:10.1016/j.jacc.2013.09.060.

16. Johnson NP, Kirkeeide RL, Asrress KN, et al. Does the Instantaneous Wave-Free Ratio Approximate the Fractional Flow Reserve? Journal of the American College of Cardiology. 2013;61(13):1428-1435. doi:10.1016/j.jacc.2012.09.064.

17. Kobayashi Y, Johnson NP, Zimmermann FM, et al. Agreement of the Resting Distal to Aortic Coronary Pressure With the Instantaneous Wave-Free Ratio. Journal of the American College of Cardiology. 2017;70(17):2105-2113. doi:10.1016/j.jacc.2017.08.049.

18. Lee JM, Choi KH, Park J, et al. Physiological and Clinical Assessment of Resting Physiological Indexes. Circulation. 2019;139(7):889-900.

doi:10.1161/CIRCULATIONAHA.118.037021.

19. van't Veer M, Pijls NHJ, Hennigan B, et al. Comparison of Different Diastolic Resting Indexes to iFR: Are They All Equal? Journal of the American College of Cardiology. 2017;70(25):3088-3096. doi:10.1016/j.jacc.2017.10.066.

20. van de Hoef TP, van Lavieren MA, Damman P, et al. Physiological basis and long-term clinical outcome of discordance between fractional flow reserve and coronary flow velocity reserve in coronary stenoses of intermediate severity. Circulation:

Cardiovascular Interventions. 2014;7(3):301-311.

doi:10.1161/CIRCINTERVENTIONS.113.001049.

21. Meuwissen M, Siebes M, Chamuleau SAJ, et al. Hyperemic stenosis resistance index for evaluation of functional coronary lesion severity. Circulation. 2002;106(4):441-446.

22. van de Hoef TP, Meuwissen M, Escaned J, et al. Head-to-head comparison of basal stenosis resistance index, instantaneous wave-free ratio, and fractional flow reserve: diagnostic accuracy for stenosis-specific myocardial ischaemia. EuroIntervention. 2015;11(8):914-925. doi:10.4244/EIJY14M08_17.

23. Gould KL. Pressure-flow characteristics of coronary stenoses in unsedated dogs at rest and during coronary vasodilation. Circulation Research. 1978;43(2):242-253. doi:10.1161/01.RES.43.2.242.

24. Boulpaep EL. ORGANIZATION OF THE CARDIOVASCULAR SYSTEM. In: Medical Physiology. Elsevier; 2009:429-447. doi:10.1016/B978-1-4160-3115-4.500202 .

25. Bernad SI, Bernad ES, Totorean AF, Craina ML, Sargan I. Clinical important hemodynamic characteristics for serial stenosed coronary artery. Brebbia CA, ed. International Journal of Design \& Nature and Ecodynamics. 2015;10(2):97-113. doi:10.2495/DNE-V10-N2-97-113. 
26. Uren NG, Melin JA, De Bruyne B, Wijns W, Baudhuin T, Camici PG. Relation between myocardial blood flow and the severity of coronary-artery stenosis. New England Journal of Medicine. 1994;330(25):1782-1788. doi:10.1056/NEJM199406233302503.

27. De Bruyne B, Pijls NH, Heyndrickx GR, Hodeige D, Kirkeeide R, Gould KL. Pressurederived fractional flow reserve to assess serial epicardial stenoses: theoretical basis and animal validation. Circulation. 2000;101(15):1840-1847.

28. Shalman E, Einav S. A method for estimating the physiological significance of each of serial vascular lesions. IEMBS-01. 2001;1:89-90vol.1.

doi:10.1109/IEMBS.2001.1018853.

29. Kern MJ, Seto AH. A Perspective on Physiologic Assessment of Coronary Stenoses in Series: Methods, Myths, and Best Practices? JAMA Cardiology. March 2018. doi:10.1001/jamacardio.2018.0239.

30. Pijls NHJ, De Bruyne B, Bech GJW, et al. Coronary Pressure Measurement to Assess the Hemodynamic Significance of Serial Stenoses Within One Coronary Artery : Validation in Humans. Circulation. 2000;102(19):2371-2377. doi:10.1161/01.CIR.102.19.2371.

31. Wolfrum M, Fahrni G, De Maria GL, et al. Impact of impaired fractional flow reserve after coronary interventions on outcomes: a systematic review and meta-analysis. BMC Cardiovascular Disorders 2016 16:1. 2016;16(1):177. doi:10.1186/s12872-016-0355-7.

32. Park SJ, Ahn JM, Pijls NHJ, et al. Validation of Functional State of Coronary Tandem Lesions Using Computational Flow Dynamics. AJC. 2012;110(11):1578-1584. doi:10.1016/j.amjcard.2012.07.023.

33. Kim HL, Koo BK, Nam CW, et al. Clinical and Physiological Outcomes of Fractional Flow Reserve-Guided Percutaneous Coronary Intervention in Patients With Serial Stenoses Within One Coronary Artery. JACC Cardiovasc Interv. 2012;5(10):10131018. doi:10.1016/j.jcin.2012.06.017.

34. Gould KL, Lipscomb K, Hamilton GW. Physiologic basis for assessing critical coronary stenosis. Instantaneous flow response and regional distribution during coronary hyperemia as measures of coronary flow reserve. AJC. 1974;33(1):87-94.

35. Nijjer SS, Sen S, Petraco R, et al. Pre-angioplasty instantaneous wave-free ratio pullback provides virtual intervention and predicts hemodynamic outcome for serial lesions and diffuse coronary artery disease. JACC Cardiovasc Interv. 2014;7(12):13861396. doi:10.1016/j.jcin.2014.06.015.

36. MD YK, MBBS CMC, MD ASPS, et al. Pre-Angioplasty Instantaneous Wave-Free Ratio Pullback Predicts Hemodynamic Outcome In Humans With\&nbsp;Coronary Artery Disease. JCIN. 2018;11(8):757-767. doi:10.1016/j.jcin.2018.03.005.

37. Lindstaedt M, Spiecker M, Perings C, et al. How good are experienced interventional cardiologists at predicting the functional significance of intermediate or equivocal left main coronary artery stenoses? Int J Cardiol. 2007;120(2):254-261. doi:10.1016/j.ijcard.2006.11.220.

38. Hamilos M, Muller O, Cuisset T, et al. Long-term clinical outcome after fractional flow reserve-guided treatment in patients with angiographically equivocal left main coronary artery stenosis. Circulation. 2009;120(15):1505-1512.

doi:10.1161/CIRCULATIONAHA.109.850073. 
39. Mallidi J, Atreya AR, Cook J, et al. Long-term outcomes following fractional flow reserve-guided treatment of angiographically ambiguous left main coronary artery disease: A meta-analysis of prospective cohort studies. Catheter Cardiovasc Interv. 2015;86(1):12-18. doi:10.1002/ccd.25894.

40. Leone AM, De Caterina AR, Basile E, et al. Influence of the amount of myocardium subtended by a stenosis on fractional flow reserve. Circulation: Cardiovascular Interventions. 2013;6(1):29-36. doi:10.1161/CIRCINTERVENTIONS.112.971101.

41. Daniels DV, van't Veer M, Pijls NHJ, et al. The impact of downstream coronary stenoses on fractional flow reserve assessment of intermediate left main disease. JACC Cardiovasc Interv. 2012;5(10):1021-1025. doi:10.1016/j.jcin.2012.07.005.

42. Spaan JAE. Physiological Basis of Clinically Used Coronary Hemodynamic Indices. Circulation. 2006;113(3):446-455. doi:10.1161/CIRCULATIONAHA.105.587196.

43. Taylor HA, Deumite NJ, Chaitman BR, Davis KB, Killip T, Rogers WJ. Asymptomatic left main coronary artery disease in the Coronary Artery Surgery Study (CASS) registry. Circulation. 1989;79(6):1171-1179. doi:10.1161/01.CIR.79.6.1171.

44. Fearon WF, Yong AS, Lenders G, et al. The impact of downstream coronary stenosis on fractional flow reserve assessment of intermediate left main coronary artery disease: human validation. JACC Cardiovasc Interv. 2015;8(3):398-403. doi:10.1016/j.jcin.2014.09.027.

45. Yamamoto E, Saito N, Matsuo H, et al. Prediction of the true fractional flow reserve of left main coronary artery stenosis with concomitant downstream stenoses: in vitro and in vivo experiments. EuroIntervention. 2016;11(11):e1249-e1256. doi:10.4244/EIJV11I11A246.

46. Yong ASC, Daniels D, De Bruyne B, et al. Fractional Flow Reserve Assessment of Left Main Stenosis in the Presence of Downstream Coronary Stenoses. Circulation: Cardiovascular Interventions. 2013;6(2):161-165. doi:10.1161/CIRCINTERVENTIONS.112.000104.

47. Johnson NP, Johnson DT, Kirkeeide RL, et al. Repeatability of Fractional Flow Reserve Despite Variations in Systemic and Coronary Hemodynamics. JACC Cardiovasc Interv. 2015;8(8):1018-1027. doi:10.1016/j.jcin.2015.01.039.

48. Perera D, Biggart S, Postema P, et al. Right atrial pressure: can it be ignored when calculating fractional flow reserve and collateral flow index? Journal of the American College of Cardiology. 2004;44(10):2089-2091. doi:10.1016/j.jacc.2004.08.018.

49. Oemrawsingh PV. Intravascular Ultrasound Guidance Improves Angiographic and Clinical Outcome of Stent Implantation for Long Coronary Artery Stenoses: Final Results of a Randomized Comparison With Angiographic Guidance (TULIP Study). Circulation. 2002;107(1):62-67. doi:10.1161/01.CIR.0000043240.87526.3F.

50. Jasti V. Correlations Between Fractional Flow Reserve and Intravascular Ultrasound in Patients With an Ambiguous Left Main Coronary Artery Stenosis. Circulation. 2004;110(18):2831-2836. doi:10.1161/01.CIR.0000146338.62813.E7.

51. Kang S-J, Lee J-Y, Ahn J-M, et al. Validation of intravascular ultrasound-derived parameters with fractional flow reserve for assessment of coronary stenosis severity. Circulation: Cardiovascular Interventions. 2011;4(1):65-71. doi:10.1161/CIRCINTERVENTIONS.110.959148. 
52. la Torre Hernandez de JM, Hernández Hernandez F, Alfonso F, et al. Prospective application of pre-defined intravascular ultrasound criteria for assessment of intermediate left main coronary artery lesions results from the multicenter LITRO study. Journal of the American College of Cardiology. 2011;58(4):351-358. doi:10.1016/j.jacc.2011.02.064.

53. Park S-J, Ahn J-M, Kang S-J, et al. Intravascular ultrasound-derived minimal lumen area criteria for functionally significant left main coronary artery stenosis. JACC Cardiovasc Interv. 2014;7(8):868-874. doi:10.1016/j.jcin.2014.02.015.

54. Fassa A-A, Wagatsuma K, Higano ST, et al. Intravascular ultrasound-guided treatment for angiographically indeterminate left main coronary artery disease: a long-term follow-up study. Journal of the American College of Cardiology. 2005;45(2):204-211. doi:10.1016/j.jacc.2004.09.066.

55. Kubo T, Akasaka T, Shite J, et al. OCT Compared With IVUS in a Coronary Lesion Assessment. JACC: Cardiovascular Imaging. 2013;6(10):1095-1104. doi:10.1016/j.jcmg.2013.04.014.

56. Gonzalo N, Gonzalo N, Escaned J, et al. Morphometric assessment of coronary stenosis relevance with optical coherence tomography: a comparison with fractional flow reserve and intravascular ultrasound. Journal of the American College of Cardiology. 2012;59(12):1080-1089. doi:10.1016/j.jacc.2011.09.078.

57. Miller JM, Rochitte CE, Dewey M, et al. Diagnostic Performance of Coronary Angiography by 64-Row CT. New England Journal of Medicine. 2008;359(22):23242336. doi:10.1056/NEJMoa0806576.

58. Budoff MJ, Dowe D, Jollis JG, et al. Diagnostic performance of 64-multidetector row coronary computed tomographic angiography for evaluation of coronary artery stenosis in individuals without known coronary artery disease: results from the prospective multicenter ACCURACY (Assessment by Coronary Computed Tomographic Angiography of Individuals Undergoing Invasive Coronary Angiography) trial. Journal of the American College of Cardiology. 2008;52(21):1724-1732. doi:10.1016/j.jacc.2008.07.031.

59. SCOT-HEART Investigators, Newby DE, Adamson PD, et al. Coronary CT Angiography and 5-Year Risk of Myocardial Infarction. $N$ Engl J Med. 2018;379(10):924-933. doi:10.1056/NEJMoa1805971.

60. Taylor AJ, Cerqueira M, Hodgson JM, et al. ACCF/SCCT/ACR/AHA/ASE/ASNC/NASCI/SCAI/SCMR 2010 Appropriate Use Criteria for Cardiac Computed Tomography. Journal of the American College of Cardiology. 2010;56(22):1864-1894. doi:10.1016/j.jacc.2010.07.005.

61. Patel MR, Peterson ED, Dai D, et al. Low Diagnostic Yield of Elective Coronary Angiography. New England Journal of Medicine. 2010;362(10):886-895. doi:10.1056/NEJMoa0907272.

62. Taylor CA, Fonte TA, Min JK. Computational fluid dynamics applied to cardiac computed tomography for noninvasive quantification of fractional flow reserve: scientific basis. Journal of the American College of Cardiology. 2013;61(22):22332241. doi:10.1016/j.jacc.2012.11.083. 
63. Min JK, Taylor CA, Achenbach S, et al. Noninvasive Fractional Flow Reserve Derived From Coronary CT Angiography. JACC: Cardiovascular Imaging. 2015;8(10):12091222. doi:10.1016/j.jcmg.2015.08.006.

64. Rajani R, Modi B, Ntalas I, Curzen N. Non-invasive fractional flow reserve using computed tomographic angiography: where are we now and where are we going? Heart. 2017;103(15):1216-1222. doi:10.1136/heartjnl-2016-311029.

65. Koo B-K, Erglis A, Doh J-H, et al. Diagnosis of Ischemia-Causing Coronary Stenoses by Noninvasive Fractional Flow Reserve Computed From Coronary Computed Tomographic Angiograms. Journal of the American College of Cardiology. 2011;58(19):1989-1997. doi:10.1016/j.jacc.2011.06.066.

66. Min JK, Leipsic J, Pencina MJ, et al. Diagnostic Accuracy of Fractional Flow Reserve From Anatomic CT Angiography. JAMA. 2012;308(12):1237. doi:10.1001/2012.jama.11274.

67. Nørgaard BL, Leipsic J, Gaur S, et al. Diagnostic performance of noninvasive fractional flow reserve derived from coronary computed tomography angiography in suspected coronary artery disease: the NXT trial (Analysis of Coronary Blood Flow Using CT Angiography: Next Steps). Journal of the American College of Cardiology. 2014;63(12):1145-1155. doi:10.1016/j.jacc.2013.11.043.

68. Curzen NP, Nolan J, Zaman AG, Nørgaard BL, Rajani R. Does the Routine Availability of CT-Derived FFR Influence Management of Patients With Stable Chest Pain Compared to CT Angiography Alone?: The FFRCT RIPCORD Study. JACC: Cardiovascular Imaging. 2016;9(10):1188-1194. doi:10.1016/j.jcmg.2015.12.026.

69. Douglas PS, Pontone G, Hlatky MA, et al. Clinical outcomes of fractional flow reserve by computed tomographic angiography-guided diagnostic strategies vs. usual care in patients with suspected coronary artery disease: the prospective longitudinal trial of FFR(CT): outcome and resource impacts study. Eur Heart J. 2015;36(47):3359-3367. doi:10.1093/eurheartj/ehv444.

70. Douglas PS, De Bruyne B, Pontone G, et al. 1-Year Outcomes of FFR CT -Guided Care in Patients With Suspected Coronary Disease. Journal of the American College of Cardiology. 2016;68(5):435-445. doi:10.1016/j.jacc.2016.05.057.

71. Tanaka K, Bezerra HG, Gaur S, et al. Comparison Between Non-invasive (Coronary Computed Tomography Angiography Derived) and Invasive-Fractional Flow Reserve in Patients with Serial Stenoses Within One Coronary Artery: A NXT Trial substudy. Ann Biomed Eng. 2016;44(2):580-589. doi:10.1007/s10439-015-1436-y.

72. Agarwal SK, Raina S, Edupuganti M, et al. PREDICTIVE ACCURACY OF RESTING GRADIENT (PD/PA) FOR IDENTIFYING ISCHEMIC CORONARY LESIONS. Journal of the American College of Cardiology. 2016;67(13):380. doi:10.1016/S07351097(16)30381-3.

73. Sen S, Escaned J, Malik IS, et al. Development and Validation of a New AdenosineIndependent Index of Stenosis Severity From Coronary Wave-Intensity Analysis. Journal of the American College of Cardiology. 2012;59(15):1392-1402. doi:10.1016/j.jacc.2011.11.003.

74. Bech GJW, De Bruyne B, Pijls NHJ, et al. Fractional Flow Reserve to Determine the Appropriateness of Angioplasty in Moderate Coronary Stenosis : A Randomized Trial. Circulation. 2001;103(24):2928-2934. doi:10.1161/01.CIR.103.24.2928. 
75. Nijjer SS, Petraco R, van de Hoef TP, et al. Change in coronary blood flow after percutaneous coronary intervention in relation to baseline lesion physiology: results of the JUSTIFY-PCI study. Circulation: Cardiovascular Interventions. 2015;8(6):e001715-e001715. doi:10.1161/CIRCINTERVENTIONS.114.001715.

76. Biglino G, Verschueren P, Zegels R, Taylor AM, Schievano S. Rapid prototyping compliant arterial phantoms for in-vitro studies and device testing. Journal of Cardiovascular Magnetic Resonance. 2013;15(1):2. doi:10.1186/1532-429X-15-2.

77. Yamamoto E, Saito N, Matsuo H, et al. Prediction of the true fractional flow reserve of left main coronary artery stenosis with concomitant downstream stenoses: in vitro and in vivo experiments. EuroIntervention. 2016;11(11):e1249-e1256. doi:10.4244/EIJV11I11A246.

78. Mates RE, Gupta RL, Bell AC, Klocke FJ. Fluid dynamics of coronary artery stenosis. Circulation Research. 1978;42(1):152-162. doi:10.1161/01.RES.42.1.152.

79. Johnson NP, Jeremias A, Zimmermann FM, et al. Continuum of Vasodilator Stress From Rest to Contrast Medium to Adenosine Hyperemia for Fractional Flow Reserve Assessment. JACC Cardiovasc Interv. 2016;9(8):757-767. doi:10.1016/j.jcin.2015.12.273.

80. Gaddum NR, Alastruey J, Chowienczyk P, Rutten MCM, Segers P, Schaeffter T. Relative Contributions from the Ventricle and Arterial Tree to Arterial Pressure and its Amplification: An Experimental Study. Am J Physiol Heart Circ Physiol. June 2017:ajpheart.00844.2016. doi:10.1152/ajpheart.00844.2016.

81. Young DF. Fluid Mechanics of Arterial Stenoses. Journal of Biomechanical Engineering. 1979;101(3):157-175. doi:10.1115/1.3426241.

82. Manor D, Beyar R, Shofti R, Sideman S. In-vivo study of the mechanical properties of epicardial coronary arteries. Journal of Biomechanical Engineering. 1994;116(1):131132.

83. Fearon WF, Nishi T, De Bruyne B, et al. Clinical Outcomes and Cost-Effectiveness of Fractional Flow Reserve-Guided Percutaneous Coronary Intervention in Patients With Stable Coronary Artery Disease: Three-Year Follow-Up of the FAME 2 Trial (Fractional Flow Reserve Versus Angiography for Multivessel Evaluation). Circulation. November 2017:CIRCULATIONAHA.117.031907. doi:10.1161/CIRCULATIONAHA.117.031907.

84. Davies JE, Sen S, Dehbi H-M, et al. Use of the Instantaneous Wave-free Ratio or Fractional Flow Reserve in PCI. N Engl J Med. March 2017:NEJMoa1700445. doi:10.1056/NEJMoa1700445.

85. Dourado LOC, Bittencourt MS, Pereira AC, et al. Coronary Artery Bypass Surgery in Diffuse Advanced Coronary Artery Disease: 1-Year Clinical and Angiographic Results. Thorac Cardiovasc Surg. 2017;(EFirst). doi:10.1055/s-0037-1601306.

86. Modi BN, De Silva K, Rajani R, Curzen N, Perera D. Physiology-Guided Management of Serial Coronary Artery Disease. JAMA Cardiology. March 2018:1-7. doi:10.1001/jamacardio.2018.0236.

87. Sen S, Asrress KN, Nijjer S, et al. Diagnostic classification of the instantaneous wavefree ratio is equivalent to fractional flow reserve and is not improved with adenosine administration. Results of CLARIFY (Classification Accuracy of Pressure-Only Ratios 
Against Indices Using Flow Study). Journal of the American College of Cardiology. 2013;61(13):1409-1420. doi:10.1016/j.jacc.2013.01.034.

88. Modi BN, Rahman H, Sherif SA, et al. Is heart rate response a reliable marker of adenosine-induced coronary hyperemia? Int J Cardiovasc Imaging. 2018;107:29002909. doi:10.1007/s10554-018-1309-1.

89. Adjedj J, Toth GG, Johnson NP, et al. Intracoronary Adenosine: Dose-Response Relationship With Hyperemia. JACC Cardiovasc Interv. 2015;8(11):1422-1430. doi:10.1016/j.jcin.2015.04.028.

90. Schlundt C, Bietau C, Klinghammer L, et al. Comparison of intracoronary versus intravenous administration of adenosine for measurement of coronary fractional flow reserve. Circulation: Cardiovascular Interventions. 2015;8(5):e001781-e001781. doi:10.1161/CIRCINTERVENTIONS.114.001781.

91. van de Hoef TP, Petraco R, van Lavieren MA, et al. Basal stenosis resistance index derived from simultaneous pressure and flow velocity measurements. EuroIntervention. 2016;12(2):e199-e207. doi:10.4244/EIJV12I2A33.

92. Verhoeff B-J, Siebes M, Meuwissen M, et al. Influence of Percutaneous Coronary Intervention on Coronary Microvascular Resistance Index. Circulation. 2005;111(1):7682. doi:10.1161/01.CIR.0000151610.98409.2F.

93. Kanaji Y, Murai T, Yonetsu T, et al. Effect of Elective Percutaneous Coronary Intervention on Hyperemic Absolute Coronary Blood Flow Volume and Microvascular Resistance. Circulation: Cardiovascular Interventions. 2017;10(10):e005073. doi:10.1161/CIRCINTERVENTIONS.117.005073.

94. De Bruyne B, Pijls NHJ, Kalesan B, et al. Fractional Flow Reserve-Guided PCI versus Medical Therapy in Stable Coronary Disease. New England Journal of Medicine. 2012;367(11):991-1001. doi:10.1056/NEJMoa1205361.

95. Modi BN, De Silva K, Rajani R, Curzen N, Perera D. Physiology-Guided Management of Serial Coronary Artery Disease. JAMA Cardiology. March 2018:1-3. doi:10.1001/jamacardio.2018.0236.

96. Taylor CA, Gaur S, Leipsic J, et al. Effect of the ratio of coronary arterial lumen volume to left ventricle myocardial mass derived from coronary $\mathrm{CT}$ angiography on fractional flow reserve. Journal of Cardiovascular Computed Tomography. 2017;11(6):429-436. doi:10.1016/j.jcct.2017.08.001.

97. LeCun Y, Bengio Y, Hinton G. Deep learning. Nature. 2015;521(7553):436-444. doi:10.1038/nature14539.

98. Cook CM, Petraco R, Shun-Shin MJ, et al. Diagnostic Accuracy of Computed Tomography-Derived Fractional Flow Reserve : A Systematic Review. JAMA Cardiology. 2017;2(7):803-810. doi:10.1001/jamacardio.2017.1314.

99. Siebes M, Chamuleau SAJ, Meuwissen M, Piek JJ, Spaan JAE. Influence of hemodynamic conditions on fractional flow reserve: parametric analysis of underlying model. AJP: Heart and Circulatory Physiology. 2002;283(4):H1462-H1470. doi:10.1152/ajpheart.00165.2002. 
100. Götberg M, Christiansen EH, Gudmundsdottir IJ, et al. Instantaneous Wave-free Ratio versus Fractional Flow Reserve to Guide PCI. $N$ Engl J Med. March 2017:NEJMoa1616540. doi:10.1056/NEJMoa1616540.

101. Modi BN, Chattersingh A, Eruslanova K, et al. Abstract 17271: Optimising Physiological Assessment of Serial Coronary Artery Lesions Using an in vitro 3-D Printed Model of Tandem Stenoses. Circulation. 2017;136(Suppl 1):A17271.

102. Modi B, Perera D. 3 Optimising physiology guided management of serial coronary artery disease. In: Vol 104. BMJ Publishing Group Ltd and British Cardiovascular Society; 2018:A2-A3. doi:10.1136/heartjnl-2018-BCIS.3.

103. Modi BN, Chattersingh A, Eruslanova K, et al. Abstract 17271: Optimising Physiological Assessment of Serial Coronary Artery Lesions Using an in vitro 3-D Printed Model of Tandem Stenoses. Circulation. 2017;136(Suppl 1):A17271.

104. Patel MR, Calhoon JH, Dehmer GJ, et al.

ACC/AATS/AHA/ASE/ASNC/SCAI/SCCT/STS 2017 Appropriate Use Criteria for Coronary Revascularization in Patients With Stable Ischemic Heart Disease: A Report of the American College of Cardiology Appropriate Use Criteria Task Force, American Association for Thoracic Surgery, American Heart Association, American Society of Echocardiography, American Society of Nuclear Cardiology, Society for Cardiovascular Angiography and Interventions, Society of Cardiovascular Computed Tomography, and Society of Thoracic Surgeons. Journal of the American College of Cardiology. 2017;69(17):2212-2241. doi:10.1016/j.jacc.2017.02.001.

105. Kikuta Y, Cook CM, Sharp ASP, et al. Pre-Angioplasty Instantaneous Wave-Free Ratio Pullback Predicts Hemodynamic Outcome In Humans With Coronary Artery Disease: Primary Results of the International Multicenter iFR GRADIENT Registry. JACC Cardiovasc Interv. 2018;11(8):757-767. doi:10.1016/j.jcin.2018.03.005.

106. Dattilo PB, Prasad A, Honeycutt E, Wang TY, Messenger JC. Contemporary patterns of fractional flow reserve and intravascular ultrasound use among patients undergoing percutaneous coronary intervention in the United States: insights from the National Cardiovascular Data Registry. Journal of the American College of Cardiology. 2012;60(22):2337-2339. doi:10.1016/j.jacc.2012.08.990.

107. Bhatt DL. Assessment of Stable Coronary Lesions. New England Journal of Medicine. 2017;376(19):1879-1881. doi:10.1056/NEJMe1702728.

108. Tu S, Barbato E, Köszegi Z, et al. Fractional Flow Reserve Calculation From 3Dimensional Quantitative Coronary Angiography and TIMI Frame Count. JACC Cardiovasc Interv. 2014;7(7):768-777. doi:10.1016/j.jcin.2014.03.004.

109. Tu S, Westra J, Yang J, et al. Diagnostic Accuracy of Fast Computational Approaches to Derive Fractional Flow Reserve From Diagnostic Coronary Angiography. JACC Cardiovasc Interv. 2016;9(19):2024-2035. doi:10.1016/j.jcin.2016.07.013.

110. Johnson NP, Toth GG, Lai D, et al. Prognostic Value of Fractional Flow Reserve. Journal of the American College of Cardiology. 2014;64(16):1641-1654. doi:10.1016/j.jacc.2014.07.973.

111. Lockie T, Ishida M, Perera D, et al. High-resolution magnetic resonance myocardial perfusion imaging at 3.0-Tesla to detect hemodynamically significant coronary stenoses as determined by fractional flow reserve. Journal of the American College of Cardiology. 2011;57(1):70-75. doi:10.1016/j.jacc.2010.09.019. 
112. Boden WE, O'Rourke RA, Teo KK, et al. Optimal medical therapy with or without PCI for stable coronary disease. $N$ Engl J Med. 2007;356(15):1503-1516. doi:10.1056/NEJMoa070829.

113. Al-Lamee R, Thompson D, Dehbi H-M, et al. Percutaneous coronary intervention in stable angina (ORBITA): a double-blind, randomised controlled trial. Lancet. 2017;391(10115):31-40. doi:10.1016/S0140-6736(17)32714-9.

114. Petraco R, Escaned J, Sen S, et al. Classification performance of instantaneous wavefree ratio (iFR) and fractional flow reserve in a clinical population of intermediate coronary stenoses: results of the ADVISE registry. EuroIntervention. 2013;9(1):91-101. doi:10.4244/EIJV9I1A14.

115. Escaned J, Echavarria-Pinto M, Garcia-Garcia HM, et al. Prospective Assessment of the Diagnostic Accuracy of Instantaneous Wave-Free Ratio to Assess Coronary Stenosis Relevance: Results of ADVISE II International, Multicenter Study (ADenosine Vasodilator Independent Stenosis Evaluation II). JACC Cardiovasc Interv. 2015;8(6):824-833. doi:10.1016/j.jcin.2015.01.029.

116. Kang D-Y, Ahn J-M, Lee CH, et al. Deferred vs. performed revascularization for coronary stenosis with grey-zone fractional flow reserve values: data from the IRIS-FFR registry. Eur Heart J. 2018;360:213. doi:10.1093/eurheartj/ehy079.

117. Johnson NP, Jeremias A, Zimmermann FM, et al. Continuum of Vasodilator Stress From Rest to Contrast Medium to Adenosine Hyperemia for Fractional Flow Reserve Assessment. JACC Cardiovasc Interv. 2016;9(8):757-767. doi:10.1016/j.jcin.2015.12.273.

118. Shaw LJ, Berman DS, Maron DJ, et al. Optimal medical therapy with or without percutaneous coronary intervention to reduce ischemic burden: results from the Clinical Outcomes Utilizing Revascularization and Aggressive Drug Evaluation (COURAGE) trial nuclear substudy. Circulation. 2008;117(10):1283-1291. doi:10.1161/CIRCULATIONAHA.107.743963.

119. Johnson NP, Toth GG, Lai D, et al. Prognostic Value of Fractional Flow Reserve. Journal of the American College of Cardiology. 2014;64(16):1641-1654. doi:10.1016/j.jacc.2014.07.973.

120. Petraco R, van de Hoef TP, Nijjer S, et al. Baseline instantaneous wave-free ratio as a pressure-only estimation of underlying coronary flow reserve: results of the JUSTIFYCFR Study (Joined Coronary Pressure and Flow Analysis to Determine Diagnostic Characteristics of Basal and Hyperemic Indices of Functional Lesion Severity-Coronary Flow Reserve). Circulation: Cardiovascular Interventions. 2014;7(4):492-502. doi:10.1161/CIRCINTERVENTIONS.113.000926.

121. Hwang D, Jeon K-H, Lee JM, et al. Diagnostic Performance of Resting and Hyperemic Invasive Physiological Indices to Define Myocardial Ischemia: Validation With (13)NAmmonia Positron Emission Tomography. JACC Cardiovasc Interv. 2017;10(8):751760. doi:10.1016/j.jcin.2016.12.015.

122. Iskandrian AS, Heo J, Nguyen T, et al. Assessment of coronary artery disease using single-photon emission computed tomography with thallium-201 during adenosineinduced coronary hyperemia. The American Journal of Cardiology. 1991;67(15):11901194. doi:10.1016/0002-9149(91)90925-B. 
123. Greenwood JP, Maredia N, Younger JF, et al. Cardiovascular magnetic resonance and single-photon emission computed tomography for diagnosis of coronary heart disease (CE-MARC): a prospective trial. Lancet. 2012;379(9814):453-460. doi:10.1016/S01406736(11)61335-4.

124. Wilson RF, Wyche K, Christensen BV, Zimmer S, Laxson DD. Effects of adenosine on human coronary arterial circulation. Circulation. 1990;82(5):1595-1606. doi:10.1161/01.CIR.82.5.1595.

125. Dhalla AK, Wong M-Y, Wang W-Q, Biaggioni I, Belardinelli L. Tachycardia caused by A2A adenosine receptor agonists is mediated by direct sympathoexcitation in awake rats. Journal of Pharmacology and Experimental Therapeutics. 2006;316(2):695-702. doi:10.1124/jpet.105.095323.

126. Shryock JC, Belardinelli L. Adenosine and Adenosine Receptors in the Cardiovascular System: Biochemistry, Physiology, and Pharmacology. The American Journal of Cardiology. 1997;79(12):2-10. doi:10.1016/S0002-9149(97)00256-7.

127. Karamitsos TD, Ntusi NA, Francis JM, Holloway CJ, Myerson SG, Neubauer S. Feasibility and safety of high-dose adenosine perfusion cardiovascular magnetic resonance. Journal of Cardiovascular Magnetic Resonance. 2010;12(1):66. doi:10.1186/1532-429X-12-66.

128. Kidambi A, Sourbron S, Maredia N, et al. Factors associated with false-negative cardiovascular magnetic resonance perfusion studies: A Clinical evaluation of magnetic resonance imaging in coronary artery disease (CE-MARC) substudy. Journal of Magnetic Resonance Imaging. 2015;43(3):566-573. doi:10.1002/jmri.25032.

129. Siebes M. Intracoronary Doppler-Based Techniques for Stenosis Appraisal. Coronary stenosis imaging; 2010.

130. Pacold I, Hwang MH, Piao ZE, Scanlon PJ, Loeb HS. The mechanism and significance of ventricularization of intracoronary pressure during coronary angiography. Am Heart J. 1989;118(6):1160-1166.

131. Wilkinson IB, MacCallum H, Flint L, Cockcroft JR, Newby DE, Webb DJ. The influence of heart rate on augmentation index and central arterial pressure in humans. $J$ Physiol (Lond). 2004;525(1):263-270. doi:10.1111/j.1469-7793.2000.t01-1-00263.x.

132. Karamitsos TD, Ntusi NA, Francis JM, Holloway CJ, Myerson SG, Neubauer S. Feasibility and safety of high-dose adenosine perfusion cardiovascular magnetic resonance. Journal of Cardiovascular Magnetic Resonance. 2010;12(1):66. doi:10.1186/1532-429X-12-66.

133. Kramer CM, Barkhausen J, Flamm SD, Kim RJ, Nagel E. Standardized cardiovascular magnetic resonance imaging (CMR) protocols, society for cardiovascular magnetic resonance: board of trustees task force on standardized protocols. Journal of Cardiovascular Magnetic Resonance. 2008;10(1):35. doi:10.1186/1532-429X-10-35.

134. Kern MJ, Deligonul U, Tatineni S, Serota H, Aguirre F, Hilton TC. Intravenous adenosine: continuous infusion and low dose bolus administration for determination of coronary vasodilator reserve in patients with and without coronary artery disease. Journal of the American College of Cardiology. 1991;18(3):718-729. 
135. Alexopoulos D, Xanthopoulou I, Tsigkas G, et al. Effect of High ( $200 \mu \mathrm{g} / \mathrm{kg}$ per Minute) Adenosine Dose Infusion on Fractional Flow Reserve Variability. J Am Heart Assoc. 2016;5(11):e004323. doi:10.1161/JAHA.116.004323.

136. De Bruyne B, Pijls NHJ, Barbato E, et al. Intracoronary and intravenous adenosine 5'triphosphate, adenosine, papaverine, and contrast medium to assess fractional flow reserve in humans. Circulation. 2003;107(14):1877-1883. doi:10.1161/01.CIR.0000061950.24940.88.

137. Alberti C, Monopoli A, Casati C, et al. Mechanism and Pressor Relevance of the ShortTerm Cardiovascular and Renin Excitatory Actions of the Selective A2A-Adenosine Receptor Agonists. Journal of Cardiovascular Pharmacology. 1997;30(3):320-324. doi:10.1097/00005344-199709000-00008.

138. Biaggioni I, Killian TJ, Mosqueda-Garcia R, Robertson RM, Robertson D. Adenosine increases sympathetic nerve traffic in humans. Circulation. 1991;83(5):1668-1675. doi:10.1161/01.CIR.83.5.1668.

139. Headrick JP, Peart JN, Reichelt ME, Haseler LJ. Adenosine and its receptors in the heart: Regulation, retaliation and adaptation. Biochimica et Biophysica Acta (BBA) Biomembranes. 2011;1808(5):1413-1428. doi:10.1016/j.bbamem.2010.11.016.

140. Nayeem MA, Zeldin DC, Boegehold MA, et al. Modulation by salt intake of the vascular response mediated through adenosine $\mathrm{A}(2 \mathrm{~A})$ receptor: role of $\mathrm{CYP}$ epoxygenase and soluble epoxide hydrolase. Am J Physiol Regul Integr Comp Physiol. 2010;299(1):R325-R333. doi:10.1152/ajpregu.00823.2009.

141. Dixon AK, Gubitz AK, Sirinathsinghji DJS, Richardson PJ, Freeman TC. Tissue distribution of adenosine receptor mRNAs in the rat. British Journal of Pharmacology. 2012;118(6):1461-1468. doi:10.1111/j.1476-5381.1996.tb15561.x.

142. Gessi S, Merighi S, Varani K, Leung E, Mac Lennan S, Borea PA. The A3 adenosine receptor: An enigmatic player in cell biology. Pharmacology \& Therapeutics. 2008;117(1):123-140. doi:10.1016/j.pharmthera.2007.09.002.

143. Liu A, Wijesurendra RS, Ariga R, et al. Splenic T1-mapping: a novel quantitative method for assessing adenosine stress adequacy for cardiovascular magnetic resonance. Journal of Cardiovascular Magnetic Resonance. 2017;19(1):412. doi:10.1186/s12968016-0318-2.

144. Johnson SG, Peters S. Advances in pharmacologic stress agents: focus on regadenoson. Journal of Nuclear Medicine Technology. 2010;38(3):163-171. doi:10.2967/jnmt.109.065581. 